(NASA-CR-154288) AN EXPERIMENTAE

INVESTIGATION OF A THREF DIMENSTONAI WALL

JET Ph.B. Thesis (Virginia OHiv.) 214 p HC

A 10/MF A01 CSCI 20D

AN EXPERIMENTAL INVESTIGATION OF A

THREE DIMENSIONAI WALI JET

A Dissertation

Presented to

the Faculty of the School of Engineering and Applier srience University of Virginia

In Partial Fulfillment

of the Requirements for the Degree

Doctor of Philosophy (Aerospace Engineering)
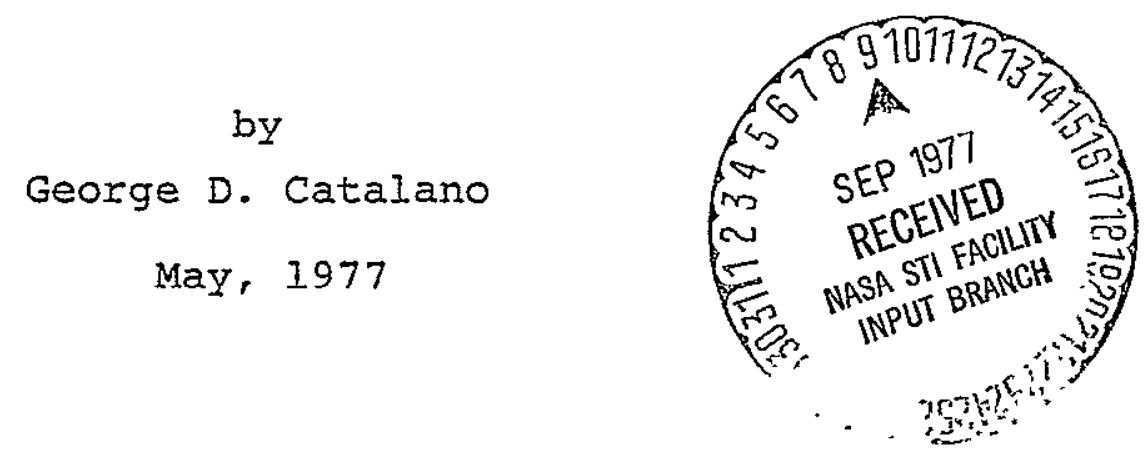


\section{APPROVAI SHEET}

This dissertation is submitted in partial fulfillment of the requirements for the degree of Doctor of Philosophy (Aerospace Engineering)

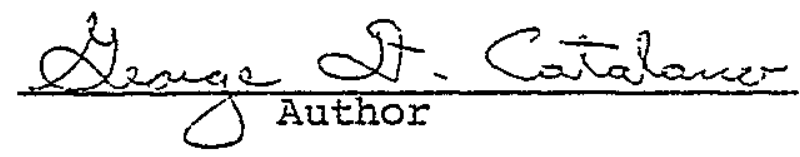

This dissertation has been read and approved by the Examining Committee:
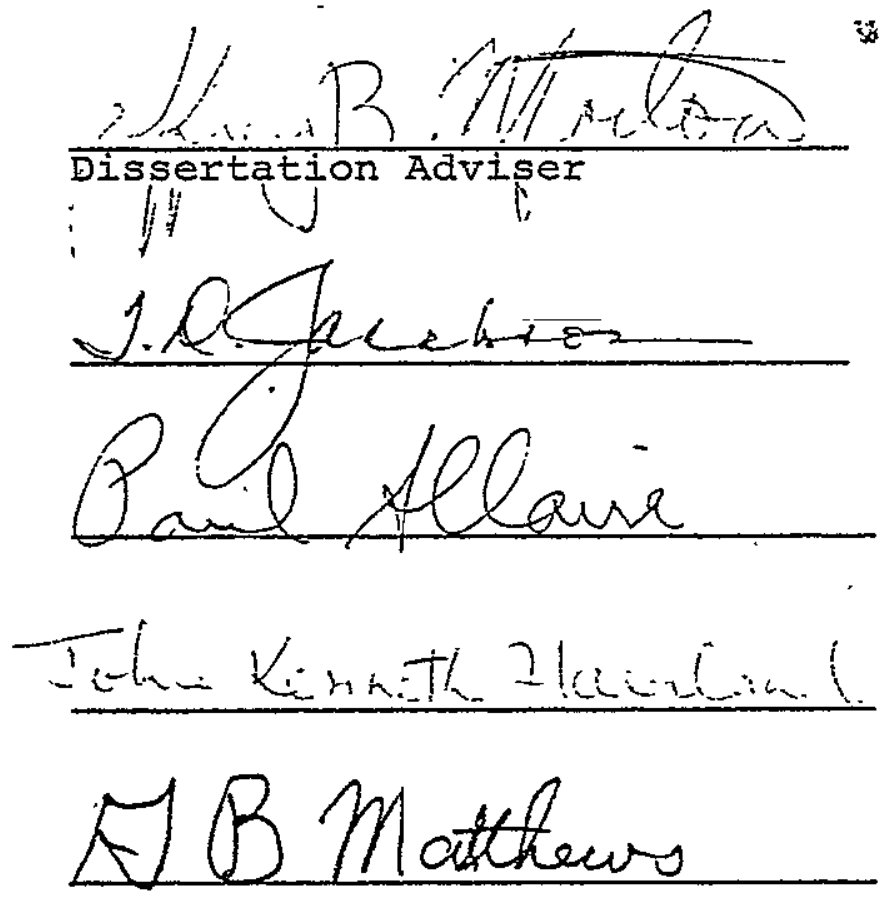

Accepted for the School of Engineering and Applied Science:

$$
\frac{4 . \sum . \text { Liblow }}{\text { Dedt, School of Engineering }}
$$




\section{ACKNOWLEDGEMENTS}

First, I would like to express my sincere thanks to my advisor, Dr. J. B. Morton. I consider myself most fortunate in, having been able to work for him over these past four years.

For the development of many of the electronic components used, I owe a great deal to Dr. R. R. Humphris. Whether it be questions concerning electronics or information in general, I often sought and always received help from him.

Iife in the E.S.S. Department could not have been as enjoyable were it not for the presence of my Iriend william w. Herling. The pressure-velocity measurements could not have been done without his help. To him, what can I say but a heart felt thank you. Thanks also go out to Tom Moul, G. Marcos Montes, and Yeu Chuan Hsia for their help in data reduction.

I would also like to express my appreciation for his continued interest and support for my career to my advisor at I.S.U., Dr. Robert W. Courter. His friendship means a great deal to me. Words really are inadequate to describe my appreciation to my parents. It is really hard to believe all that they have done for me over the years. 
For the concern and caring shown to me by Peg and Rose Anne, I shall forever be grateful.

Finally, to Shelley, her name in Scottish is so very appropriate to describe her influence on me. She is the "beautiful lake" that I go to for confidence, reassurance, and counsel.

Work supported by NASA Grant No. NGR47-005-219 and by NSF Grant No. Engr. 75-22488. 
TABLE OF CONTENTS

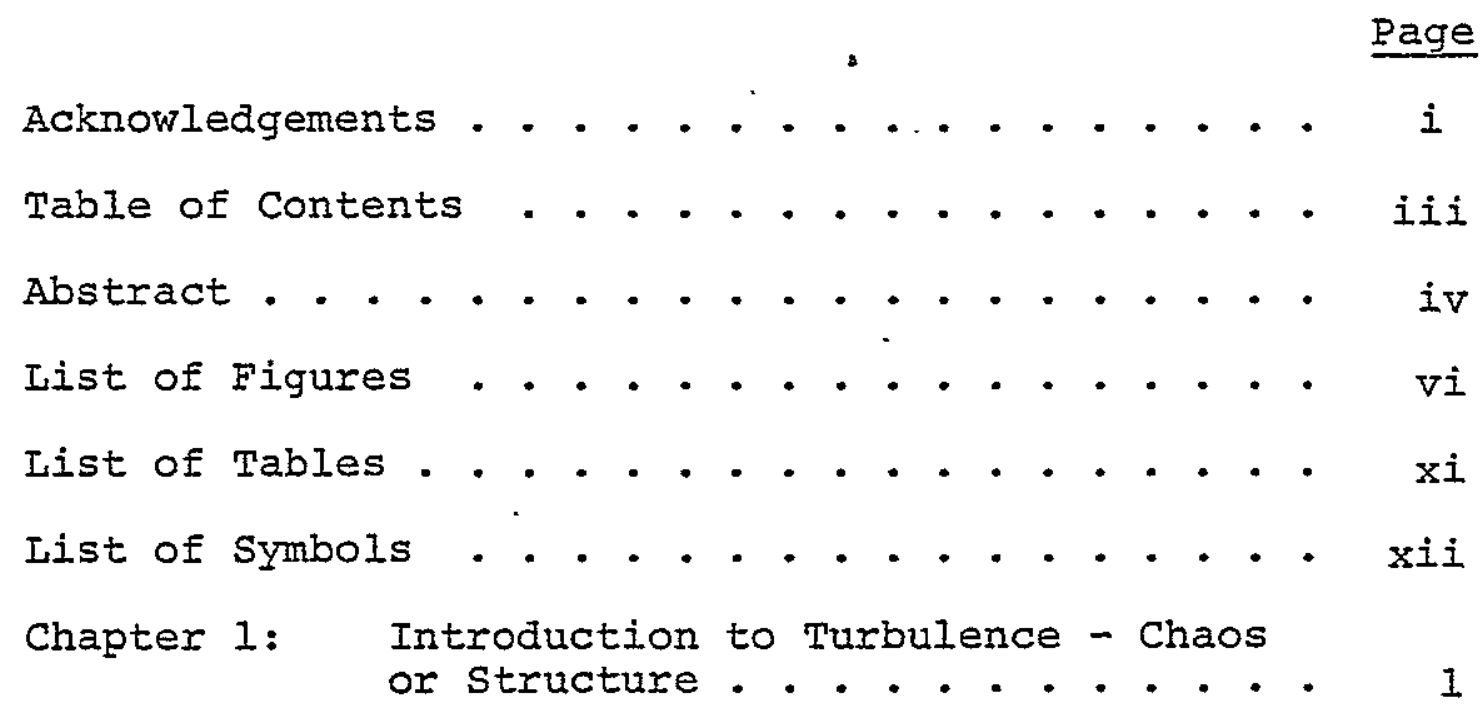

Chapter 2: Iaser Doppler Velocimetry . . . . 15

Chapter 3: Equipment and Procedures ... . . . 21

Chapter 4: Experimental Results and

Discussion . . . . . . . ... 43

Chapter 5: Summary . . . . . . . . . 156

Appendix A: Effects of Finite Sized Control

Volume and Doppler Quality

Discussion ......... . . 164

Appendix B: Particle Dynamics . . . . . . . 178

Appendix C: Phase Locked-Loop Processor . . . 182

Appendix D: Lateral Mean and Rms Velocity
Measurements . . . . . . . 188

References . . . . . . . . . . . . . 190 


\section{ABSTRACT}

One and two point statistical properties are measured in the flow fields of a coflowing turbulent jet. Two different confining surfaces (one flat, one with large curvature) are placed adjacent to the lip of the circular nozzle, and the resultant effects on the flow field are determined. The one point quantities measured include mean velocities, turbulent intensities, velocity and concentration autocorrelations and power spectral densities, and intermittencies. From the autocorrelation curves, the Taylor microscale and the integral length scale are calculated. Two point quantities measured include velocity and concentration space-time correlations and pressure velocity correlations. From the velocity space-time correlations, iso-correlation contours are constructed along with the Iines of maximum maximorum. These Iines allow a picture of the flow pattern to be. determined. The pressures monitored in the pressurevelocity correlations are measured both in the flow field and at the surface of the confining wall(s).

The velocity measurements are made with a laser Doppler velocimeter in conjunction with a phase locked-10op processor. The intermittency profiles are determined using a Iight scattering technique developed by shaughnessy and 
Morton. A $1 / 8$ inch condensor microphone is used as a pressure transducer.

The Reynolas number of the flow at the exit plane of the nozzle is 22,600 . Two ratios of the jet velocity at the exit plane to the speed of the outer tunnel are used in the investigation and they are $\lambda_{j}=5.1$ and $\lambda_{j}=10.88$. 
Morton. A $1 / 8$ inch condensor microphone is used as a pressure transducer.

The Reynolas number of the flow at the exit plane of the nozzle is 22,600 . Two ratios of the jet velocity at the exit plane to the speed of the outer tunnel are used in the investigation and they are: $\lambda_{j}=5.1$ and $\lambda_{j}=10.88$. 


\section{IIST OF FIGURES}

No.

Figure

$\underline{\text { Page }}$

I Schematic of a Wall Jet . . . . . . . .

2 Total Pressure Probe surveys of Coflowing

Jet, $\left(\lambda_{j}=5.1\right) \cdot . \cdot \cdot \cdot \cdot \cdot \cdot \cdot \cdot 24$

3 Flat Plate Assembly - Pressure Port

Locations (t) Shown (Half-scale Drawing),

and Pressure Port Schematic . . . . . . . 26

4 Curved Wall (Flap) Assembly - Pressure

Port Locations (t) Shown . . . . . . . 28

5 Vertical PIane Pressure Probe Surveys of

Jet/Flap, $\left(\lambda_{j}=5.1\right)$. . . . . . • . 30

6 (a) Jet/Flap and Jet/Plate setup . . . . . . 31

6(b) Schematic of Laboratory Facility . . . . . 31

7 (a) Two-color IDV Set-up . . . . . . . . . 33

7 (b) Jet and Plate Installed in Wind Tunnel

Test Section (Note outline of jet on

surface of the plate) . . . . . . . 33

7 (c) Dual Beam Receiver/Detector (Diode) . . . . 34

7 (d) Back Scattering Receiver/Detector (Photo-

multiplier tube) . . . . . . . . . . 34

8 Schematic of Electronics setup . . . . . 38

9 Longitudinal Mean Velocity Profiles,

$\mathrm{z} / 2 \mathrm{r}_{\mathrm{o}}=0.5,\left(\lambda_{j}=5.1\right) \cdot \cdot \cdot \cdot \cdot \cdot \cdot \cdot 45$

10 (a) Growth of Mixing Width, $\lambda_{j}=5.1$. • • • . 47

10 (b) Growth of Mixing Width, $\lambda_{j}=10.88$. • • 47

11 (a) Decay of Centerline Mean Velocity,

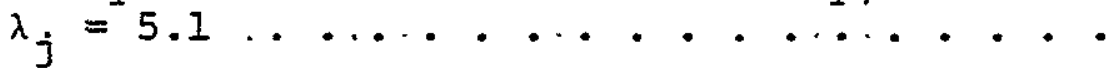

II(b) Decay of Centerline Mean Velocity,

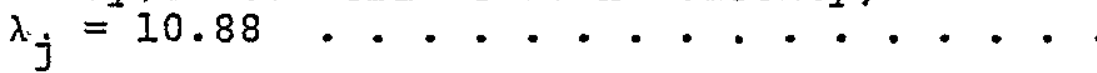


12 Development of Mean Velocity Profile at Jet Axis in Vertical, Plane for Flap . . . . . . . . . . . . . . . .

13 Iongitudinal and Vertical Mean Velocity Profiles for Flap . . . . . . . . . . 54

14 Vectorial Diagram of Flow over Flap . . 55

15 Vertical and Lateral Mean Velocity Profiles for Flap . . . . . . . . . . . . 57

16 Comparisons to Classical WaIl Jet Shape. .. 59

17 (a) Mean Velocity Profiles in Horizontal Planes Comparison to Similarity Curve . . 60

I7(b) Mean Velocity Profiles in Horizontal planes Comparison to Similarity Curves ... . ...

18 Iongitudinal Turbulent Intensities Profiles, $\lambda_{j}=5.1 . \cdot . \cdot . \cdot . \cdot . \cdot 65$

19 Iateral Turbulent Intensity Comparison . . 67

20 Development of Iateral Turbulent Intensity Profiles for Flow over Flap . . . . . 68

21 (a-b) Growth of Turbulent Intensity at CenterIine, $\lambda_{j}=5.1$ and $\lambda_{j}=10.88 \cdot \cdot \cdot \cdot \cdot \cdot \cdot 70$

22 Autocorrelations for Jet and Jet/Flap,

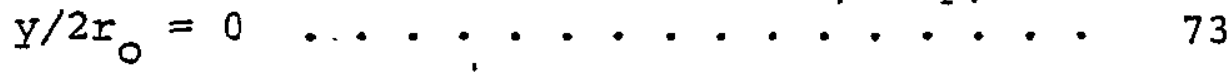

23 Autocorrelations for Jet and Jet/Flap, $y / 2 r_{0}=0.5 \cdot . \cdot \cdot \cdot \cdot \cdot \cdot \cdot \cdot \cdot \cdot 75$

24 Autocorrelations for Jet/Plate . . . . 77

25 Autocorrelations for Jet, $y / 2 r_{0}=0$. * 79

26 Autocorrelations for Jet, $y / 2 r_{0}=0.5$ • 80

27 .. Autocorrelations for Jet/Plate, $\mathrm{y} / 2 \mathrm{r}_{\mathrm{O}}=0$. . . . . . . . . . . . . . 
No.

Figure

$\underline{\text { Page }}$

28 Autocorrelations for Jet/Plate, $y / 2 r_{0}=0.5 \cdot \cdot \cdot \cdot \cdot \cdot \cdot \cdot \cdot \cdot \cdot \cdot \cdot \cdot \cdot \cdot$

29 Autocorrelations for Jet/Flap,

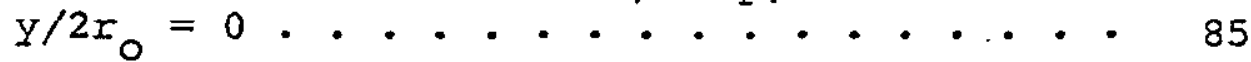

30 Autocorrelations for Jet/FIap, $\mathrm{y} / 2 \mathrm{r}_{\mathrm{O}}=0.5 \cdot \cdot \cdot \cdot \cdot \cdot \cdot \cdot \cdot \cdot \cdot \cdot \cdot \cdot \cdot$

31 (a) Development of Integral Scale for Jet,

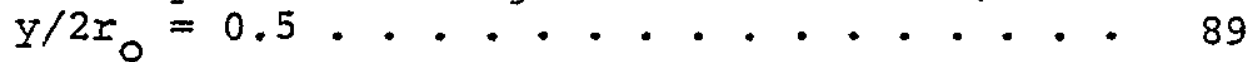

31 (b) Development of Integral scale for Jet,

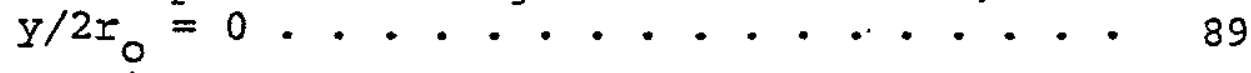

32 Development of Integral Scale for Jet/ Flap . . . . . . . . . . . . . . 92

33 (a) Development of Integral Scale for Jet/ Plate, $\lambda_{j}=10.88 \cdot . \cdot$. . . . • . 92

33 (b) Development of Integral Scale for Jet/ Plate, $\lambda_{j}=5.1 . . \cdot . \cdot . \cdot . \cdot . \cdot 93$

34 Power Spectral Density Comparison at $\mathrm{x} / 2 r_{0}=2 \cdot \cdot \cdot \cdot \cdot \cdot \cdot \cdot \cdot \cdot \cdot \cdot \cdot \cdot \cdot 99$

35 Power Spectral Density Comparison at $\mathrm{x} / 2 r_{0}=4 \cdot \cdot \cdot \cdot \cdot \cdot \cdot \cdot \cdot \cdot \cdot \cdot \cdot \cdot 101$

36 Downstream Development of Power Spectral Density for Jet, $\mathrm{y} / 2 \mathrm{r}_{\mathrm{o}}=0$. . . . . ' 103

37 Downstream Development of Power Spectral Density for Jet, $y / 2 x_{0}=0.5$. . . . 104

38 Downstream Development of Power Spectral Density for Jet/Plate, $y / 2 r_{0}=0$. . . . 105

39 Downstream Development of Power Spectral Density for Jet/Plate, $y / 2 r_{0}=0.5 . . .106$

40 Downstream Development of Power Spectral Density for Jet/FIap, $y / 2 r_{0}=0 . . \cdot . \cdot .108$

41 Downstream Development of Power Spectral Density for Jet/Flap, $y / 2 r_{0}=0.5 . . . .109$ 
42 Intermittency Profiles Comparison,

$\lambda_{j}=5.1 \cdot \cdot \cdot \cdot \cdot \cdot \cdot \cdot \cdot \cdot \cdot \cdot \cdot \cdot \cdot \cdot \cdot$

43 Concentration Auto and Cross Correlations, Jet, $\mathrm{x} / 2 \mathrm{r}_{\mathrm{o}}=4 \cdot \cdot \cdot \cdot \cdot \cdot \cdot \cdot \cdot \cdot \cdot \cdot \cdot \cdot 114$

44 Concentration Auto and Cross Correlations,

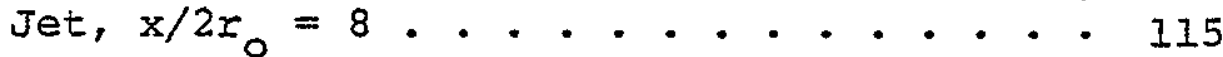

45 Concentration Auto and Cross Correlations, Jet/Plate, $x / 2 r_{0}=4$. . . . . • • • 117

46 Concentration Auto and Cross Correlations, Jet/Plate, $x / 2 r_{0}=8$. . . . . . . • 118

47 Concentration Auto and Cross Correlations, Jet/Flap, $\mathrm{x} / 2 \mathrm{r}_{0}=4$. . . . . . . . 120

48 Concentration Spectra Comparison,

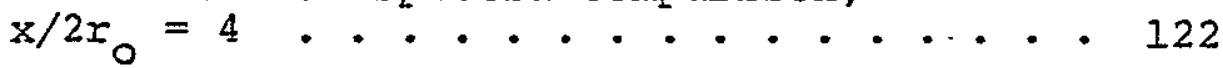

49 Concentration Spectra Comparison,

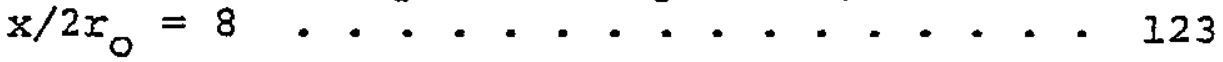

50 Isocorrelation Curves for Jet in $x-z$ Plane • • • • • • • • • • • • • 126

51 Isocorrelation Curves for Jet/Plate in x-z Plane . . . . . . . . . . . . . . 128

52 Isocorrelation Curves for Jet/Flap in.. $\mathrm{x}-\mathrm{z}$ Plane .............. $130^{\circ}$

53 Isocorrelation Curves for Jet in $\mathrm{y}-\mathrm{z}$ plane . . . . . . . . . . . . . . 133

54 Isocorrelation Curves for Jet/Plate in Y-z plane. . . . . . . . . . . . . . 134

55 Isocorrelation Curves for Jet/Flap in $y-z$ plane . . . . . . . . . . . 135

56 Wall Pressure-Velocity Coherence Plot for Plate, $\xi_{3} / 2 r_{0}=0.2 \cdot \cdot \cdot \cdot \cdot \cdot \cdot \cdot \cdot 144$

57 Wall Pressure-Velocity Coherence Plot for Plate, $\xi_{3} / 2 r_{0}=0.5$. . . . . . . . 145 
No.

58 Wall Pressure-Velocity Coherence plot for Flap, $\xi_{3} / 2 r_{0}=0.2 . . . \cdot \cdot \cdot \cdot \cdot \cdot$

59 Wall Pressure-Velocity Coherence for

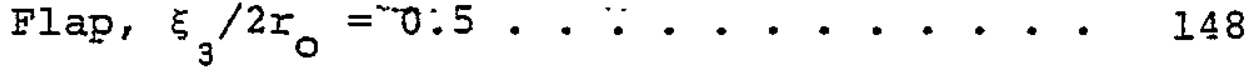

60 Lateral Displacement of Eressure Probe Effects on $\overline{\mathrm{Du}}$ Coefficient . . . . . . . .

61 Static Pressure-Velocity Coherence Plot for Jet, $\xi_{3} / 2 r_{0}=0 \quad$. . . . . . . .

62 Static Pressure-Velocity Coherence Plot for Jet at $x / 2 r_{0}=8$. . . . . . . . 152

63 Static Pressure-Velocity Coherence Plot for Jet/Plate at $\xi_{3} / 2 r_{0}=0$. . . . .

64 Static Pressure-Velocity Coherence Plot for Jet/Flap at $\xi_{3} / 2 r_{0}=0.0 \cdot . \cdot \cdot . \cdot 154$

Appendices - Figures

65 (a) Doppler Signals in Forward Scattering Modes . . . . . . . . . . . . . .

65 (b) Doppler Signal Versus Noise in Back Scattering Mode . . . . . . . . . 176

66 (a) Feedback Loop Model . . . . . . . . 183 66 (b) Iinear Model of Phase Locked-Ioop . . . . . 183 


\section{IIST OF TABLES}

No.

Table

Page

1

Comparison of the Taylor Microscale . . . 95

2 Convection Velocities in the Various

Flows . . . . . . . . . . . . . . 139

3 Evaluation of Effect of Control

Volume Size on Mean Square

Fluctuations . . . . . . . . . . . . 169

4 Comparison of Forward and Back

Scattering . . . . . . . . . . . . 173 


\section{IIST OF SYMBOLS}

\begin{tabular}{|c|c|}
\hline A & Cross-sectional Area of Control Volume \\
\hline B & Statistical Property \\
\hline$C_{D}$ & Drag Coefficient \\
\hline D & Diameter of particle \\
\hline e & Base for natural logarithm \\
\hline $\mathrm{E}$ & Voltage oútput \\
\hline$E(k)$ & Three dimensional energy spectrum function \\
\hline E & Frequency of fluctuation \\
\hline$f_{D}$ & Doppler shift in frequency of light \\
\hline$F_{1}$ & Similarity function in vertical plane \\
\hline $\mathrm{F}_{2}$ & Similarity function in horizontal plane \\
\hline$F(\tilde{F})$ & Power spectral density \\
\hline$F(s)$ & Current transfer function of the LPF \\
\hline$F_{11}, G_{22}, G_{1}$ & $\begin{array}{l}\text { Fourier Transform of autocorrelation functions } \\
\text { and cross correlation function }\end{array}$ \\
\hline$i_{d}(t)$ & $\begin{array}{l}\text { Low pass filtered phase detector's output } \\
\text { current }\end{array}$ \\
\hline$i_{e}(t)$ & Phase detector's output current \\
\hline I & Intermittency \\
\hline $\mathrm{k}$ & Wave number \\
\hline$\overline{\mathrm{k} s}$ & Wave vector of scattered light \\
\hline $\bar{k}_{L_{L}}$ & Wave vector of incident light \\
\hline $\mathrm{K}$ & $\begin{array}{l}\text { Constant relating mean and fluctuating } \\
\text { velocities }\end{array}$ \\
\hline$d^{\prime} K_{0}$ & Conversion gains of the $\mathrm{PD}$ and the $\mathrm{CCO}$ \\
\hline
\end{tabular}




\begin{tabular}{|c|c|}
\hline $\mathrm{K}_{1}, \mathrm{~K}_{2}, \mathrm{~K}_{3}$ & $\begin{array}{l}\text { Arbitrary constants } \\
\text { Stokes coefficient }\end{array}$ \\
\hline I & Longitudinal integral scale \\
\hline $\mathrm{n}$ & Index of refraction \\
\hline p & Static and Wall Pressure \\
\hline $\mathrm{P}$ & Velocity of particle in $\left(\overline{\mathrm{k}}_{\mathrm{S}}-\overline{\mathrm{k}}_{\mathrm{L}}\right)$ direction \\
\hline PSD & $\begin{array}{l}\text { Power spectral density on dB referenced to } \\
\text { dynamic pressure }\end{array}$ \\
\hline q & Particle Velocity component \\
\hline$Q$ & Velocity of particle in control volume \\
\hline$r$ & Particle velocity component \\
\hline$r_{0}$ & Radius of jet \\
\hline $\mathrm{R}$ & Autocorrelation coefficient (velocity) \\
\hline $\mathrm{R}_{\mathrm{C}}$ & Autocorrelation coefficient (concentration) \\
\hline $\mathrm{R}_{\mathrm{C}_{1} \mathrm{C}_{2}}$ & Cross correlation coefricient (concentration) \\
\hline Rụu' & $\begin{array}{l}\text { Two point correlation coefficient with } \\
\text { longitudinal separation }\end{array}$ \\
\hline $\begin{array}{l}\mathrm{R}_{1,1} \\
\text { Rpu }\end{array}$ & $\begin{array}{l}\text { Two point space-time correlation coefficient } \\
\text { Pressure-velocity correlation coefficient }\end{array}$ \\
\hline Iine, $s$ & Line of maximum maximorum \\
\hline S & Mean velocity gradient in control volume \\
\hline$t$ & Time \\
\hline$T$ & Time over which quantity is averaged \\
\hline$u, u^{-}, u^{*}$ & Longitudinal velocity fluctuations. \\
\hline$\underline{\underline{u}}$ & \\
\hline
\end{tabular}




\begin{tabular}{|c|c|}
\hline in & Measured turbulent velocity Eluctuations \\
\hline$u_{0}^{1}$ & $\begin{array}{l}\text { Turbulent fluctuations at center of control } \\
\text { volume }\end{array}$ \\
\hline U & Mean velocity in $x$ direction \\
\hline$U(A, t)$ & Instantaneous velocity in control volume \\
\hline Uam & Instantaneous measured velocity \\
\hline $\mathrm{UC}(t)$ & $\begin{array}{l}\text { Instantaneous velocity at center of control } \\
\text { volume }\end{array}$ \\
\hline Ucon & Convection velocity \\
\hline UES & Mean velocity of outer tunnel flow \\
\hline Ug & Mean velocity of gas \\
\hline Um & $\begin{array}{l}\text { Maximum mean velocity at a given downstream } \\
\text { location }\end{array}$ \\
\hline Uo & Mean velocity at exit plane of nozzle \\
\hline Up & Mean velocity of particle \\
\hline $\bar{U}_{1}$ & $\begin{array}{l}\text { Average value of mean velocity in case of } \\
\text { strong inhomogeniety. }\end{array}$ \\
\hline$\checkmark$ & Iateral velocity fluctuations \\
\hline $\mathrm{V}$ & Iateral mean velocity \\
\hline Vi & Amplitude of input signal \\
\hline Vo & Amplitude of CCo output signal \\
\hline $\mathrm{W}$ & $\begin{array}{l}\text { Control volume dimension in the direction of } \\
\text { mean velocity gradient }\end{array}$ \\
\hline$w_{i}$ & Frequency of input signal \\
\hline$\dot{w}_{0}$ & Frequency of CCO output signal \\
\hline$\underline{W}$ & Input and output frequencies of PII are the same \\
\hline W & Vertical mean velocity \\
\hline
\end{tabular}




\begin{tabular}{|c|c|}
\hline $\mathrm{x}$ & Longitudinal distance from exit plane \\
\hline$y$ & Lateral displacement from center of nozzle \\
\hline $\mathrm{Y}_{\mathrm{m}}$ & Velocity field half width in $x-y$ plane \\
\hline$z$ & Vertical displacement from lower lip of nozzle \\
\hline$x_{1 / 2}$ & Velocity field half width \\
\hline$\propto$ & Related to acceleration of particles \\
\hline$B$ & Angle between $\bar{k}_{S}, \bar{k}_{I}$ \\
\hline$\delta r_{12}^{2}$ & Coherence \\
\hline$n$ & $\begin{array}{l}\text { Lateral displacement from center of control } \\
\text { volume }\end{array}$ \\
\hline$\lambda_{j}$ & $\begin{array}{l}\text { Ratio of jet velocity to tunnel flow velocity } \\
\text { at exit plane }\end{array}$ \\
\hline$\lambda_{0}$ & Wavelength of laser beam \\
\hline$\lambda_{T}$ & Taylor Miscroscale \\
\hline$\mu$ & Viscosity of fluid \\
\hline$\xi_{1}$ & $\begin{array}{l}\text { Relative displacement between two "probes" in } \\
\text { x direction }\end{array}$ \\
\hline$\xi_{2}$ & $\begin{array}{l}\text { Relative displacement between two "probes" in } \\
y \text { direction }\end{array}$ \\
\hline$\xi_{3}$ & $\begin{array}{l}\text { Relative displacement between two "probes" in } \\
\text { z direction }\end{array}$ \\
\hline$\rho^{g}$ & Fluid density \\
\hline$\rho_{p}$ & Particle density \\
\hline$\sigma$ & Specific gravity \\
\hline$\tau$ & Delay time between two signals \\
\hline$\tau_{\mathrm{m}}$ & Optimum delay time between two signals \\
\hline$\Phi$ & Phase of $\mathrm{CCO}$ output signal \\
\hline
\end{tabular}




$\begin{array}{cl}{ }_{0} & \text { Phase angle of } 90 \text { degrees } \\ \Delta \phi & \text { Shift in phase angle } \\ (T . & \text { Time average of quantity }\end{array}$

xvi 


\section{Chapter 1}

INTRODUCTION TO TURBULENCE - CHAOS OR STRUCTURE.

\section{Section 1.0 Introduction}

Whether one observes the flows of water in rivers, lakes, and seas, examines the motions of gases in interstellar nebulae, or experiments with laboratory flows through pipes or nozzles, the conclusion is readily reached that turbulence is the most frequent state of fluidic behavior. Turbulent flows are described as flows in which velocity, pressure, and temperature are random functions of both time and space. (I) Generally, the image that is brought to focus in one's mind is of complete chaos and disorder. Special cases of organized motion existing along with the randomness and chaos of the more classically defined turbulence were known to exist such as, for example, vortex shedding from a circular cylinder up to a Reynolds number of $10^{7}$. But in these instances, the structures were regarded as being closely tied to their geometric origins and not characteristic of "well developed" turbulence. (2)

It has really only been in the past few years with the advent of improved statistical techniques and more careful observations that researchers have tried to search for understanding of turbulent flows that are not quite so 
chaotic as previously assumed. Conceptions in the past of the nature of turbulence were closely linked with the available experimental tools. In the forties, the hot wire technique for velocity measurements was sufficiently developed to test the assumptions of various phenomenological theories more directly. A complete rejection of these theories was the result. Advances in statistical techniques in the sixties resulted in some support being given to the turbulent viscosity concept as an engineering tool. With the continuing development in hot wire anemometry, experiments concentrated on studying the spectral distribution of the turbulent kinetic energy with a particular emphasis on the fine scale of turbulence. At the same time, various rate terms in the turbulent kinetic energy equation were investigated. (3)

Lately, many researches have focussed more attention on shear flow turbulence than the "simpler" but more academic problem of homogeneous turbulence. It was known that large structures are important in the development of turbulent shear flows. The earliest and most decisive attempts to define the form of large eddies were made by Townsend and his students. (4)-(5)

Kline and Runstaler ${ }^{(6)}$ and Brown and Roshko(7) reported observations that have had a significant effect on the direction of subsequent turbulent research. They used 
very basic optical methods and described that in turbulent flows of simple geometry, there is some order in the motion with an observable chain of events reoccurring randomly with a statistically definable mean.

As more and more evidence is obtained, the concept of spatially coherent structures has become increasingly reinforced. Also, the evidence has implied that the temporal behavior of these structures has a strong bearing on the development of the whole turbulent flow.

Section 1.1 The Mixing Layer

The simplest free shear flow that can be considered is the region between two parallel streams moving at different speeds. In the shear layer, the flow is usually in a turbulent state. The majority of the existing knowledge concerning shear layers either is conceived with the growth of small disturbances near the origin of the layer or with the fully turbulent section farther downstream. Michalke ${ }^{(8)}$ and stuart ${ }^{(9)}$ have reviewed the flow mechanisms at the start of the mixing layer. In the fully turbulent mixing layer, Wyganski and Fiedler ${ }^{(10)}$ divided their measurements into three categories: (1) conventional average measurements (2) time average measurements in the turbulent and non-turbulent zones and (3) ensemble average measurements conditioned to a specific location of the 
interface. They divided the mixing region into two parts, one on the high velocity side and one on the low velocity side. The interfaces on each side of the mixing layer moved independently of each other. Finally, the turbulence, which is not subjected to a constant mean shear (i.e. the gradient oscillates depending on the location of the interface) is not homogeneous and in general nonisotropic. Roshko et aI (11)-(12) looked at the effect of varying parameters of the mixing layer such as the velocity ratio, the density ratio, and Mach numbers. Shadow pictures of the flow revealed the presence of well defined large structures which have the appearance of breaking waves. It was also found that the mean properties of the flow were controlled by the large coherent structures and independent of the small scale turbulence.

The significant gap between the results of the work pertaining to the linear instability of shear layers and the descriptions of turbulent mixing layers was the focus of Winant and Browand. (13) Flow visualization was used along with hot film measurements of conventional mean properties and statistical measurements aimed at defining ensemble-averaged properties of the structures observed in the visual studies. Unstable waves were found to. grow, and the fluid was observed to roll up into discrete two dimensional vortical structures. These turbulent vortices' 
interacted by rolling around each other, and a single vortex was formed. This pairing process was observed to occur repeatedly, and it controlled the growth of the mixing layer.

A theoretical study of the rolling up process to which winant et al referred was presented by Moore. (14) For the first time, the chaotic motion which was a feature of earlier studies was eliminated and the details of the outer portion of the spiral were determined. This was accomplished by using a point-vortex representation to numerically study the evaluation of an initially plane vortex sheet. The rate of rolling up was also calculated.

Section 1.2 Structured Jet Turbulence

As the initial development of two dimensional and circular jets consists of two annular mixing layers forming around the central potential core, one expects these layers to behave in a similar manner to that described in the previous section.

The main physical features of a subsonic jet are described by Davies. (15) At the jetorifice there exists a thin, cylindrical vortex sheet which rapidly develops into a layer of highly sheared strong turbulence. This settles down to an annulus of strongly sheared, self-preserving, intense turbulence surrounding a core of potential flow 
after approximately one nozzle diameter. The statistical and geometric characteristics of the flow remain similar for another four or five diameters. A second adjustment region follows where the potential core has vanished and the mean shear decreases rapidly.

Crow and Champagne, (16) in their investigation of the noise producing region of a jet, studied the evolution of orderly flow with increasing Reynolds number. The instability of the jet changes from a sinusoidal surface ripple to a helix and then to a train of axisymmetric waves as the Reynolds number is increased from 100 to 1000 . At a Reynolds number equal to 10,000 , two axisymmetric structures which can be detected are surface ripples on the jet column and a train of large scale vortex puffs. Crow and Champagne. found that when a periodic surging of a given amptitude and frequency was imposed on the flow at the jet exit which caused the formation of a Iundamental wave, the preferred mode with a strouhal number of .3 was the most dispersive wave on the jet column, and least capable of generating a harmonic.

A. J. Yule ${ }^{(17)}$ described investigation of coherent motions in the first ten diameters of round jets. The turbulent flow was dominated by the interacting and coalescing of ring vortices. The large organized structures in the turbulent region of the flowing fluid differ funda- 
mentally from the laminar ring vortices at the exit plane. These structures possessed a strong and well ordered three dimensional nature.

Experimental and analytical work aimed at understanding the three dimensional interaction of discrete vortex rings and their stability characteristics have been présented by Kambe and Takeo ${ }^{(18)}$ and Oshima. (19) The coalescense process and the observed and predicted growth of vortex core waves had basic similarities with occurrences in continuously running round jets.

The structure of jets which are subjected to a periodic forcing has also been investigated by Girard, et. al. (20) and Binder. (21) Measurements indicated appreciable effects for forcing Strouhal numbers greater than 0.04 . The two most important effects were a higher initial spreading rate and an associated periodic velocity component which decayed downstream.

For further description of the information gathered concerning the flow of turbulent jets, please refer to Catalano, Morton and Humphris. (22)

Section 1.3 "Wall Jets

While more and more attention is being focussed on the search for structure in turbulent jets and mixing layers, the flow fields of three dimensional turbulent wall 
jets are virtually being ignored. Before progressing further, a definition of the term wall jet should be discussed. The presence of both a solid boundary and a free boundary of the same fluid as the jet leads to the term wall-jet which seems to have first been described by Glawert. (23)

As in a free or coflowing jet problem, in a wall jet there exist two main zones of the flow: (I) An initial zone of flow established immediately beyond the jet exit and (2) a zone of established flow (Figure 1). The initial zone can be further subdivided into the potential core where the turbulence level is still relatively low, the developing boundary layer on the surface of the wall and the mixing or transition region which has been theorized to have a similar flow mechanism to that of a free jet.

The zone of established flow can be further subdivided into a region of free mixing where the transport processes are again similar to a free jet and the region near the wall termed the inner layer. The inner layer cạn be considered a boundary layer growing under a turbulent, decelerating, super-stream. The upper edge of the boundary layer is the vertical location of maximum mean velocity at any downstream position. 


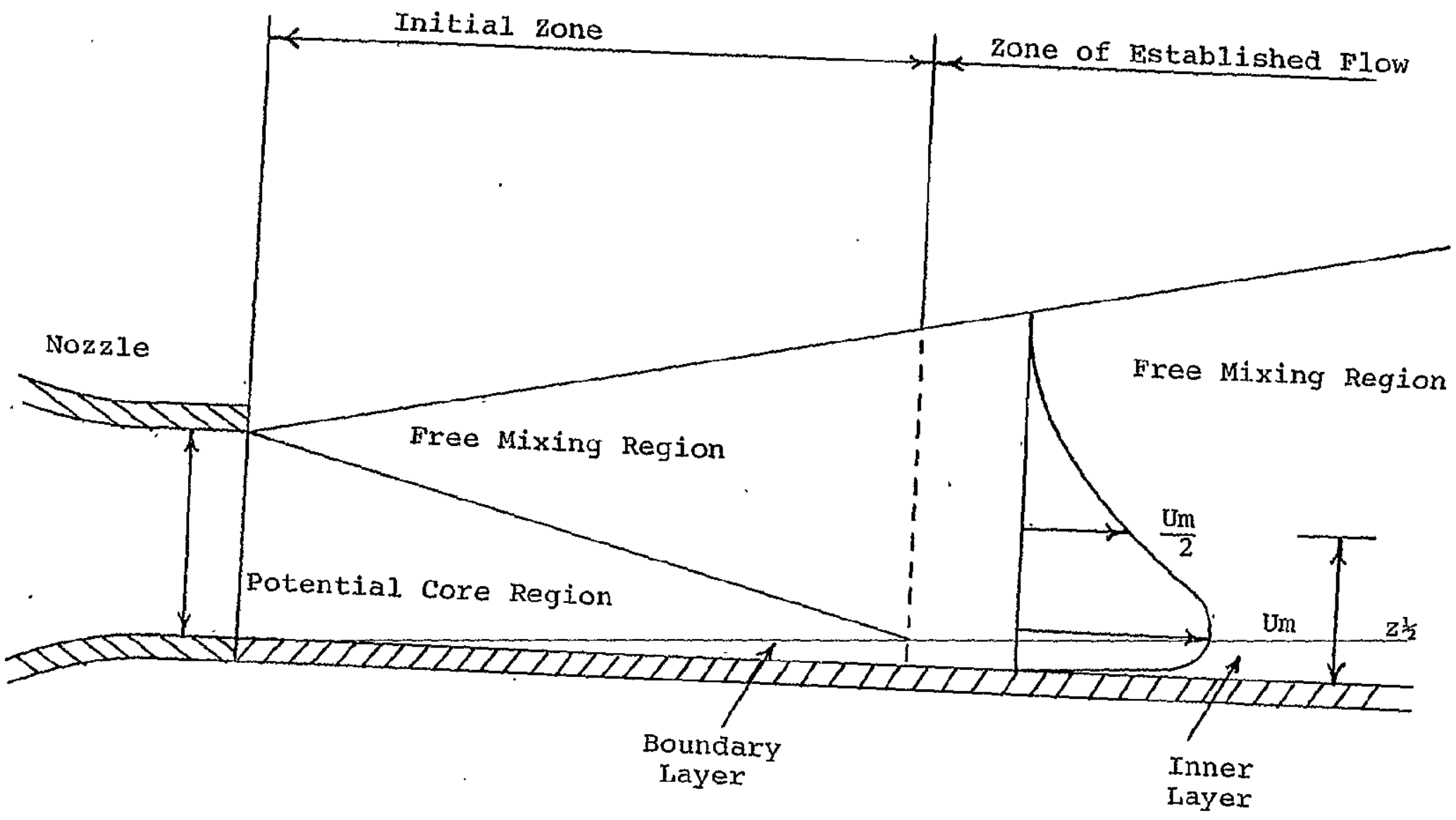

Figure 1 schematic of a Wall Jet 
The two dimensional wall jet was first investigated by Forthman ${ }^{(24)}$ in 1936. Glauert, (23) Schwarz and Cosart, (25)
Sigalla,
and Myer et. al.
(27) added theoretical

background and analysis to the problem. It was found that the mean velocity profiles, excluding the viscous sublayer near the wall, were similar. Mura Hari ${ }^{(28)}$ proposed analytical methods for predicting the flow development region and the bed shear distribution in a wall jet. Dvorak ${ }^{(29)}$ determined an analytical method for turbulent boundary layers and wall jets in which the effects of: large longitudinal surface curvature and the associated normal pressure gradients were included for the first time. The Reynolds stress terms in the equations of motion were approximated using an eddy viscosity approach based on the concept of intermittency. Blackwelder and Rovasznay (30) obtained space time correlations with large streamwise separation in a turbulent boundary layer with a zeropressure gradient. The use of conditional averaging techniques led to an average picture of the velocities in the interfacial bulges.

The three dimensional turbulent wall jet was investigated by Rajaratnam and Pani. (31) Experimental observations on the velocity and length scales, and wall shear stress for wall jets issuing from circular, square, trịangular, and elliptic nozzles were presented. It was 
found that the length scale in the transverse direction grew about five times as fast as the length scale in the vertical direction. Narain ${ }^{(32)}$ analytically investigated three dimensional flows and found that the maximum velocity shows three regions of decay. The potential core region is followed by a region where the velocity decay is dependent on the shape and aspect ratio of the orifice. Following this region is a radial wall jet type maximum velocity decay region. Hill (33) looked at the effect of confining walls on a coflowing turbulent jet. It was shown that before the jet spreads to the wall, both the mean velocity and turbulent shear stress are selfpreserving. Afterwards, only the shear stress is subject to this condition.

From the above discussion, it is clear that the recent changes in thinking concerning the structure of turbulence have not been applied to any significant degree in the study of wall jets. In fact, very little information has been gathered concerning the turbulence velocity field of a 3-dimensional wall jet. Also, even in: the measurement of mean velocity components nature of the flow was often times inferred analytically rather than actually measured.

In view of the new approach taken in the measurement of turbulent jets and mixing layers, it is the purpose of this investigation to determine the effects of. wall surfaces 
on the flow field of an axisymmetric jet in a coflowing air stream. Of primary interest in this investigation is the comparison of both one and two point statistical properties measured at the same locations. in the flow fields relative to the exit plane of the nozzle, with and without the wall structures present.

In order to best discern the effects of the wall structures, the velocity and concentration statistical properties of the unconfined turbulent jet must be well documented. This documentation was the subject of the author's Master's thesis, (34) The investigation was Iimited to one Reynolds number ( $R e=22,600)$. The statistical properties mapped included mean'velocities, turbulent intensities, intermittencies, and autocorrelations and power spectra for both the fluctuating velocity and concentration fields. The velocity measurements were taken with a Iaser Doppler velocimeter and the results compared to hot wire anemometer and pressure probe data where available. Mean velocity profiles as well as the decay of the centerline mean velocity were compared to the results of Iawrence. (35) Though there was an order of magnitude difference in the Reynolds numbers between the two investigations, the agreement was quite good. Axial and radial distributions of turbulent intensities were compared to those measured by Davies. ${ }^{(15)}$ and Lawrence. (35) Agreement 
again was quite reasonable. The conditionally sampled nature of the measurements led to the observance of somewhat higher turbulent intensities than was previously reported in the outer regions of the flow. The intermittency factor profiles were compared to data presented by Sarni, Carmody, and Rouse. ${ }^{(36)}$ The agreement was quite good. A rise in the intermittency in the region adjacent to the lip of the nozzle indicated the presence of a vortex ring. at the exit plane. The appearance of peaks in several of the velocity power spectra was in agreement with Ko and Davies ${ }^{(37)}$ and Bradshaw. (38) The spectrum shape was found to be completely aifferent in the potential core region as compared to the mixing region. In the potential core, the "bump" or peak in the power spectra were explained by Davies ${ }^{(15)}$ and Bradshaw et al. ${ }^{(38)}$ as being caused by the passing of vortices downstream. In the mixing region, the high background turbulence level masked out the relatively small velocity fluctuations due to the vortex street. Whenever possible, comparisons are made with data presented in the literature. However, as stated previously, very little information concerning three-dimensional wall jets was found to exist:

One application of the research would be in the area of short take-off and landing aircraft technology. Presently, one design under consideration is termed Upper 
Surface Blowing. The basic tenet of this approach is that the exhaust gases of the jet engine are turned by the presence of the aircraft wing's upper surface and any trailing edge flap. It has been found that a significant increase in lifting capability is realized; but the buffeting that the structure must endure is quite severe. Hence, there exists a need for more information for a configuration that can be classified a three dimensional turbulent wall jet. 
Chapter 2

\section{LASER DOPPIER - VELOCIMETRY}

\section{Section 2.0 IDV Background}

Since the first published account of the use. of the Laser Doppler Velocimeter (IDV) appeared in 1964, much effort has been devoted to the IDV's development. The fact that classical probing techniques such as pitot probes, temperature probes, or hot wires suffer from problems including frequency response, probe interference, high temperature environments, low gas densities and poor spatial resolution has made the IDV seem very promising in its use as a diagnostic tool. Because of the many details involved in the IDV as a system, the reader is referred to the proceedings of several IDV symposia $(40)-(4 I)$ as a beginning step. This section of the paper will try to incorporate some of the more recent published developments and include some of the progress of ongoing work. Much attention has been given to the analysis of the reliability of LDV measurements. Kried ${ }^{(42)}$ developed an approximate technique for an estimation of errors in velocity measurements due to flow variation within the finite dimensions of the scattering volume. The technique is applied to both frequency tracking and individual realization IDV applications. In addition, the analysis 
allows estimation of errors incurred when velocity measurements are made at locations sufficiently near the wall that part of the scattering volume is truncated by the wal1. A similar type of investigation was completed by Karpuk and Tiederman ${ }^{(43)}$. They found that velocity variance in the data originates from three sources: velocity fluctuations caused by turbulence at. the center of the probe volume; spatial variations in the mean velocity across the probe volume; and spatial variations in velocity fluctuations across the probe volume. Often times in the Iiterature, the term "ambiguity noise" is used to describe the errors resultant from a finite sized control volume. New applications for Laser Doppler Velocimetry are constantly appearing. Baker, Hutchinsen, and Whitelaw $(44)$ used an IDV system to measure the instantaneous velocity within flames. Continued development in this area might allow the IDV technique to be used in the analysis of flow in the cylinder of an internal combustion engine. Wisler and Mossey ${ }^{(45)}$ developed a system capable of investigating the flow field within the rotating blade row of a low speed axial compressor. The compactness and flexibility of the IDV technique was demonstrated by Muñoz, Mocker, and Koehler. (46) Munoz, et. al. developed $\mathrm{CO}_{2}$ laser Doppler detection system which can be used to measure remotely the true airspeed of an aircraft. Hughes and 
Pike ${ }^{(47)}$ considered several types of Laser Doppler Velocimetry which are suitable in the remote measurement of wind velocity. The most significant conclusion reached was that $\mathrm{CO}_{2}$ laser systems have considerably greater sensitivity than visible laser systems at ranges greater than a few meters. Design considerations for a 3-D IDV system for studying gravity waves in shallow water were reported by Hallermeier. (48) The laser velocimeter system used three frequency-modulated light beams and one detector for measurement of the instantaneous velocity vector in reversing flow.

Measurements in free and confined coaxial jets with recirculation were made by. Owen (49). Méasured turbulent kinetic energy and shear stress distributions indicated that nonequilibrium effects are dominant in the initial mixing regions and that higher order velocity moment turbulence models will probably be required to compute these flows. Velocity measurements of a slightly different sort were proposed and the results reported by wang, Clifford, and ochs. (50) Wind and refractive-turbulence. were sensed using crossed laser beams. The technique interprets the naturally occurring atmospheric modulation of the beam intensity (scintillation) to extract the average transverse speed and direction of air motion between source and receiver. 
Recently, the LDV has been applied to a variety of flows in both the transonic and supersonic regimes. These flows include jets, boundary layers, flow field around bodies, wakes, and cascades. Pfeiffer, vom steim, and Koch $^{(51)}$ have reported measurements of mean velocities of a free jet issuing from a shock tube where the run time was of the order of one millisecond. The results indicated that the LDV can be used to measure accurately. transient phenomena in high speed flows. Yanta and Iee ${ }^{(52)}$ and Rose and Johnson ${ }^{(53)}$ have made boundary layer measurements at a nominal Mach number of three with no pressure gradients. The final application to be discussed here was reported by Flack and Thompson. (54) In the report, measurements of velocities in the free stream (inviscid) part of a transonic flow are made. The effect of parameters such as signal-tomoise ratios, particle response, seeding rates, etc. were then documented.

Section 2.1 Laser Doppler Velocimetry - Theory

The general theory underlying the IDV technique is that the flow velocity at a point is determined by measuring the Doppler shift of monochromatic laser light scattered from small particles in the flow. To increase the amount of light scattered, the flow is often seeded by injection of particles of suitable size and density 
which are capable of following the fluctuations in the velocity field.

For the general scattering geometry, the Doppler shift is given by $f_{D}=\left(\bar{k}_{S}-\bar{k}_{L}\right) \cdot \bar{Q}$ where $\vec{k}_{S}$ and $\vec{k}_{\dot{L}}$ are the wave vectors of the incident and scattered radiation and $\bar{Q}=p \bar{i}+q \bar{j}+r \bar{m}$ represents the velocity $\bar{Q}$ in terms of components along and perpendicular to the direction of the mean flow, $i$. For the special case when the vector $\left(\overline{\mathrm{k}}_{S}-\overline{\mathrm{k}}_{\mathrm{L}}\right)$ is parallel to the $x$-direction then $E_{D}=(2 P / \lambda 0) \sin (B / 2)$ where $P$ is the angle between $\bar{k}_{S}$ and $\bar{k}_{L}$ and $\lambda_{0}$ is the wavelength of the monochromatic laser beam.

The question for each application of the IDV is to determine what parameters affect its reliability and to determine the result of the biasing of the flow field information when it is found to be important. The biasing, which is the error in the measurements made by the IDV technique, can be related to the basic parameters of the IDV setup and the properties of the velocity field, and the actual dynamics of the particles used to seed the flow.

In Appendix A, the effects of the finite sized laser control volume ${ }^{(55)}$ on the measurement of mean and fluctuating velocities are addressed. It is found that the surmation over a finite volume yields a good estimate of 
the mean velocity at the center of the probe under certain assumptions. The fluctuating velocities are seen to be influenced by any mean velocity and mean square fluctautions across the probe volume. The characteristics of the signal processing unit are also discussed as well as the quality of the Doppler signals detected.

In Appendix B, the question of how closely the dioctyl phthalate, DOP, particles follow the actual flow field is discussed. (56) The particle lag of the DOP particles in the nozzle is found to be negligible. Secondly, the particles act as a low pass filter with the $3 \mathrm{~dB}$ point of the rms respionse occuring at approximately $4 \times 10^{5} \mathrm{~Hz}$. 


\section{Chapter 3}

EQUIPMENT AND PROCEDURES

\section{Section 3.0 Flow System}

A jet whose compressed air is marked with dioctyl. phthalate (DOP) is mounted inside the test section of a Low turbulence level subsonic wind tunnel. The nozzle with a contraction ratio of 14 to 1 over a length of 15.9 $\mathrm{cm}$ has a Reynolds number of 22,600 and an exit plane diameter of $2.14 \mathrm{~cm}$. The entire jet assembly consists of a diffusion section, a flow straightening section which includes two Eine mesh screens and plastic coated soda straws, and the nozzle itself.

With the mounting of the assembly inside the wind tunnel, the capability exists for varying one of the more important parameters which characterize the flow field the ratio of the exit plane velocity of the jet to the uniform, parallel, constant outer flow velocity, $\lambda_{j} \cdot$ For the majority of the results reported in this investigation, the secondary flow in the tunnel was kept at $3.20 \mathrm{~m} / \mathrm{sec}$. This results in a value of 5.1 for $\lambda_{j}$. To ascertain the effects of changing the speed of the outer flow, a second larger value of $\lambda_{j}$ was chosen (i.e. $\lambda_{j}=10.88$ ). It should be pointed out that the speed of the tunnel flow was constantly monitored by use of a pressure probe and an 
electronic manometer (Barocel Type 1012).

The 1 meter long test section of the wind tunnel has the dimensions of $215 \mathrm{~mm}$ by $305 \mathrm{~mm}$. The walls of the test section are plexiglass with optical quality flat glass windows installed for better LDV performance. The floor of the test section was machined at several 10cations which enables the jet assembly to be positioned anywhere in the range from approximately zero to ten diameters upstream from the fixed $x$ location of the vertical laser "probe".

As was mentioned previously, the compressed air is marked with dioctyl phthalate (DOP). The DOP in conjunction with a Royco Model 258 Smoke Generator produces a smoke with a mean particle size of 0.3 microns. The output of the smoke generator is mixed with clean air from the Iaboratory's compressed air lines.

The wind tunnel, electronic equipment, and optical components, with the exception of the laser, are all placed inside a Iindgren double walled copper screen room. The copper screening filters out radio frequencies which are in the range monitored by the IDV equipment. Since the experimental design upstream of the jet is an important parameter, several methods were used in determining the actual conditions. First, smoke (DOP) 
was injected into the outer tunnel flow and the region. near the contraction section of the jet assembly was closely monitored. No evidence of separation of the outer flow from the jet was observed. Secondly, pitot-static tube profiles were taken for several $x / 2 r_{0}$ locations. Two such profiles are shown in Figure 2 for $\lambda_{j}=5.1$. At $x / 2 r_{j}=0$, the mean velocity is zero at the lip of the nozzle and then reaches the free stream value at approximately 1.5 diameters radially out from the lip. At $x / 2 r_{0}=I$, the velocity defect has decreased so that the smallest fluid velocity is about one half the value of the free stream velocity. This trend continues until at $x / 2 x_{0}=6$, virtually no defect is apparent.

Finally, a pressure probe. connected to $1 / 8$ inch condenser type microphone was traversed along the outside of the contraction section of the jet assembly. By the sound produced, it is easy to distinguish between laminar and turbulent flow. Once again no evidence of the separation of the tunnel flow from the jet assembly was found.

Section 3.1 Wall Surfaces

Two different types of confining surfaces are used in this investigation. The leading edge of either of the wall surfaces is placed adjacent to the lip of the nozzle. 

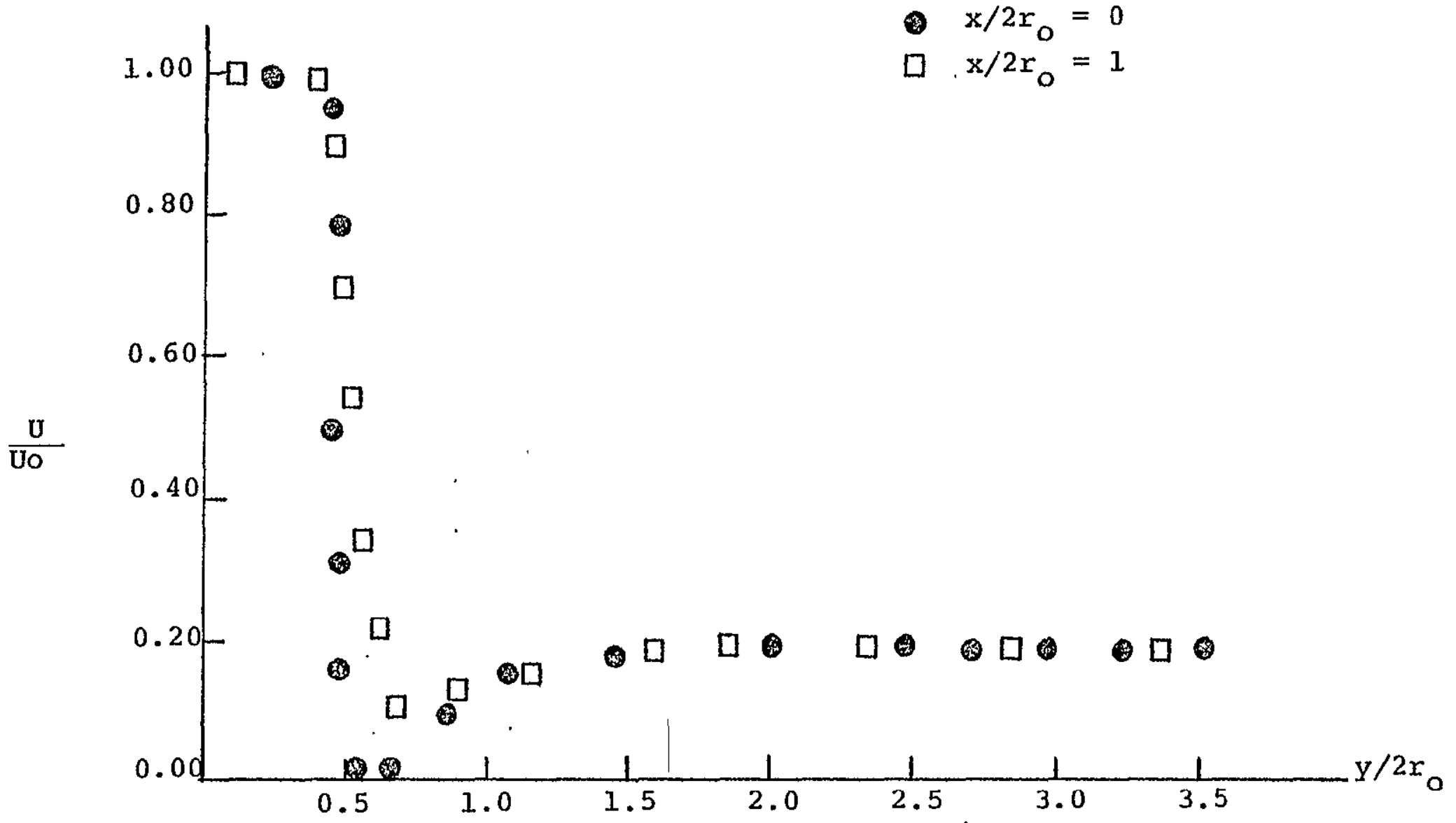

Figure 2 Total Pressure Probe Surveys of Coflowing Jet

$$
\left(\lambda_{j}=5.1\right)
$$


Care is used in the positioning of the walls so that a smooth transition is secured between the lower lip and the upper edge of the confining surface. The positioning is indicated in Figure 1. The first type of surface is a flat aluminum plate (henceforth denoted "plate") $30.48 \mathrm{~cm}$ long, $30.48 \mathrm{~cm}$ wide, and $.635 \mathrm{~cm}$ thick. Figure 3 gives a top and side view of the plate, and supports. The leading edge is contoured so that it disturbs the outer tunnel flow as little as possible. The entire assembly fits snugly into the wind tunnel test section. In order to use a forward scattering mode of LDV operation when the plate is placed in the test section, a portion of the aluminum has been milled out and replaced by an optical quality glass flat. This allows the scattered light to be detected below the surface of the plate. Actually, two flat plates were constructed. Since surface pressure-velocity correlations were sought in the investigation, pressure ports had to be mounted in the confining walls. The second flat plate was necessarily fabricated entirely out of aluminum with the pressure ports mounted flush with the supper surface. Figure 3 also presents a schematic view of the pressure ports themselves as well as the pattern of their location in the plate. 


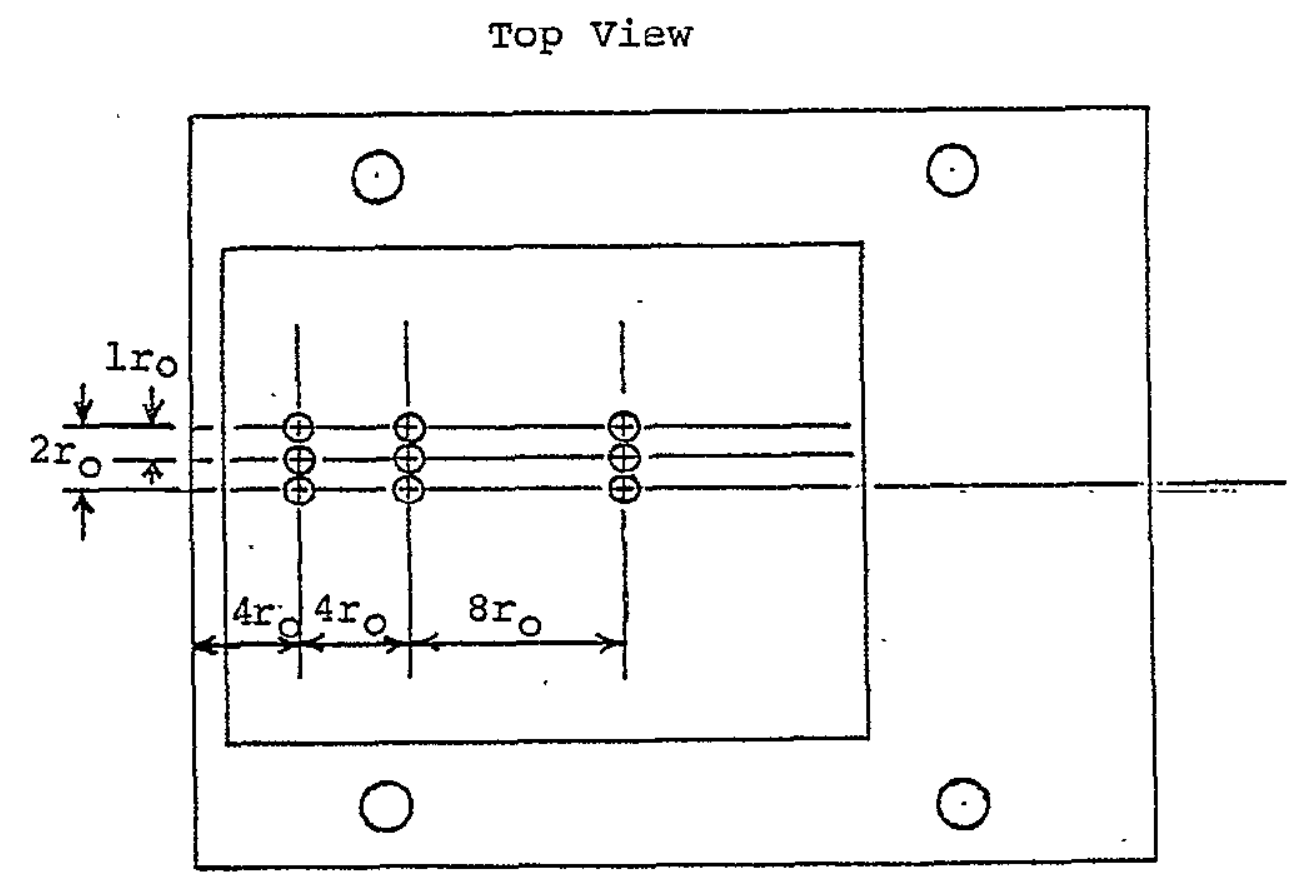

Side View

Pressure $.10 \mathrm{~cm}$ ID
Port

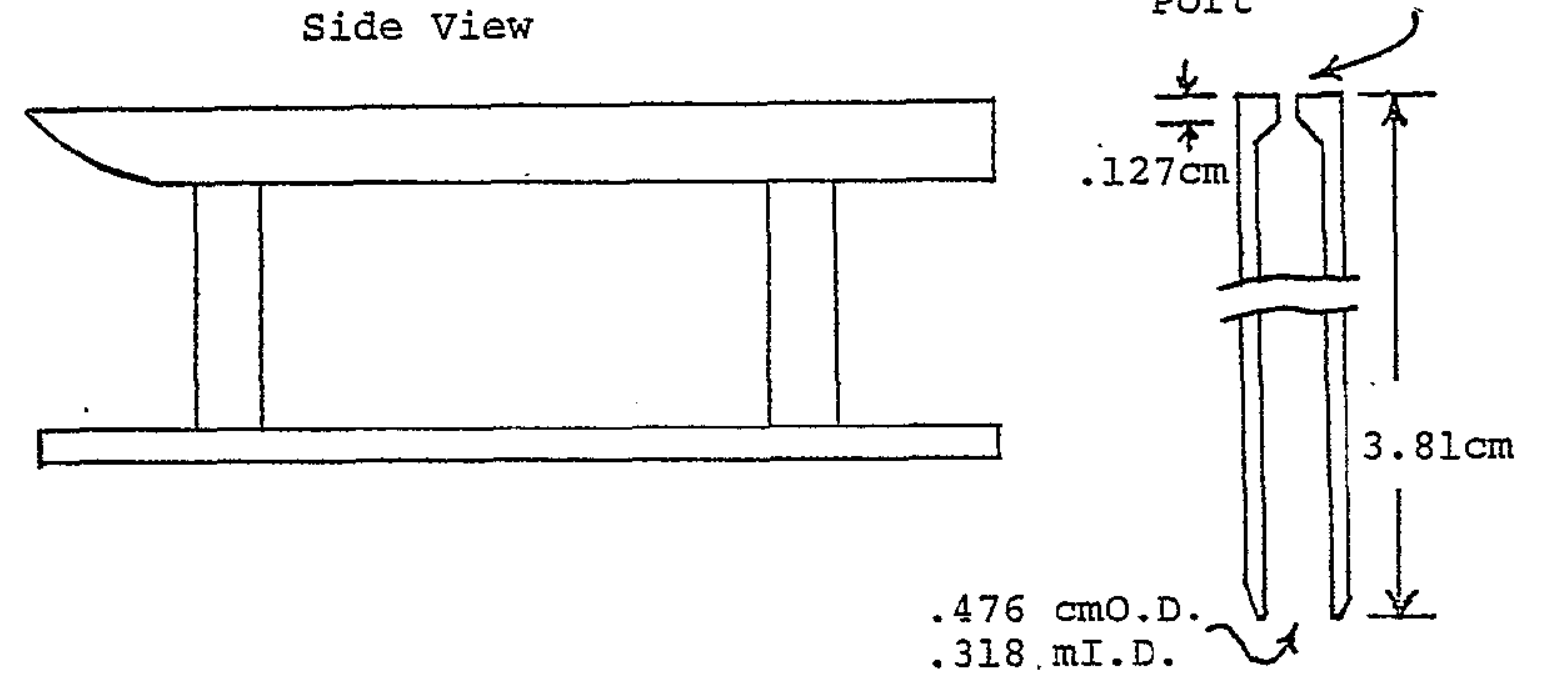

Figure 3 Flat Plate assembiy-Pressure port Locations ( $t$ ) Shown (Half-scale Drawing), and Pressure port Schematic 
The second type of confining surface used is a scaled down version of the wing used at NASA-Iangley in their "Beach" test. The wing or curved wall surface (henceforth denoted "flap") is $15.24 \mathrm{~cm}$ long, $17.78 \mathrm{~cm}$ wide and sweeps out an arc of 70 degrees. The flap is constructed from white pine, with several coats of stain and wax applied to smooth out the surface and make it comparable to the finish of the plate. Figure 4 shows the flap and its mount, which again are placed in the test section of the wind tunnel. Also, the locations of the surface pressure ports are shown. A second point to consider is that the flap does not extend across the entire test section. To determine what effects if any were the result of end effects, several measurements for the flow over the flap were made with extended flanges on both sides of the curved wall structure. With these flanges, the flap extended across the entire test section. No noticeable difference in the measurements was determined.

The inclusion of the plate or flap in the wind tunnel test section necessitates the monitoring of their effects on the outer tunnel flow. A pitot static probe in conjunction with an electronic manometer was used to make mean velocity profiles, traversing from near the top of the test section down to the surface of the flap, then from the underside of the flap to the lower wall of the wind tunnel. The profiles 


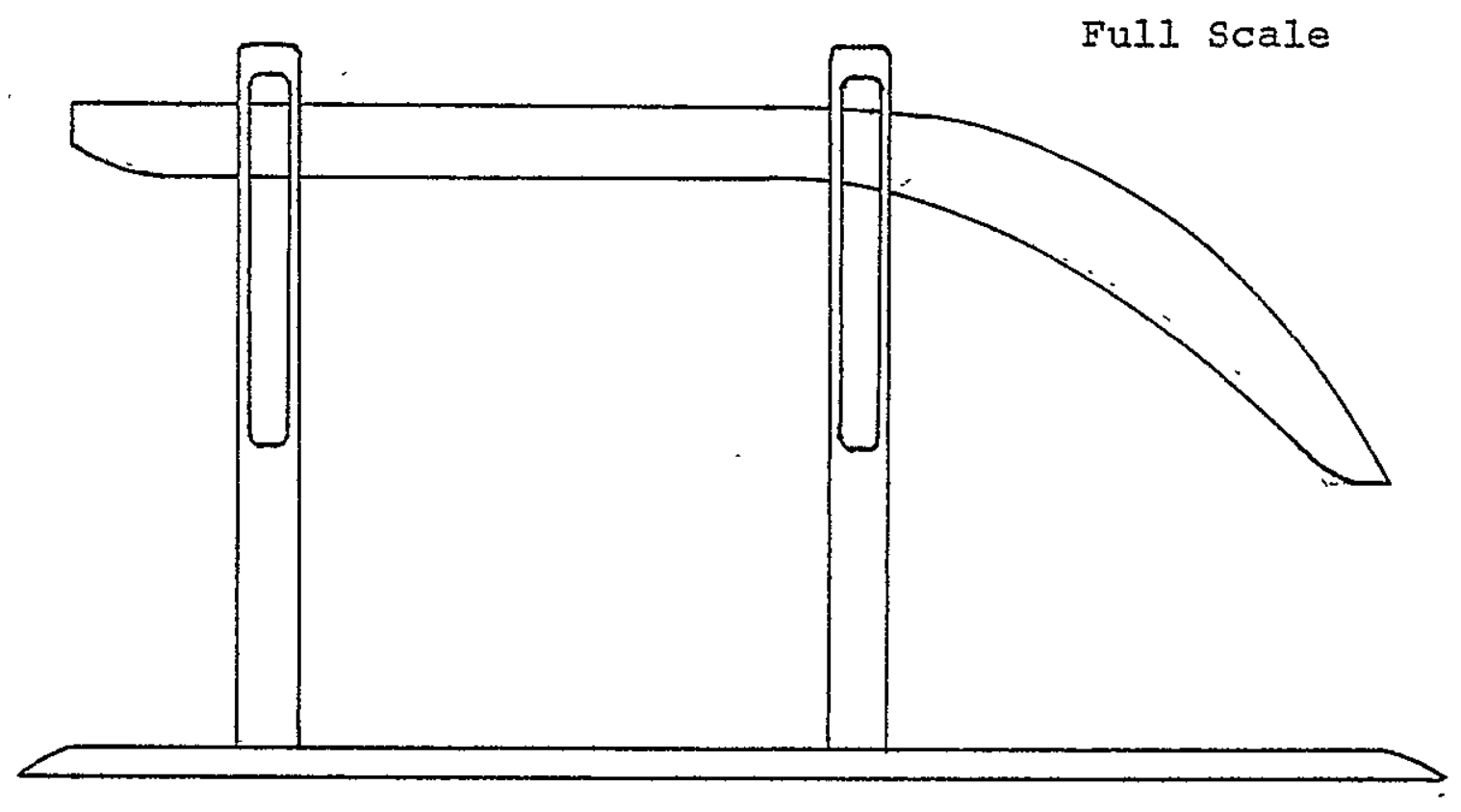

Half Scale

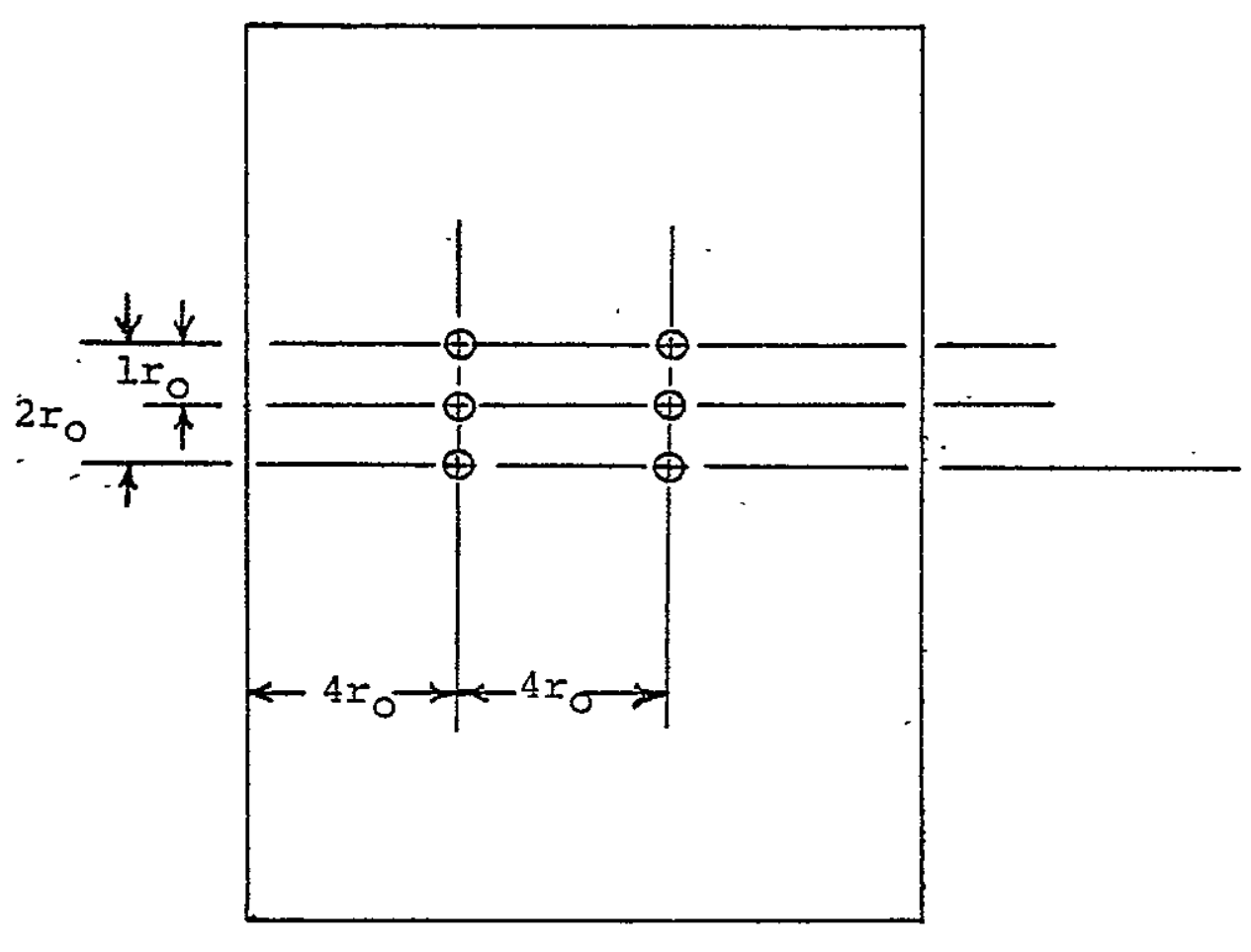

Figure 4 Curved Wall (Flap) Assembly - Pressure Port Locations (+) Shown 
were taken at both values of $\lambda_{j}$, and for both the flow over the plate and the flow over the flap. Examples of the resultant plots are shown in Figure 5. The outer tunnel flow was found to behave as a uniform stream outside the region affected by the jet flow field. Profiles of the outer tunnel flow with the flap in place but the jet off were also determined. Again, the tunnel flow remained uniform outside the nozzle and flap wake region.

The coordinate system used in this investigation is shown in Figure 6(a). In Figure $6(b)$, the jet/flap assembly is shown mounted in the test section of the wind tunnel. The optical components will be discussed at a later time.

Section 3.2 Two Color Iaser Doppler Velocimeter

The light source for both the velocity and intermittency measurements is a Spectra Physics Model 165 Argon Ion Iaser. The diameter at the $1 / e^{2}$ points of the laser beam at its head is $1.5 \mathrm{~mm}$ while in the test section the diameter is approximately $2 \mathrm{~mm}$. The noise level can be reduced to about $0.5 \%$ by tuning. Eight frequencies are present in the alllines-operating mode with the two strongest $(488.0 \mathrm{~nm}$ rated at $700 \mathrm{mw}$ and $514.5 \mathrm{~nm}$ rated at $800 \mathrm{mw}$ ) being selected for use. In order to split the laser light into its different wavelengths, an extra dense flint glass prism is used $(n=1.655)$. 


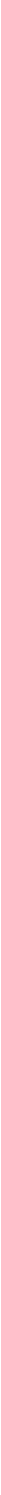

Figure 5 Vertical Plane Pressure Probe Surveys of Jet/Flap

$$
\left(\lambda_{j}=5.1\right)
$$



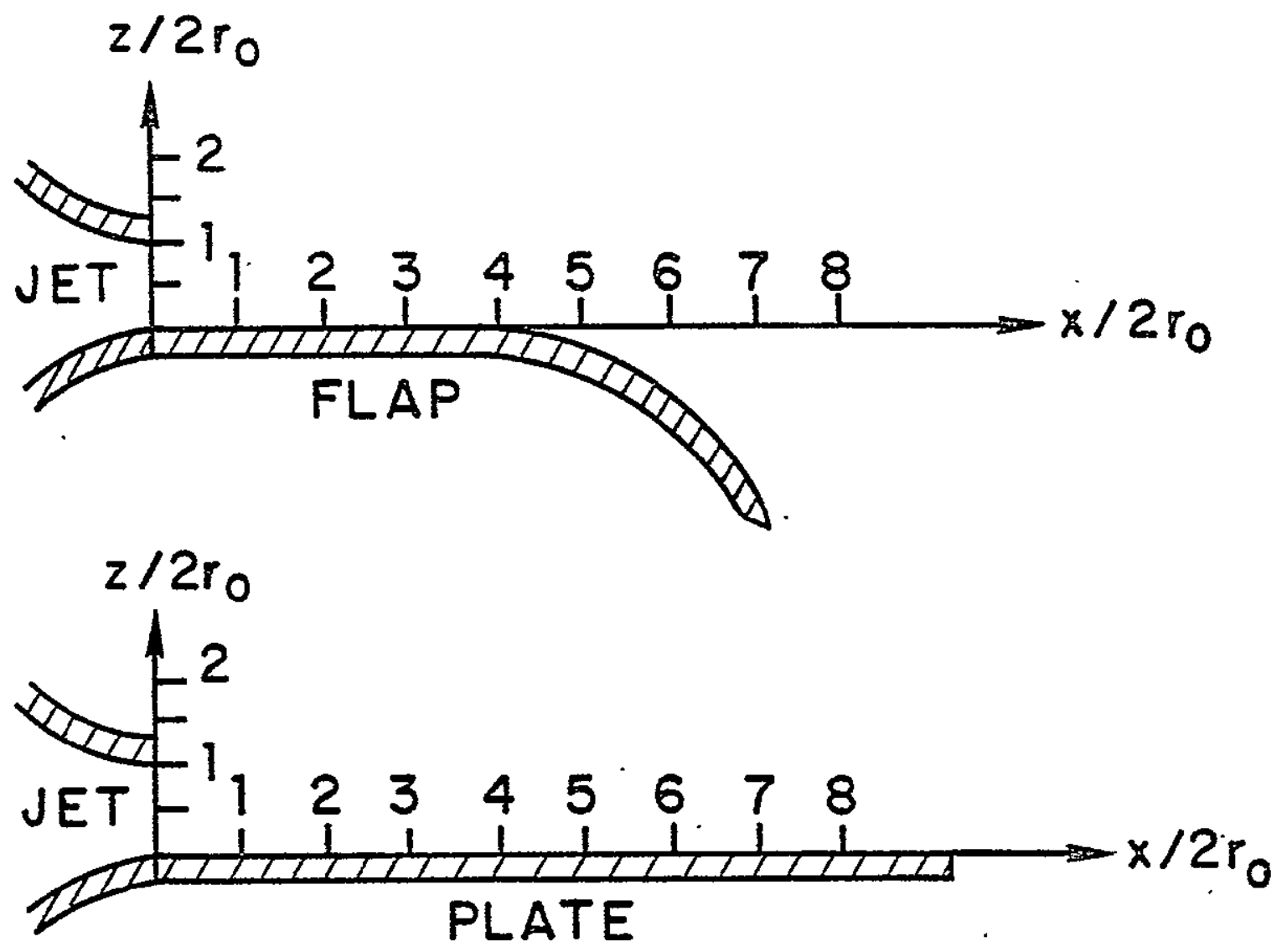

Figure $6(a)$ Jet/Flap and Jet/Plate setup.

Figure $6(b)$ Schematic of Laboratory Facility

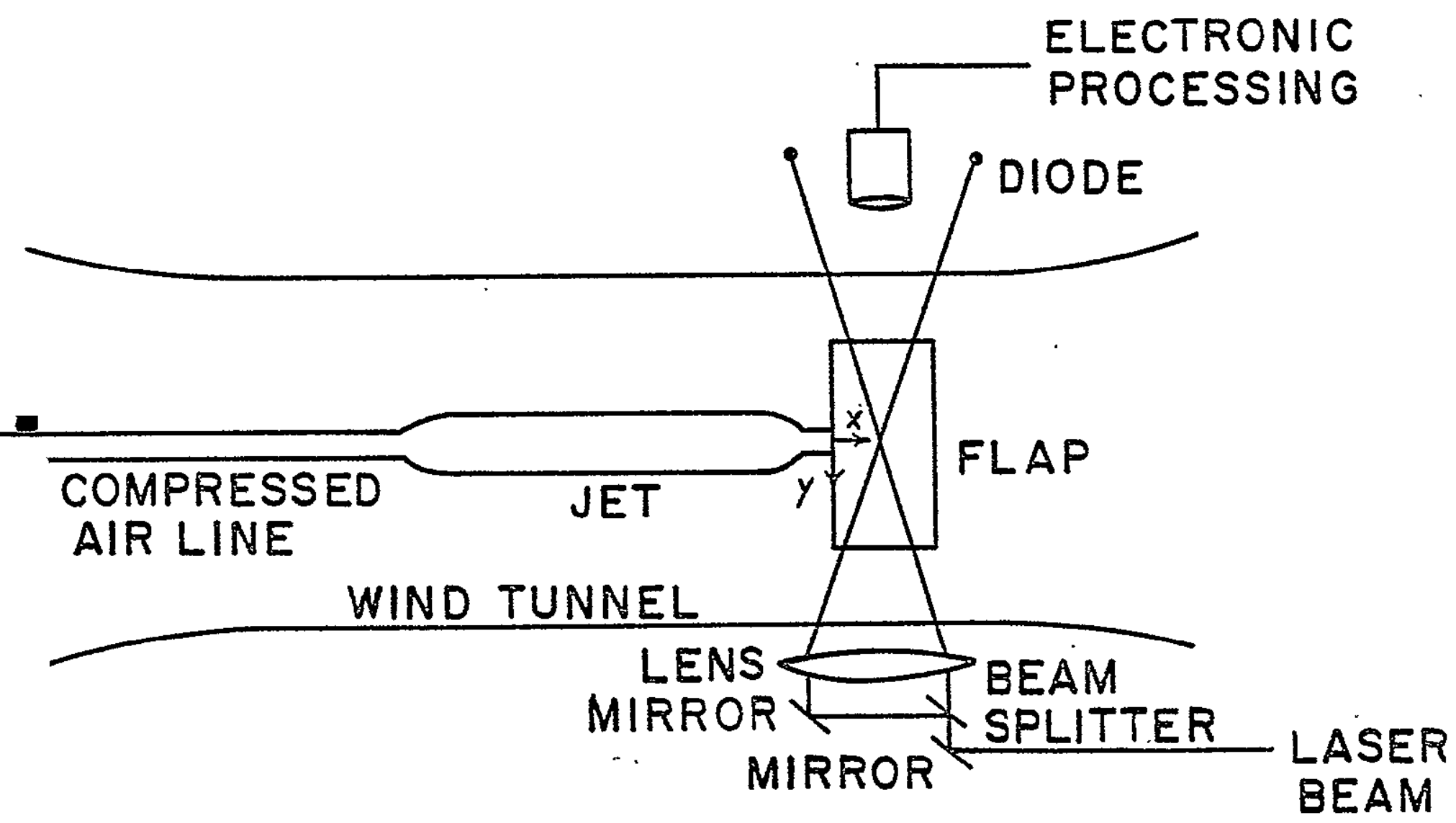


Several views of the different components of the two point measuring assembly are shown in Figures $7(a)-(d)$. In the pictures, the plate is present in the test section. Since the plate is constructed by use of an optical quality. glass flat, a forward scattering (reference beam) mode of light collection can be utilized for the vertical beams. The resultant higher light intensity levels can be detected by using a Bell \& Howell photodiode with an active surface of $1.27 \mathrm{~cm}$. Forward scattering can also be used when the freely expanding coflowing jet is investigated. However, when the model flap is in place, the receiving optics detector assembly must be mounted on the top side of the test section and, hence, a back scattering mode is used.

since the intensity of light collected in the back scatter mode is significantly less than in forward scattering, a photomultiplier tube (EMI-95025) is used due to its greater gain potential. The spatial filter for the photomultiplier tube consisted of a $10 \mathrm{~cm}$ lens mounted immediately above. the upper test section wall and a $400 \mu$ (diameter) pinhole located at the focal plane of the image formed. The choice of the $400 \mu$ pinhole gives the largest signal to noise ratio for the Doppler signal.

For the horizontally directed beams, a forward scattering (dual beam) mode can be employed for all three flow configurations. Once again, a Bell \& Howell diode with an 


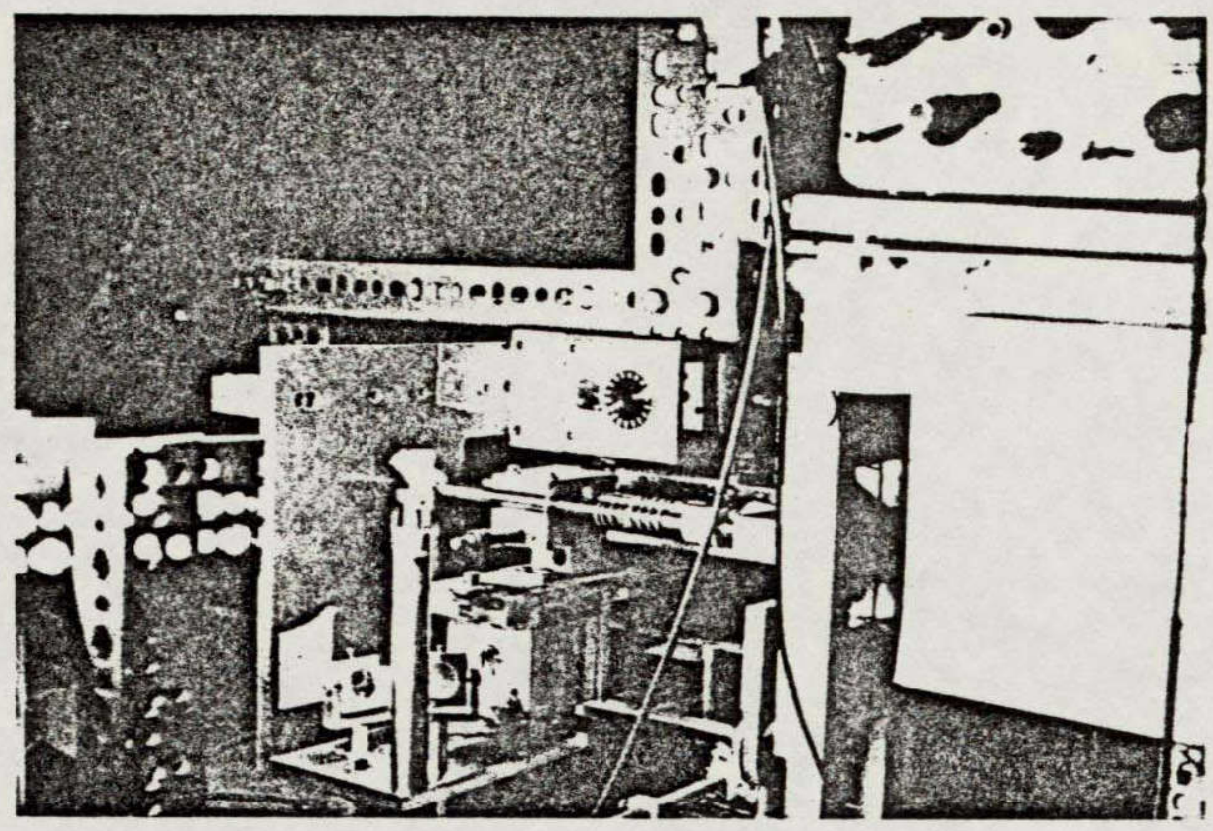

Figure 7 (a) Two-color IDV Setup

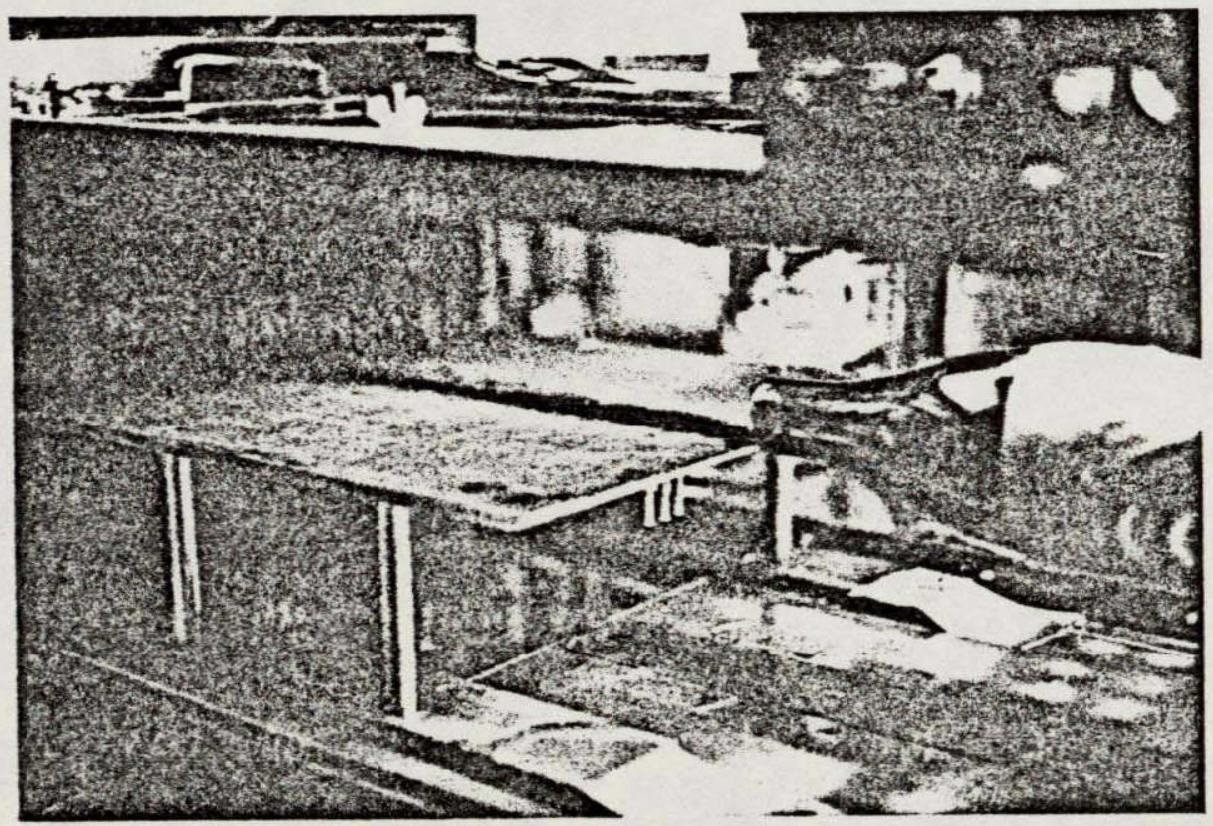

Figure 7 (b) Jet and Plate Installed in Wind Tunnel Test Section (Note outline of jet on surface of the plate) 


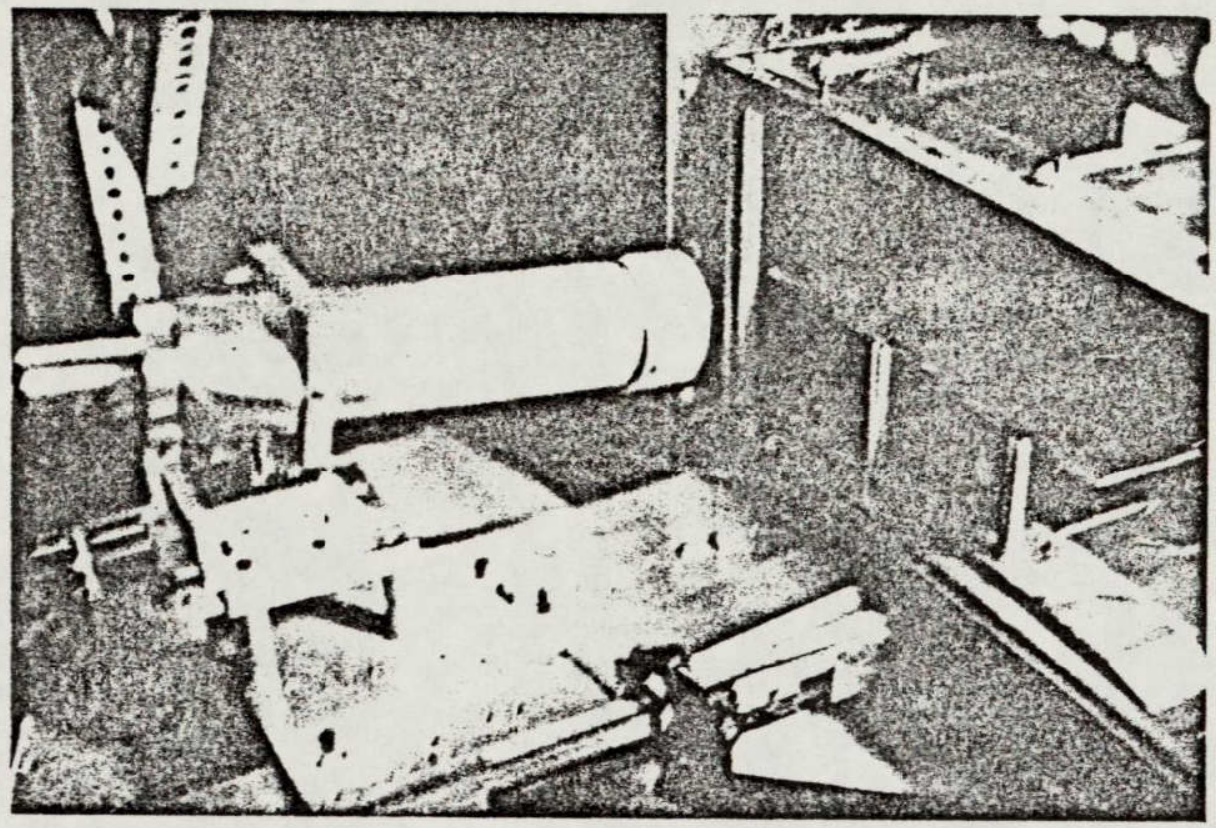

Figure 7 (c) Dual Beam Receiver/Detector (Diode)

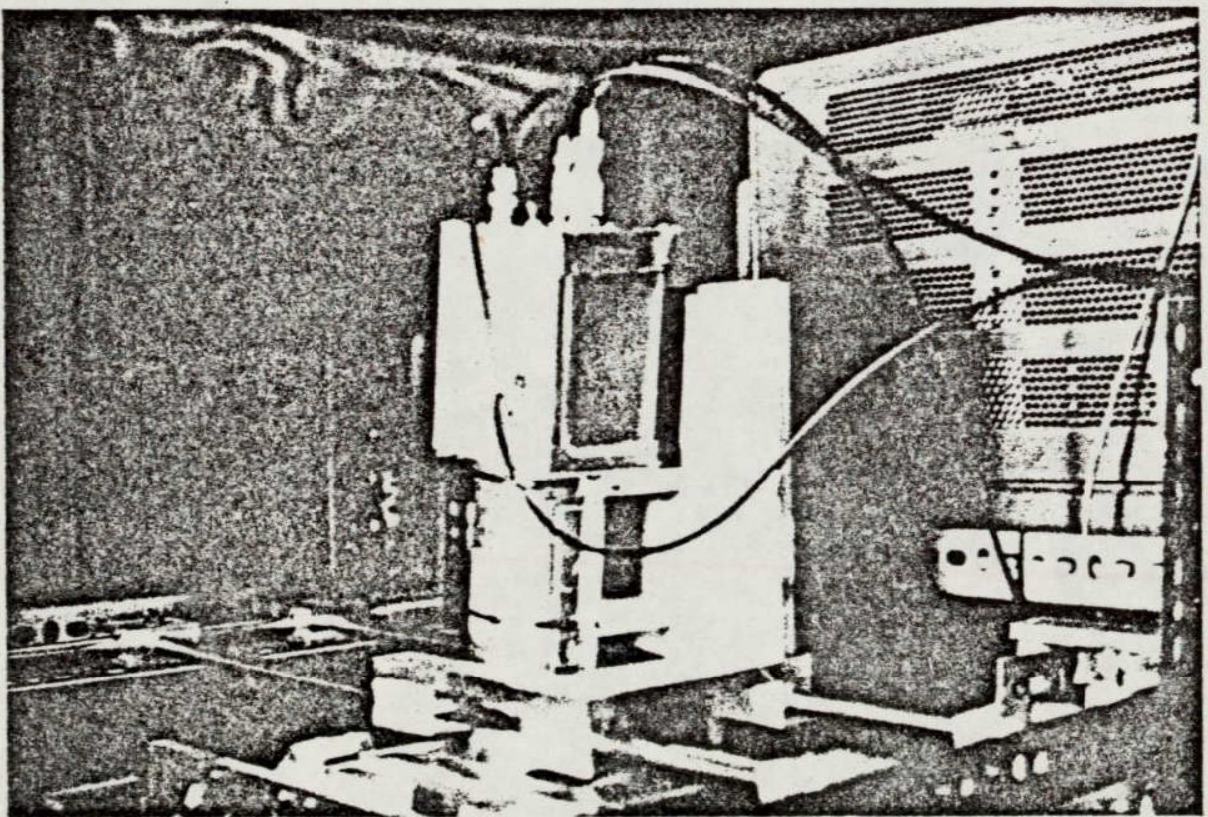

Figure 7 (d) Back Scattering Receiver/Detector (Photomultiplier tube) 
active surface diameter of $1.27 \mathrm{~cm}$ is used. To increase the signal to noise ratio of the Doppler signal, a spatial filter consisting of a lens with a focal length of $10 \mathrm{~cm}$ and an aperture with a $500 \mu$ diameter located at the focal plane of the image formed is utilized.

Both optical units or "boxes" are identical in construction and components. The optical components consist of a rhombiod shaped prism, a beam splitter, a mirror and a lens. The rhomboid prism allows the rotation of the entire unit about the optical center. With this capability, the plane of the two intersecting beams can be adjusted so as to give transverse as well as longitudinal velocity information. The beamsplitters are rated at 70-30 (i.e. $70 \%$ of laser beam passes through while $30 \%$ is reflected). The focal length of the lens is $30 \mathrm{~cm}$ which produces a control volume size of approximately .05 mm (diameter) $\times 1$ mn (length). (55) For the reference beam mode of operation, a \#2 neutral density filter is used to reduce the intensity of the beam which hits the photodiode surface.

The entire optical assembly is mounted on an alumimum slide which provided movement of both laser "probes" in a horizontal direction perpendicular to the jet and tunnel flow axes. Additionally, the horizontal beam unit can be moved independently to allow a displacement of the control volume in all three directions. 
The two color IDV arrangement proved to be extremely effective in determining the one and two point statistical properties of the flow. For one point properties, flexibility is inherent in the system because when an opaque surface is encountered with one "probe", the use of the other laser measuring device allows the experimentalist to always use a forward scattering technique. The data obtained from the different "probes" at the same location in the flow field were remarkably similar except in the cases when sharp gradients in the flow field properties occurred (Appendix A) . Two point velocity measurements require a very precise determination of the separation between the sensitive control volumes. Critical to this determination is knowing when the "probes" are measuring at the same point in the flow field. To accomplish this alignment, use is made of the Federal Scientific Ubiquitous Correlator.

The fluctuating voltage (velocity) signals from both of the "probes" are correlated. A relative downstream displacerient between the control volumes would manifest itself as a shift in.the maximum value of the cross-correlation from a zero delay time. Any lateral displacement would cause a skewness in the cross-correlation curve and/or a decrease in magnitude of the value of the covariance at zero delay time. This trial and error procedure, though somewhat tedious, allows very precise positioning of the laser control volumes. 
Once the control volumes are precisely located, and the scattered light is collected by the photodiodes and/or photomultiplier tube, the resultant information is then analyzed by the phase locked-10op processor. (PLI) The PLI is discussed in detail in Appendix C. The two PLI's used in this investigation are constructed of identical components and their respective output signals are in phase with each other.

A schematic of the complete electronic equipment set-up is shown in Figure 8 .

With the PLI properly set so that it is centered at the mean frequency of the flow, the signal from the detector is disconnected. A sine wave at the same frequency from a Tektronix Type 190 B Signal Generator is then connected to the PLI, and the mean frequency is read using a Hewlett Packard Type 5216A Electronic Counter. This procedure was adopted since it allowed the mean frequency to be determined much more accurately than was possible by other methos. Once the detector signal is reconnected, the voltage error signal from the PLI is utilized. Root mean square voltages are measured with a Disa Type 55D35 Rms Voltmeter. The voltmeter has a frequency range of $1 \mathrm{~Hz}$ to $100 \mathrm{KHz}$, and signals with crest factors as high as $15 \mathrm{can}$ be measured. In this investigation, the compressed air jet is marked with DOP while the secondary tunnel flow is kept clean. The phase locked loop is triggered only if marker particles are present in 


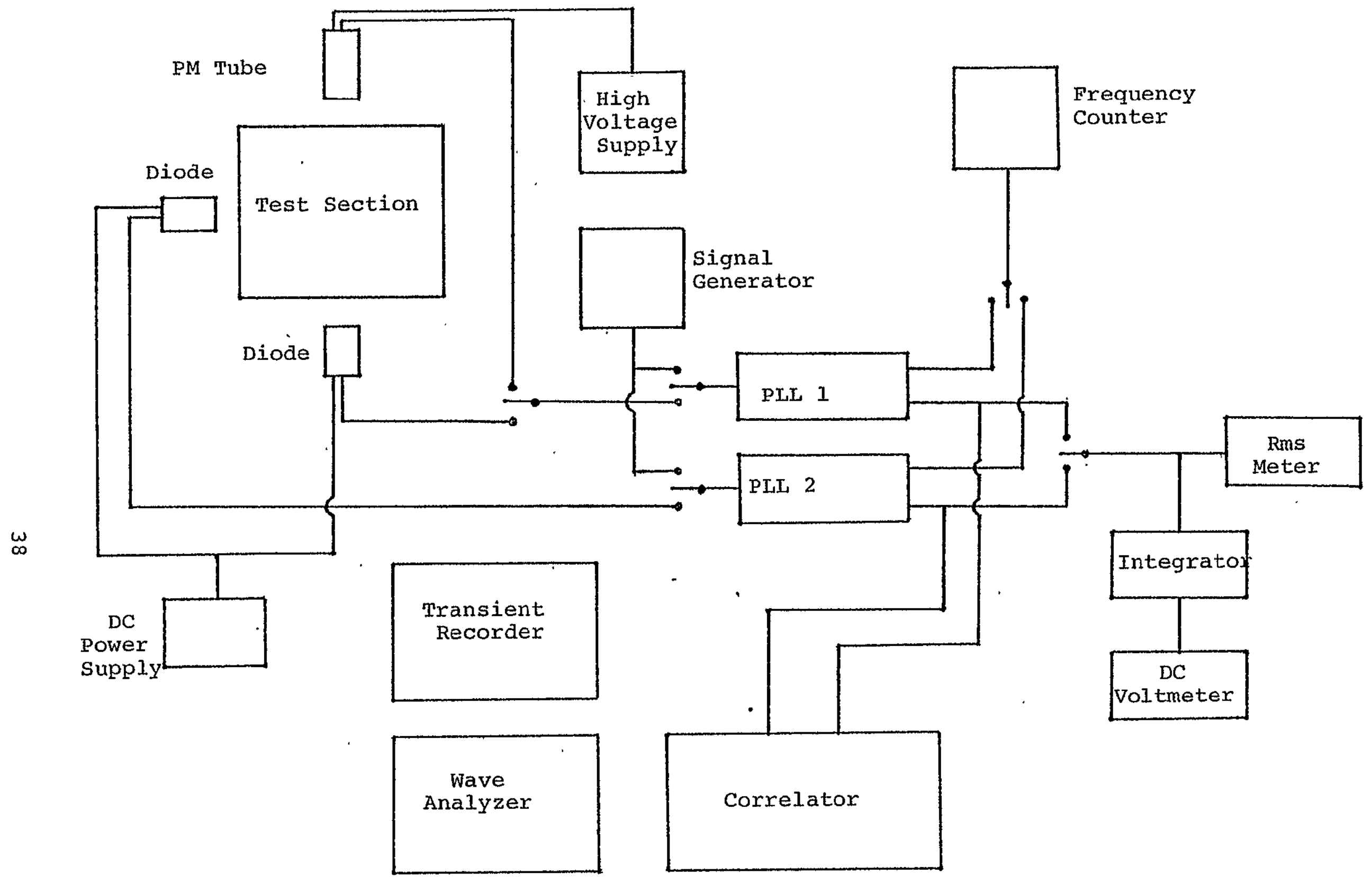

Figure. 8 Schematic of Electronics Setup 
the control volume. Thus, due to the high schmidt number $\left(4 \times 10^{4}\right)$ of the flow, the phase locked loop determines the velocity field of the jet.only." Measurements.taken in this manner are considered "conditionally sampled".

To obtain autocorrelation and cross correlation curves and coefficients, the voltage error signal(s) are analyzed by use of Federal Scientific Model UC-202B Ubiguitous Correlator. From the autocorrelation curves, Taylor microscales and integral scales can be determined. The microscales are evaluated by numerically fitting a parabola at the origin of the curve, while the integral scales are determined by a numerical integration of the area under the autocorrelation plot. A computer program was developed which enables the power spectral density function to be obtained from the autocorrelation curves by using a Fourier Transform technique.

The Biomation Model 8100 Transient Recorder and the Nelson Ross Electronics Spectrum Analyzer were quite useful in determining the quality of the Doppler signal. The transient recorder, which stores the digital equivalent of electrical waveforms in a memory, was used to make histograns, to take pictures and to make plots of the individual Doppler bursts.

For the intermittency measurements, the cumulative probability density mode of the correlator is used. The cumulative probability density (CPD) is defined as the 
fraction of time the signal is less than or equal to a certain voltage. If the CPD of the concentration signal was measured at zero voltage, one minus this fraction would be the intermittency. Actually, the voltage for which the CPD is $99 \%$ is measured in order that only the percentage of time the concentration signal exceeds the noise is determined.

Section 3.3 Pressure Measuring System and pu Correlations

To determine the static pressure and the wall sufface pressure, the system developed by schroeder ${ }^{(57)}$ and Herling ${ }^{(58)}$ was used. The essential items included a 1/2 inch condenser type microphone and a Nagra IV-SJ tape recorder. When the cross correlations were made between the pressure and velocity fluctuating fields, both signals were filtered ( $10 \mathrm{~Hz}$ - $1000 \mathrm{KHz}$ ) before being processed in order to achieve a good signal to noise ratio. The filters used were DISA 55D25 Auxilary units.

A computer program was used that enabled the spectra of both the pressure and velocity to be obtained as well as the coherence between the two signals. Coherence is essentially the value of the cross-correlation coefficient as a function of frequency. If $G_{i 1}$ and $G_{12}$ denote the Fourier Transform of the autocorrelation function of Input 1 and Input 2 (in this investigation, pressure and 
velocity); then the coherence, $\delta_{12}^{2}$, is defined as follows:

$$
\delta_{12}^{2}=\frac{\left|G_{i 2}\right|^{2}}{G_{11} G_{22}}
$$

where $G_{12}$ represents the-Fourier Transform of the crosscorrelation function of Input 1 and Input 2 .

\section{Section 3.4 Intermittency Measuring System}

Intermittency is customarily defined as the percent time the flow is turbulent at a given location in the field. In this investigation, the compressed air jet flow was marked with dioctyl phthalate (DOP) while the tunnel flow was kept clean.' Since the schmidt number, which is defined as the ratio of the kinematic viscosity of the flow to the coefficient of the diffusivity of the DOP smoke particles, is very high $\left(10^{4}\right)$ the smoke remained confined to the jet. The only way that smoke particles would be at a location in the flow is if the turbulent jet is also present.

The only way clean air could be marked by the DOP was by being entrained into the jet flow. Thus, outside the potential core region, the presence of smoke indicated the presence of turbulence.

To determine the intermittency profiles, the system developed by Shaughnessy is utilized. (59) The design includes a lens-mirror assembly which permits the selection of virtually any size control volume from . I mm by $.1 \mathrm{~mm}$ by $: 1 \mathrm{~mm}$ to $1.5 \mathrm{~mm}$ by $1.5 \mathrm{~mm}$ by $1.5 \mathrm{~mm}$. The lens has a 
focal length of $20 \mathrm{~cm}$. The detection system uses a PIN silicon photodiode with an active surface diameter of .5 mm mounted behind a spatial filter. The spatial filter assembly is composed of a simple lens and an aperture located in the focal plane of the image found. The lens of this spatial filter assembly is positioned for unit magnification.

Section 3.5 Concentration Measuring System

To make the concentration fluctuation measurements, a small modification is made to the two point IDV setup. The back scattering collector/receiver is kept the same but the current caused by the scattering of laser light by the particles is monitored. In the dual beam, forward scattering mode, the spatial filter is similar except for the addition of a $400 \mu$ aperture. Also, the Bell and Howell photodiode is replaced by a PIN silicon photodiode with an active surface of $.51 \mathrm{~mm}$. It should be pointed out that both receiver/detectors are positioned so that they only monitor the light being scattered by the DOP particles as the smoke passes through the control volume. Auto and cross correlation curves are obtained by use of the Federal scientific correlator. The power spectra are obtained by numerically Fourier Transforming the autocorrelations. 


\section{Chapter 4}

\section{EXPERIMENTAI RESUITS AND. DISCUSSION}

One and two point statistical properties are measured in the flow fields of a coflowing turbulent jet, a coflowing turbulent jet and plate, and a coflowing turbulent jet and flap. The one point statistical properties include mean velocities and turbulent intensities in all three directions, autocorrelations and power spectra of the fluctuating velocity field and the concentration field, and intermittency profiles of the passive admixtive flow field. From the autocorrelation curves, the Taylor microscale and the integral length scale are calculated using Taylor's hypotheses ${ }^{(1)}$. The mean velocity profiles for the flows over the flap and plate are compared to existing theories when possible. The effects of varying the ratio of the inner jet velocity to the outer tunnel speed are also noted.

The two point statistical properties measured include space-time autocorrelations of the three different velocity fields, space time autocorrelations of the concentration field and pressure-velocity correlations between various points in the turbulent velocity flows and pressures measured in the flow fields at the upper surfaces of both the plate and the flap and at a sting mounted 
static probe. The space-time autocorrelation coefficients are used to construct iso-correlation contours. These isocorrelation contours yield a considerable amount of information concerning the effects of a confining surface on the structure in the near field of a turbulent jet.

All velocity measurements are made with a laser Doppler velocimeter, the intermittency data is obtained by use of a laser light scattering technique, and the pressures are monitored with a $1 / 2$ inch condensor type microphone.

Section 4.0 Mean Velocities

In Figure 9, mean velocity profiles for the longitudinal component are presented for two downstream locations $\left(x / 2 r_{0}=2,4\right)$, and at one vertical position $\left(z / 2 x_{0}=0.5\right.$, the coflowing jet centerline $)$ for all three flow fields with $\lambda_{j}=5.1$. The ratio of the local excess velocity, U - U US' to the excess core velocity, $U_{0}-U_{F S}$, is plotted versus lateral displacement (non-dimensionalized by twice the radius of the jet) from the centerline of the jet nozzle.

Initially, consider the comparison of the width of the three flow configurations. The freely expanding jet and the flow over the plate are quite comparable in width both at two and four diameters downstream. However, the 


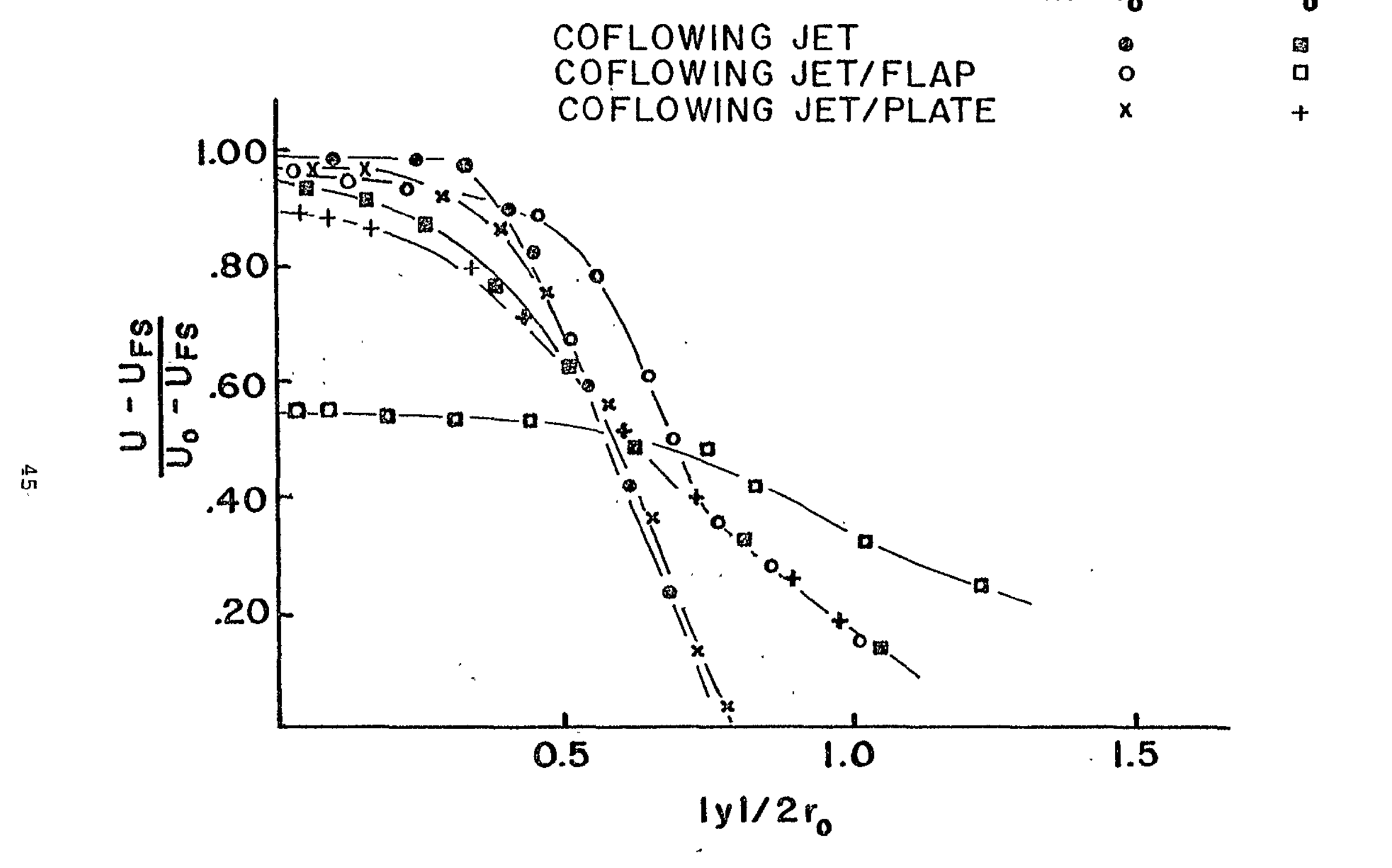

$x / 2 r_{0}=2 x / 2 r_{0}=4$

COFLOWING JET

COFLOWING JET/FLAP

政

COFLOWING JET/PLATE

$x$

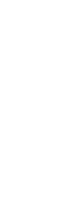


flow over the flap has a significant increase in width compared to the previous configurations at both downstream locations. Secondly, notice the effect of the confining walls on the decay of the core velocity. The presence of either the flap or plate serves to break up the mean velocity field in the potential core region more rapidIy. The flap is, however, much more effective. Thus, as the turbulent jet flows over the flap, the velocity field is widened and decelerates much more rapidly. The observation concerning the effects of the two surfaces on the width of the jet is quantified in Figures $10(a)$ and $(b)$. Here $y_{m} /$ ro is plotted versus $x / 2 r_{0}$ for $z / 2 x_{0}=0.5$. The quantity, $Y_{m^{\prime}}$ is defined as the lateral distance from the centerline to the location where the mean velocity is the arithmetic average of its value on axis and in the secondary stream. Data for both ratios of inner jet velocity to tunnel speed (i.e. $\lambda_{j}=5.1$ and $\lambda_{j}=10.88$ ) are presented, and the results compared to a theoretical curve developed by squire and Trouncer ${ }^{(60)}$ for the expansion of a coflowing jet $\left(\lambda_{j}=5\right)$. First, consider the findings for $\lambda_{j}=5.1$. While the agreement between the theoretical curve and experimental results for the coflowing jet is quite good, one effect of the confining surfaces can be seen. For the flow over the flap, the value $\mathrm{y}_{\mathrm{m}}$ increases much more 
$\mathrm{ym} / \mathrm{ro}$

$\mathrm{ym} / \mathrm{ro}$ (a) $\lambda_{j}=5.1$

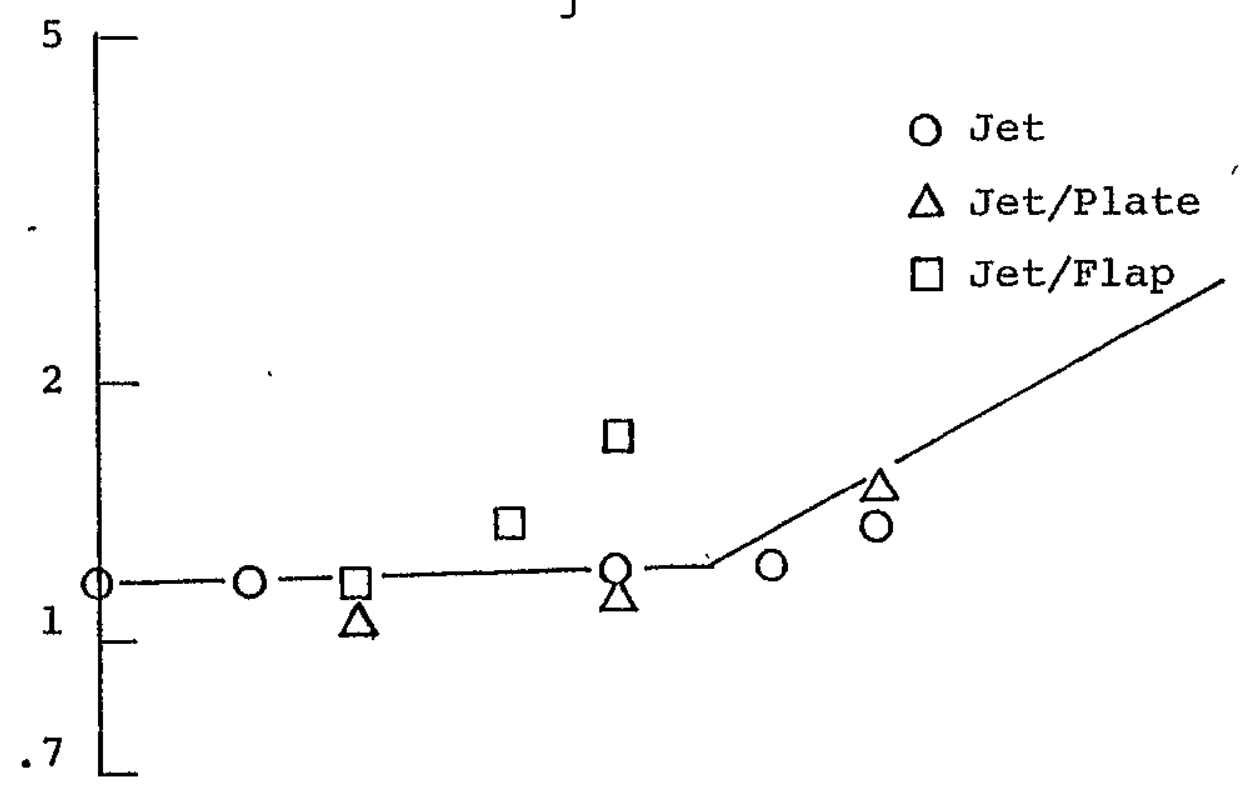

$\stackrel{\leftrightarrow}{4}$

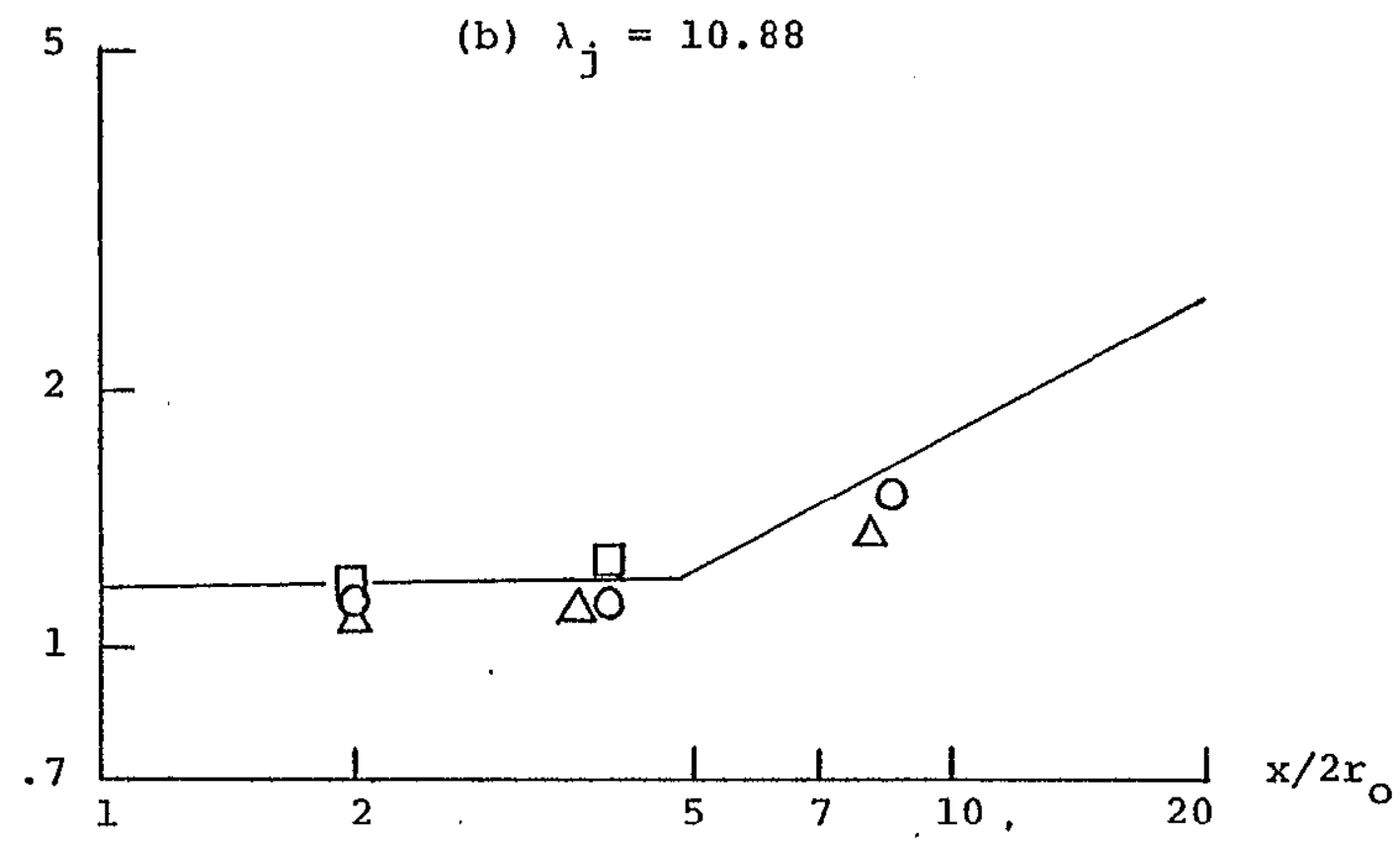

Figure 10 Growth of Mixing Width (Squire and Trouncer: -) 
rapidly than is, the case for the other two configurations. With the plate in place, however, the value of $\mathrm{ym}$ is somewhat, smaller than that for the coflowing jet for the first several diameters downstream but eventually is quite comparable in magnitude. For $\lambda_{j}=10.88$, the results are somewhat less clear. Though the value of $y_{m}$ for the flow over the flap still seems to increases the most rapidiy, the rate of increase is considerably less than is the case for $\lambda_{j}=5.1$. It is difficult to draw any stron conclusions, however, since the data is somewhat sketchy. Here, also the mixing widths of the unconfined jet and flow over the plate are quite comparable in magnitude.

In Figures $I I(a)$ and (b), the decay of the centerline mean velocity at $z / 2 r_{0}=0.5$ for varying downstream locations is presented. The meaning of the data for the two jet/confining surface configurations should be discussed. As the flow exits the circular nozzle, the plate and the flap serve to transform the "top hat" mean velocity profile, characteristic of an ideal jet, into profiles more closely resembling wall jets. Thus, the velocity at the nozzle centerline would be expected to decrease at a different rate for the jet/plate and jet/flap cases as compared to the unconfined coflowing jet case. Secondly, considering the curvature of the flap, if this curvature were to cause a change in the direction of the flow, a decrease in the mean velocity 


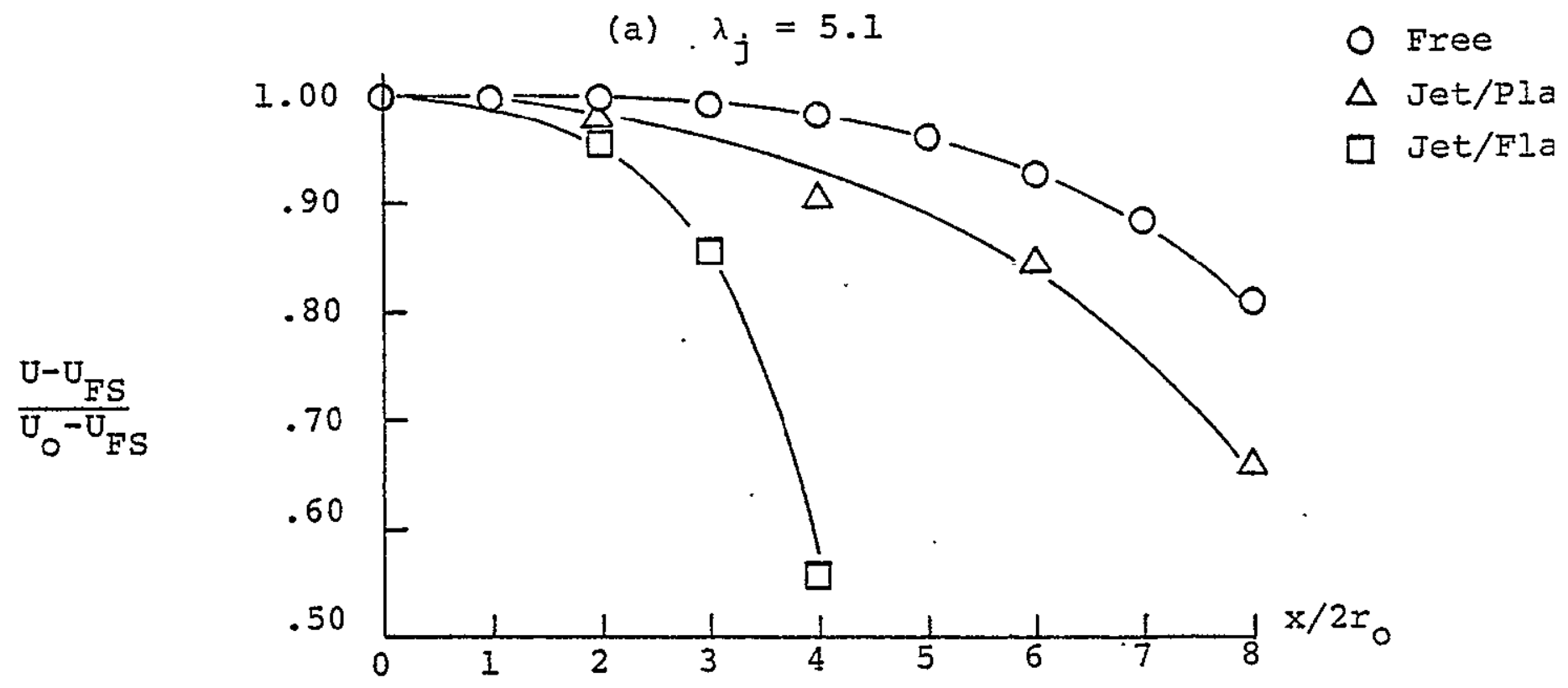

(b) $\lambda_{j}=10.88$

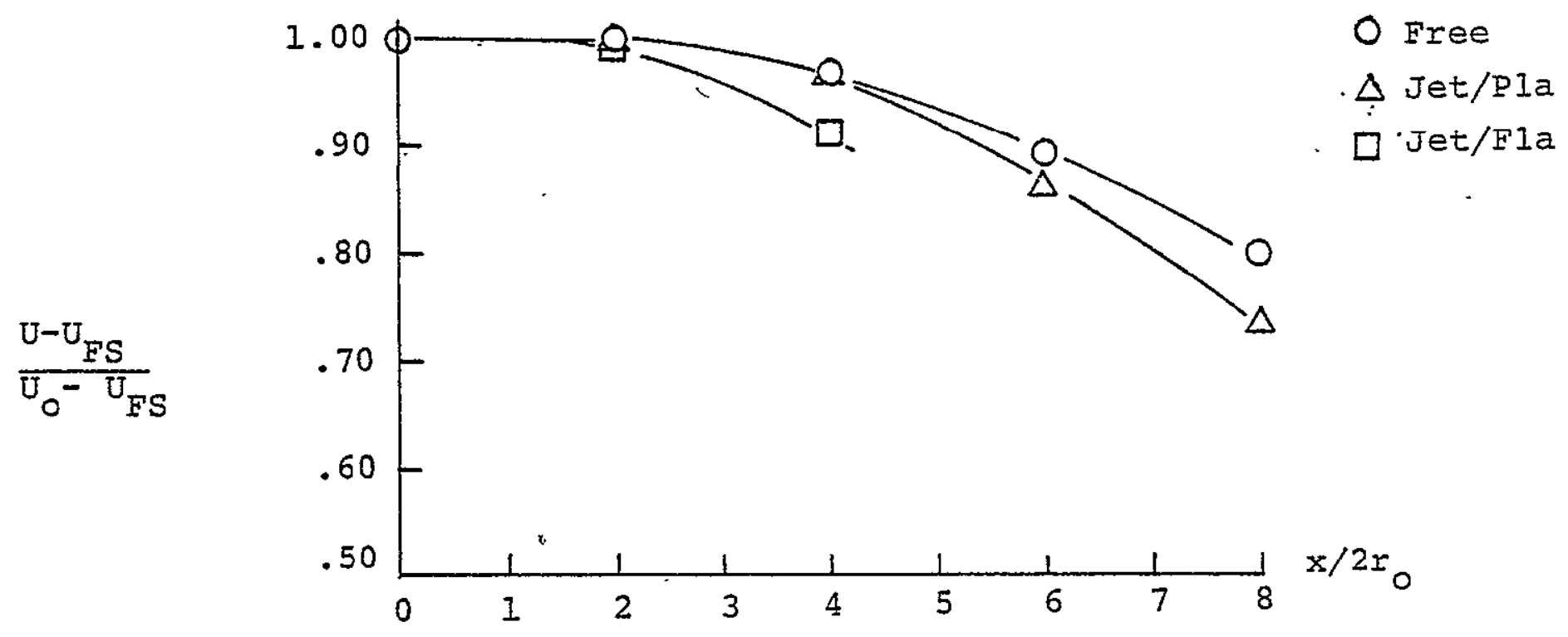

Figure 11 Decay of Centerline Mean Velocity,
$z / 2 r_{0}=0.5$ 
in the $x$ direction would be the result. For the downstream locations shown, however, the mean velocity has not changed its direction by any large amount from the $x$ direction. For example, at $x / 2 r_{0}=4$ with $\lambda_{j}=5.1$, total mean velocity vector acts at an angle of $-7.1^{\circ}$ with the longitudinal direction.

For $\lambda_{j}=5.1$, the effectiveness of the flap in decelerating the flow in the x-direction is apparent. The more rapid decay of the flow over the plate as compared to the unconfined jet is also noted. When $\lambda_{j}=10.88$, the decay of the unconfined jet behaves quite similarly. to the case when $\lambda_{j}$ has the smaller value. The plate is slightly less effective in decelerating the flow while the effectiveness of the flap is reduced significantly.

The two observations that have been made concerning the relative rates of decay of the centerline mean velocity and the increase in the mixing widths for the flows over the flap with $\lambda_{j}=5.1$ and $\lambda_{j}=10.88$ are consistent and merit some discussion. The indication seems to be that, as far as the mean velocity field is concerned, as the value of $\lambda_{j}$ gets larger and approaches the value corresponding to a free jet $\left(\lambda_{j}=\infty\right)$, the flap becomes less effective in both widening and decelerating the flow. 
It should be mentioned that the location $x / 2 r_{0}=4$, corresponds to the point where the flap starts to curve. Since the flow field, with $\lambda_{j}=5.1$, increases in width much more rapidly, the energy required for the acceleration of the slower moving fluid particles would necessarily cause a reduction in the momentum of the flow somewhere else. Hence, to some extent a widening of the flow field would be responsible for the more rapid deceleration which has been observed for the case when $\lambda_{j}=5.1$.

To explore the effects of the curvature of the flap for the flow with $\lambda_{j}=5.1$, mean velocity components in all three directions are measured. These measurements are obtained for locations over both the flat and the curved segments of the flap. Mean velocity parallel to the surface of the flap, $U^{*}$, versus perpendicular height above the surface of the flap, $n$, is presented in Figure 12. An interesting observation that can be made concerning this series of profiles is that $\mathrm{U}^{*} / \mathrm{U}$. has approxiately the same maximum value at $x / 2 r_{0}=.4$ as at $x / 2 r_{0}=2$. This seems to indicate a net transfer of momentum from. the core of the jet down towards the surface of the flap. Also, for the first four diameters downstream, the flow seems to be tending toward the classical wall jet mean velocity profile. This will be discussed in more detail 


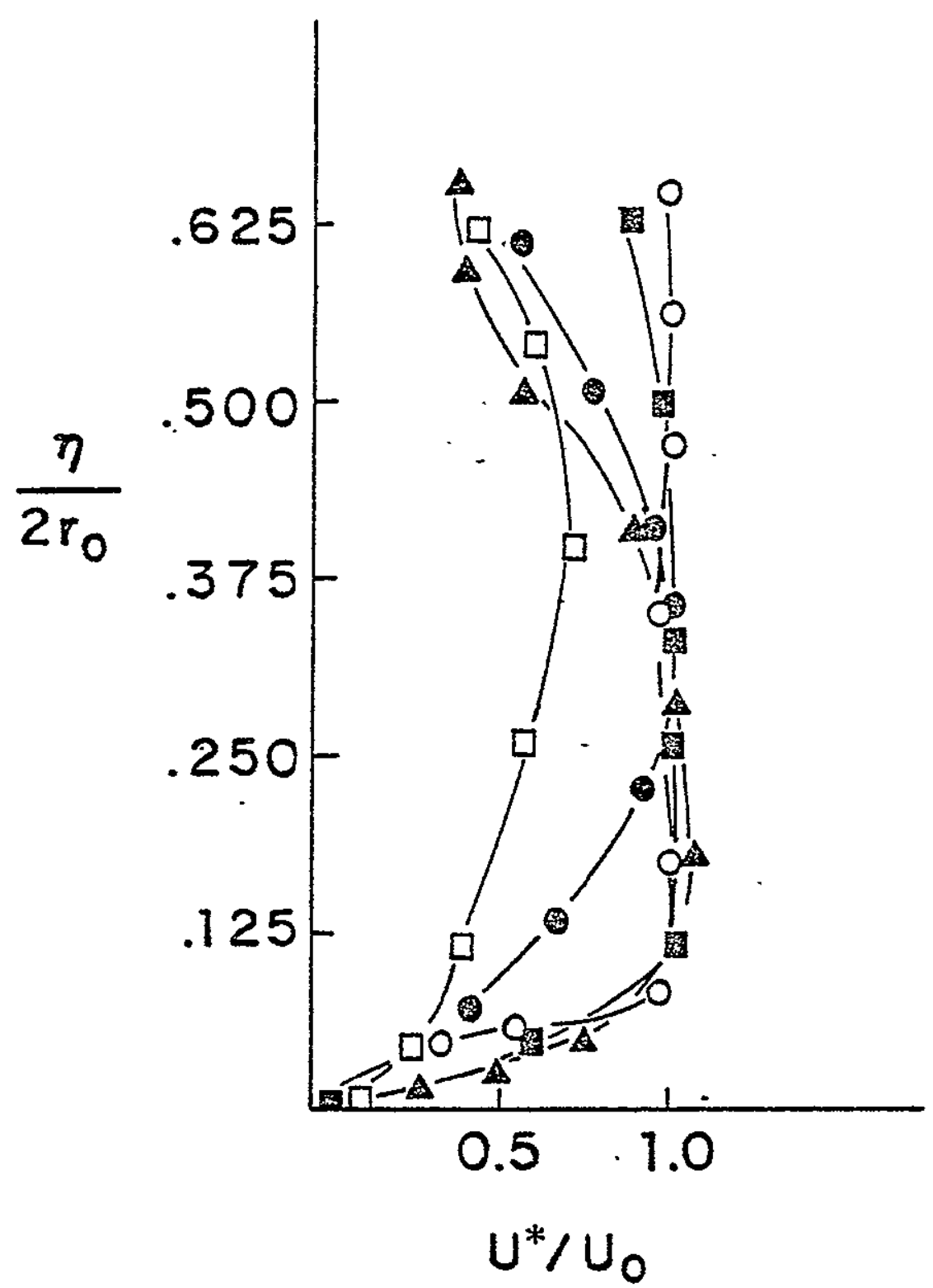

$$
\begin{aligned}
& \text { COFLOWING JET FLAP } \\
& 0 x / 2 r_{0}=2 \\
& \text { 面 } x / 2 r_{0}=3 \\
& \Delta x / 2 r_{0}=4 \\
& \text { - } x / 2 r_{0}=5 \\
& \square x / 2 r_{0}=5.5
\end{aligned}
$$

Figure 12 Development of Mean Velocity Profile in Vertical Plane, $y / 2 \ddot{r}_{0}=0$

$$
\left(\lambda_{j}=5.1\right)
$$


in a later section of the report. However, after $x / 2 r_{0}=4$, any similarity between the measured velocity profile and. the theoretically predicted self-preserving shape of the classical wall jet is no longer present.

Mean velocity profiles in the vertical, $W$, and in the longitudinal $U$, directions for various downstream locations $\left(x / 2 r_{0}=5.5,6.0\right.$, and 6.6$)$ and at the vertical position, $z / 2 I_{0}=-0.57$ are presented in Figure 13 . The effectiveness of the flap in turning the flow is indicated by these profiles in both a qualitative and quantitative manner. At the centerline of the profiles, for example, the ratio of $U$ to $W$ varies from 1.45 to 1.17 to 1.8 as the flow progresses downstream. These values of $\mathrm{U} / \mathrm{W}$ would correspond to angles of $34.6^{\circ}, 40.52^{\circ}$, and $29.0^{\circ}$ with respect to the $\mathrm{x}$ direction.

Figure 14 presents a vectorial diagram of the resultant mean velocities at the centerline $\left(|y| / 2 x_{0}=0\right)$ as the flow proceeds downstream. The angles noted are with reference to the $\mathrm{x}$ direction, and correspond to the direction of the mean velocity vector at each downstream Iocation.

Continuing with the discussion of the flow over the flap, a profile of the mean velocity, $\dddot{w}$, in the vertical direction, $z$, for the downstream location $x / 2 x_{0}=4$ and $z / 2 x_{0}=1.85$ is presented in Figure 15. Comparing this 


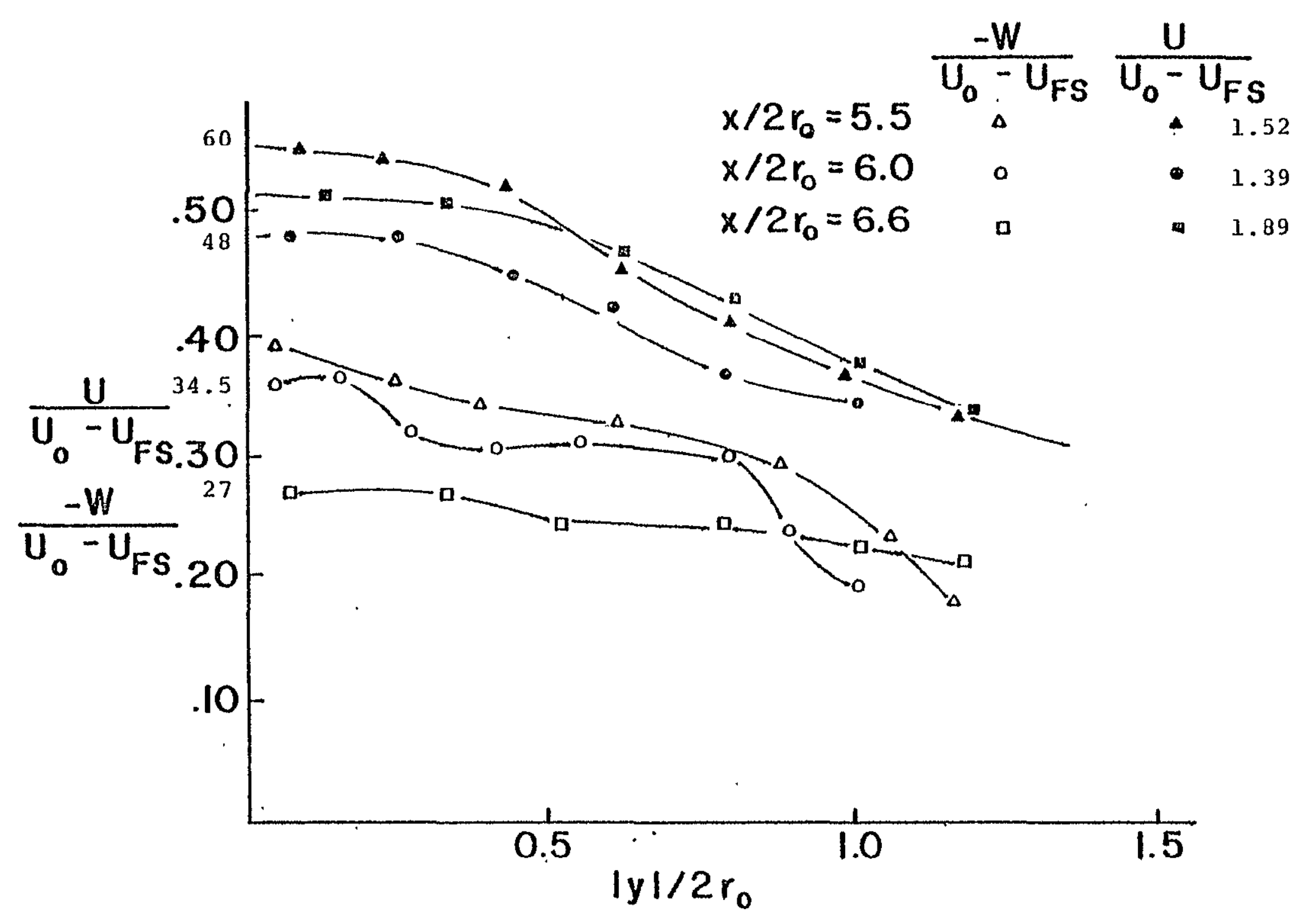

Figure 13 Longitudinal and Vertical Mean Velocity Profiles for Flap, $z / 2 r_{0}=0.57 \quad\left(\lambda_{j}=5.1\right)$ 


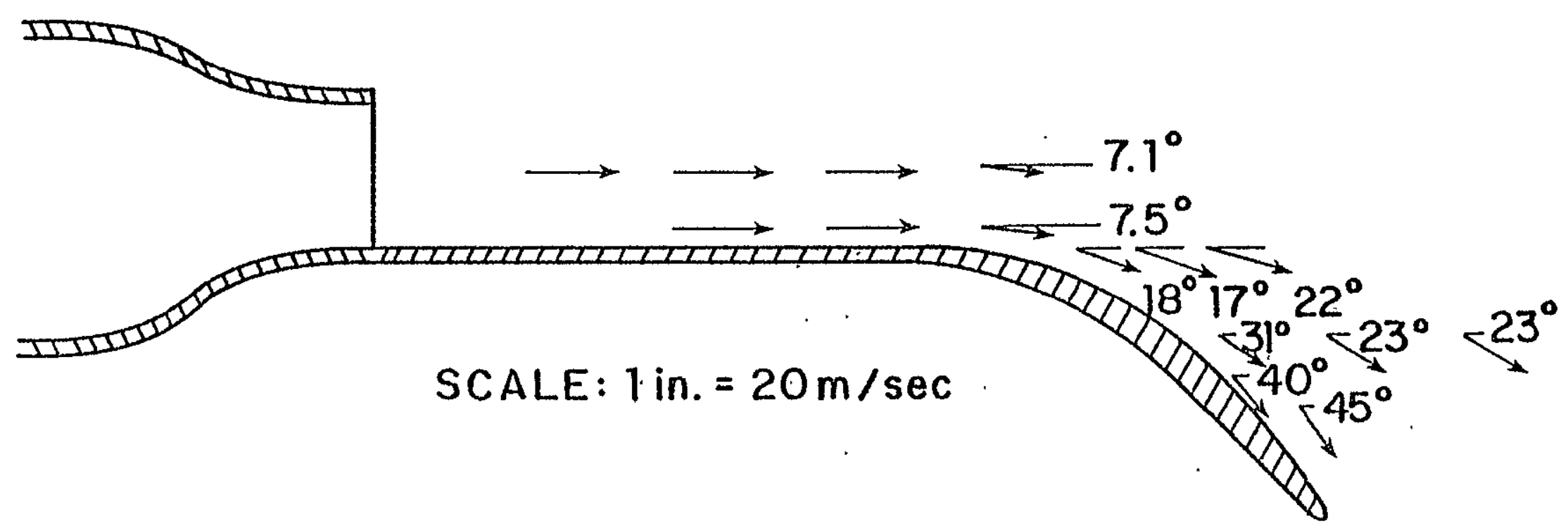

Figure 14 Vectorial Diagram of Flow Over Flap

$$
\left(\lambda_{j}=5.1\right)
$$


$W$ profile with the one at $z / 2 r_{0}=0.5$ yields the observation that the jet seems to be rotating or rolling up as it spreads out over the surface of the flap. While at $z / 2 r_{0}=0.5$, the maximum value of $W /\left(U_{0}-U_{F S}\right)$ is obtained at the centerline of the profile. For $z / 2 r_{0}=.185$, this velocity ratio remains small and constant in the. central region of the velocity field (i.e. $|y| / 2 r_{0}=0$ ) and reaches a maximum at approximately $|y| / 2 r_{0}=.90$. Hence, here an indication of the complexity of the three dimensional jet as it negotiates the curved wall surface clearly exists.

Mèan velocities in the lateral direction, $\nabla$, are measured and are also presented in Figure 15. Recal1 that the velocity measurements made in this report are conditionally sampled and thus the $V$ velocities are somewhat larger than might be otherwise expected for unconditional measurements. The mean lateral velocities are determined for the unconfined coflowing jet, the flow over the plate and the flow over the flap for the same downstream location $\left(x / 2 r_{0}=4\right.$ and $\left.z / 2 x_{0}=0.5\right)$ relative to the exit plane of the nozzle. While the maximum lateral velocities for both the unconfined flow and flow over the plate are comparable in magnitude, this is not the case for the flow over the flap. In fact, the maximum value of the ratio $\mathrm{V} /\left(\mathrm{U}_{\circ}^{\prime}-\mathrm{U}_{\mathrm{FS}}\right)$ has approximately doubled the maximum 


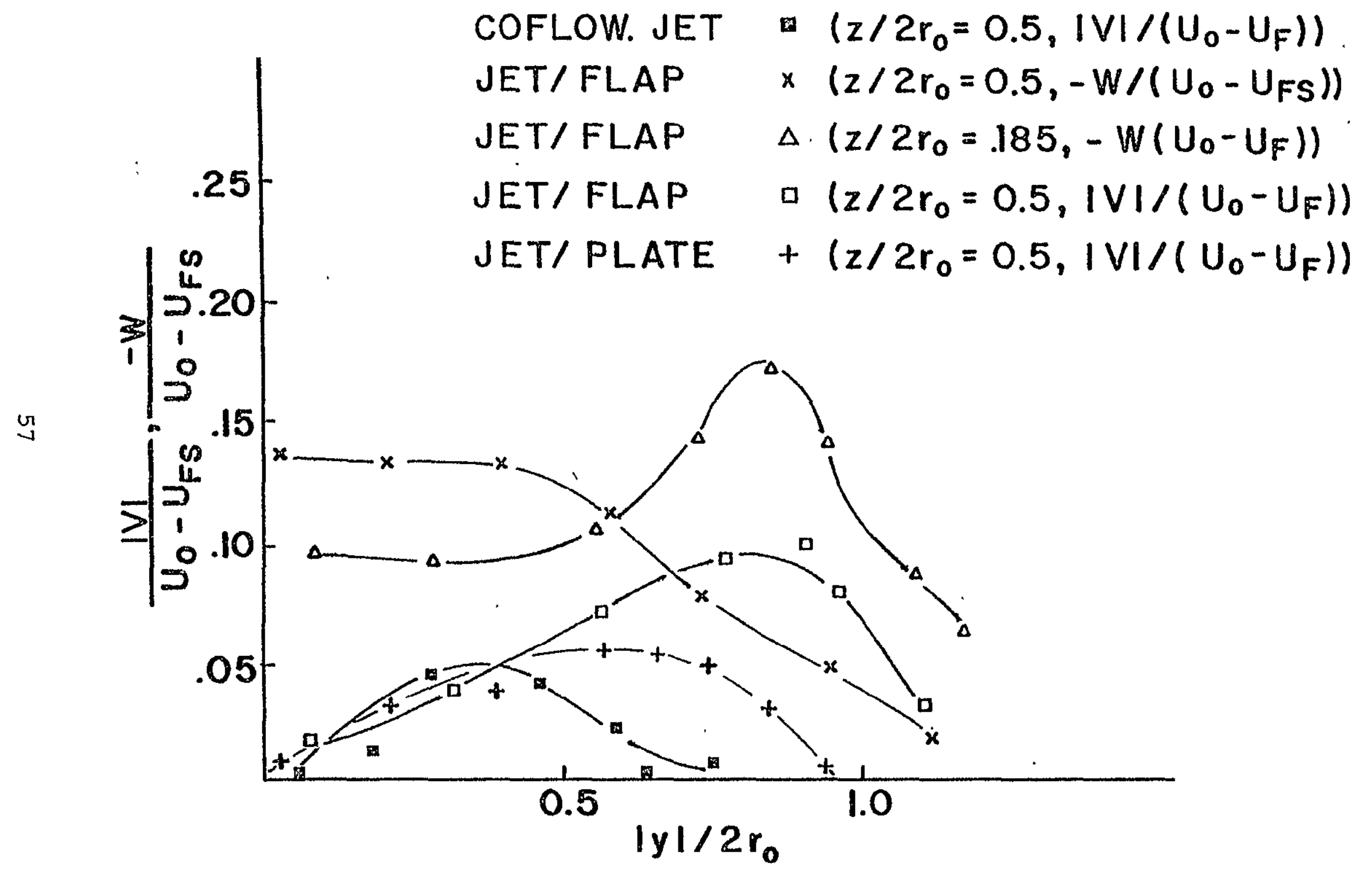

Figure 15 Vertical and Lateral Mean Velocity Profiles for Flap, $x / 2 x_{0}=4$ $\left(\lambda_{j}=5.1\right)$ 
value found for the other two flow configurations.

- A comparison of the mean velocity profile for the flow over the plate and the flow over the flap to the theoretically predicted classical wall jet profile is presented in Figure 16. The velocity profiles in the center plane $\left(|y| / 2 x_{0}=0\right)$ are tested for the fully developed classical wall jet type similarity by plotting $\left(U-U_{F S}\right) /\left(U_{\max }-U_{F S}\right)$ versus $z / z^{\frac{1}{2}}$. Clearly for the. data presented here, the "top hat" profile of the flow leaving the nozzle is still apparent. For any particular profile, Umax and the length scale $z^{\frac{1}{2}}$ are obtained from the smooth curve drawn through the experimental points. The length scale, $z \frac{1}{2}$, is the vertical distance from the wall surface to the point where $\left(U-U_{F S}\right)=0.5$ (Umax $-U_{F S}$ ) and dU/dy is negative.

The data presented in Figure 16 corresponds to three different downstream locations $\left(x / 2 r_{0}=3.76,4.0\right.$, and 4.24). Focussing initially on the mean velocity profile for the flow over the plate, it is clear that these plots are quite different in shape from the classical wall jet model. This is not surprising for it has been theorized that the wall jet actually is slower than the free jet in achieving similarity. Rajaratnam et. al. (31) suggested that similarity will not be achieved until approximately ten to fifteen diameters downstream. 


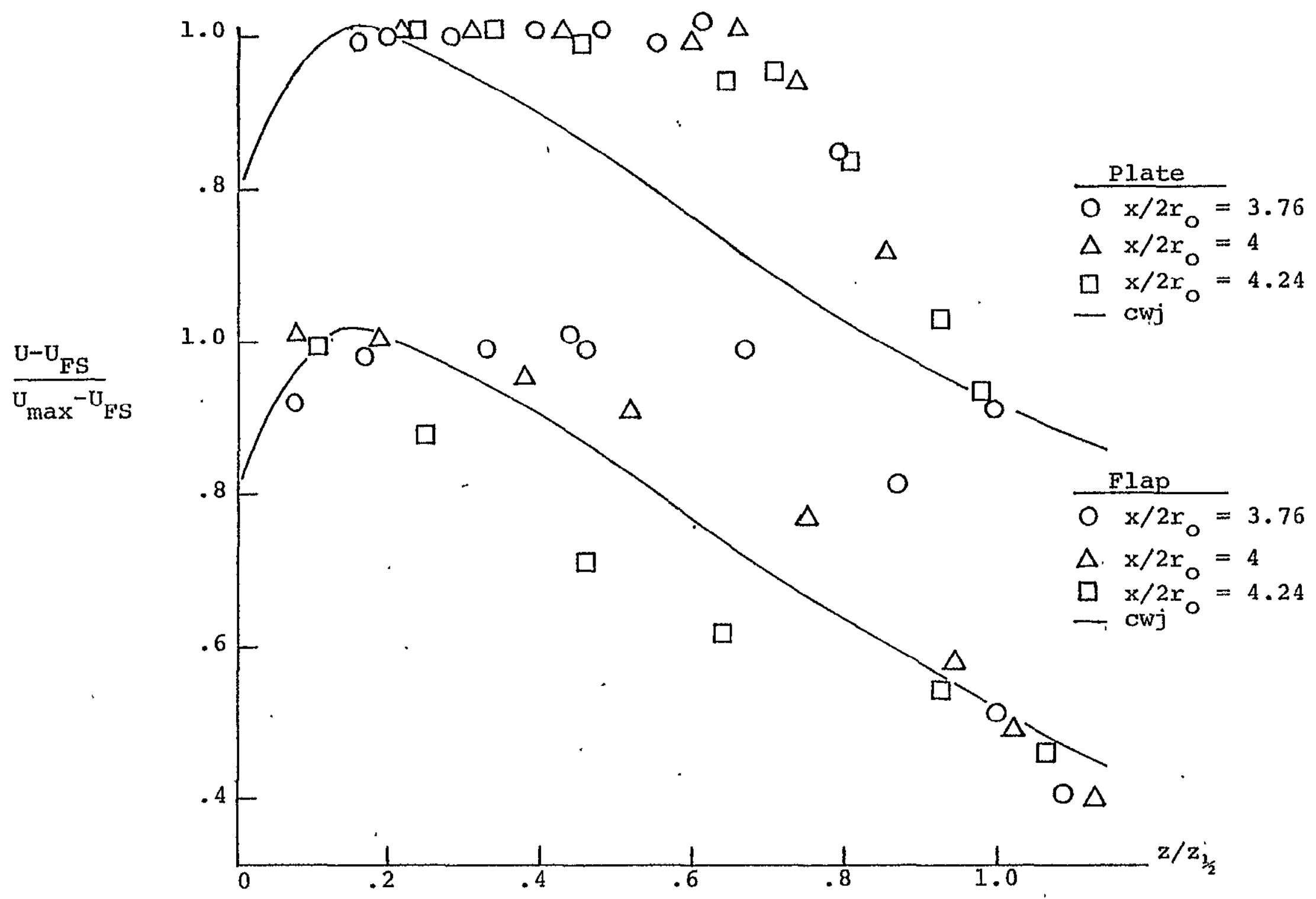

Figure 16 Comparisons to Classical Wall Jet (cwj), $|y| / 2 r_{0}=0$

$\left(\lambda_{j}=5.1\right)$ 


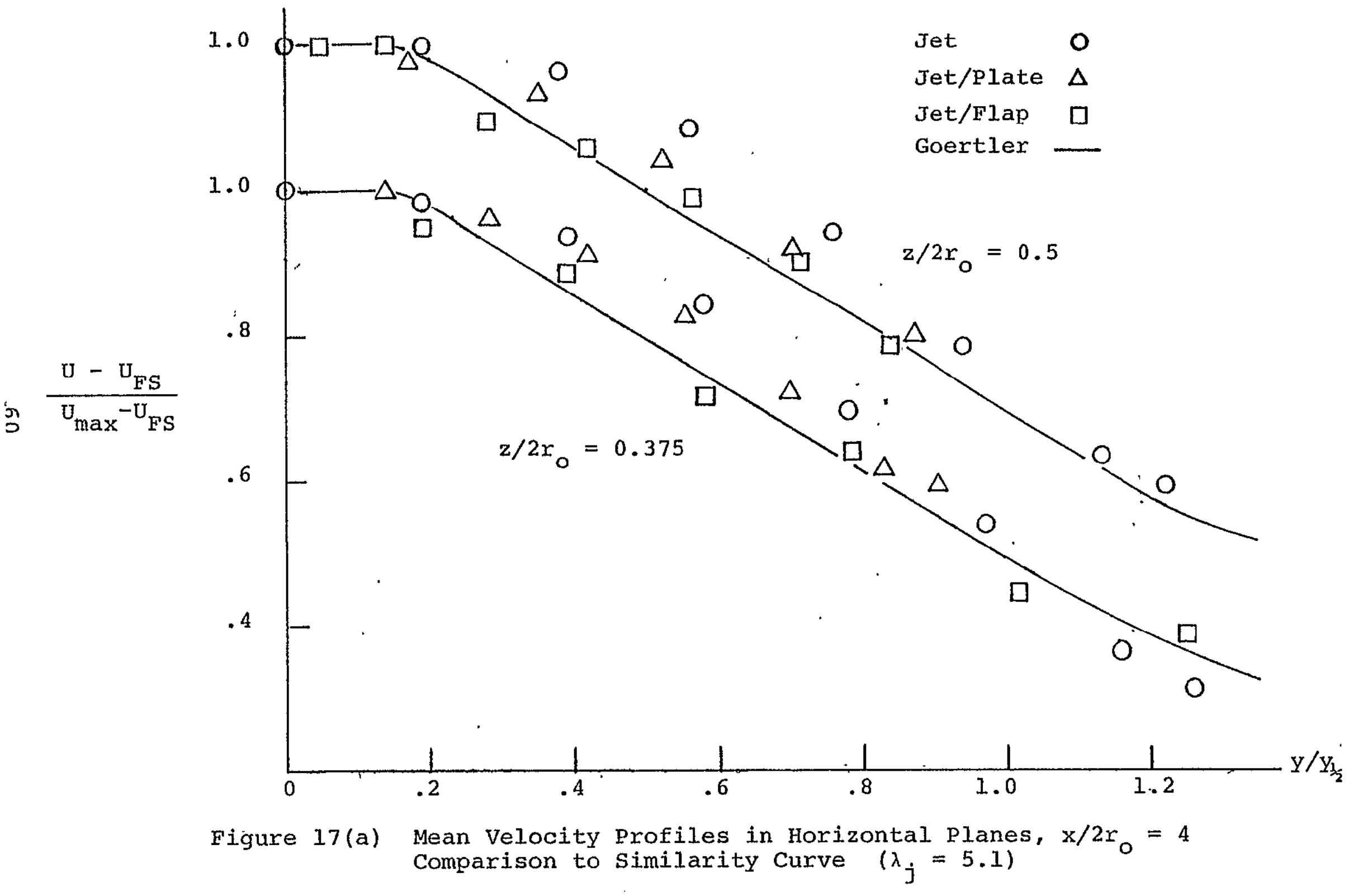




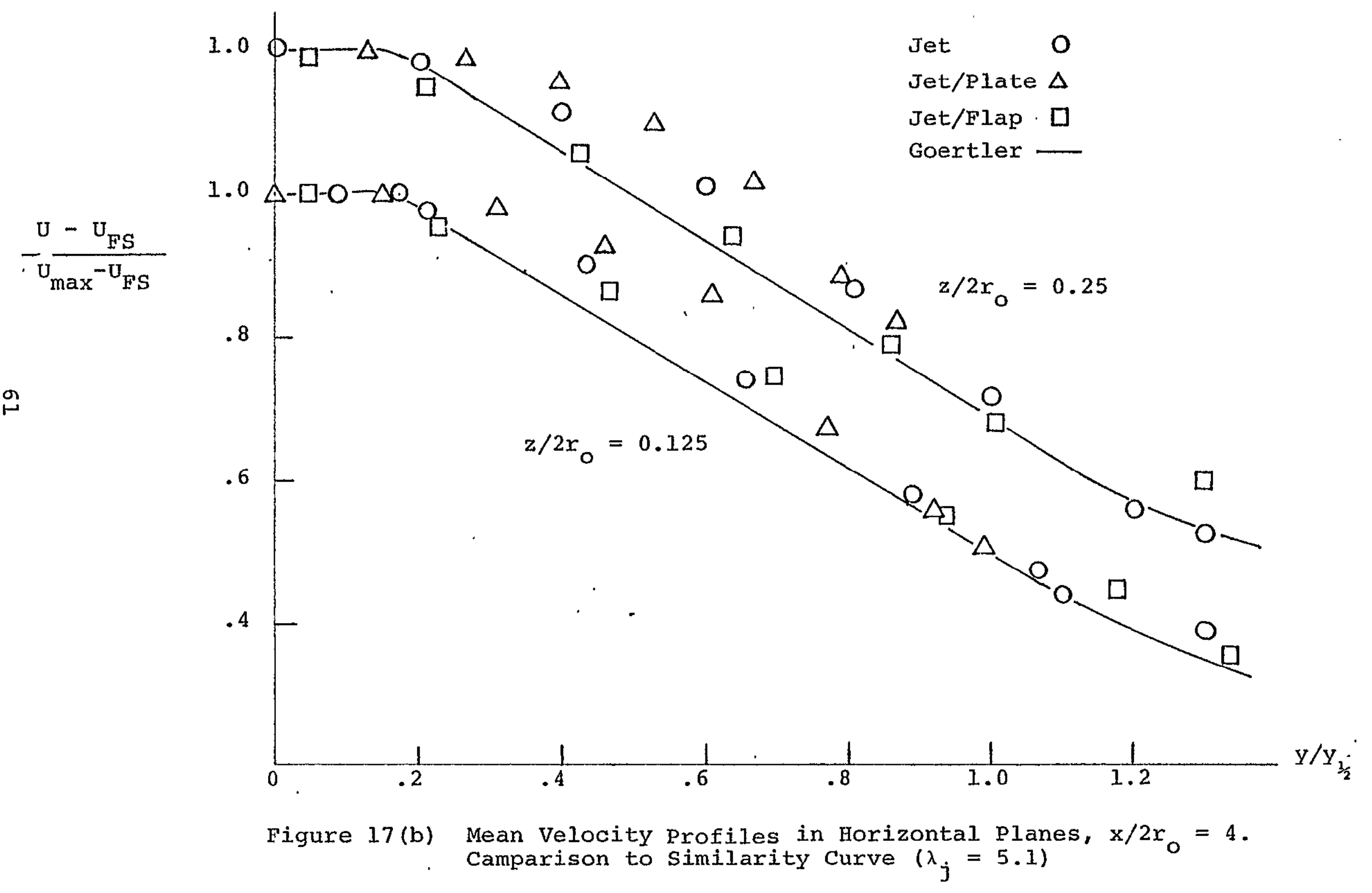


Turning next to a discussion of the flow over the flap at $x / 2 x_{0}=3.76$, the mean velocity profile is comparable to the profile for the plate. However at $x / 2 r_{0}=$ 4.0, the mean velocities ploted are close to the theoretical classical wall jet curve. After the flow has passed the actual curved portion of the flap $\left(x / 2 x_{0}=4.24\right)$, the closeness to theory is no longer present. In contrast to the flow over the plate, however, the mean velocity profile does lose its "top hat" or flat characteristic much more rapidly. Once again evidence is seen of the earlier breakup of the potential core region of the turbulent jet as it flows over the flap.

To check similarity of the mean velocity profiles in the horizontal planes at any height, $z / 2 \mathrm{r}_{0}$, from the wall, (U-UFS: $/\left(U_{\max }-U_{F S}\right)$ is plotted versus $y / Y_{I / 2}$ in Figure 17. The quantity $y_{1 / 2}$ is the length scale in the $y$ or transverse direction and is equal to the lateral displacement from the centerline for which $\left(U-U_{F S}\right)=$ $0.5\left(U_{\max }-U_{F S}\right)$.

The mean velocity profiles in the horizontal planes for the unconfined coflowing jet, the flow over the flap and the flow over the plate at a given $z$ location are compared to the curve predicted by Goertler ${ }^{(28)}$ for a free circular jet. The profiles for all three configurations are closer to the theoretical curve in the horizontal 
plane than is the case for classical wall jet comparison discussed previously. The mean velocity profile for the flap configuration is the closest to the similarity curve while the unconfined jet has the largest discrepancies for the vertical heights $z / 2 r_{0}=0.5$ and $z / 2 r_{0}=0.375$. However, closer to the surface it is the flow over the flap which deviates the most from Goertler's curve. From this discussion, it is apparent that the flow over the plate tends toward the fully developed turbulent shape in horizontal planes faster than in the vertical plane. Also, the flap seems most effective in transforming the flat velocity profile characteristic of a circular nozzle. With respect to the unconfined coflowing jet the mean velocity profiles are closer to the fully developed turbulent shape in the horizontal planes at $z / 2 x_{0}=0.125$ and 0.25 than is the case at $z / 2 x_{0}=$ 0.375 and 0.50 .

Section 4.1 Turbulent Intensities

Turbulent intensity is the ratio of the root mean square of the turbulent velocity fluctuations to the mean velocity at some reference location. In the investigation, the turbulence level is non-dimensionalized by the excess centerline velocity at, the exit plane of the nozzle (i.e. $U_{0}-U_{F S}$. Mathematically, turbulent intensity in the $x$ direction. is defined as follows: 


$$
\text { Turb. Intensity }=\frac{u_{\text {rms }}}{U_{0}-U_{F S}}
$$

where

$$
\text { urms }=\left\{\frac{I}{T} f_{0}^{T} u^{2} d t\right\}^{\frac{1}{2}}
$$

It should also be noted that the turbulence intensities were corrected for ambiguity noise using the methods of Morton. (61) The ambiguity noise was found to be approximately $1.8 \%$.

The distributions of turbulent intensities in the longitudinal direction and in the horizontal plane 10cated at $z / 2 x_{0}=0.5$ are plotted versus lateral displacement (i.e. $\left.|y| / 2 r_{0}\right)$ from the centerline in Figure 18. This data is for $\lambda_{j}=5.1$. For the case of the unconfined, coflowing jet, the turbulent intensity increases from about $2.5 \%$ to $5 \%$ from $x / 2 r_{0}=2$ to $x / 2 r=4$. In contrast to this development is the more rapid increase in turbulent intensity when either the plate or flap are in place. At $x / 2 x_{0}=2$, the turbulent intensities are all close in magnitude near the centerline. The largest value in fact, is maintained by the flow over the plate. At $\mathrm{x} / 2 \mathrm{r}_{\circ}=4$, however, the highest turbulent intensity is maintained by the flow over the flap. In fact irms/(U⿺ $\left.U_{0}-U_{F S}\right)$ is approximately five times the value it possessed at $x / 2 r_{0}=2$. 


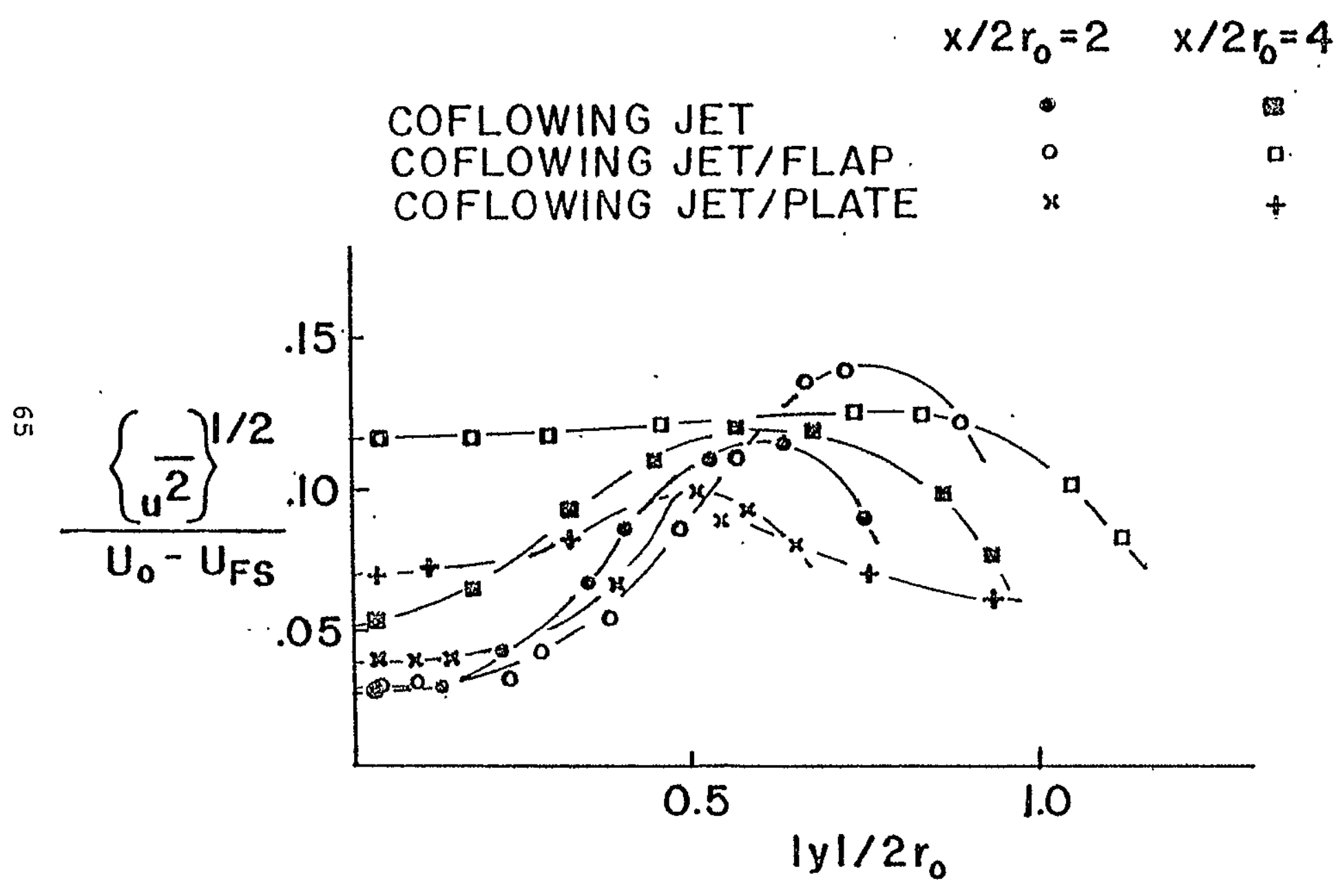

Figure 18 Longitudinal Turbulent Intensities Profiles, $2 / 2 r_{0}=0.5$ $\left(\lambda_{i}=5.1\right)$ 
Another important observation concerns the relative turbulent intensity value of the three flow configurations in the region $0.5 \leq|y| / 2 r_{0} \leq 1$. Whereas the flap seems to be amplifying the turbulence field in this region as well, the intensities for the plate flow are consistently lower than either, of the other two cases. The presence of the confining plate seems to be diminishing the turbulent fluctuations occuring in the outer regions of the Elow.

Turbulent intensities in the lateral direction, $\left(\bar{V}^{2}\right)^{1 / 2} /\left(U_{0}-U_{F S}\right)$, are shown. in Figures 19 and 20 . In Figure 19, a comparison of the intensities for the different flow configurations at the same downstream location $\left(x / 2 r_{0}=4\right.$ and $\left.z / 2 r_{0}=0.5\right)$ is presented. Notice that the turbulent intensity in the lateral direction is much higher in the shearing layer of the unconfined jet than in either of the other two configurations. In Figure $20,\left(\bar{V}^{2}\right) 1 / 2 /\left(U_{0}-U_{F S}\right)$ is presented for the flow over the flap. It indicates the change in the turbulence level as the flow reaches the curved portion of the flap structure.

The change of the ratios of the exit plane core velocity to the outer tunnel flow speed is seen to have a 


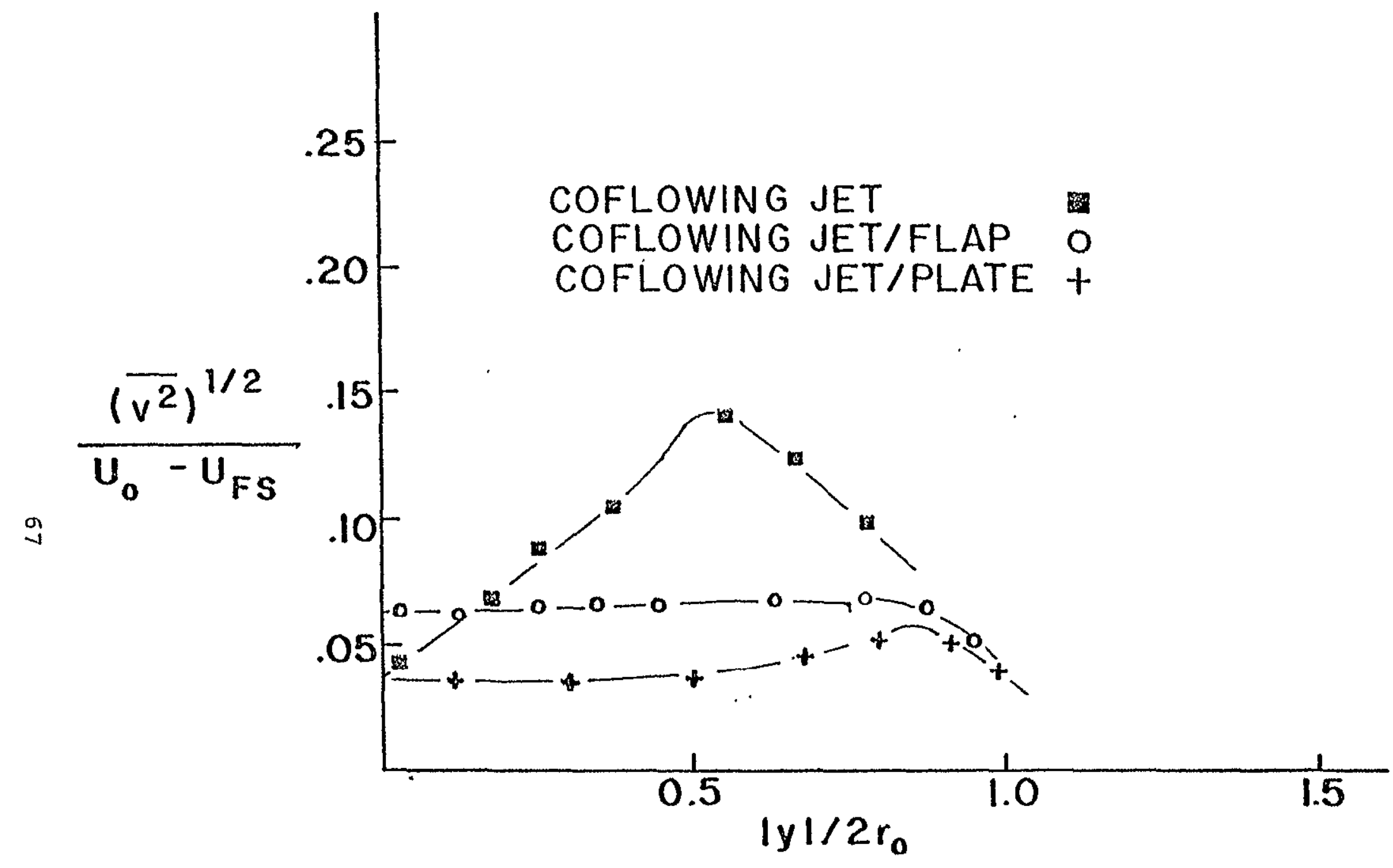

Figure 19 Lateral Turbulent Intensity Comparison, $x / 2 r_{0}=4$ and $2 / 2 r_{0}=0.5$ $\left(\lambda_{j}=5.1\right)$ 
ᄋి

\section{COFLOWING JET/FLAP}

$$
\begin{aligned}
& x / 2 r_{0}=2 \\
& x / 2 r_{0}=3 \\
& x / 2 r_{0}=4 \quad t .
\end{aligned}
$$

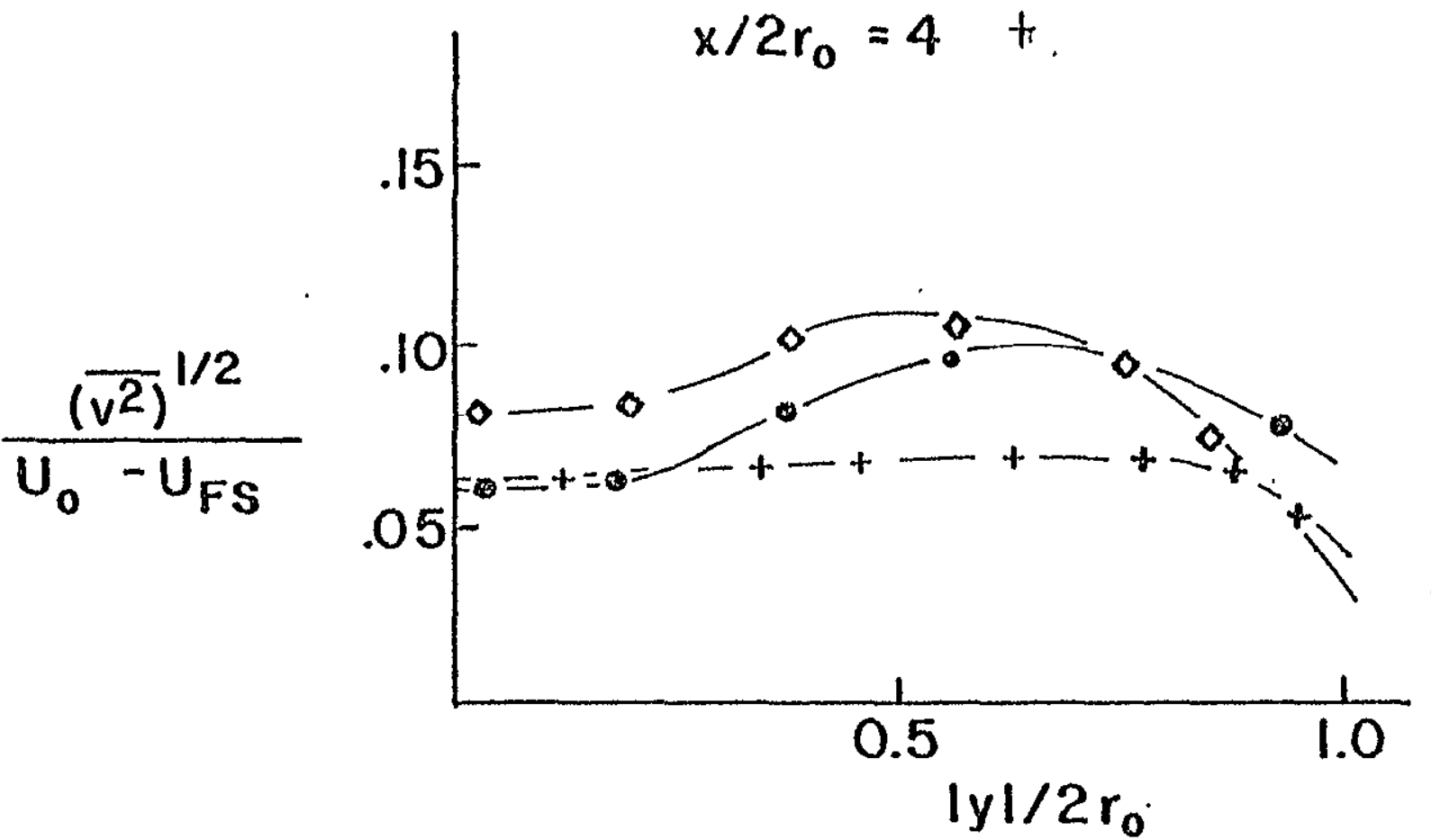

Figure 20 Development of Lateral Turbulent

Flow over Flap, $z / 2 r_{0}=0.5$

$\left(\lambda_{j}=5.1\right)$ 
significant effect on the decay of the centerline mean velocity. Next, the effect of varying $\lambda_{j}$ on the growth in the turbulent intensity at the centerline will be discussed. In Figure $21(a)$ and (b), the turbulent intensity at $y / 2 r_{0}=0$ and $z / 2 r_{0}=0.5$ is plotted versus downstream location, $x / 2 r_{0}$. For both $x_{j}=5.1$ and $\lambda_{j}=10.88$, the turbulence increases at about the same rate for both the unconfined coflowing jet and the flow over the plate. The magnitude is consistently higher. for the plate configuration. In fact, $\lambda_{j}$ seems to have very little effect on the experimental data.

For the flow over the flap, however, the value of the parameter, $\lambda_{j}$, is of considerable importance. In the case with $\lambda_{j}=10.88$, the increase in the turbulence is at only a slighter faster rate than is the case for the other two configurations. However, with $\lambda_{j}=5.1$, the increase is much more rapid. This fact is quite consistent with the observations made concerning the decay of the mean velocity. At the location in the flow field where the jet is decelerated and widened at the most rapid rate, the turbulence is also amplified greatly giving another strong indication that the flap serves to quickly break up the potential core flow. 

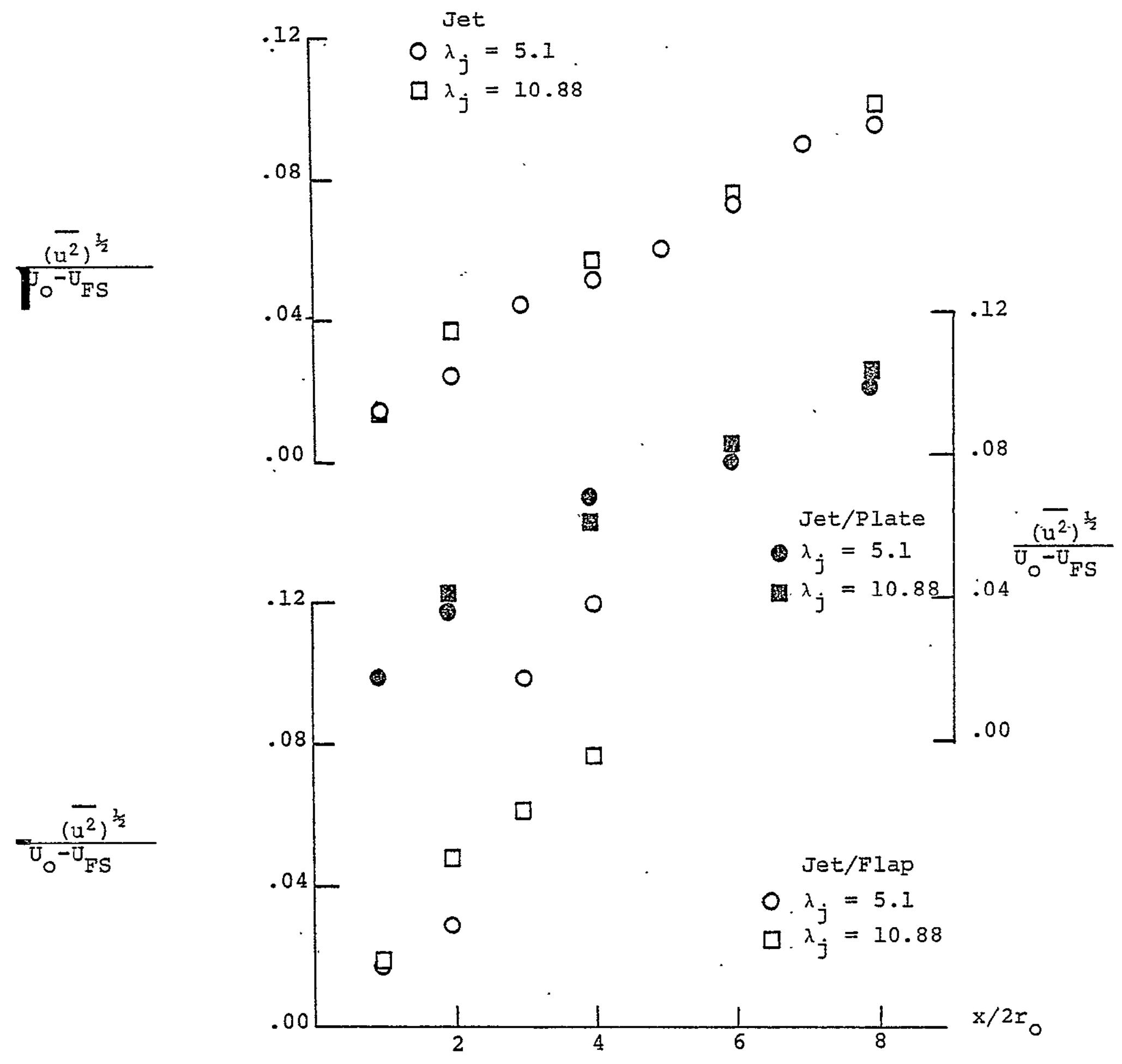

Figure 21 (a) and (b) Growth of Turbulent Intensity at Centerline 
Section 4.2 Longitudinal Autocorrelations

If $u=u(x, y, z, t)$ and $u^{\prime}=u(x+\Delta x, y ; z, t)$ are the longitudinal fluctuating velocites at different downstream locations $(x, x+\Delta x)$ but at the same lateral and vertical positions $(y, z)$ in the flow, then, at a given time, the correlation coefficient, $\mathrm{R}_{\mathrm{uu}}{ }^{\prime}$, is defined by:

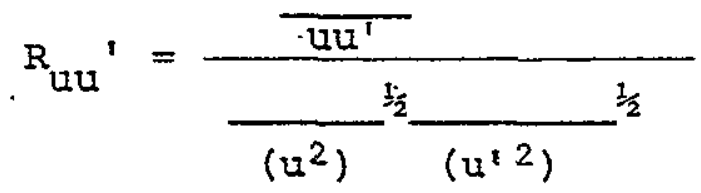

The autocorrelation coefficient, $R$, is given by:

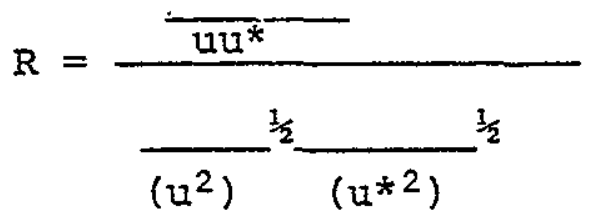

where $u^{*}=u(x, y, z, t+\Delta t)$. Autocorrelations can be converted to longitudinal correlations by using Taylor's hypothesis. (1) The basic tenet of this hypothesis is as follows :

$$
\frac{d}{d x}(B)=\frac{d}{d t}
$$

where $B$ is a statistical property. The assumption that is required is that the turbulent intensity not be too large, and that the flow be stationary. As was the case for the discussions of the mean velo- 
city and turbulent intensity, focus will be placed on both determining the effects of the two surfaces on the turbulent jet and also to examine the importance of the value of the parameter, $\lambda_{j}$.

- Initially, recall the vortex street model for a turbulent jet. In this model, it is theorized that vortices are shed from the lip of the nozzle and move downstream. In the potential core region of the flow, the turbulence level is very low. Thus, it is possible to detect the relatively small effects of the fluctuating pressure field on the turbulent velocity. The resultant fluctuating velocities are due to the passing downstream of vortices being shed at the lip of the nozzle. These shedding vortices would give the autocorrelation curve a damped sinusoidal shape. The spacing of the peaks of the curve would correspond to the dominant frequency in the flow field.

Consider the autocorrelations of the axial velocity fluctuations taken at the locations $|y| / 2 x_{0}=0$ and $|y| / 2 r_{0}=0.5$ and at the vertical height, $2 / 2 r_{0}=0.5$. The autocorrelations are determined in all three flow field at both two and four diameters downstream of the exit plane of the nozzle. The lateral positions $|y| \cdot / 2 r_{0}=$ 0 and $|y| / 2 x_{0}=0.5$ correspond to the centerline of the flow field and the lip of the nozzle. In Figure 22, 


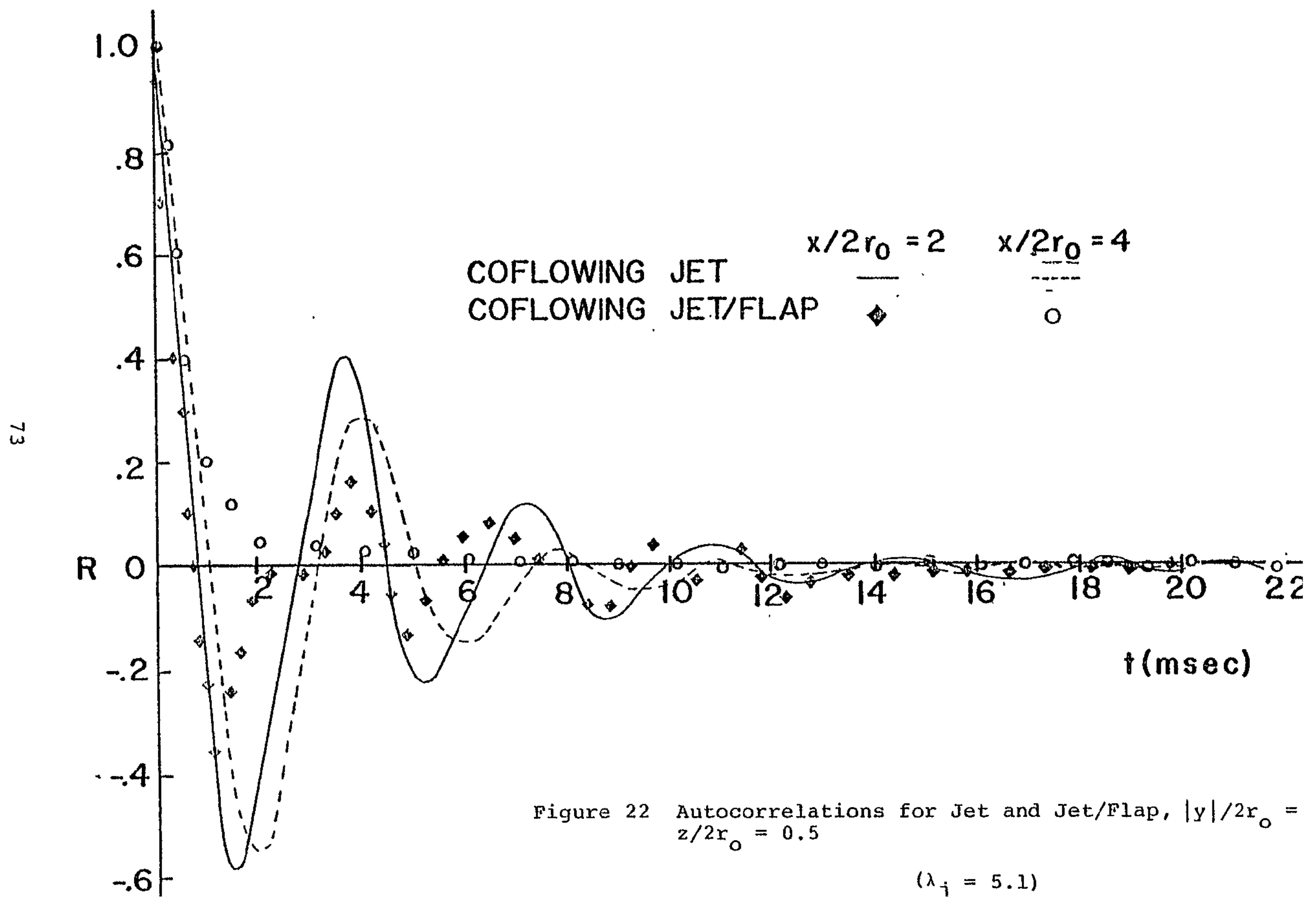


curves are presented for the unconfined, coflowing jet and the flow over the flap. These were taken at the centerline for both flow fields. Notice that for the unconfined jet, the autocorrelation coefficient has a damped sinusoidal behavior at both two and four diameters downstream. This is characteristic of the autocorrelation coefficient in the potential core region of the jet. Also, there is a slight change in the delay time which corresponds to a change in the dominant frequency of the flow.

With the flap placed in the flow, the sinusoidal nature of the autocorrelation curve is first reduced at $x / 2 x_{0}=2$ and then eliminated completely at $x / 2 x_{0}=4$. This is consistent with the rapid increase which was noted previousiy in the turbulent intensities in what was the potential core region. As is the case in the shearing layer region of the flow, the increase in the turbulence level masks out the relatively smaller velocity fluctuations. which are caused by the passing of vortices.

Still limiting the scope of the present discussion to the effects of the flap on the turbulent jet, autocorrelation coefficients are presented for the lateral location $|y| / 2 r_{0}=0.5$ in Figure 23. Here, the measurements are made in the mixing region of both flow 


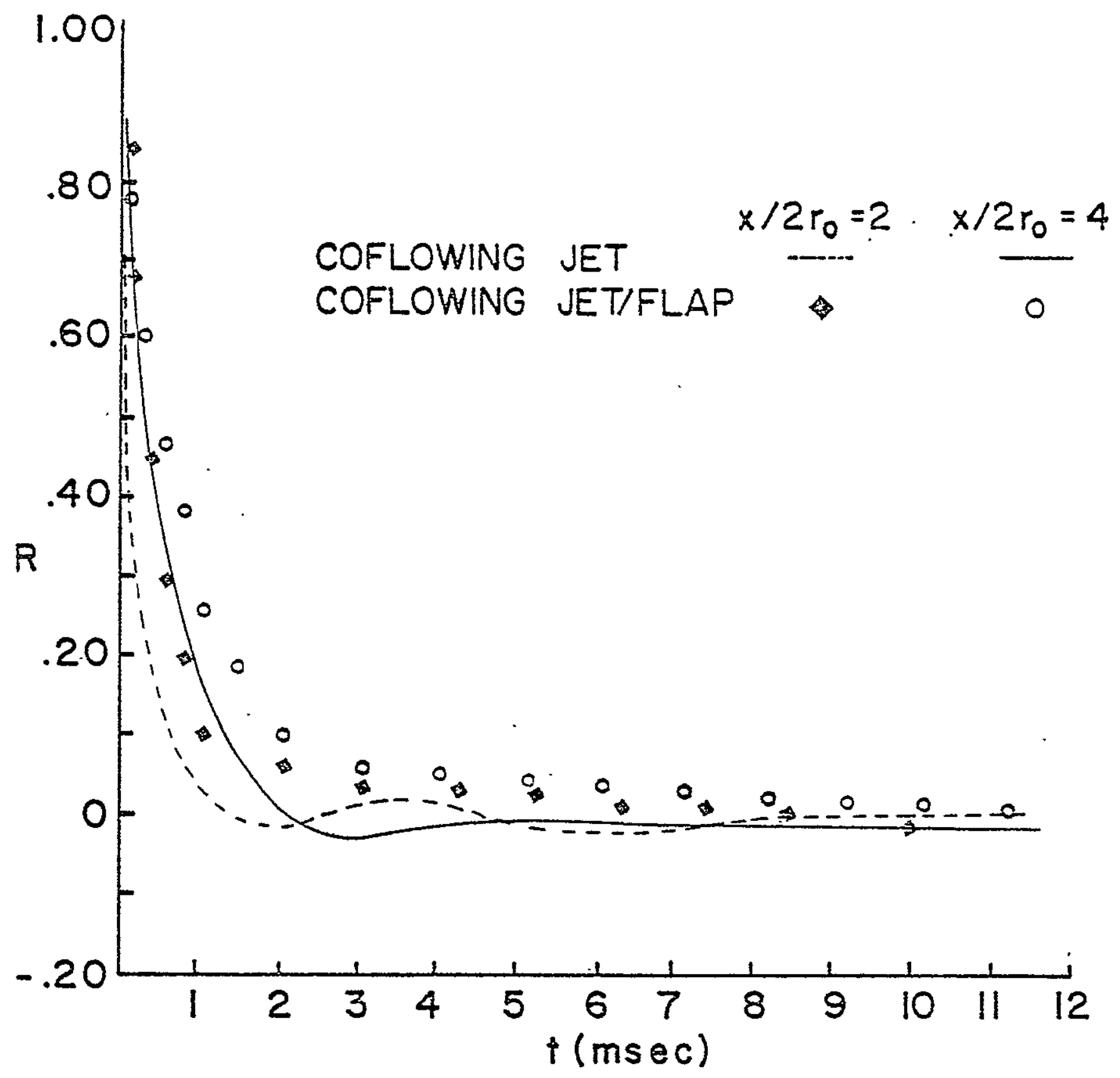

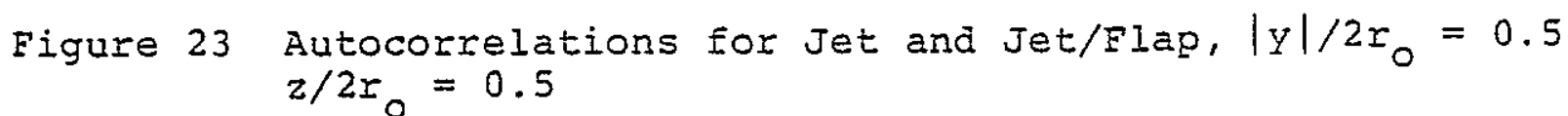

$$
\left(\lambda_{j}=5.1\right)
$$


configurations. Notice that there is little difference in the autocorrelations curves. The very high turbulence levels serve to completely mask out the velocity fluctuations due to the passing vortices and eliminate the damped sinusoidal nature of the autocorrelation curves measured in the potential core. Turning next.to a discussion of the effects of the plate on the turbulent jet, longitudinal autocorrelations are presented in Figure 24. At the centerline location, the autocorrelation looks quite similar to those determined for the unconfined case. In fact, for the case of the flow over the plate, the peaks are slightly more pronounced though the dominant frequency is the same at two diameters downstream. At four diameter downstream, the increase in the turbulence level for the flow over the plate seems to be slightly more effective in masking out the pressure fluctuations though not nearly as effective as the flap. In the mixing region as has been discussed before, the autocorrelation curves are similar in shape for all three flow configurations.

As is the case in other measurements reported, determining the effects of varying the outer tunnel flow speed on the various autocorrelation coefficient curves is also desired. To this end, the measurements of the autocorrelations are presented for the case when $\lambda_{j}=10.88$. 


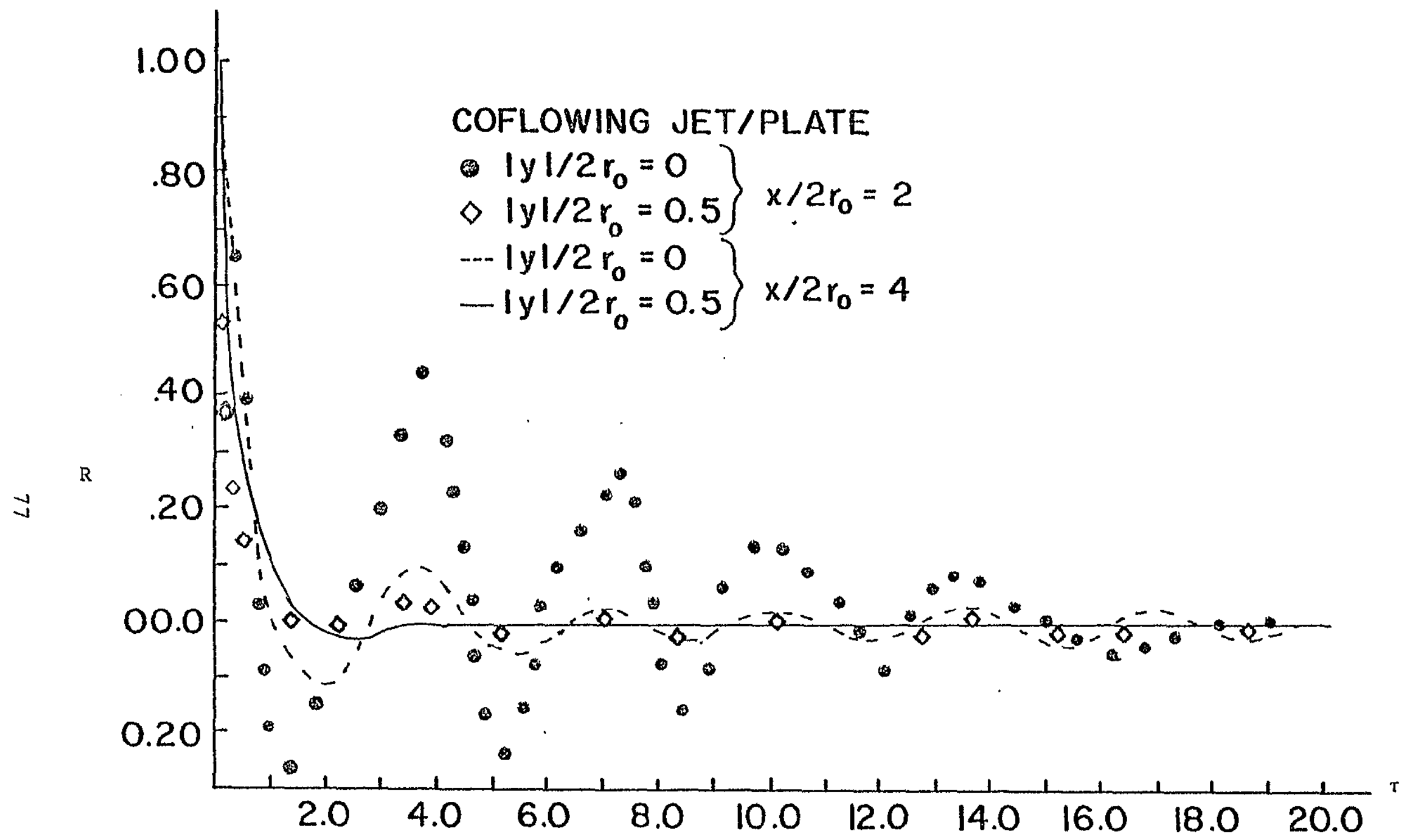

Figure 24 Autocorrelations for Jet/plate, $z / 2 r_{0}=0.5$

$\left(\lambda_{j}=5.1\right)$ 
The format used for the following data is as follows: two sets of curves are shown for each of the three flow configurations, one set corresponding to the centerline location $\left(|y| \cdot / 2 x_{0}=0\right)$, the second for the lateral 10cation directly downstream from the lip of the nozzle $\left(|y| / 2 r_{0}=0.5\right)$. The data is presented in this somewhat different manner than, was the case before in order that the change in the shape of the autocorrelation curves as the flow progresses downstream be more easily discerned.

In Figures 25 and. 26, autocorrelation coefficient curves for the unconfined, coflowing jet are presented. The characteristic damped sinusoidal nature is apparent at two and four diameters downstream at the centerline, though there is a slight shift in the dominant frequency and a small increase in the damping at $x / 2 r_{0}=4$. At eight diameters downstream, the autocorrelation curve has a typical shape for a fully developed turbulent flow. Out in the shearing region corresponding to the lip of the nozzle, the autocorrelation coeficients curves are devoid of any dominant frequency and remain essentially unchanged as the flow proceeds downstream.

Turning next to the case of the turbulent jet flowing over the plate, the resultant autocorrelation coefficient curves are shown in Figures 27 and 28 . Here, as is the 


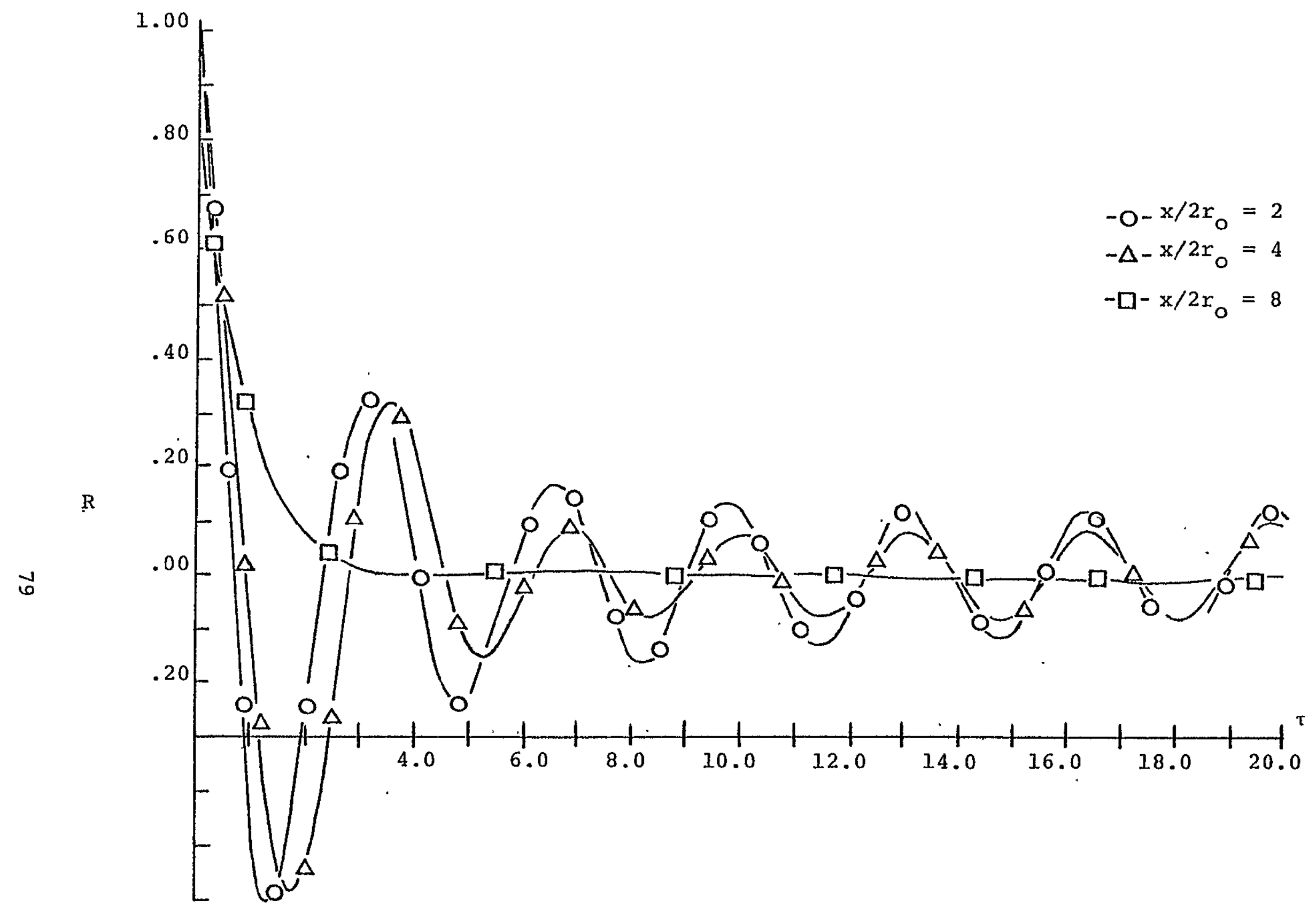
Figure 25 Autocorrelations for Jet, $|y| / 2 x_{0}=0.5, z / 2 x_{0}=0.5$
$\left(\lambda_{j}=5.1\right)$ 


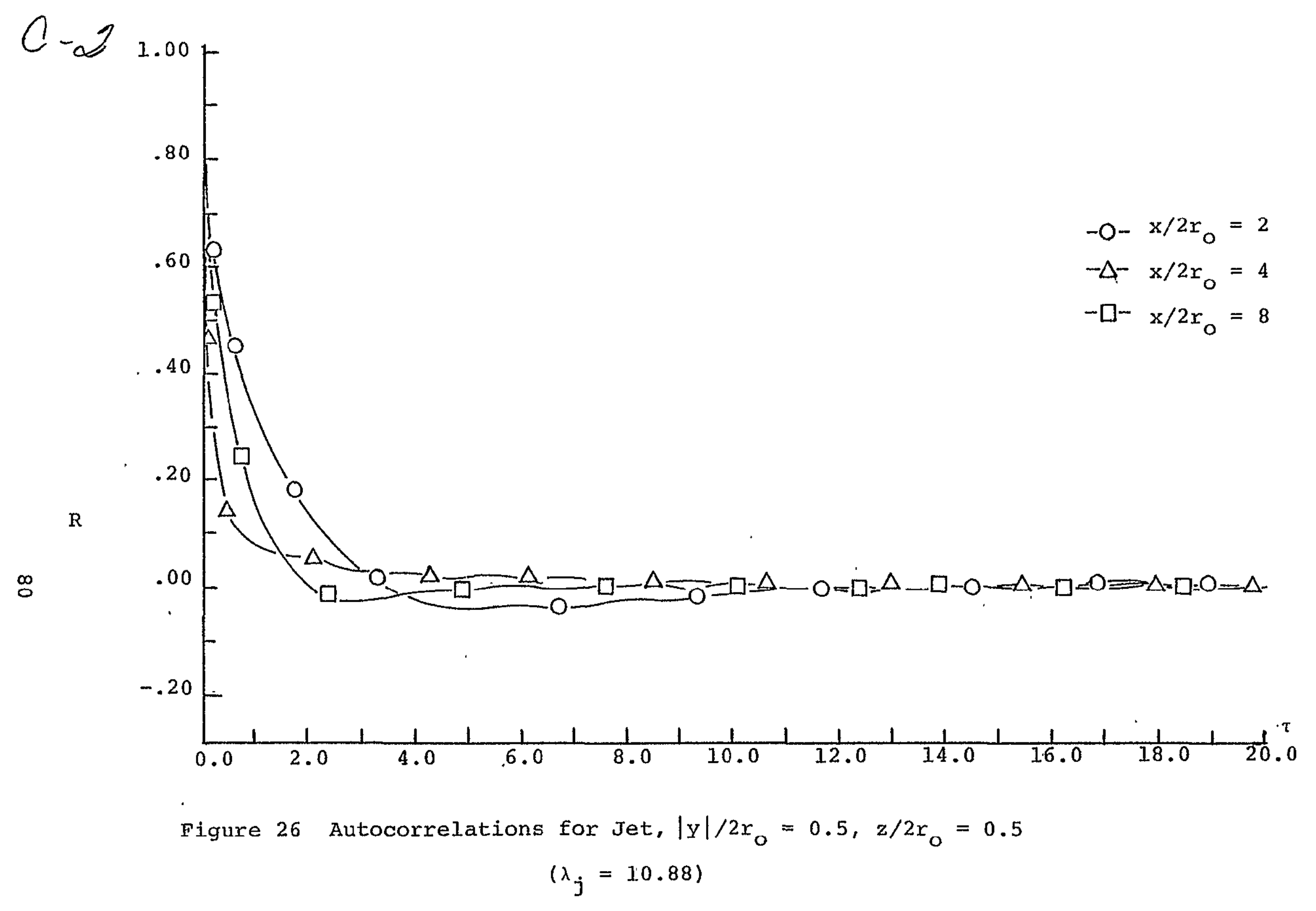




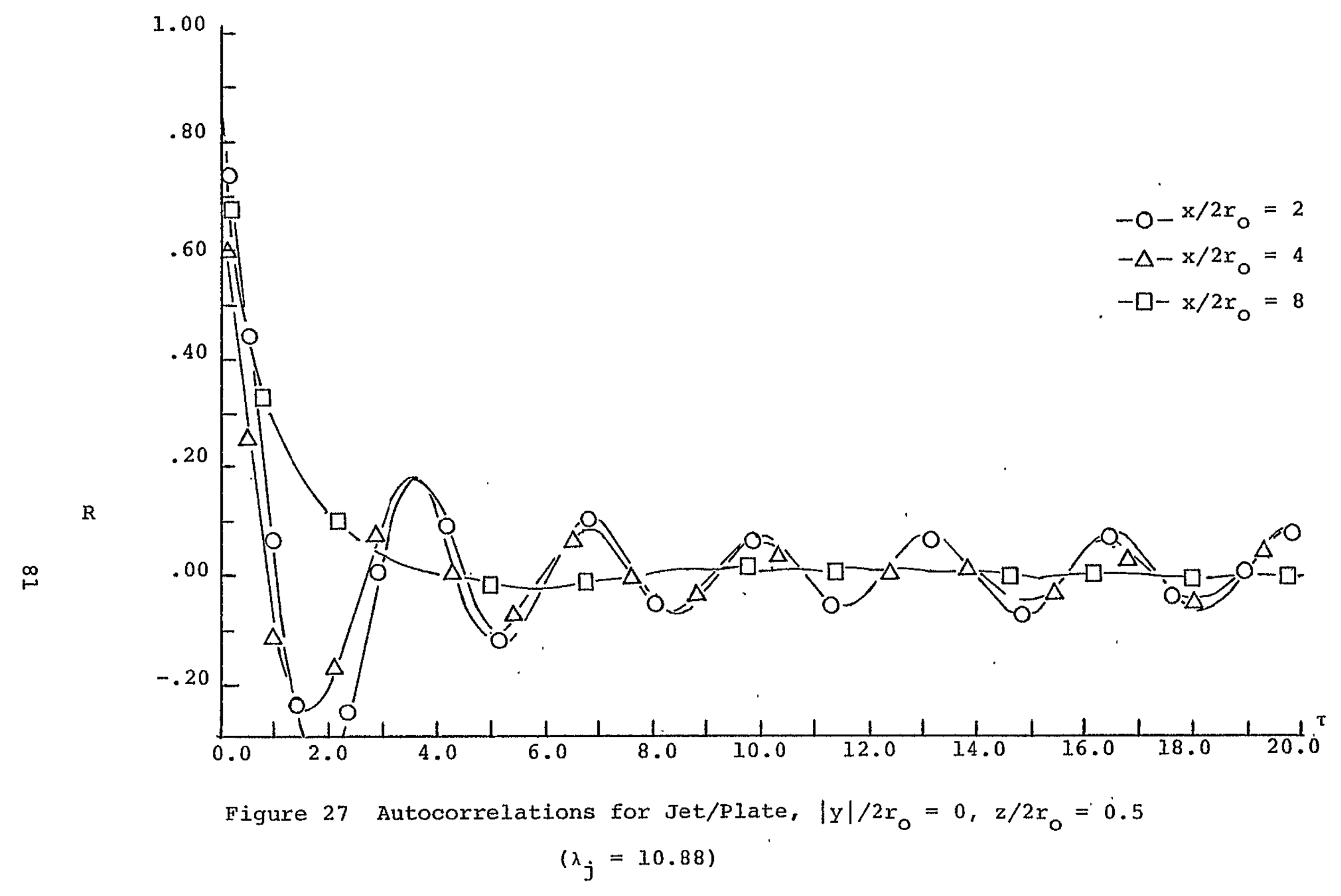




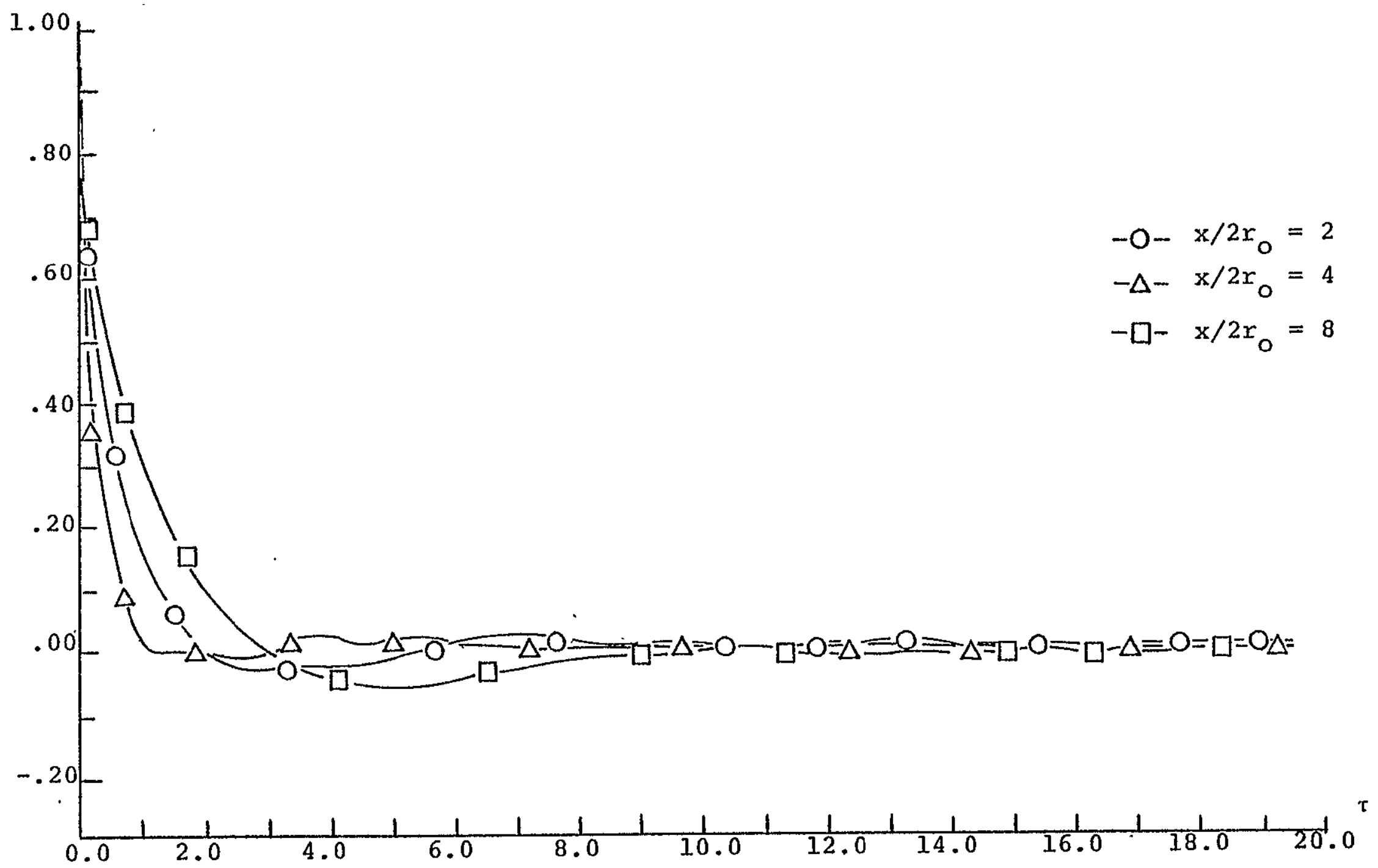

Figure 28 Autocorrelations for Jet/Plate, $|\mathrm{Y}| / 2 r_{0}=0, z / 2 r_{0}=0.5$

$$
\left(\lambda_{j}=10.88\right)
$$


case for the unconfined jet, the curves presented for the centerline location have the damped sinusoidal nature at both two and four diameters downstream of the nozzle. Also, there exists a slight shift in the dominant frequency and an increase in the damping. At eight diameters, the turbulent velocity field again seems to have "Iost" its structure. It is instructive to compare the autocorrelation curves measured for both values of $\lambda_{j}$. At the centerline, the dominant frequency in the autocorrelation coefficient curves is slightly greater when $\lambda_{j}=10.88$, both at $x / 2 r_{0}=2$ and $x / 2 r_{0}=4$. Also, the magnitude of the peaks in the curves are consistently larger at large delay times when $\lambda_{j}=10.88$. Next, consider the comparison between the results for the flow over the plate to the unconfined coflowing jet when $\lambda_{j}=10.88$. The dominant frequencies are shifted to a slightly higher value and the magnitudes of the individual peaks are damped to a greater extent with the plate in the place. This is exactly the same observations that are made when $\lambda_{j}$ is equal to 5.I. In the shearing region downstream from the lip of nozzle, the autocorrelation coefficient curves are more typically turbulent, in nature and are quite similar to the data obtained when $\lambda_{j}=5.1$. 
Finally, autocorrelation coefficient curves are shown for the flow of the turbulent jet over the flap in Figure 29 and 30 . Here, something quite interesting and maybe unexpected is occuring. At two diameters downstream from the exit plane, the curve again has a damped sinusoidal type behavior. However, $x / 2 r_{0}=4$, the flow cannot be characterized by a dominant frequency and behaves as if it is fully turbulent. This more rapid break-up of the potential core with the flap in place was noted for the case when $\lambda_{j}=5.1$, but that similarity is somewhat surprising. Recall that the flow did not decelerate nor widen as quickly when $\lambda_{j}$ is set equal to 10.88. Also, the turbulent intensities at the centerline of the flow did not increase as rapidly. Yet, the autocorrelation coefficient curves indicates that the turbulent structure of the jet has been similarly diffused in both cases. Thus, there exists a strong indication that the structure of the turbulent velocity field is influenced to a far greater extent than the change in the time averaged mean velocities would indicate. Downstream from the lip of the nozzle, the curves again assume their customary shape. Here, a similarity exists between the cases when $\lambda_{j}$ equals 5.1 and also 10.88 . 


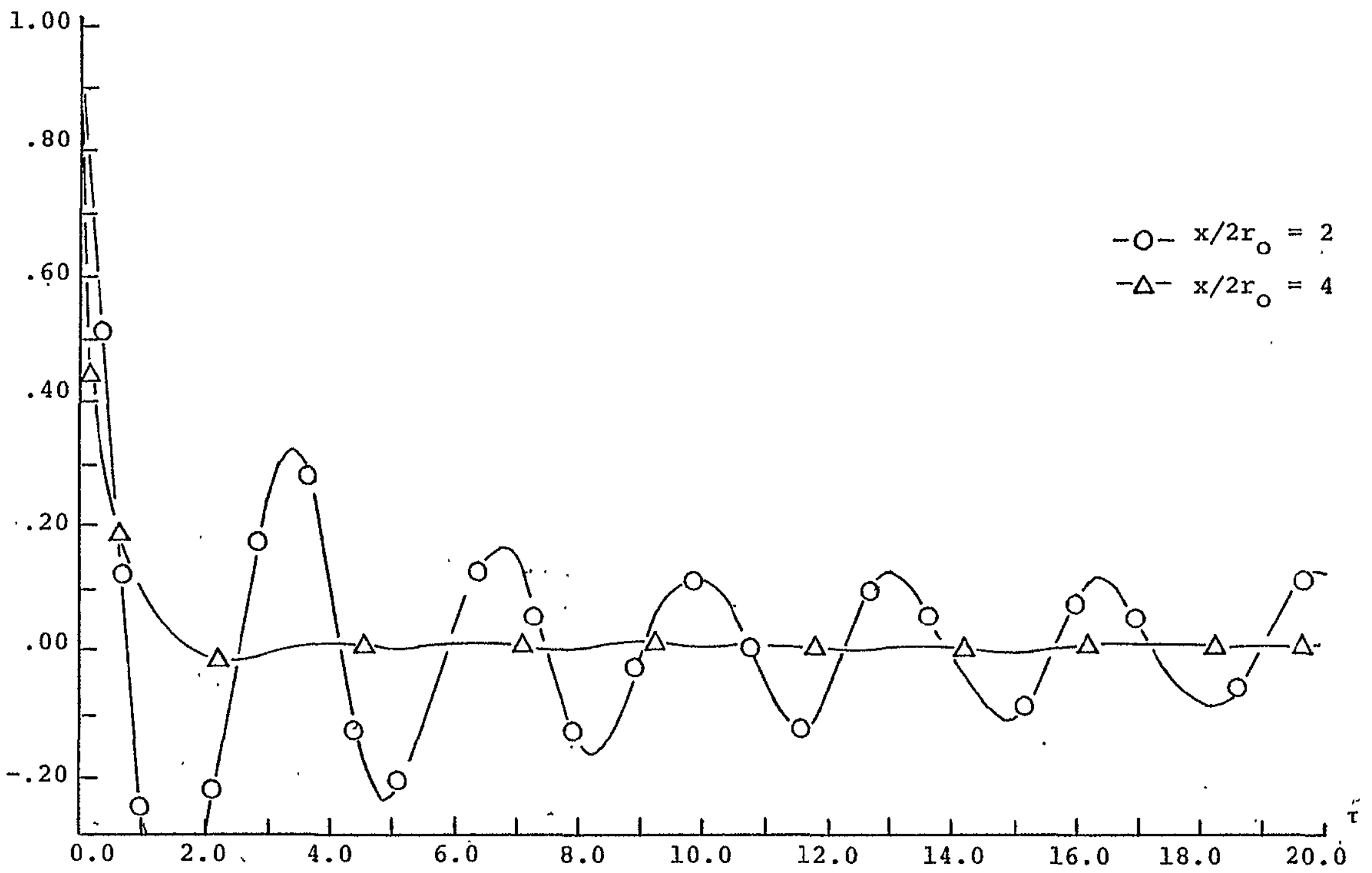

Figure 29 Autocorrelations for Jet/Flap, $|y| / 2 r_{0}=0, z / 2 r_{0}=0.5$

$$
\left(\lambda_{j}=10.88\right)
$$


Focussing on the comparison of the autocorrelation coefficient curves in the potential core for all three flow configurations when $\lambda_{j}$ equals 5.1 and when $\lambda_{j}$ equals 10.88, though the shapes are quite similar, there is one difference which should be noted. At the larger delay times, the peaks of the curves are somewhat larger in magnitude. Thus, for the same flow configurations, the damping at large delay times seems to be a function of the inner jet to outer flow velocities.

Section 4.3 Integral Scales

The longitudinal integral time scale in the $x$ direction is defined in terms of the autocorrelation coefficient as follows:

$$
T_{1}=\int_{0}^{t^{*}} u(t) u\left(t^{\prime}\right) d t^{\prime}
$$

or

$$
T_{1}=\int_{0}^{t *} R\left(t^{\prime}\right) d t^{\prime}
$$

where $t^{*}$ is the time at which the coefficient first reaches the value of zero. To obtain the length scale, the integral time scale is multiplied by the local mean velocity, again using the "frozen" turbulence approximation. (1)

$$
\text { Thus } \mathrm{L}_{1}=U \times \mathrm{T}_{1}
$$


Physically, the integral length scale is approximateIy the largest turbulent scale in the flow.

The growth of the integral length scale for the unconfined jet for both values of $\lambda_{j}$ is addressed in Figures $3 I(a)$ and $3 I(b)$.

Figures $3 I$ (a) deals with the development of the integral scale at the lateral location corresponding to the lip of the nozzle while Figure $31(b)$ is concerned with the centerline growth. At the lip of the nozzle, the integral length scale is found to grow linearly downstream as has been predicted by Iaurence The scale for the unconfined jet with $\lambda_{j}=5.1$ grows somewhat faster than is the case when $\lambda_{j}=10.88$. At the centerline of the flow field, the integral scale grows similarly for both values of $\lambda_{j}$. This is not too surprising since the velocity of the outer tunnel flow would not be expected to have a significant effect on the turbulence in this region for the first several diameters downstream.

For the flow over the flap, the development of the integral scale is quite different than is the case for the coflowing jet and is presented in Figure 32 . It seems possible that the values measured at both the centerline of the flow field and out at the lateral location of the lip can be plotted on one curve. Also, the value of 

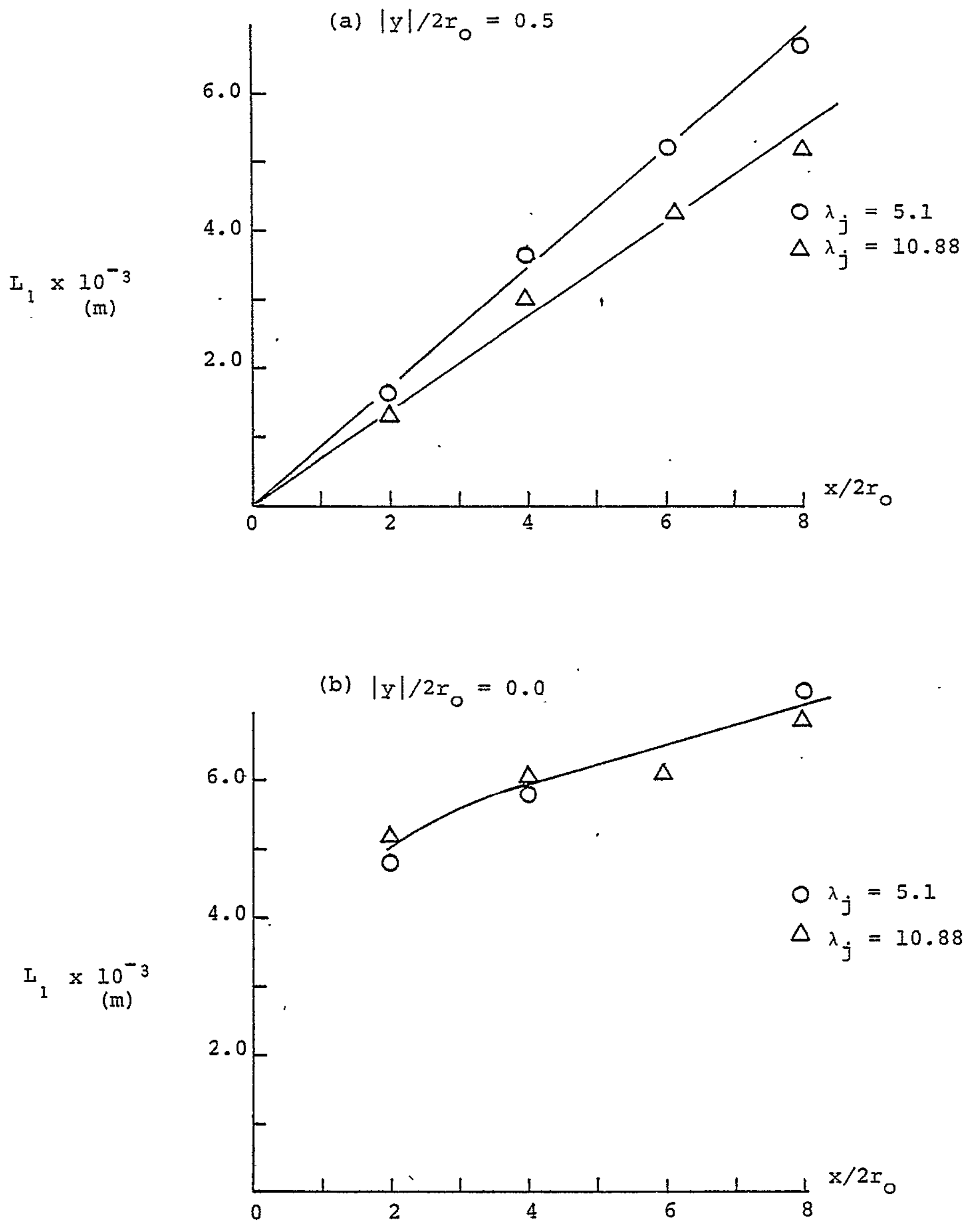

Figure 31 Development of Integral Scale for Jet, $z / 2 r_{0}=0.5$ 
$\lambda_{j}$ does not seem to influence the results. The rapid increase and then decrease of the values of the integral scales are notable in two respects. First, the large jump in the value of $I_{1}$ actually takes place before the flow has reached the curved portion of the flap. This is also true with respect to the steep decline. An understanding of this behavior lies in an examination of the definition of the integral length scale. First, since the flow field over the flap grows at the most rapid pace, one would expect the integral length scale, which is an estimate of the largest scale in the flow to reflect this increase. However, as the flow approaches the actual curved portion of the flap, there occurs a turning of the flow direction and also a rapid transforming of the "top hat" mean velocity profile into a profile more typical of a wall jet. 'Thus, the faster moving fluid particles are closer to the flap surface while out at $z / 2 r_{0}=0.5$, the fluid is moving considerably slower. This large decrease in the mean velocity would cause a decrease in the magnitude of the integral length scale if the integral time scale does not increase significantly since it is the product of integral time scale and the local mean velocity which are of importance. In fact, the integral time scale is nearly constant between $x / 2 r_{0}=2$ and $x / 2 r_{0}=4$. 
Secondly, as was noted previously, the integral scale is at best a very weak function of the value of $\lambda_{j}$. Though the presence of the flap amplifies the turbulence in the jet to a lesser extent when $\lambda_{j}$ is equal to 10.88 , the product of the local mean velocity and the local integral time scale seems independent of the outer tunnel speed. This, however, is difficult to determine precisely since the gradients of the integral scale are very large.

The growth of the integral scales for the flow over the plate is presented in Figures $33(a)$ and $33(b)$. For both $\lambda_{j}=5.1$ and $\lambda_{j}=10.88$, the integral. scale at the centerline is consistently larger in magnitude than it is at the lip of the nozzle. After roughly four diameters downstream from the exit plane, the growth rate for all four curves shown is approximately the same. In, the data presented here, the value of $\lambda_{j}$ does effect the magnitude of the integral scale for the first few diameters downstream. However, after that, virtually no influence can be ascertained.

\section{Section 4.4 Taylor Microscales}

Let $u^{\prime}$ be the fluctuation of the longitudinal velocity in the ambient flow, then the Taylor microscale, $\lambda_{T^{\prime}}$ of turbulence is determined from the following condition. 


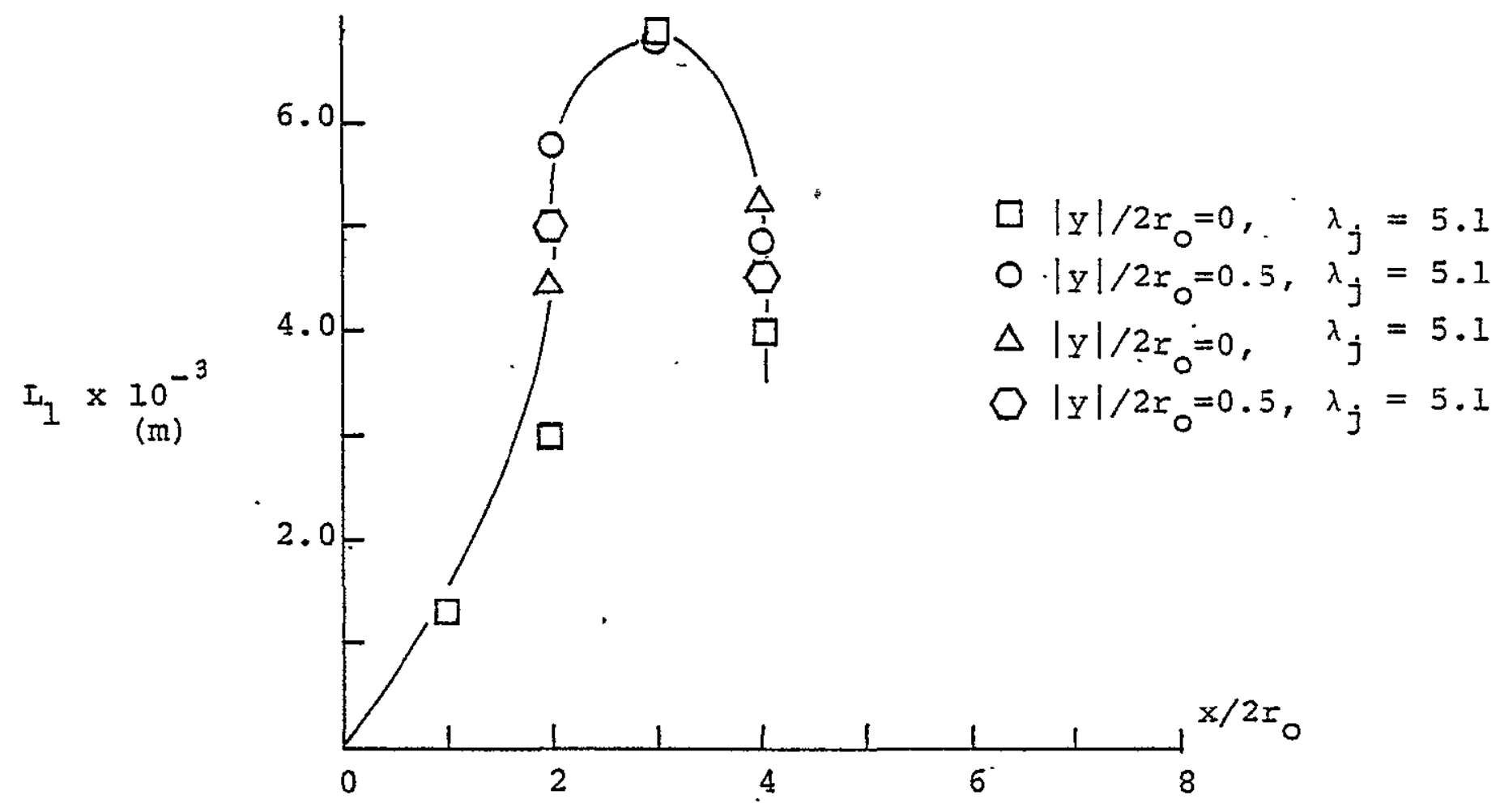

Figure 32 Development of Integral Scale for Jet/Flap, $\mathrm{z} / 2 \mathrm{r}_{\mathrm{o}}=0.5$

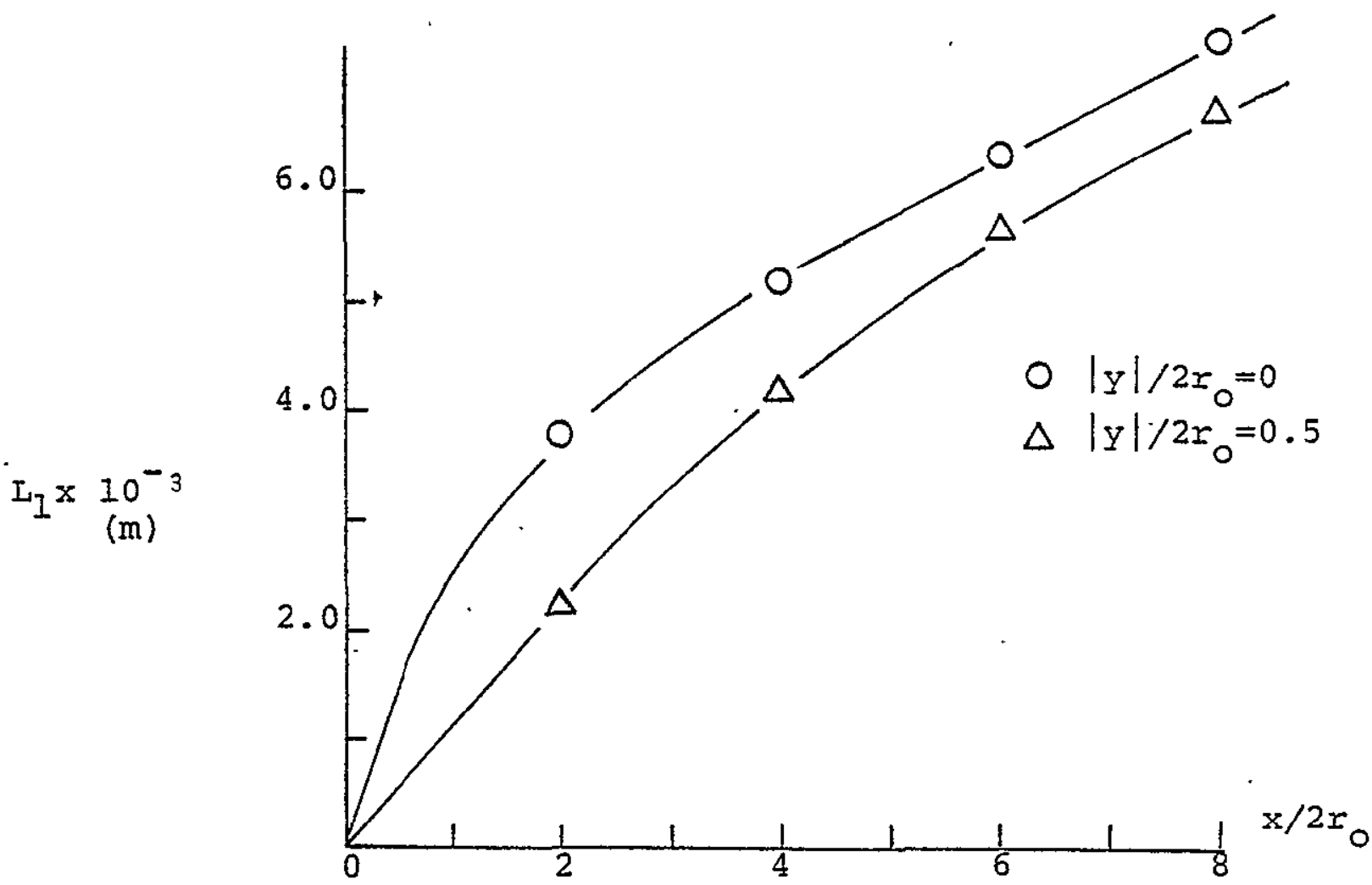

Figure 33(a) Development of Integral Scale for Jet/Plate,

$$
\mathrm{z} / 2 x_{0}=0.5 \quad\left(\lambda_{j}=10.88\right)
$$




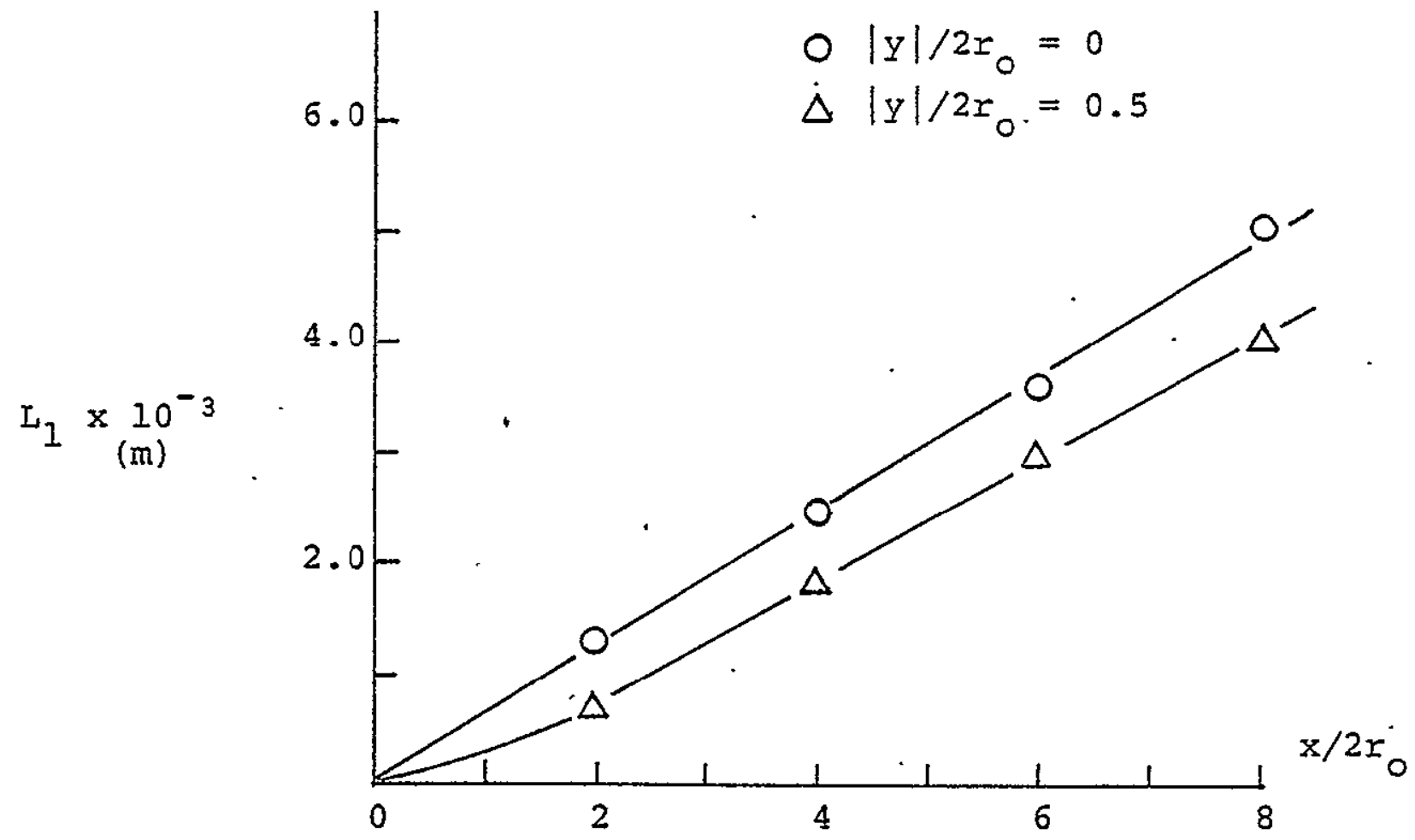

Figure $33(\mathrm{~b})$ Development of Integral scale for Jet/ Plate, $z / 2 r_{0}=0.5 \quad\left(\lambda_{j}=5.1\right)$ 


$$
\left(\frac{d u^{\prime}}{d x}\right)^{2}=\frac{u^{12}}{\lambda_{T^{2}}{ }^{-1}}
$$

For isotropic turbulence, the Taylor microscale can be rewritten:

$$
\frac{I}{\lambda_{T}{ }^{2}}=\frac{I / 5 \int_{0}^{\infty} k^{2} E(k) d k}{\int_{0}^{\infty} E(k) d k}
$$

where $k$ is a wavenumber, and $E^{\prime}(k)$ is the three dimensional energy spectrum function. The integral in the numerator is an indicator of the overall scale of dissipation while the integral in the denominator is an indicator of the overall scale of energy. Thus $\lambda_{\mathrm{T}}$ may be thought of as the ratio of the aissipation of energy to the total amount of energy in flow.

The Taylor microscale can be determined experimentalIy by fitting a parabola to the autocorrelation coefficient curve near the origin. The intersection of the parabola with the delay time axis, along with the use of Taylor's hypothesis yield the value of the Taylor microscale.

Table 1 shows the value of the Taylor microscale for the three flow configurations with $\lambda_{j}$ equal to 5.1 and also equal to 10.88 . For each flow field, the Taylor microscale is determined both at the centerline and at 


\begin{tabular}{|c|c|c|c|c|c|}
\hline & & $\lambda_{j}=5.1$ & & & $=10.88$ \\
\hline & $x / 2 r_{0}$ & $|y|, 2 x_{0}=0$ & $|y| / 2 r_{0}=.5$ & $|y| / 2 r_{0}=0$ & $|\underline{y}| / 2 x_{0}=.5$ \\
\hline jet & $\begin{array}{l}0 \\
2 \\
4 \\
6 \\
8\end{array}$ & $\begin{array}{l}3.52 \times 10^{-3} \\
1.73 \times 10^{-3} \\
1.82 \times 10^{-3} \\
1.88 \times 10^{-3} \\
1.87 \times 10^{-3}\end{array}$ & $\begin{array}{l}6 . \overline{0 \times 10^{-4}} \\
8.6 \times 10^{-4} \\
9.35 \times 10^{-4} \\
12.1 \times 10^{-4}\end{array}$ & $\begin{array}{l}2 .-26 \times 10^{-} \\
2.22 \times 10^{-} \\
-\overline{7} \\
2.75 \times 10^{-}\end{array}$ & $\begin{array}{c}1.92 \times 10^{-4} \\
4.66 \times 10^{-4} \\
=- \\
4.83 \times 10^{-4}\end{array}$ \\
\hline & & $\lambda_{j}=5.1$ & & & $=10.88$ \\
\hline & $x / 2 r_{0}$ & $|y| / 2 \Sigma_{0}=0$ & $|y| / 2 r_{0}=.5$ & $|y| / 2 x=0$ & $|y| / 2 \Sigma_{0}=.5$ \\
\hline Jet/Plate & $\begin{array}{l}2 \\
3 \\
4 \\
6 \\
8\end{array}$ & $\begin{array}{l}3.41 \times 10^{-4} \\
6.8 \times 10^{-4} \\
7.3 \times 10^{-4} \\
7.66 \times 10^{-4} \\
1.64 \times 10^{-3}\end{array}$ & $\begin{array}{l}6.74 \times 10^{-4} \\
1.32 \times 10^{-3} \\
3.13 \times 10^{-3} \\
3.55 \times 10^{-3} \\
4.01 \times 10^{-3}\end{array}$ & $\begin{array}{l}7.56 \times 10^{-4} \\
9.15 \times 10^{-4} \\
9.52 \times 10^{-4}\end{array}$ & $\begin{array}{l}5.69 \times 10^{-4} \\
6 . \overline{4 \times 10^{-4}} \\
6 . \overline{8} \times 10^{-4}\end{array}$ \\
\hline & & ${ }^{\lambda_{j}}=5.1$ & . & & $=10.88$ \\
\hline & $x / 2 x_{0}$ & $|y| / 2 r_{0}=0$ & $|y| / 2 r_{0}=.5$ & $|y| / 2 r_{0}=0$ & $|y| / 2 r_{0}=.5$ \\
\hline Jet/Flap & $\begin{array}{l}1 \\
2 \\
3 \\
4\end{array}$ & $\begin{array}{l}3.96 \times 10^{-4} \\
1.23 \times 10^{-3} \\
6.31 \times 10^{-4} \\
5.1 \times 10^{-4}\end{array}$ & $\begin{array}{l}8.4 \times 10^{-4} \\
6.86 \times 10^{-4} \\
4.4 \times 10^{-4}\end{array}$ & $\begin{array}{l}1.77 \times 10^{-3} \\
6.77 \times 10^{-4}\end{array}$ & $\begin{array}{l}9.55 \times 10^{-4} \\
5.02 \times 10^{-4}\end{array}$ \\
\hline
\end{tabular}

TABLE 1. Comparison of Taylor Microscales 
the lateral position corresponding to the Iip of the nozzle.

Consider initially the microscales determined for the unconfined turbulent jet. The values shown for the centerline position are fairly constant though there does seem to be a slight tendency to increase. Downstream from the lip of the nozzle, that tendency is seeringly stronger. It is interesting to note that the values of $\lambda_{T}$ at the centerline when $\lambda_{j}$ is equal to 5.1 are consistently lower than correpsonding values when $\lambda_{j}$ equals 10.88. The opposite is the case, however, downstream from the lip of the nozzle.

Next, the case of the flow of the jet over the plate will be discussed. Here again, a slight increase in the value of the Taylor microscale is noted as the flow progresses downstream. The value of $\lambda_{j}$ does seem to have a strong influence on the value of $\lambda_{\text {T }}$ downstream from the lip of the nozzle. With $\lambda_{j}$ equal to 10.88 the values of $\lambda_{T}$ out in the shearing layer are almost an order of magnitude different than for the corresponding position for the smaller value of $\lambda_{j}$.

Finally, consider the flow of the turbulent jet over the flap. Here at the centerline and the shearing layer location, the Taylor microscale first increases and then actually decreases in value. This is the case for both 
ratios of inner jet velocity to outer tunnel flow speed. Since the Taylor microscale may be thought of as the ratio of the dissipation of energy to the total amount in the flow, consider the implication of $\lambda_{T}$ decreasing. A decrease in the magnitude of the Taylor microscale represents a decrease in relative magnitudes of the total energy in the flow to the dissipation in the flow. Considering the total energy aspect first, since the turbulence obtains its energy from the mean velocity, a decrease in the mean velocity will eventually show up as a decrease in the total energy of the flow. With respect to the relative increase in the dissipation of energy, recall that the dissipation is dominant at high frequencies. The flap which serves to amplify the turbulence in the flow field quite rapidly enhances the energy cascading process and, thus, could be.responsible for the relative increase in the magnitude of the dissipation. Evidence thus points to the fact that the flap has a very strong influcence on the turbulent velocity field.

Section 4.5 Power Spectral Densities

To determine the spectral density curves, a computer program is used to take the Fourier Transform of the autocorrelation functions. The power spectra are nor- 
malized so that the area underneath each curve is 1.

Initially, data is presented for the three different flow configurations with $\lambda_{j}$ set equal to 5.1 . In Figure 34, the spectral density curves both at the centerline $\left(|y| / 2 r_{0}=0\right)$ and corresponding to the lateral position of the Iip of nozzle $\left(|y| / 2 r_{0}=0.5\right)$ are shown for a downstream displacement of two diameters and with $z / 2 r_{0}=0.5$. The power spectrum in the potential core of a turbulent jet is characterized by a large "bump" or peak. (62) (38) At the centerline the peak of the unconfined jet is clearIy evident. In fact, as would be expected from observing. the autocorrelation curve for this location the power spectra of both the flows over the plate and flap are also characterized by a large peak. For the flow over the plate, the peak has approximately the same magnitude as the unconfined flow but with a slight shift in frequency. With respect to the flow over the flap, the peak has been markedly diminished and, again, shifted to an even higher frequency. In the shearing layer, the distinctions among the three configurations are no longer evident. In this region, the peak is virtually entirely masked out by the relatively high turbulence. levels.

Power spectra for the downstream location of $x / 2 x_{0}=$ 4 and $z / 2 r_{0}=0.5$ are reported for the three fllow configurations at the centerline and nozzle lip lateral 


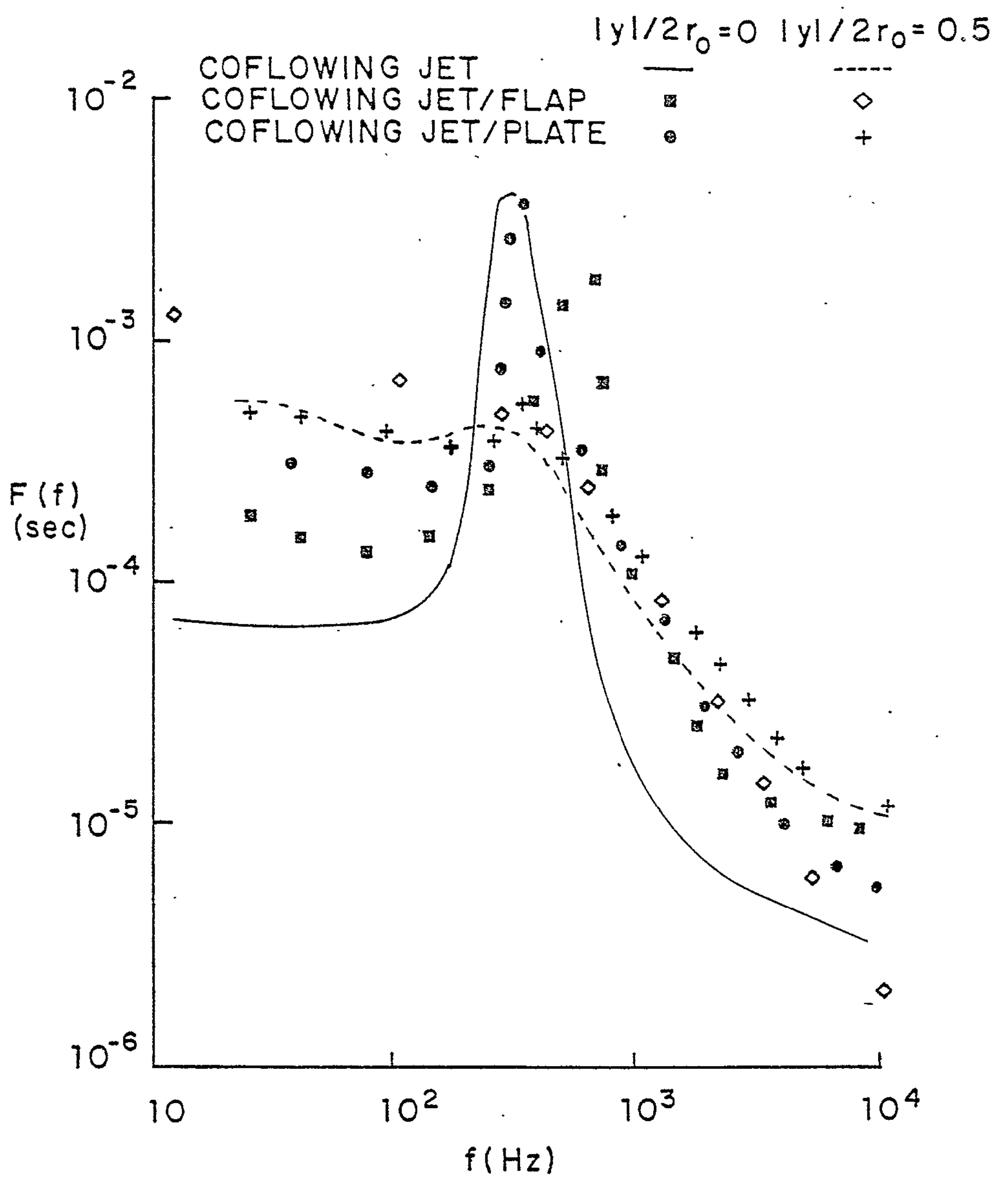

Figure 34 Power Spectral Density Comparison, $x / 2 r_{0}=2$, $z / 2 r_{0}=0.5 \quad\left(\lambda_{j}=5.1\right)$ 
positions in Figure 35. For the unconfined jet, the potential core region of the jet still exists and is characterized by a large peak, slightly attenuated and shifted to a higher frequency than was the case at $x / 2 r_{0}=$ 2. For the case of the flow over the plate, the peak is also present though with a smaller magnitude than for the unconfined jet and at a slightly higher frequency. Turning to the flow over the flap, the strong effect the curved wall surface has on the turbulent velocity field is clearly demonstrated. Notice that the characteristic peak is totally absent.

Finally just as was seen two diameters downstream of the lip, the power spectra in the shearing region are quite similar for all three flow field configuration. With the purposes of viewing the change in the power spectra for each configuration from a slightly different perspective and also examining the effects.of-varying the ratio of the inner jet velocity to the outer tunnel speed, power spectral densities for the three flow field are presented with $\lambda_{j}$ set equal to 10.88. The format chosen for display of the data is identical to that used for the autocorrelation coefficient curves with $\lambda_{j}$ equal to 10.88. For each flow configuration, one set of power spectra corresponding to the centerline location $\left(|y| / 2 r_{0}=\right.$ $0)$ is shown as well as a second set for the lateral 


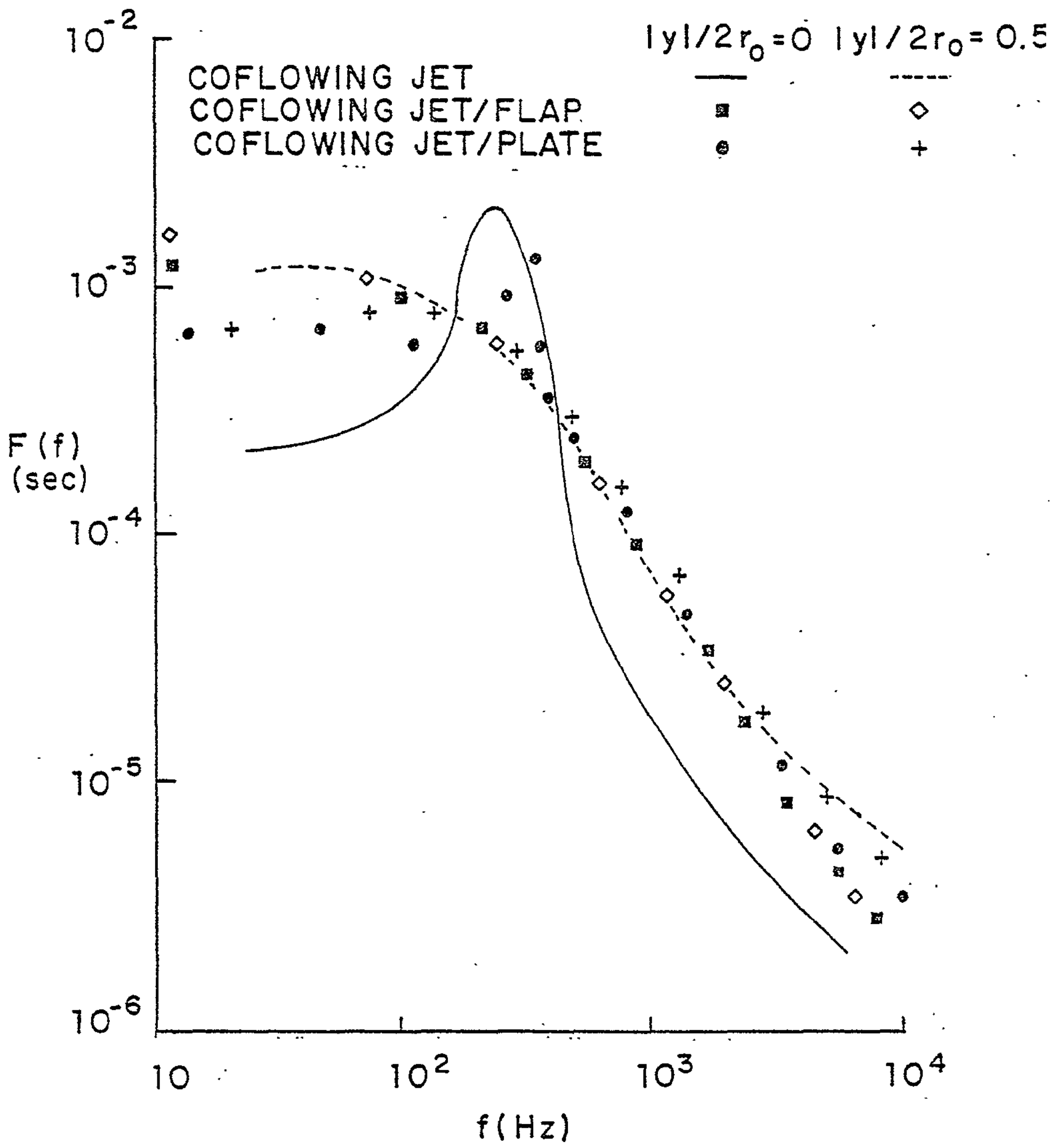

$\begin{aligned} \text { Figure } 35 & \begin{array}{l}\text { Power Spectral Density Comparison, } x / 2 r_{0}=4, \\ \mathrm{z} / 2 r_{0}=0.5\end{array}\end{aligned}$ 
location directly downstream from the nozzle lip $\left(|y| / 2 r_{0}=\right.$ $0.5)$.

Focussing initially on the development of the unconfined coflowing jet, the characteristic large peak in the spectrum is clearly evident at $x / 2 r_{0}=2$ (Figure 36). This peak is slightly attenuated and broadened four diameters downstream of the exit plane. At $x / 2 x_{0}=8$, the power spectrum has lost its peak and seems to be approaching a fully developed turbulent nature. Turning back to the data presented for the flow with $\lambda_{j}$ equal to 5.1 , the peak is at the same frequency and the relative decay of the "bump" in the spectra behaves similarly. In Figure 37, the change in the power spectra in the mixing region. is shown. There seems to be a shift of energy from the high frequency components to the lower frequency components of the spectrum. Here again this is the same behavior found when $\lambda_{j}=5.1$. The development of the power spectra for the flow over the plate is shown in Figures 38 and 39 . At the centerline location, the power spectrum had a peak similar to that found for the unconfined jet. However, as was noted for the case when $\lambda_{j}=5.1$, the peak is both attenuated and shifted to a higher frequencies. Notice that again, the peak in the power spectrum broadens as the flow progresses from two to four diameters down- 


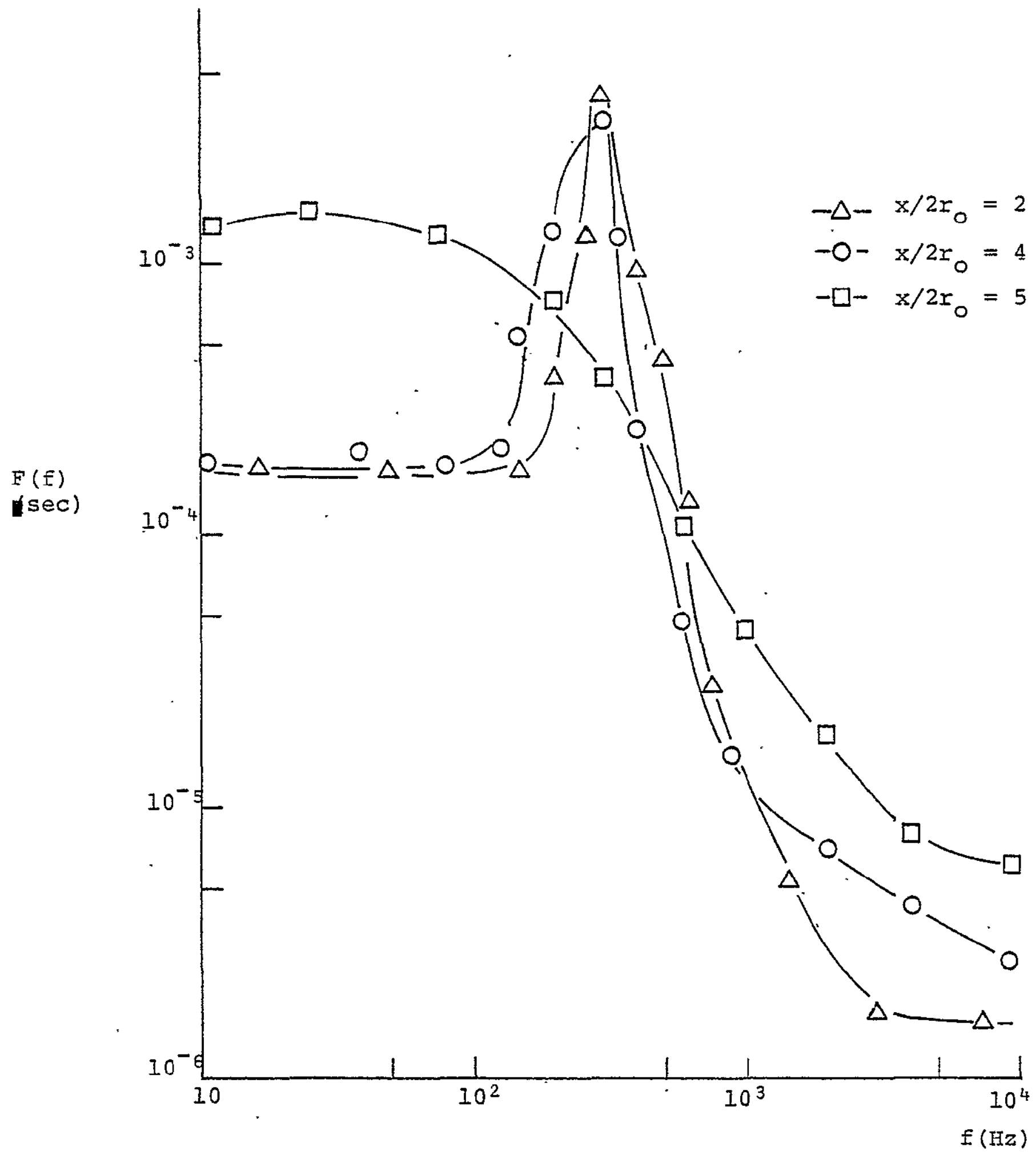

Figure 36 Downstream Development of Power Spectral Density for Jet, $|y| \neq 2 r_{0}=0, z / 2 x_{0}=0.5$

$$
\left(\lambda_{j}=10.88\right)
$$




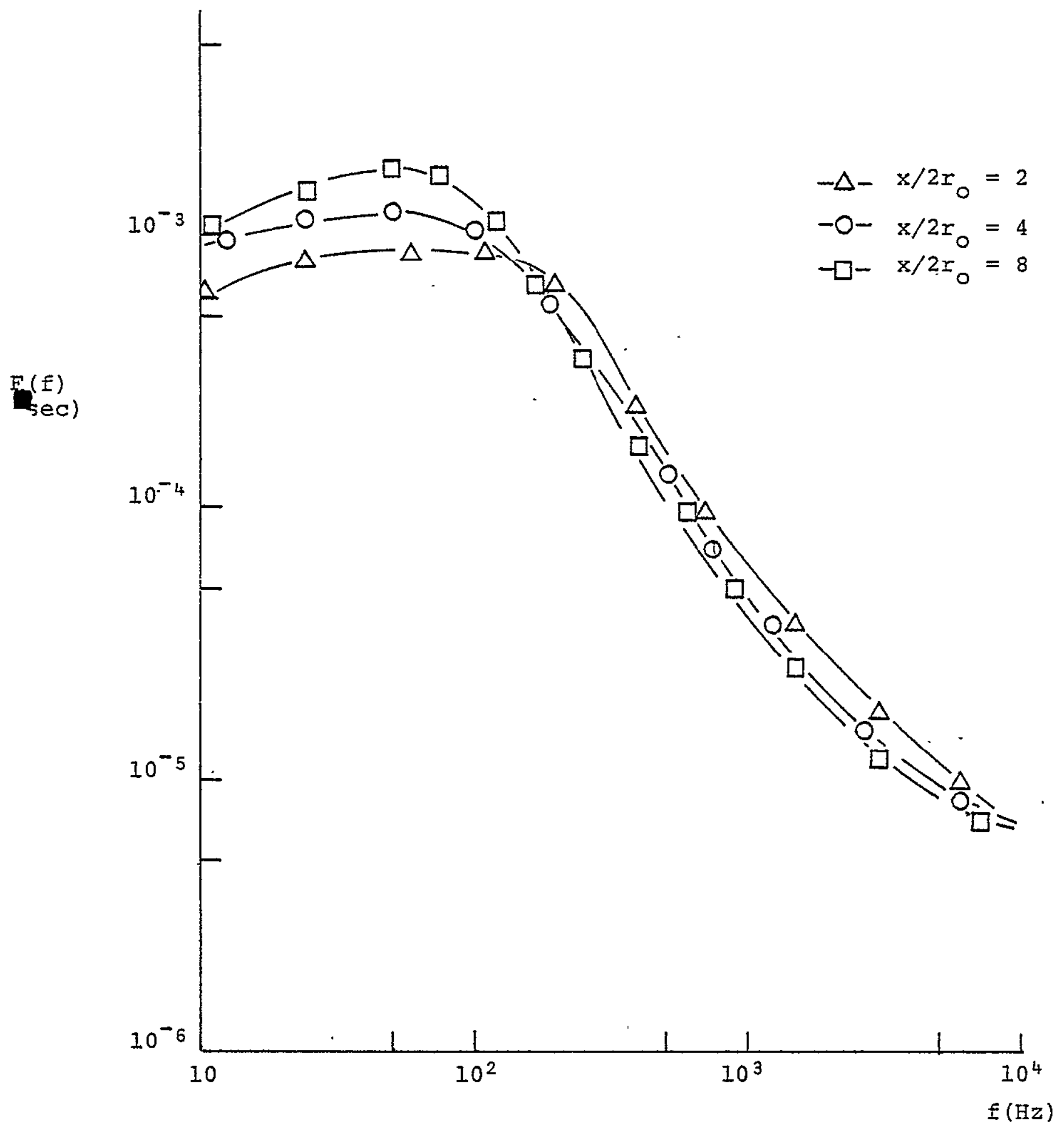

Figure 37 Downstream Development of Power Spectral Density for Jet, $|y| / 2 r_{0}=0.5, z / 2 r_{0}=0.5$

$$
\left(\lambda_{j}=10.88\right)
$$




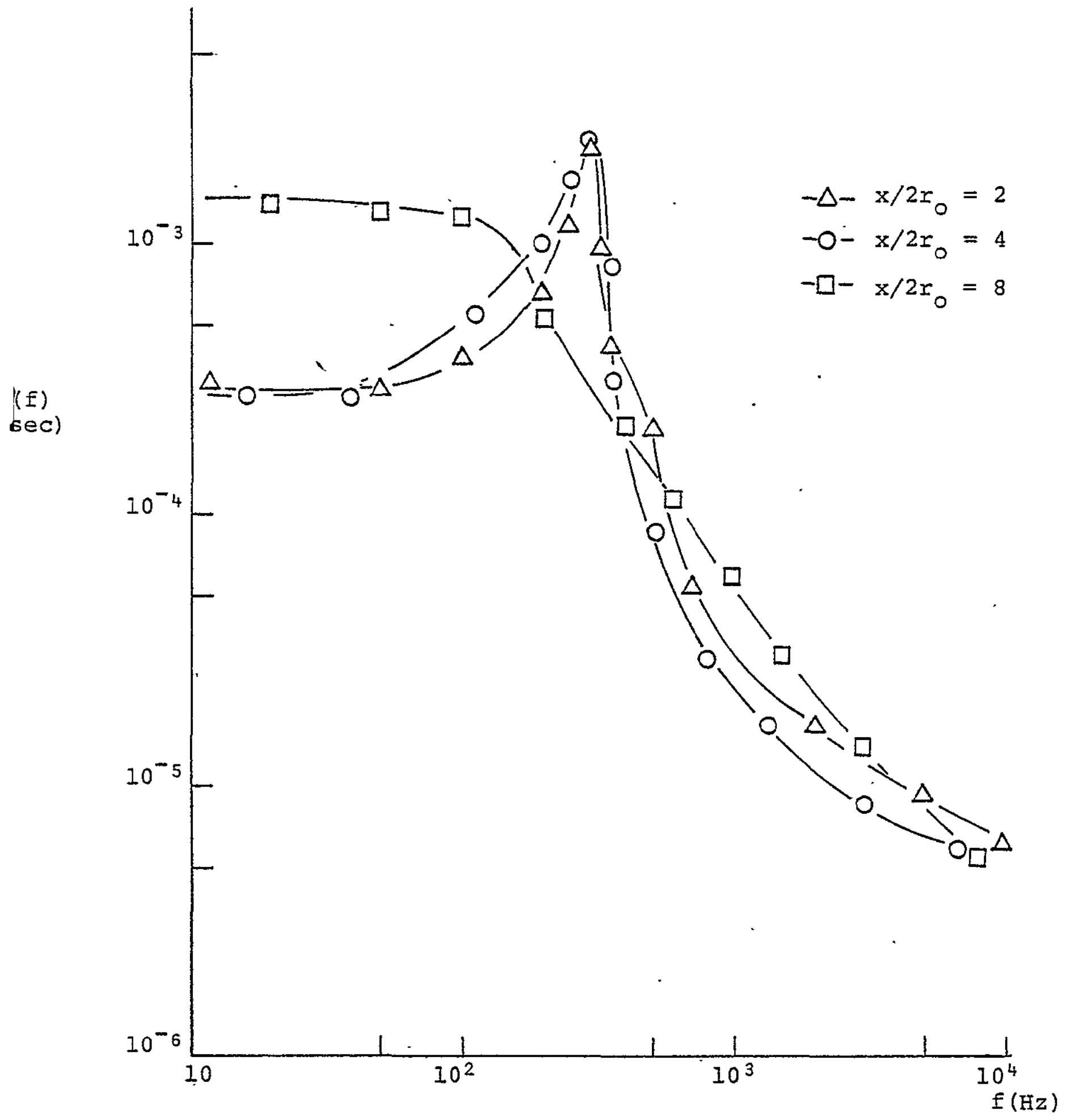

Figure 38 Downstream Development of Power Spectral Density for Jet/Plate, $|y| / 2 r_{0}=0, z / 2 x_{0}=0.5$

$$
\left(\lambda_{j}=10.88\right)
$$




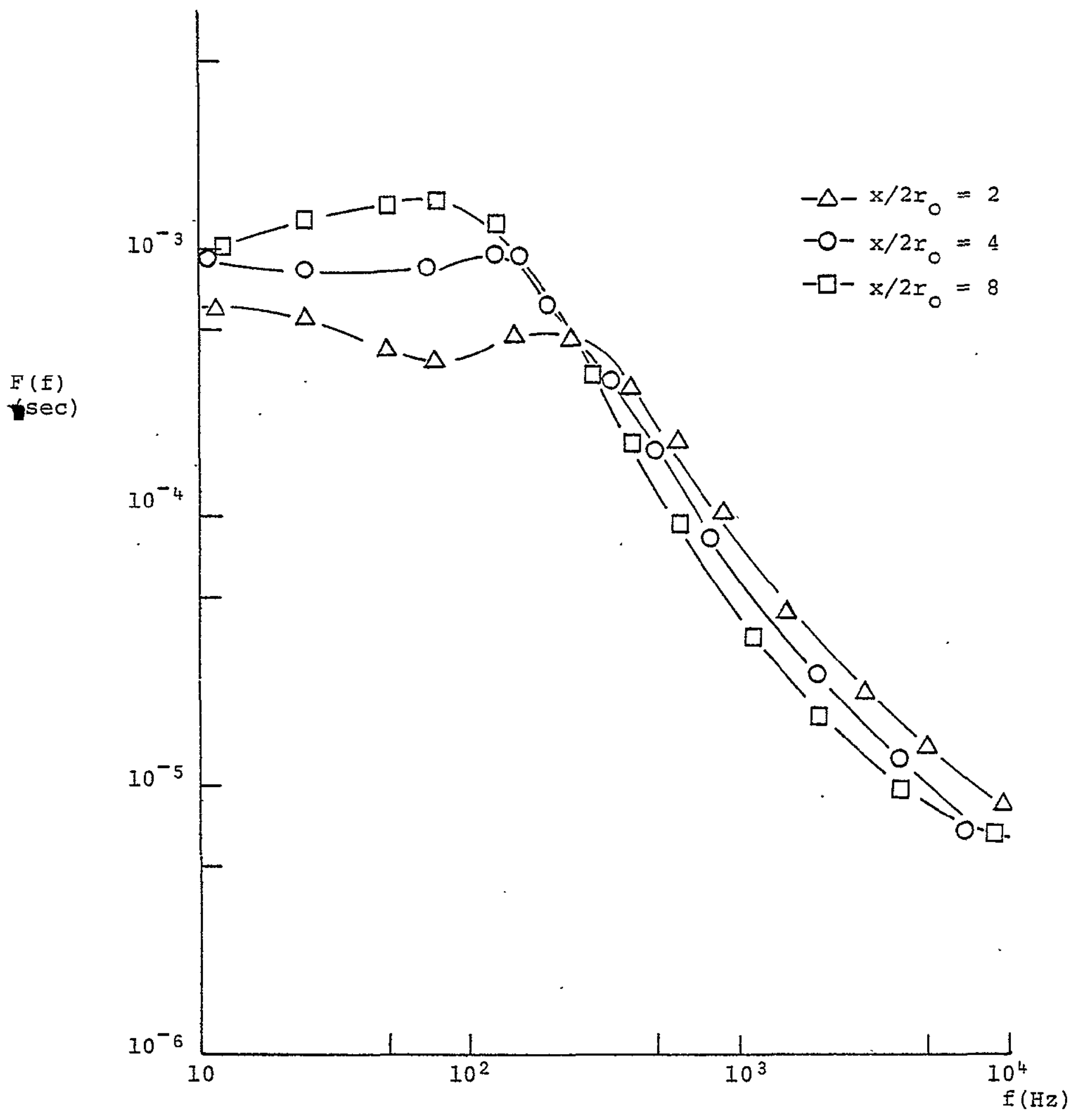

Figure 39 Downstream Development of Power Spectral Density for Jet/Plate, $|y| / 2 r_{0}=0.5, z / 2 r_{0}=0.5$

$$
\left(\lambda_{j}=10.88\right)
$$


stream from the exit plane, and at $x / 2 x_{0}=8$, the "bump" is absent. The dominant frequencies in the spectrum when $\lambda_{j}=5.1$ and when $\lambda_{j}=10.88$ are the same. Out in the mixing region (Figure 39) there also is a shift of power from higher frequency components to the lower frequency components of the spectrum. This behavior is quite similar to that exhibited by the unconfined jet.

Finally, power spectra for the turbulent jet flowing over the flap are presented in Figure 40 and 41 . As might be expected, the large peak in the power spectrum is absent at $x / 2 r_{0}=4$ at the centerline of the flow field. However, there is one difference for the case where $\lambda_{j}$ is. equal to 10.88 and this occurs at $x / 2 r_{0}=2$. With $\lambda_{j}$ equal to 5.1 , the peak in the spectrum is slightIy attenuated and the frequency is shifted to higher frequencies as compared to the case of the unconfined jet. For $\lambda_{j}$ equal to 10.88 , neither of these changes occur. In the mixing region of the flow field, the shift of energy from high to low frequencies is apparent.

\section{Section 4.6 Intermittency}

The percent time of the occurrence of turbulence at any point within the flow field is called the intermittency factor (I). It has been demonstrated by Van Atta, ${ }^{(63)}$ Shaughnessy ${ }^{(58)}$ and Catalano, et. al. (34) that 


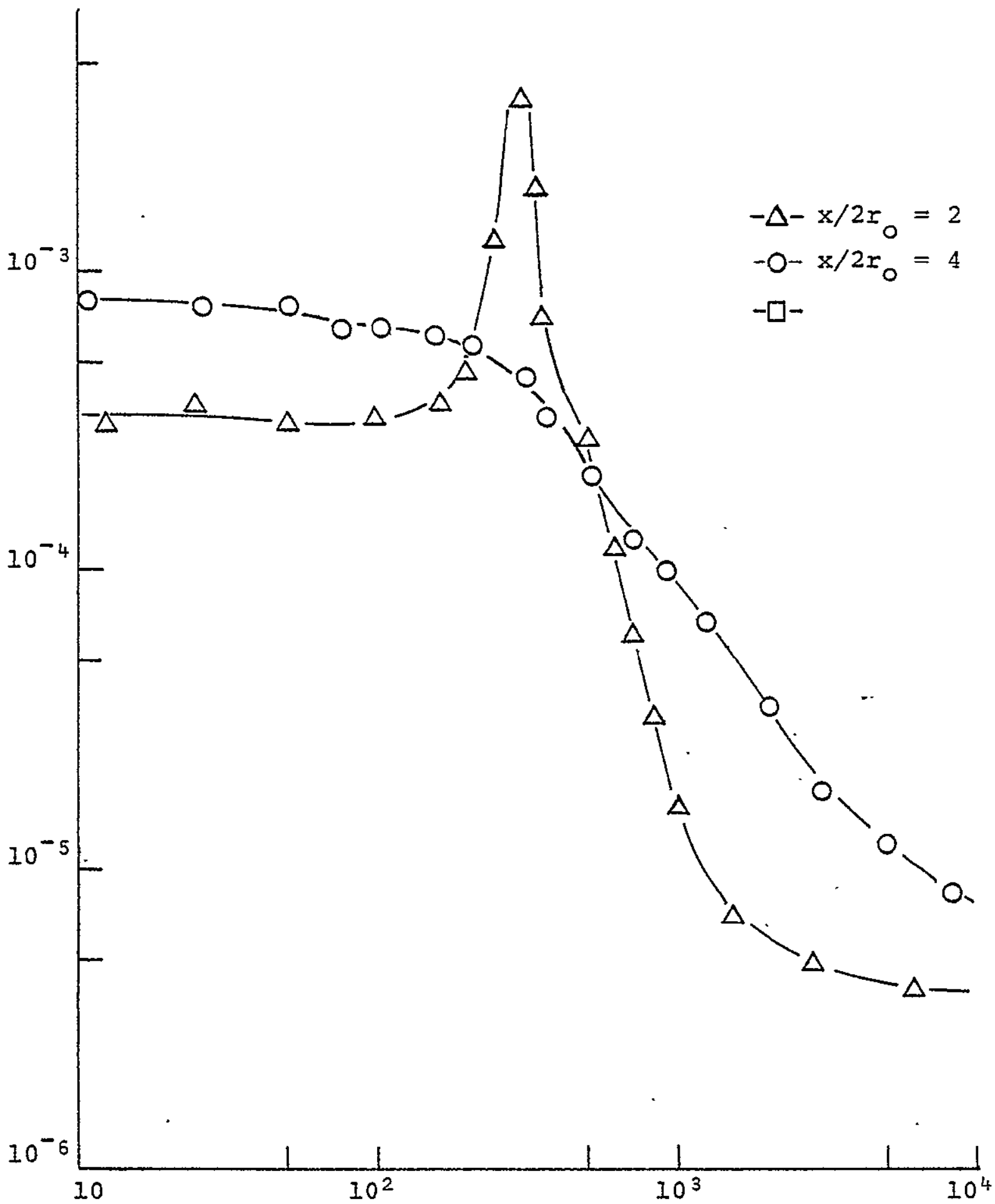

Figure 40 Downstream Development of Power Spectral Density for Jet/Flap, $|y| / 2 r_{0}=0, z / 2 r_{0}=0.5$

$$
\left(\lambda_{j}=10.88\right)
$$




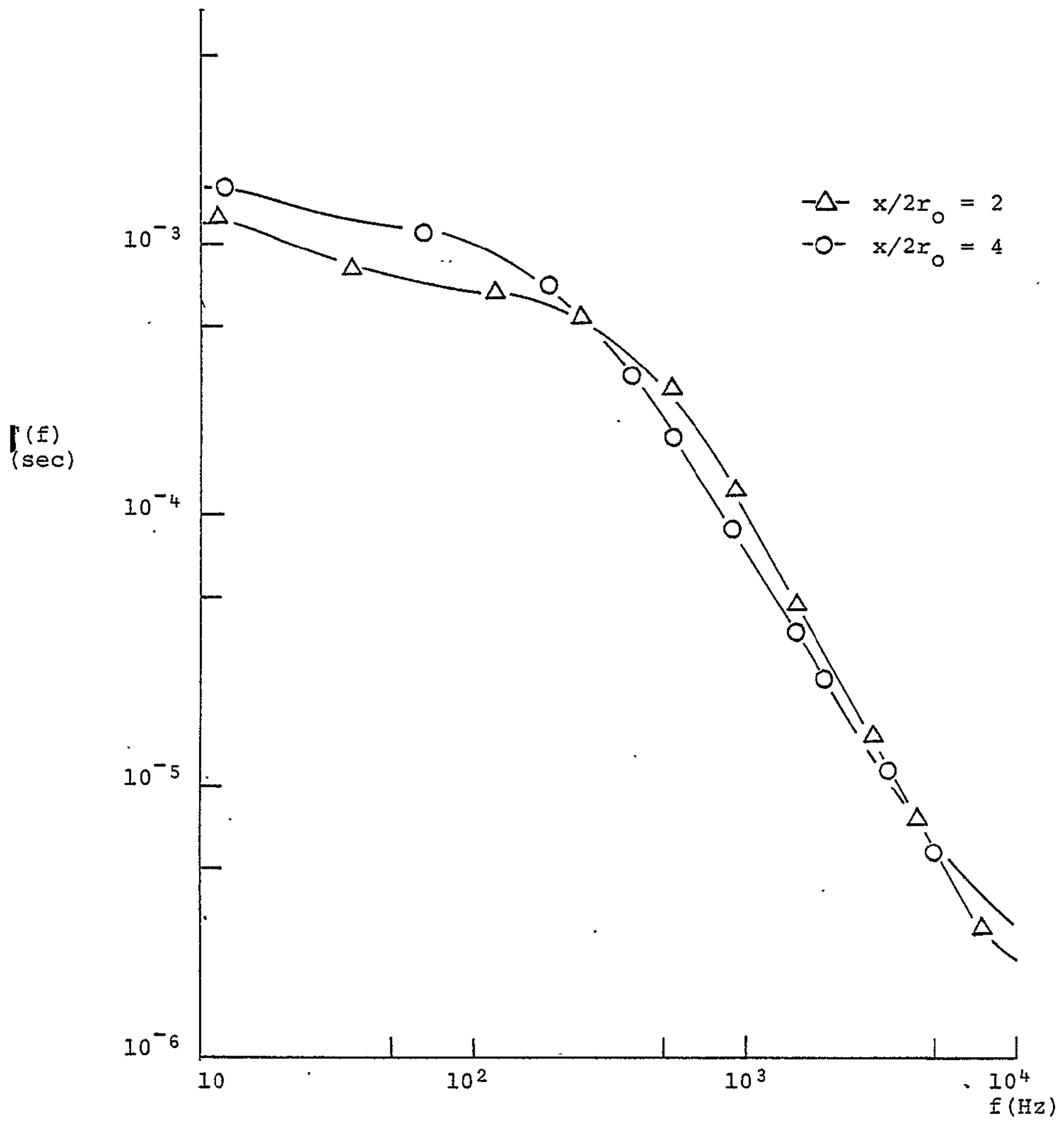

Figure 41 Downstream Development of Power Spectral Density for Jet/FIap, $|y| / 2 r_{0}=0.5, z / 2 r_{0}=0.5$

$$
\left(\lambda_{j}=10.88\right)
$$


the intermittency of a given flow field can be determined by monitoring the diffusion of any seeding particles as long as those marking particles are passive and provided the Schmidt number is relatively high. The schmidt number, which is approximately $10^{4}$ for this experiment, is defined as the ratio between the kinematic viscosity of the fluid and the diffusion coefficient of the seeding particles (in this case, DOP). Thus the schmidt number relates viscous diffusion to mass diffusion. Profiles for intermittency are presented for the region outside the lip of the nozzle. The radial and axial distributions of the intermittency factor at various vertical locations for the unconfined jet flow and the flows over the flap and plate are given in Figure 42. All the data shown is for the case when $\lambda_{j}$ is equal to 5.1 .

For the vertical location $z / 2 r_{0}=0.5$, the flow field with the flap in place is significantly wider than either the unconfined jet or the plate case. It is interesting to note, however, that after the very large initial increase in the width, the flow field actually decreases in width as the turbulent jet progresses downstream. Thus as the flow starts to be turned by the flap, the width of the flow field at $z / 2 r_{0}=0.5$ decreases. 


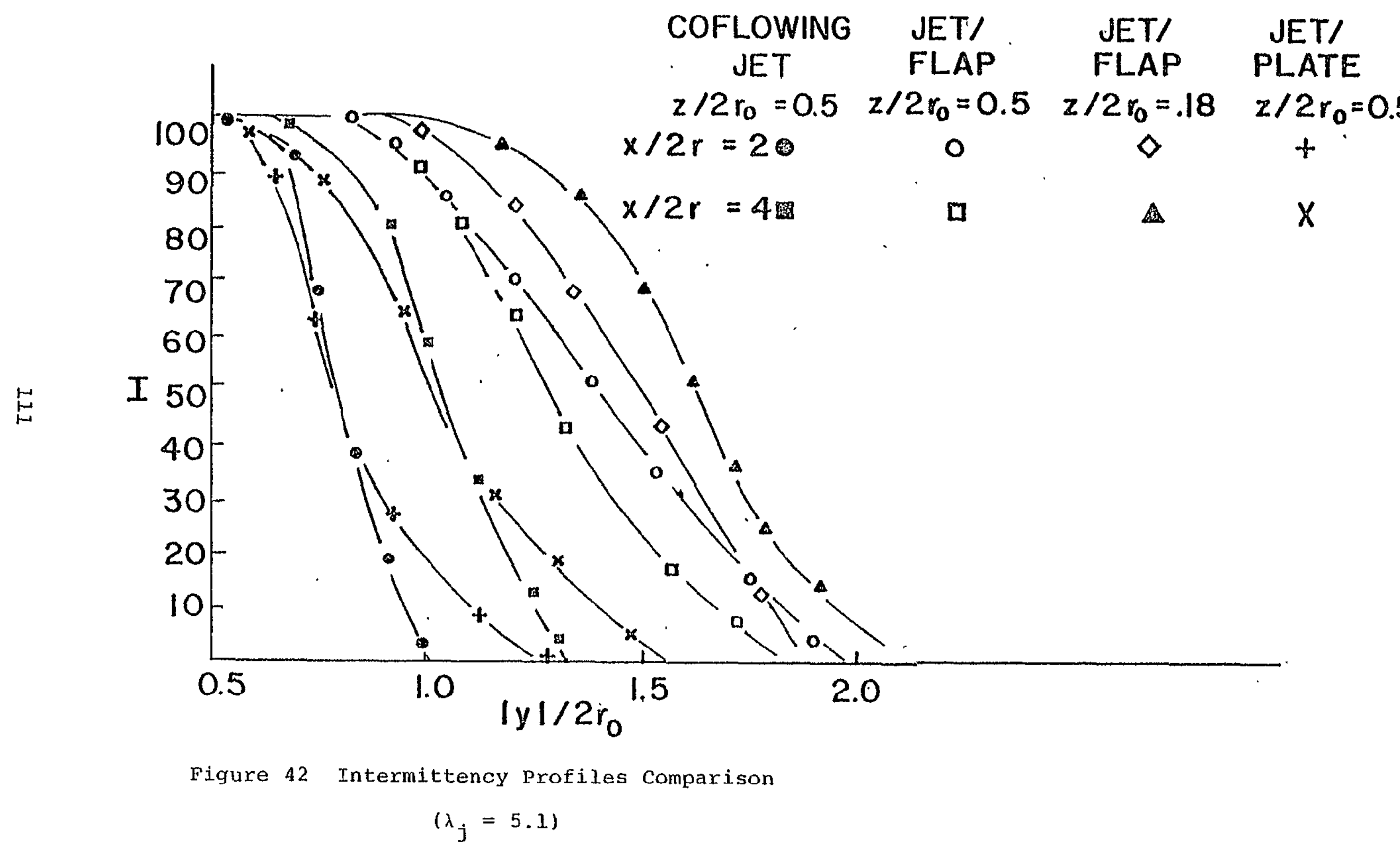


For the vertical position $z / 2 r_{0}=0.18$, the turbulent jet flowing over the flap is greater in width at each downstream location than it was at $x / 2 x_{0}=0.5$ and it also increases in size as the jet progresses downstream. The flap seemingly acts to draw the jet down towards the surface and increase the flow field's width. It also serves to transform what was an axisymmetric jet into a flow characterized by a rather elongated oval type cross section.

In the discussion of the mixing widths for the flow fields, it was mentioned that they were about the same for both the unconfined jet and the flow over the plate. Here that discussion is reinforced by comparing the intermittency profiles at $z / 2 r_{0}=0.5$. The profiles are quite similar in shape.

\section{Concentration Auto and Cross Correlations}

The concentration auto and cross correlations are defined in a similar manner as those determined for the velocity field. However, it is the fluctuations of the passive admixture (DOP) which is being monitored rather than the turbulent velocity fluctuations. The autocorrelation function of the concentration field is defined as follows:

$$
R_{C}=\frac{\overline{C C^{*}}}{\left(\bar{c}^{2}\right)^{\frac{1 / 2}{2}\left(\overline{c^{*}}\right)^{\frac{1}{2}}}}
$$


where

$$
c=c(x, y, z, t) \text { and } c^{*}=c(x, y, z, t+\Delta t)
$$

Consider the cross-correlation function of the concentration field defined in the restricted manner such that only lateral or $y$ displacements are allowed between the two measuring points. Then the cross correlation takes the following form:

$$
\operatorname{Rc}_{12} c_{2}=\frac{\overline{c_{1}(x, y, z, t) c_{2}(x, y+\Delta y, z, t+t)}}{\left(\bar{c}_{1}^{2}\right)^{\frac{1}{2}}\left(\bar{c}_{2}^{2}\right)^{\frac{1}{2}}}
$$

where $c_{1}$ and $c_{2}$ are the concentration fluctuations at two different points in the flow field.

Next, the further restriction is made that the concentration fluctuations will be monitored only at the lateral positions corresponding to the lip of nozzle $\left(y / 2 r_{0}= \pm 0.5\right)$ at the vertical location $z / 2 r_{0}=0.5$. Autocorrelations and cross correlations are measured in the three flow configurations at different downstream locations. Consider initially the case of the unconfined coflowing jet. Data is presented for $x / 2 r_{0}=4$ and $x / 2 x_{0}=8$ in Figures 43 and 44 , respectively. At $x / 2 r_{0}=4$, the autocorrelation of the concentration fluctuation is quite similar to the velocity spectia discussed earlier in this investigation. The curve demonstrates a damped sinusoidal behavior. The cross correlation curve is 


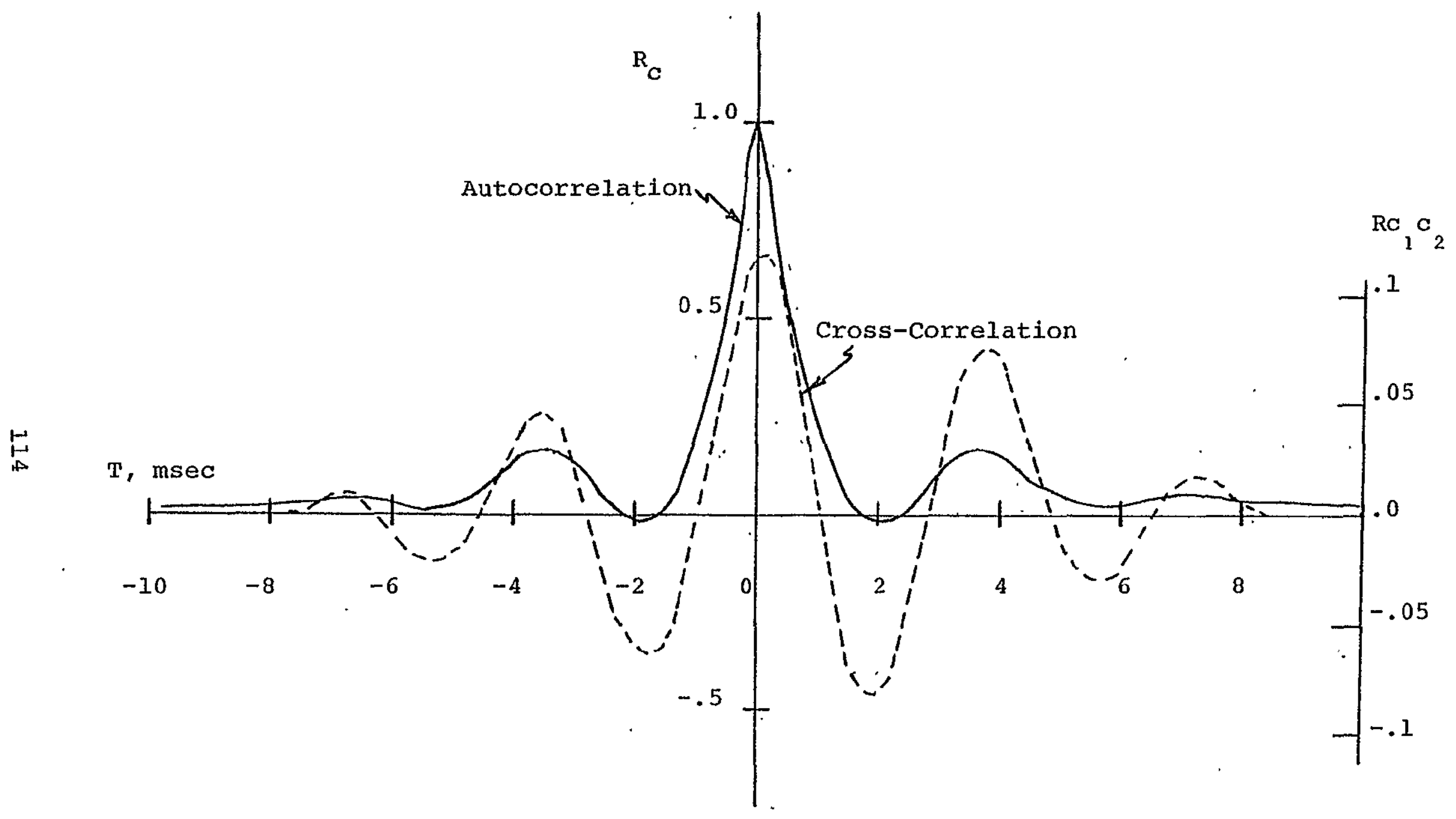

Figure 43 Auto and Cross Correlations for Jet Concentration Field, $x / 2 r_{0}=4$, $y / 2 x_{0}= \pm 0.5, z / 2 x_{0}=0.5 \quad\left(\lambda_{j}=5: 1\right)$ 


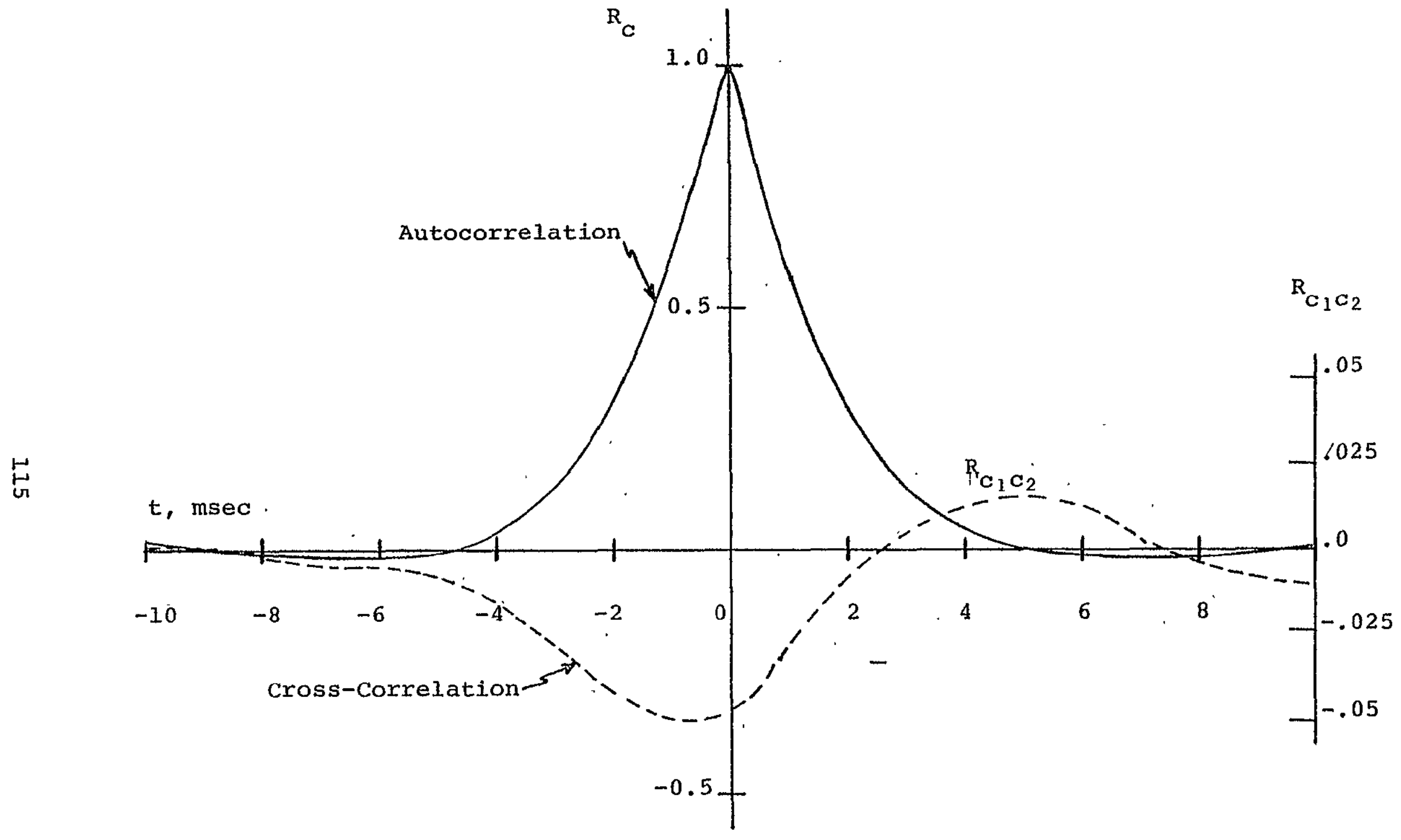

Figure 44 Auto and Cross Correlations for Jet Concentration Field, $\mathrm{x} / 2 \mathrm{r}_{\mathrm{o}}=8, \mathrm{y} / 2 \mathrm{r}_{\mathrm{o}}= \pm 0.5, \mathrm{z} / 2 \mathrm{r}_{0}=0.5 \quad\left(\lambda_{j}=5.1\right)$ 
also of a similar shape. The maximum absolute value of the coefficient $\left(\mathrm{RC}_{1} \mathrm{C}_{2}=.12\right)$ occurs at approximately zero delay time though there is evidence of a slight phase shift between the two concentration signals. At $x / 2 r_{0}=8$ the autocorrelation curve is of a more typically fully turbulent nature. The maximum absolute value of the cross correlation coeficient has significantly decreased $\left(\mathrm{RC}_{1} \mathrm{C}_{2}=.05\right)$ though there still is some relation between the fluctuating concentration field at $y= \pm 0.5$. A large shift in the phase angle between the signals has also occured.

Auto and cross correlations for the flow over the plate are presented in Figures 45 and .46 for the downstream positions, $x / 2 r_{0}=4$ and 8 . The autocorrelation coefficient curve at $x / 2 r_{0}=4$ is quite similar to that found for the case of the unconfined jet at $x / 2 x_{0}=4$. The maximum absolute value of the cross correlation coefficient is similar to the previous case also $\left(R_{1} c_{2}=.13\right.$ ). -The delay time for the maximum coefficient value has been shifted positively. The concentration signals are considerably more out of phase than is the case for the unconfined jet. At $x / 2 r_{0}=8$, the damped sinusoidal nature of the autocorrelation coefficient curve has disappeared. The maximum absolute value of the coefficient has decreased $\left(\mathrm{RC}_{I_{2}} \mathrm{C}_{2}=.04\right)$. As in the case of the unconfined 


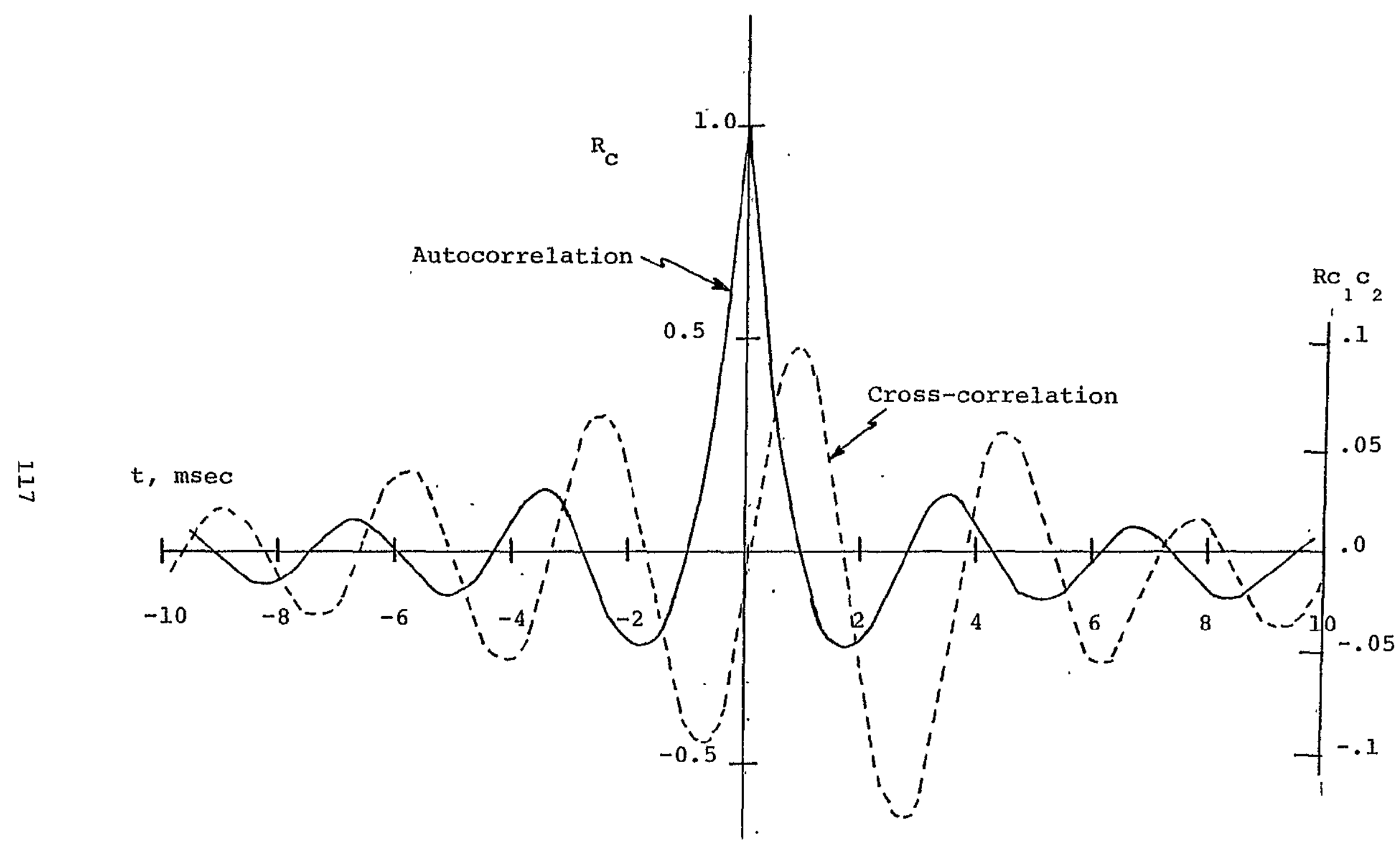

Figure 45 Auto and Cross Correlations for Jet/Plate Concentration Field $\mathrm{x} / 2 \mathrm{r}_{\mathrm{o}}=4, \mathrm{y} / 2 \mathrm{x}_{\mathrm{o}}= \pm 0.5, \mathrm{z} / 2 \mathrm{x}_{\mathrm{o}}=0.5 \quad\left(\lambda_{\mathrm{j}}=5.1\right)$ 


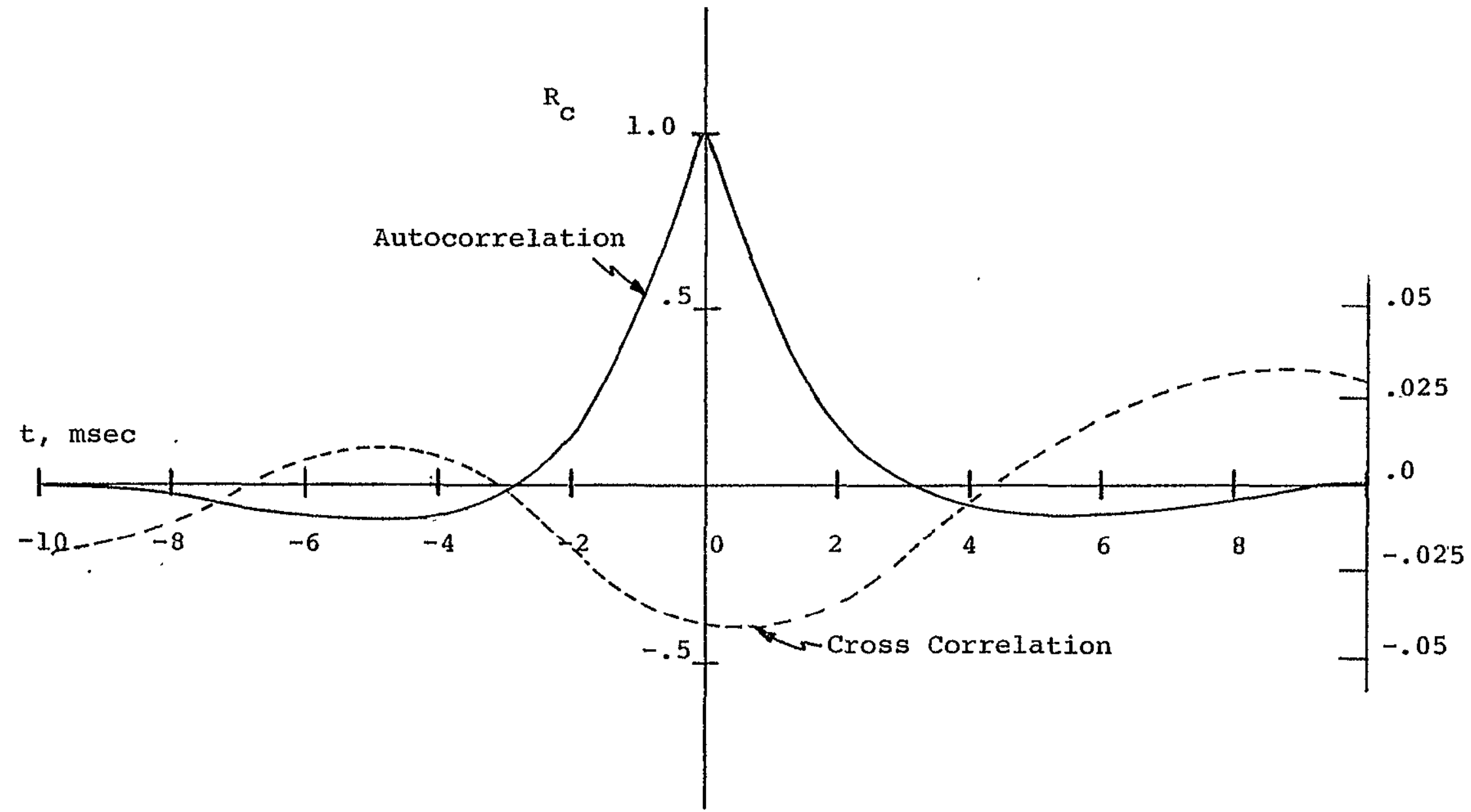

Figure 46 Auto and Cross Correlations for Jet/Plate Concentration Field $\mathrm{x} / 2 \mathrm{r}_{\mathrm{o}}=8, \mathrm{y} / 2 \mathrm{r}_{\mathrm{o}}= \pm 0.5, \mathrm{z} / 2 \mathrm{r}_{\mathrm{o}}=0.5 \quad\left(\lambda_{j}=5.1\right)$ 
jet, though the concentration autocorrelation curves appear to fully turbulent in nature, there still is evidence of a relationship (albeit a weak one) between the fluctuations at each lip of the nozzle.

Finally, consider the flow of the jet over the flap. Auto and cross correlations at $x / 2 r_{0}=4$ are presented in Figure 47. The autocorrelation coefficient is quite similar in shape to the previous two cases for this downstream location. The maximum absolute value of the cross correlation coefficient is also comparable $\left(R C_{1} C_{2}=.12\right)$. The cross correlation coefficient curve, much like the case for the flow over the plate, demonstrates a large phase shift between the concentration fluctuations at $\mathrm{y} / 2 \mathrm{r}_{0}= \pm 0.5$.

Concentration Field Spectra

A spectrum of concentration or contaminant fluctuations is produced when smoke particles are mixed by the turbulent flow field of the jet. Many of the same concepts that are used in describing the kinetic energy spectrum can be applied to the spectra of the mixing smoke particles. The scales of the smoke particles fluctuations range from the scale of the energy-containing eddies to a smallest scale that is dependent on the ratio of the diffusivities (Schmidt number). The spectra are normalized for unit area. 


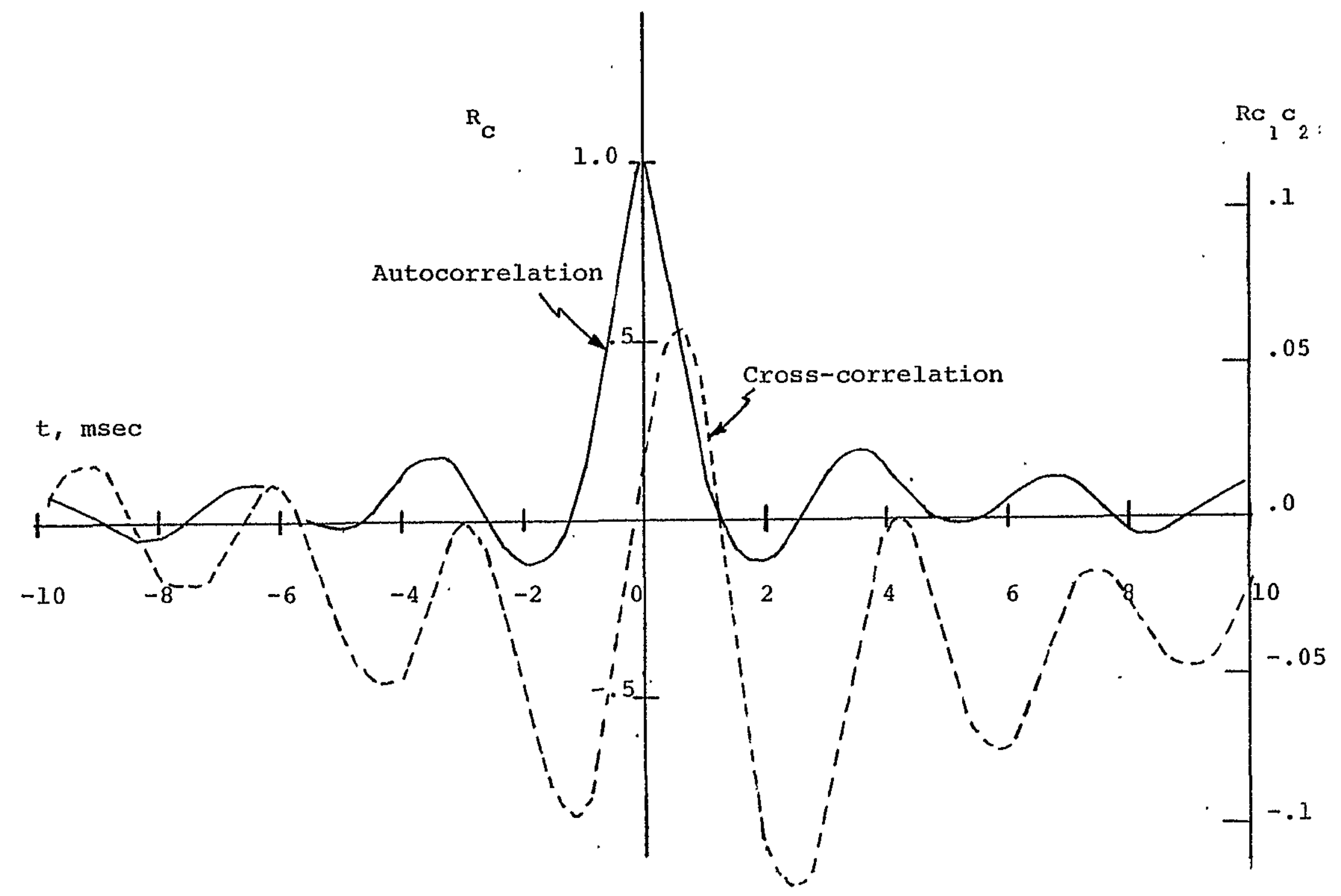

Figure 47 Auto and Cross Correlations for Jet/Flap Concentration Field, $\mathrm{x} / 2 r_{\mathrm{o}}=4, \mathrm{y} / 2 \mathrm{r}_{\mathrm{o}}= \pm 0.5, \mathrm{z} / 2 \mathrm{r}_{\mathrm{o}}=0.5 \quad\left(\lambda_{\mathrm{j}}=5.1\right)$ 
Concentration spectra measured for the three flow configurations are shown in Figures 48 and 49 . In Figure 48, the laser "probe" is positioned at $x / 2 x_{0}=4$, $|y| / 2 r_{0}=0.5$ and $z / 2 r_{0}=0.5$. Consider first the case of the unconfined coflowing jet. Notice the relatively large peak in the spectra at approximately $300 \mathrm{~Hz}$. With the plate placed in the flow field, the peak is attenuated, and there also is an.increase in the energy at lower frequencies. By replacing the plate with the flap, this same trend is continued. The peak is diminished further and the energy at the lower frequencies are slightly increased.

In Figure 49, the concentration spectra for the jet and jet/plate flow are presented for the following downstream location: $x / 2 r_{0}=8,|y| / 2 r_{0}=0.5$ and $z / 2 r_{0}=$ 0.5. At eight diameters downstream, the peak in the spectra has disappeared. Here again, the presence of the plate in the flow field has caused an increase in energy of the concentration spectra at low frequencies.

Section 4.7 Space-Time Two Point Velocity Correlations The general space-time correlation coefficient for two points separated in a given flow field is as follows: 
(f)

(sec)
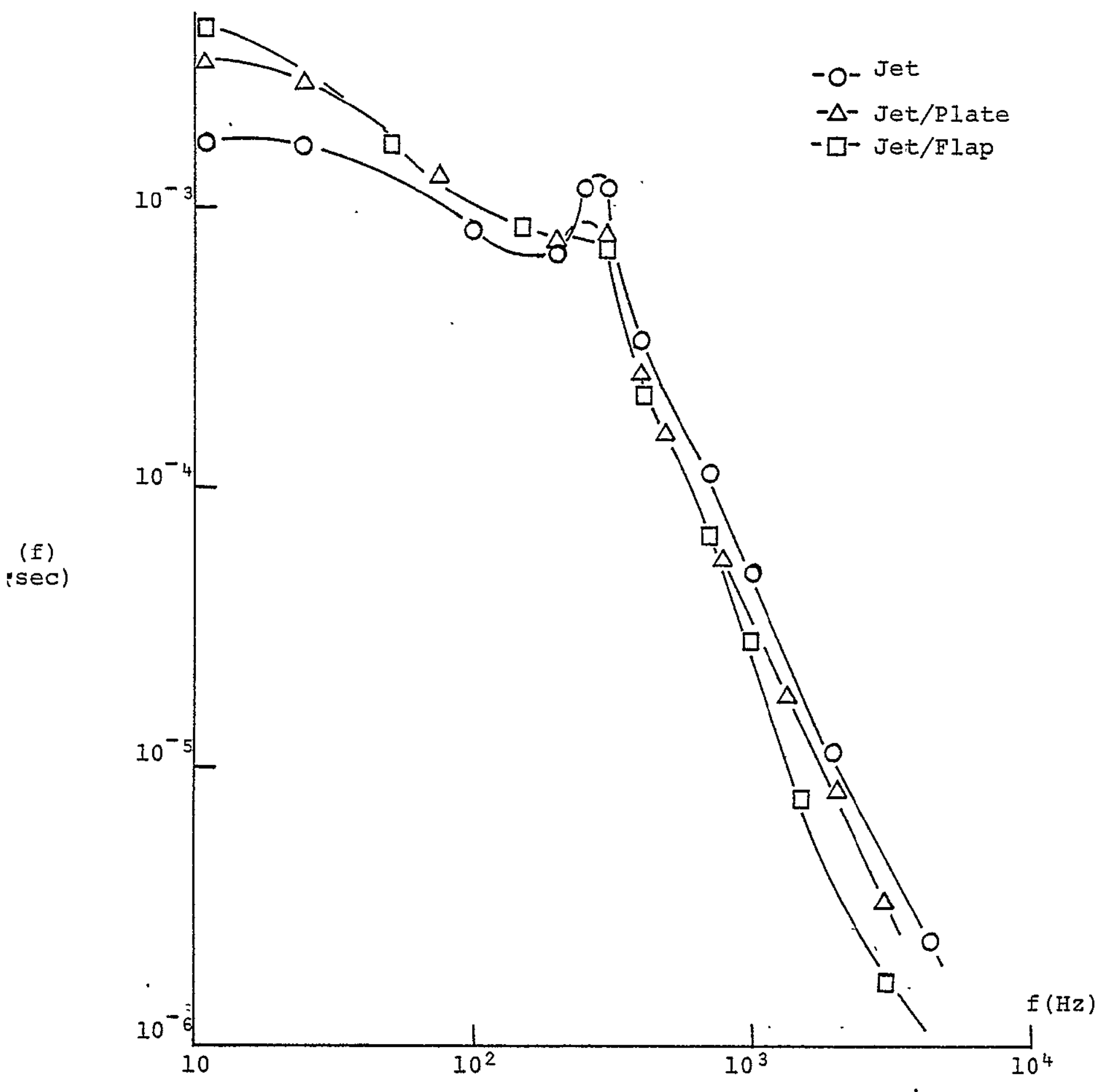

Figure 48 Concentration Spectra Comparison, $x / 2 x_{0}=4$, $|y| / 2 r_{0}=0.5, z / 2 r_{0}=0.5$

$$
\left(\lambda_{j}=5: 1\right)
$$




$$
R_{1}, 1\left(x, y, z, t, \xi_{1}, \xi_{2}, \xi_{3}, \tau\right)=
$$

$$
\frac{u(x, y, z, t) u^{\prime}\left(x+\xi_{1}, y+\xi_{2}, z+\xi_{3} ; t+\cdots \tau\right)}{\left\{\overline{\left.u^{2}\right\}^{\frac{3}{2}}}\right.}
$$

where $\xi_{1}{ }^{\prime} \xi_{2}$ ¿and $\xi_{3}$ represent the spatial displacements of one point relative to the other and $\tau$ represents the delay time. Here, the space-time correlations are limited in scope to fluctuating velocities in the $\mathrm{x}$ direction only. 'From these measurements, it is possible to calculate isocorrelation curves for optimum delay, $\tau_{m}$. Optimum delay time refers to the delay time at which for a given spatial separation the correlation is a maximum.

Typical results obtained are shown in Figures 50 thru 55. Figures 50 thru 52 deal with the case when $\xi_{2}$ is set equal to zero and hence are cross-sectional views of the three flow fields in the $x-z$ plane. Figures 53 thru 55 correspond to the isocorrelations curves obtained with $\xi_{1}$ set equal to zero and thus represent cross-sectional views in the $y^{-z}$ plane.

The reference point which remains fixed for all the correlation coefficients determined in the three flows has the following coordinates: 


$$
x / 2 r_{0}=4 \quad|y| / 2 r_{0}=0 \quad z / 2 r_{0}=0.5
$$

This downstream location is chosen for several reasons. First, this is the position at which the flap first begins to curve in a concave manner away from the x-direction. secondly, it has been observed in many of the one point measurements presented earlier in this report that there are significant differences in the three flows at this position, and also the statistical properties have been carefully documented.

Before progressing further, a new term must be introduced and it is the line of maximum maximorum of the correlations. (64) Physically what is meant is that a line is drawn through the point on each correlation level which is separated the farthest amount from the reference point (i.e. $\left(\xi_{1}{ }^{2}+\xi_{2}{ }^{2}\right)^{\frac{1}{2}}$ or $\left(\xi_{2}{ }^{2}+\xi_{3}\right)^{\frac{1}{2}}$ is a maximum). With this line, it is then possible to get an idea of the flow pattern.

In Figure 50, the isocorrelation curves in the $x-z$ plane for the case of the unconfined, coflowing jet are shown. The curves are very elongated in the streamwise direction and close to being symmetric with respect to the $\xi_{1}$ axis. This is not the case about the $\xi_{3}$ axis, which is in contrast with contours presented for a fully developed turbulent boundary layer. ${ }^{(65)}$ The difference 


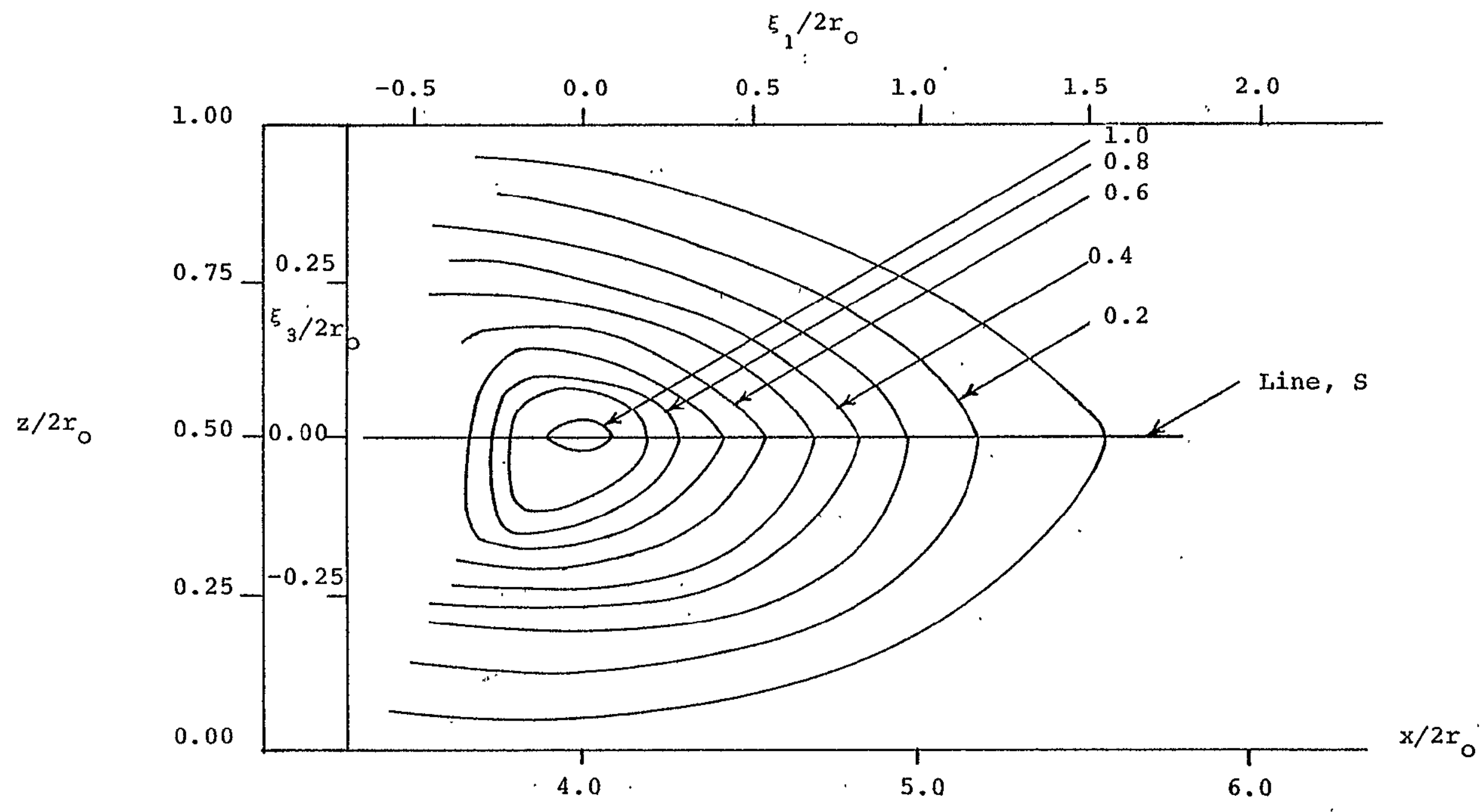

Figure 50 Isocorrelation Curves for Jet in $x-z$ Plane

$$
\left(\lambda_{j}=5.1\right)
$$


presumbly is due to the fact that part of the flow field lies in the potential core while the rest can be considered a mixing region. The line $s$, marking the points of maximum maximorum of the correlations, is approximately straight as expected.

The isocorrelation curves in the $x-z$ plane for the case of the turbulent jet flowing over the plate are shown in Figure 51. Once again, the curves are elongated in the streamwise direction. There quite clearly is not any axis of symmetry. The pattern formed by the isocorrelation curves is very complex and is composed partly of two different lobes of high correlation value levels. The line $S$ is drawn through the points of maximum maximorum of the correlations as was done in Figure 52. Downstream of the fixed reference point, the line is convex towards the surface of the wall. Upstream of the reference point, it is slightly more difficult to construct the Iine but it also seems to be convex towards the plate's surface. Sternburg ${ }^{(64)}$ has offered a possible explanation of a qualitative nature for the observed shape of the s-Iines. He theorizes that this may be caused by the mean shear, deforming the turbulence flow pattern as determined by its vorticity distribution. The maximum correlation will be determined by a point of the vorticity 


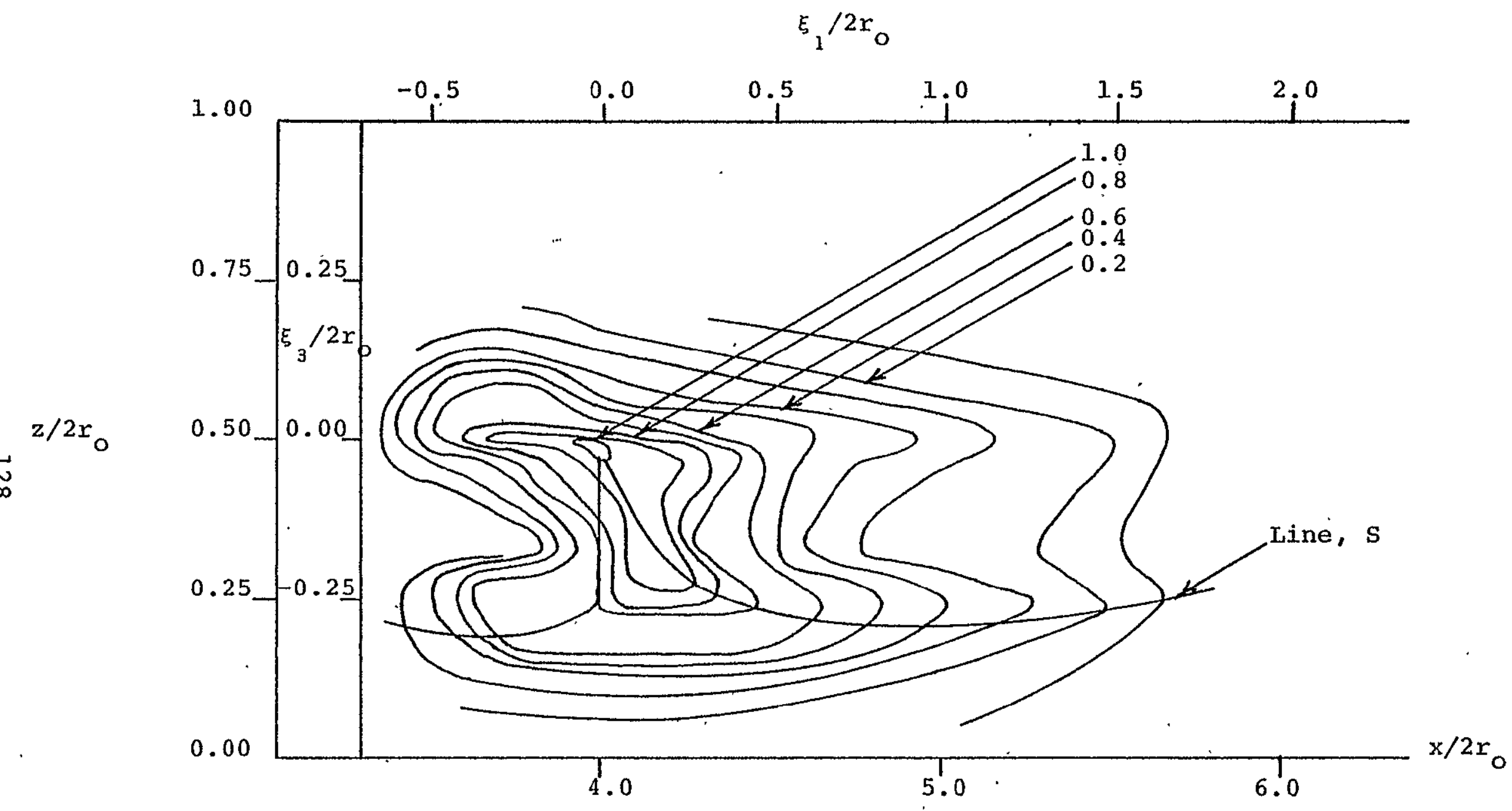

Figure 51 Isocorrelation Curves for Jet/Plate in $x-z$ Plane

$$
\left(\lambda_{j}=5.1\right)
$$


pattern that passes the fixed laser control volume at $\tau$ equal to zero and is observed at some time, $\tau$, later. The larger eddies which have a longer correlation distance will rotate due to the distortion of the mean shear flow in a clockwise sense and with a center of rotation in $\mathrm{x}-\mathrm{z}$ plane. As the second, movable control volume approaches the plate, a point of the flow pattern passing the fixed laser "probe" at $\tau=0$ will be displaced on the average from the wall by this rotation for $\tau>0$, while for $\tau<0$ this point has arrived from an upstream position also at a greater distance from the wall. The rough picture would lead to an S-line convex towards the wall below the center of rotation, a fairly straight line near the center and it will bu curved concave to the wall above the center.

The isocorrelation contours in the $x-z$ plane, for the flow of the turbulent jet over the flap are presented in Figure 52. As is the case for the plate configuration, the pattern formed by the isocorrelation contours is clearly not symmetric about either the $\xi_{1}$ or the ' $\xi_{3}$ axis. Before it has been observed that the previous two iso-contours demonstrated an elongation in the streamwise direction. For the flow over the flap, the elongation exists but the pattern formed is rotated a sizeable amount from the 


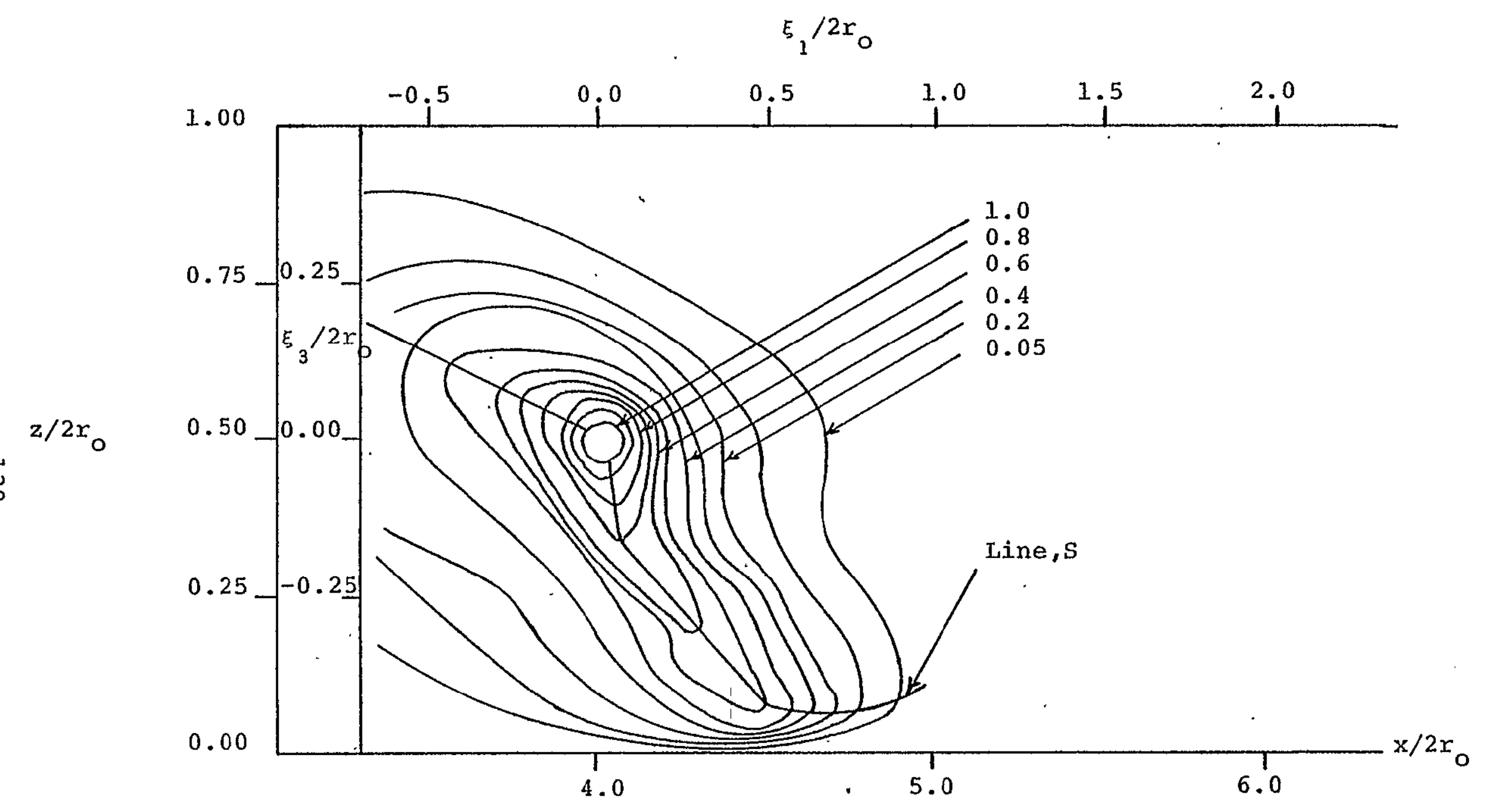

Figure 52 Isocorrelation Curves for Jet/Flap in $x-z$ Plane

$$
\left(\lambda_{j}=5.1\right)
$$


longitudinal direction. Through the points of maximum maximorum of the correlations, the line $s$ is drawn. Before applying sterburg's approach, it is first necessary to recall the geometry of the flap and the relation position of the reference "probe". In Figure $7(a)$, a view of the jet/flap assembly is shown. After a distance of approximateIy four diameters downstream from the exit plane of the nozzle, the tangent to the surface of the flap rotates quickIy away from the horizontal direction. Thus, any position for the second control. volume downstream of the $x / 2 x_{0}=4$ plane would be over the curved section of the flap's surface. Now, consider the shape of line $s$ in Figure 47. Downstream of the reference point, the line heads down towards the wall surface and is also convex to the flap. Upstream the line is also inclined with a negative slope from the $x$-direction, and is essentially straight. clearly, it is possible to say from observing the isocorrelation contours that the flow is being turned by the flap. Secondly, considering the change in shape of the flap surface and the convex shape of the line S downstream, Sternburg's ideas can be reasonably applied once again. For the clockwise rotation of the large eddies, a point of the flow pattern passing the reference control volume will tend to both be turned downward by the flap's surface and also be displaced on the average from the wall 
by this rotation for a positive delay time. The flow pattern upstream of the fixed reference point indicates the direction from which the flow is coming.

In Figure 53, the isocorrelation curves in the $\mathrm{y}^{-\mathrm{z}}$ plane for the unconfined coflowing turbulent jet flow field are shown. The eccentricity of each of the curves does vary but is essentially zero and thus the isocorrelation levels are close to being circles in shape. Hence, symmetry exists about both the $\xi_{2}$ and the $\xi_{3}$ axes. Though Sternburg's ideas cannot be applied in the $y^{-z}$ plane, the line $S$ can be constructed and from its shape, a rough estimate of the flow pattern can be obtained. For the unconfined jet, the line of maximum maximorum can be drawn, starting from the center of field, off in any radial direction. One line is arbitrarily constructed for the sake of clarity. From observing the line $S$ and the isocurves, an indication that the jet's flow field is spreading out equally in all directions is apparent. The lower correlation. levels farther out from the center line represent fluid particles which have been separated from the potential core the longest period of time.

Consider next the isocorrelation curves in the $y-z$ plane for the flow of the turbulent jet over the plate Figure 54. The change in the shape of the curves caused 


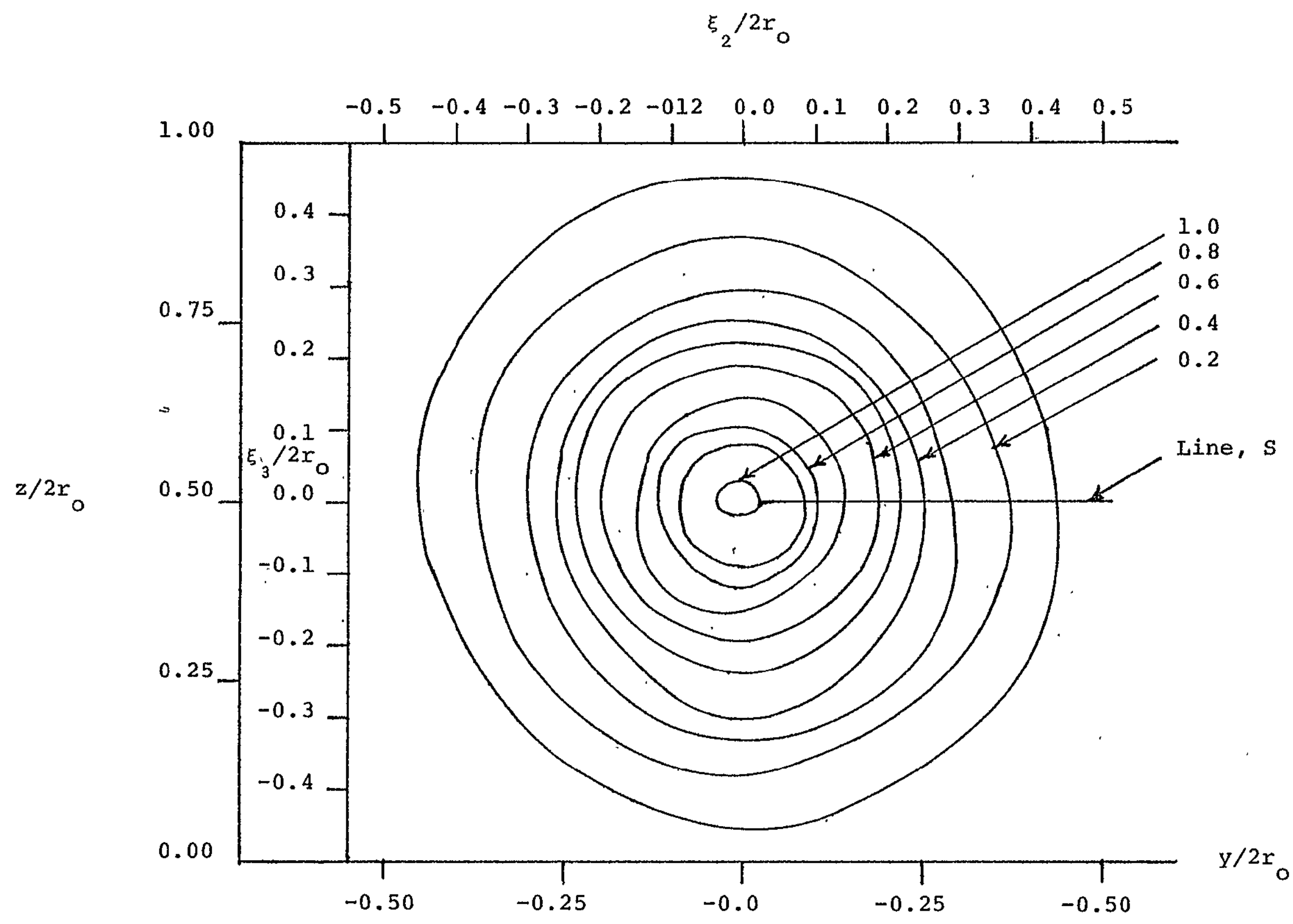

Figure 53 Isocorrelation Curves for Jet in $y^{-z}$ Plane

$$
\left(\lambda_{j}=5.1\right)
$$




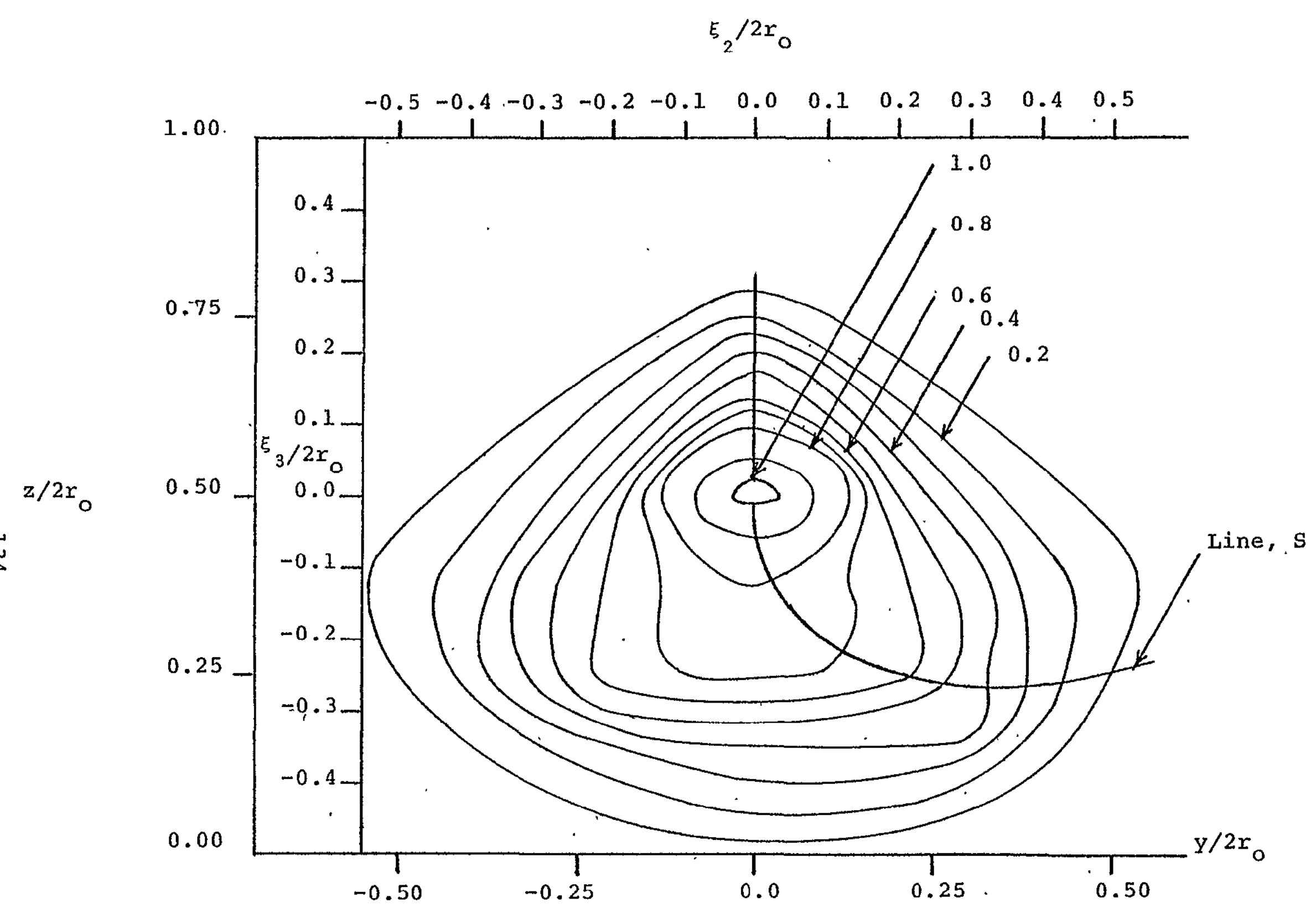

Figure 54 Isocorrelation Curves for Jet/Plate in $\mathrm{y}^{-\mathrm{z}}$ Plane

$$
\left(\lambda_{j}=5.1\right)
$$


by the presence of the plate is apparent. The isocorrelation curves are elongated in the lateral direction. Although the flow pattern is clearly not symmetric about the $\xi_{2}$ axis, symmetry does exist with respect to the $\xi_{3}$ axis. As was the case in the $x-z$ plane, the line $S$ is again somewhat more difficult to construct. Allowing some author's discretion, the line $s$ moves out from the center of the flow in a slightly convex manner with respect to the plate's surface. Two impressions can be drawn. Initially, the turbulent jet is drawn downwards towards the plate. Secondly, there exists an indication that the flow may be rolling up in the outer regions, or at least some type of canted rotating motion of the larger eddies to the longitudinal downstream is being observed. It is instructive to compare the differing flow patterns displayed in Figures 54 and 55. The width of area enclosed by the isocorrelation curves is significantly smaller for the flow over the plate for $\xi_{3}>0$, about the same for $\xi_{3}=0$ and somewhat larger for the $\xi_{3}<0$. The second observation is reinforced by the measurements made of the mixing widths and the intermittency profiles for the two flows.

Iastly, the isocorrelation curves in the $y-z$ plane for the flow of the turbulent jet over the flap are shown in Figure 55. The' isocorrelation curves are markedly 
elongated in the $z$ direction. The flow pattern is symmetric about the $\xi_{3}$ axis but asymetric with respect to the ${ }^{\prime} \xi_{2}$ axis. The line $s$ is drawn appropriately through the flow field pattern. The first impression is one of noticing the narrowness of the flow pattern. As has been mentioned previously, the flap is much more effective in amplifying the turbulence levels of the turbulent jet. This more rapid and intense mixing action would manifest itself in a decrease in the coherence of the flow field pattern. Thus, in the outer mixing region, the turbulent jet is more apt to have forgotten its previous flow conditons, and correlations between the center of field and a second point farther out in the mixing layer would be weaker. Secondly, consider the direction of the line $s$. Downstream of the fixed reference point, the line is clearly heading in a negative $\xi_{3}$ (i.e. - z) direction. Upstream, there is some arbitrariness involved but the line also has a negative $\xi_{3}$ heading. From this flow pattern the impression is made again that flap is quite effective in turning the flow. At a positive delay time, the particles that were at the reference point have moved steadily downwards towards the surface of the flap, and thus a stronger correlation level exists. The same type of agreement can also be made for the flow upstream of the reference point. 
Section 4.8 Convection Velocities

The possibility exists of defining many kinds (65)

of convection velocities. Favre, Gaviglio and Dumas (66) showed that convection velocities can be determined from iso-space-time velocity correlation curves $R_{1}^{\prime}(\xi, 0,0, T)$. Favre et. al. then defined the convection velocity as follows:

$$
\text { Ucon }=\xi_{1} / \tau_{\max }
$$

where $\tau_{\max }$ is the delay time for which $R_{1}{ }^{\prime}$, has a maximum value.

In particular in the case of relatively strong inhomogeniety taking in the dimensionless time $\bar{U} t / L$, an average value of $\overline{\mathrm{U}}$ across the distance $\xi_{1}$, i.e.

$$
\overline{\mathrm{U}}_{1}=\frac{1}{\xi_{1}} \int_{0}^{\xi_{1}} \mathrm{~d}_{1} \overline{\mathrm{U}}_{1}\left(\mathrm{x}_{1}+\xi_{1}\right)=\frac{1}{2}\left[\overline{\mathrm{U}}_{1}\left(\mathrm{x}_{1}\right)+\overline{\mathrm{U}}_{1}\left(\mathrm{x}+\xi_{1}\right)\right] \text {. }
$$

where $I$ is any suitable length scale.

The convection velocities determined for the three aifferent flow configurations are shown in Table 2. Here for a given longitudinal displacement, nondimensionalized by twice the radius of the jet, between the two measuring laser "probes", the non-dimensionalized delay time and the ratio of the convection velocity to the mean velocity are given. Mean velocity here refers to the average 
TABIE 2

Convection Velocities in Various Flows

Coflowing Jet

\begin{tabular}{|c|c|c|}
\hline$\frac{\xi_{1}}{2 x_{0}}$ & $\frac{\bar{U}_{1} T}{2 x_{0}}$ & $\frac{U \operatorname{con}}{\overline{\mathrm{U}}_{I}}$ \\
\hline $\begin{array}{c}-.752 \\
-.595 \\
0 \\
.177 \\
.610 \\
.942 \\
1.22 \\
1.59 \\
1.82- \\
2.73\end{array}$ & $\begin{array}{c}-.561 \\
-.527 \\
0 \\
.210 \\
.606 \\
.921 \\
1.17 \\
1.51 \\
1.65 \\
2.49\end{array}$ & $\begin{array}{r}1.34 \\
1.12 \\
1 \\
.843 \\
1.01 \\
1.02 \\
1.04 \\
1.05 \\
1.10 \\
1.09\end{array}$ \\
\hline
\end{tabular}

Coflowing Jet/Plate

\begin{tabular}{|c|c|c|}
\hline$\frac{\xi_{1}}{2 r_{0}}$ & $\frac{\bar{U}}{2 r_{0}}$ & $\frac{U \text { con }}{\bar{U}_{1}}$ \\
\hline $\begin{array}{c}-.761 \\
-.595 \\
0 \\
.262 \\
.476 \\
1.14 \\
1.42 \\
2.23 \\
3.06 \\
5.09\end{array}$ & $\begin{array}{c}-.786 \\
-.587 \\
0 \\
.262 \\
.393 \\
1.05 \\
1.28 \\
1.79 \\
2.42 \\
3.07\end{array}$ & $\begin{array}{l}.968 \\
1.01 \\
1 \\
1 \\
1.21 \\
1.08 \\
1.11 \\
1.25 \\
1.26 \\
1.66\end{array}$ \\
\hline
\end{tabular}

\begin{tabular}{c|c|c}
$\xi_{1}$ & $\bar{U} I_{1}$ & $\frac{\text { Ucon }}{\bar{U}_{0}}$ \\
\hline & $\frac{1}{2 r_{0}}$ & $\bar{U}_{1}$ \\
\hline-1.49 & 1.28 & 1.16 \\
-1.03 & -.925 & 1.11 \\
-.480 & -.40 & 1.20 \\
0 & 0 & $I$ \\
.426 & 1.76 & .242 \\
1.770 & 2.98 & .258 \\
1.14 & 3.83 & .297 \\
1.86 & 6.26 & .297 \\
2.55 & 8.50 & .300
\end{tabular}


value determined using Favre's definition.

Focussing initially on the convection velocity calculated for the unconfined, coflowing turbulent jet, the ratio of the Ucon to $\bar{U}_{i}$ is very close to unity, and, indeed, remains fairly constant. Thus, in this case, at least, the convection velocity appears to be almost independent of the separation distance. Next, consider the case of the turbulent jet flowing over the plate. Here someth ng quite interesting happens. As the flow progresses downstream, the convection velocity has approximately the same value as the mean velocity for $\xi_{1} / 2 x_{0} \leq 0$ but for $\xi_{1} / 2 x_{0}$ increasing positively, the ratio Ucon $/ \bar{U}_{1}$ is greater than unity. This phenomenon also occurs in turbulent boundary layers over flat plates. ${ }^{(65)}$ To explain the reason for this behavior, recall the vertical height above the surface of the plate at which these measurements are taken and also the shape of the mean velocity profile for this flow in the center vertical plane. Notice that there is a region of faster moving fluid particles closer to the wall surface. Also, as the flow progresses downstream, the profile approaches that found for a classical wall jet. Hence though the ratio of Ucon $/ \bar{U}_{1}$ may be greater than unity, the ratio of Uc/U max will never be. 
Finally, consider the flow of the turbulent jet over the flap. Notice that for $\xi_{1} / 2 r_{0}<0$, the value of the ratio of Ucon $/ \overline{\mathrm{U}}{ }_{1}$ is slightly greater than unity. This can be explained using the same logic that is used in the previous paragraph. The reason that Ucon/U $U_{1}>I$ occurs sooner than is the case for the flow over the plate is that the mean velocity profile over the curved surface in the center vertical plane is transformed much more effectively and rapidiy from the ideal jet's "flat top" to the wall jet shape. For $\xi_{1} / 2 x_{0}>0$, the convection velocity has decreased substantially. presumably this is due to the fact that the flow is undergoing a change in direction.

Section 4.9 Pressure-Velocity Correlations

Additional information concerning the turbulence structure of the various flow fields can be gained from measurements of the pressure fluctuations at both the wall and in the turbulent jet and correlating those signals with the fluctuating turbulent velocities in the potential core and in the shearing region..

With the orgin of the coordinate system at the location of either the surface port or the pressure probe, the follwoing space-time correlations are measured: 


$$
\mathrm{R}_{\mathrm{pj}},\left(x, y, z, t, \xi_{1}, \xi_{2}, \xi_{3}, t\right)=\frac{\overline{p(0,0, t) u\left(\xi_{2}, \xi_{3}, t+t\right)}}{\overline{\left[p(0,0 ; t)^{2}\right]^{\frac{1}{2}}\left[\overline{\left(\xi_{2}, \xi_{3} ; t+t\right)^{2}}\right]^{\frac{1}{2}}}}
$$

where $p$ is the static pressure measured at the waII or in the flow field.

Since the primary focus of this segment of the investigation is to determine the relationship between the pressure and velocity fields, the coherence is plotted for various pressure and velocity monitoring locations. Recall that coherence is between two signals defined as follows:

$$
\delta_{12} 2=\frac{\left.G_{12}\right|^{2}}{G_{11} G_{22}}
$$

where $G_{11}, G_{22}$ are the Fourier Transforms of the individual autocorrelation functions and $G_{12}$ is the Fourier Transform of the cross correlation function.

Case I Wall Pressure Fluctuations

Cross correlations between fluctuating pressure signals measured at surface ports in the plate and flap and turbulent velocity signals monitored at various locations in the flow field are determined. For the flat plate case, coherences are determined with the laser probe at $\xi_{3} / 2 r_{0}=0.2$ with $\xi_{1} / 2 r_{0}$ and $\xi_{2} / 2 r_{0}$ set equal 
to zero. Examples of the results for $x / 2 x_{0}=4$ are shown in Figure 56. Here the lateral positioning of the pressure port and laser control volume varies from $|y| / 2 r_{0}=$ 0 to $|y| / 2 r_{0}=1$. It is found that inside the lip of the nozzle, the velocity spectra and pressure spectra are characterized by large peaks at, in this instance, approximately $300 \mathrm{~Hz}$. The coherence between the pressure and velocity signals is clearly the strongest at this peak frequency. As the pressure and velocity monitoring "probes" are moved outward from $|y| / 2 r_{0}=0$, the spectral peaks are attenuated and the resultant decrease in the coherence is noted in Figure 56.

Coherences are also determined for the flow over the plate with $\xi_{2} / 2 x_{0}$ varying from 0 to $1, \xi_{1} / 2 x_{0}$ held equal to zero and $\xi_{3} / 2 r_{0}$ set equal to 0.5 (Figure 57). Physically, the laser control volume is held fixed at the location $x / 2 r_{0}=4,|y| / 2 r_{0}=0$, and $z / 2 r_{0}=$ 0.5 with the fluctuating velocity signal being correlated with surface pressures measured at $x / 2 r_{0}=4, z / 2 r_{0}=0$ and $|y| / 2 r_{0}=0,0.5$ and 1 . It is found that the velocity spectra is not as peaked as is the case at $z / 2 r_{0}=0.2$ and, hence, the coherence between the pressure and. velocity signals is diminished. Also, the coherence decreases as the surface probe is moved laterally outward from the jet centerline. 


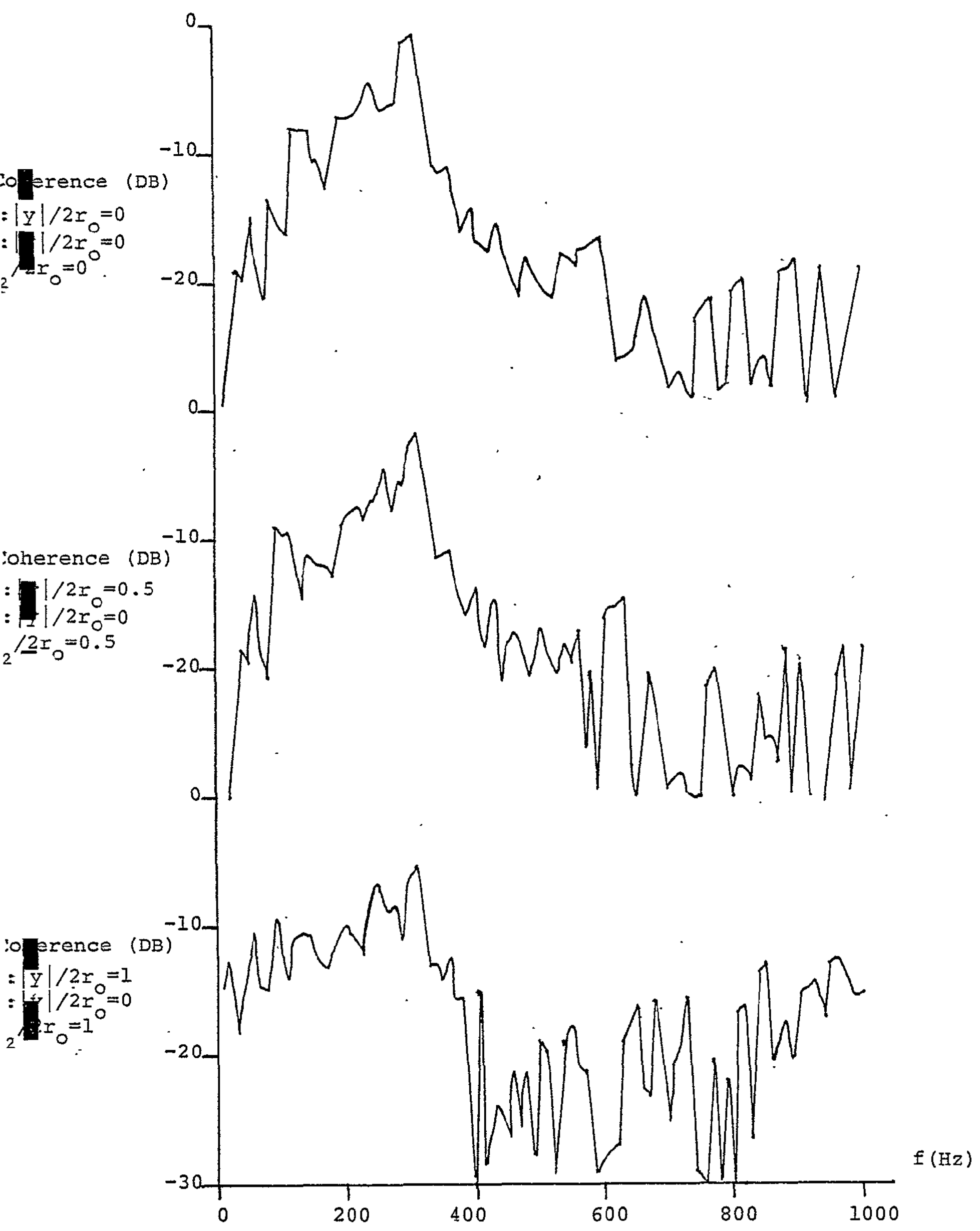

Figure 57 Wall Pressure and Velocity Coherence. $\xi_{/} / 2 r_{0}=0$. $\xi_{\text {Píns }} / 2 x_{0}=0.5\left(\lambda_{j}=5.1, D B\right.$ referenced tô Dynamic 
For the flow over the flap, coherences are determined between the pressures monitored at the surface and the velocities at the $|y| / 2 r_{0}=0$ location.' The downstream location is held fixed at $x / 2 x_{0}=4$. In Figure 58 , coherences are shown with the laser "probe" held fixed at $|\dot{y}| / 2 r_{0}=0$ and $z / 2 r_{0}=0.2$. while in.Figure. 59 , the laser measuring volume is located at $|y| / 2 r_{0}=0$ and $z / 2 r_{0}=0.5$. Many of the' same observations that were made concerning the flow over the plate can be made once again. The velocity spectra is found to be much more peaked closer to the surface of plate. The coherence is larger in magnitude between the wall surface pressure and the velocity fluctuations with $\xi_{3} / 2 r_{0}=0.2$. Also, the coherence is the strongest at approximately $300 \mathrm{~Hz}$.

Case II Pressure Fluctuations at Probe

Cross correlations and coherences are determined between the static pressure measured by a static pressure probe and the turbulent velocity fluctuations measured at the jet axis. The pressure is monitored at three lateral locations, (i.e. $t y \mid / 2 r_{0}=0,0.5,1.0$ ) with the velocity control volume remaining fixed. The vertical location for the pressure probe and IDV volume remains fixed at $z / 2 r_{0}=0.5$.

Figure 60 shows the change in the value of the correlation coefficient at a given lateral location as 
.

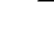



$R_{p, u}$
$P:|y| / 2 r_{0}=0$

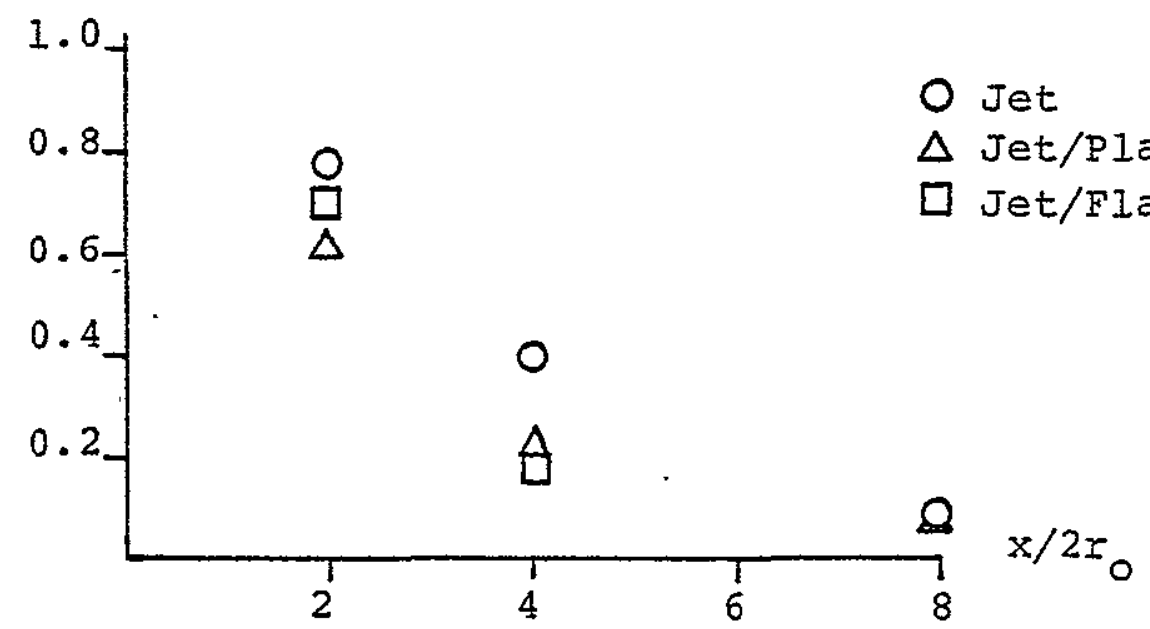

$p:|y| / 2 r_{0}=0.5$.

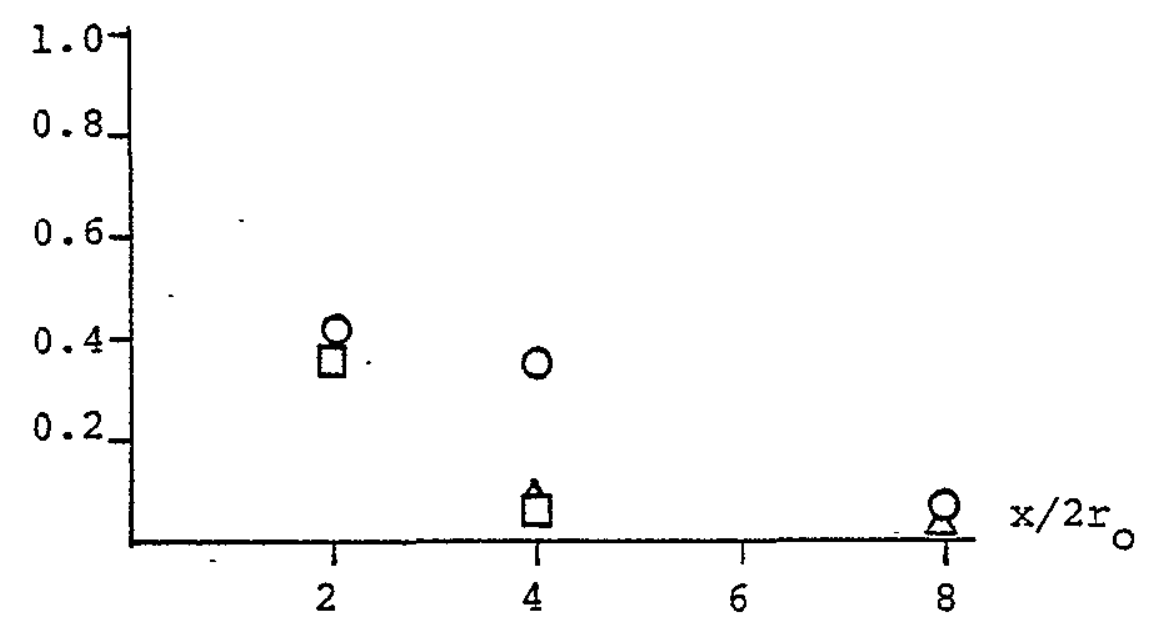

$p: \begin{gathered}R_{p, u} \\ p|y| / 2 x_{0}=I\end{gathered}$

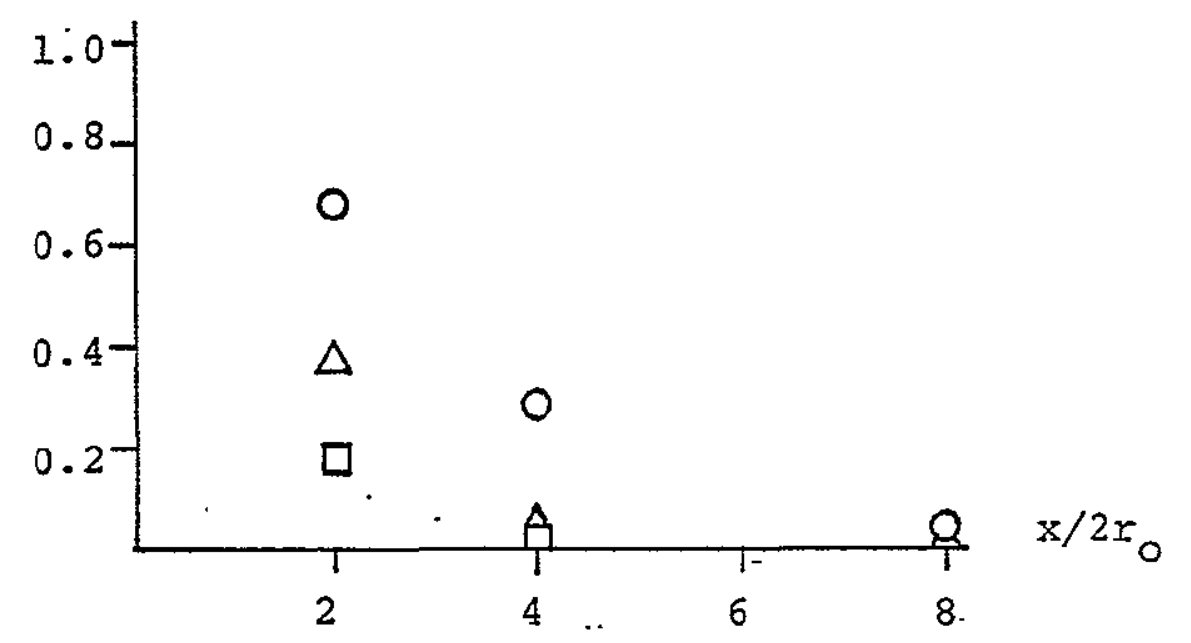

Figure 60 Lateral Displacement of Pressure Probe Effects on pa Coefficient $\quad\left(\lambda_{j}=5.1\right)$ 
the flow progresses downstream in each of the three configurations. The decrease in the correlation coefficient as $x / 2 x_{0}$ increases is apparent. Also, the flow of the unconfined, coflowing turbulent jet maintains the highest value of the coefficient at each measurement position. For the unconfined, coflowing jet, the coherences between the fluctuating pressure and velocity signals are shown in Figure 61 for the downstream location, $x / 2 r_{0}=4$. It is found that both the pressure and velocity spectra are characterized by large peaks at approximately $300 \mathrm{~Hz}$. This clearly is the frequency for which the coherence is. a maximum. As is evidenced in the case of wall pressure fluctuations, the coherence between the pressure and velocity signals decreases as the pressure probe is moved lateraliy outward.

For purposes of comparison, the pressure and velocity spectra, and coherence at $x / 2 r_{0}=8$ at the jet axis.are also shown in Figure 62 . The peak which charactexizes both the pressure and velocity spectra is absent and the coherence has been reduced significantly. At this distance downstream from the exit plane, the turbulence has become much more fully developed.

Next, consider the case of the turbulent jet flowing over the flat plate for the downstream location $\mathrm{x} / 2 \mathrm{r}_{0}=4$ (Figure 63 ). The pressure spectra is again 


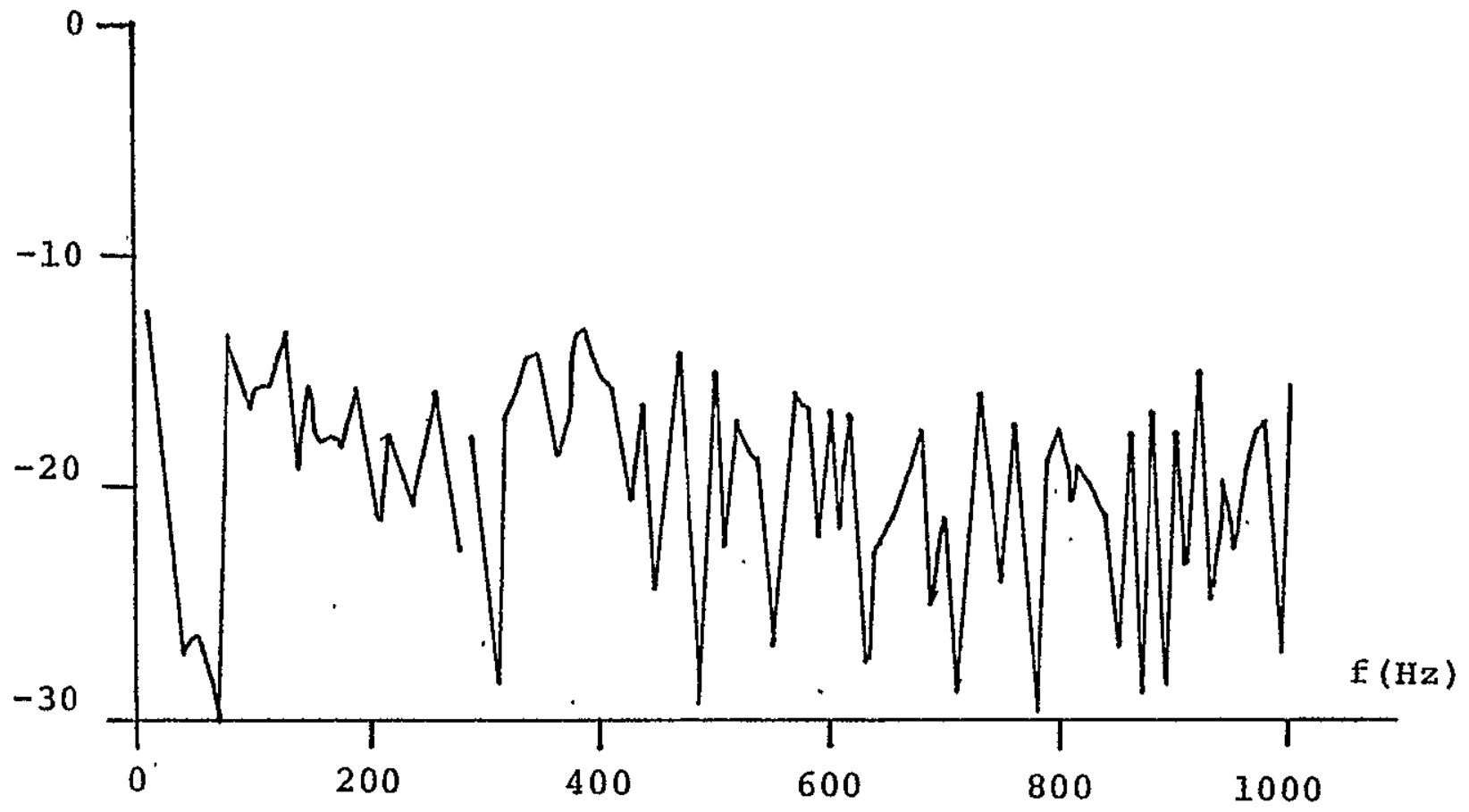

Figure 62 Static Pressure and Velocity Coherence. $\xi_{1} / 2 r_{0}=0$. $\xi_{3} / 2 r_{0}=0\left(\lambda_{j}=5.1, D B\right.$ referenced to Dynamic Pressure $)$ 


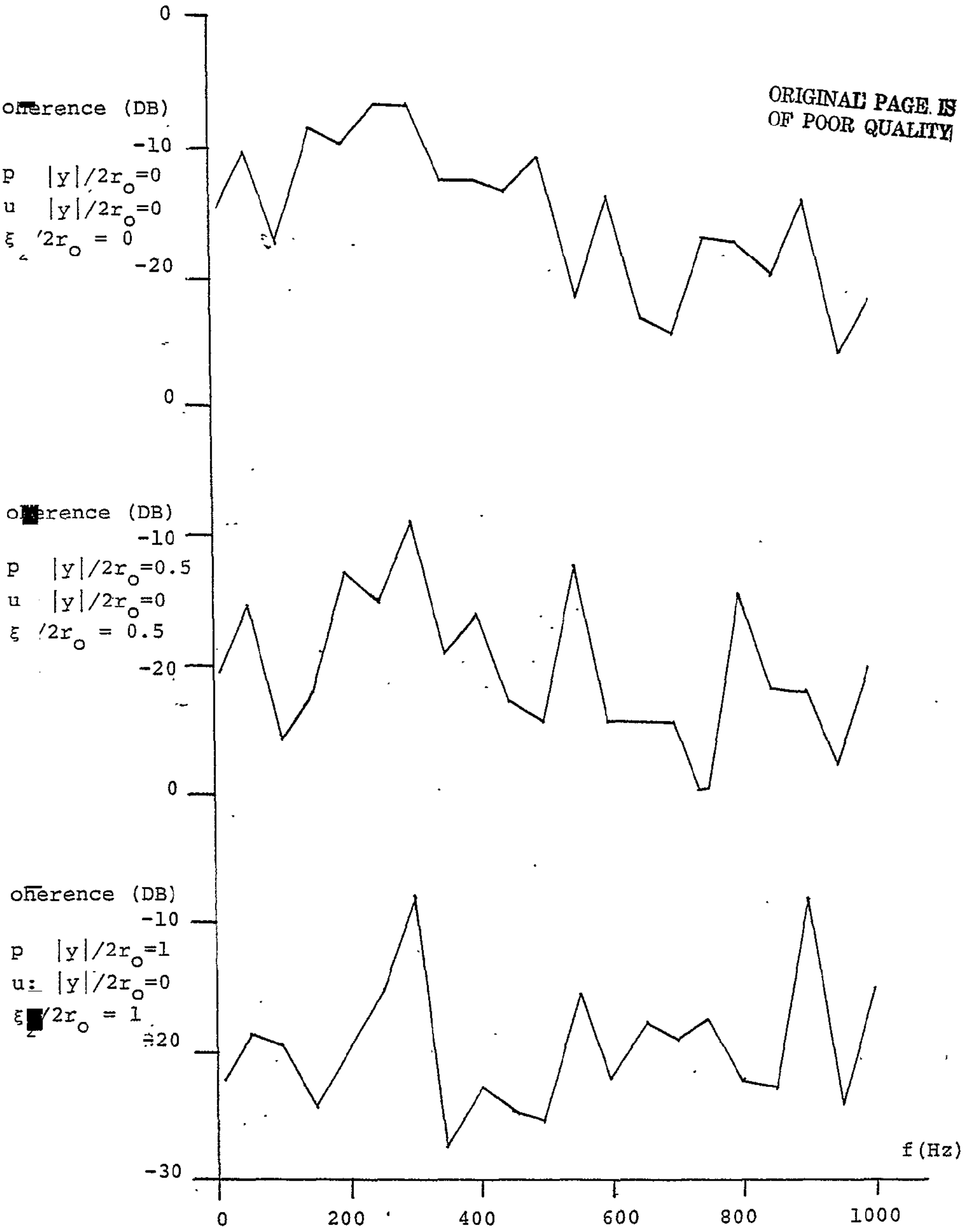

Figure 63 static Pressure and Velocity Coherence. $\xi_{1} / 2 r_{0}=0$, $\xi_{3} / 2 r_{0}=0 . \quad\left(\lambda_{j}=5.1, D B\right.$ referenced to Dynamic Pressure $)$ 


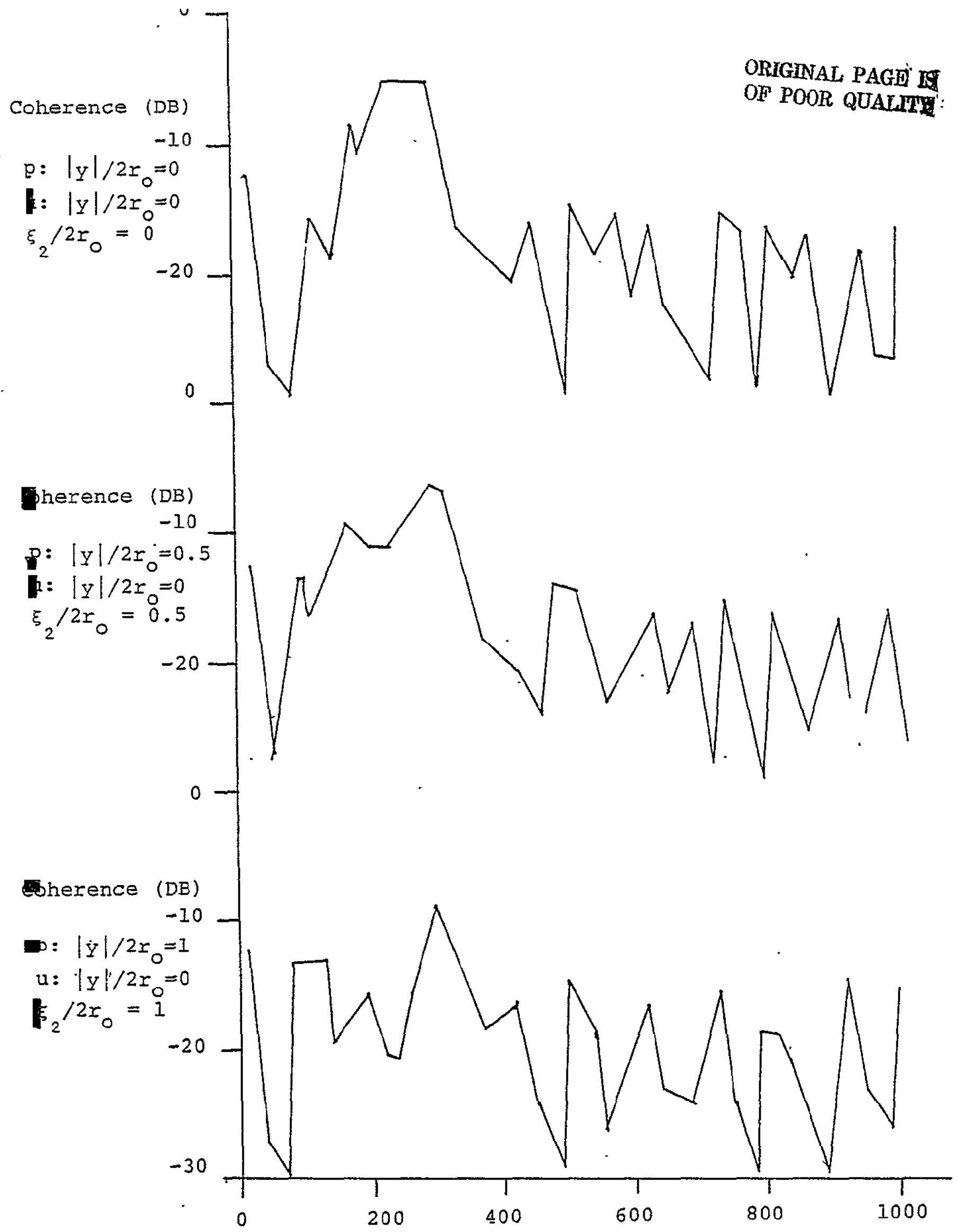

Figure 64 static Pressure and Velocity Coherence. $\xi_{1} / 2 r_{0}=0$, $\xi_{3} / 2 x_{0}=0\left(\lambda_{j}=5.1, D B\right.$ referenced to Dynamic Pressure $)$ 
found to be markedly peaked at $300 \mathrm{~Hz}$ at $|\mathrm{y}| / 2 \mathrm{x}_{0}=0$ and this "bump" is both broadened and attenuated out from the jet axis. The velocity spectra though not peaked as it is in the case of the unconfined jet is not at all similar to the fully turbulent example at $x / 2 r_{0}=8$. The coherence of the two fluctuating signals has been significantly reduced with the plate in the flow field, and it decreases with increasing lateral separation of the pressure probe and laser control volume. The coherence obtained from the flow field of the turbulent jet and flap at the downstream location $\mathrm{x} / 2 \mathrm{r}_{\mathrm{o}}=4$ are shown in Figure 64 . The peak in the velocity spectra has been virtually eliminated. It, is interesting to compare the coherence of each. $\xi_{2} / 2 x_{0}$ position for this flow with the jet/plate and unconfined jet flow fields. The coherence for the two confined jet flows are quite close in magnitude. The transformation of the turbulent jet into-either a classical wall jet or curved wall jet does decrease the relationship between the fluctuating pressure and velocity fields. 


\section{Chapter 5}

\section{SUMMARY}

A sumary of the significant results detailed in this experiment will now be given. The presence of the confining surfaces was found to be of importance in the resultant effects on both the mean and fluctuating part of the turbulent velocity field of the jet.

(1) The mean velocity was measured for each of the three flow configurations at the jet axis $\left(z / 2 r_{0}=0.5\right)$ at different downstream locations. The decay of the mean velocity of the turbulent jet was accelerated by the presence of the confining surfaces. The Elap was also found to be the most effective in diminishing the $\mathrm{x}-$ directed momentum. For an increasing value of $\lambda_{j}$, the effectiveness of the flap in diminishing the $x$-directed momentum decreased. Evidence existed that as $\lambda_{j}$ approached infinity, the flow would not remain attached.

(2) The flap seemed to break up the top hat characteristic shape of the mean velocity profile at the exit plane much more rapidly. The width of the flow field increased at a faster rate for the flow over the flap. The parameter $\lambda_{j}$ also influenced the width of the mean velocity profiles. For the case of the turbulent jet/flap, an increase in $\lambda_{j}$ caused a resultant decrease 
in the mixing width, $y_{m}$.

(3) It was found that for the flow over the plate, the mean velocity field tended toward the fully developed turbulent shape in different horizontal planes faster than in the vertical plane at $y / 2 r_{0}=0$. Also, the flap seemed more effective in changing the flat velocity profile characteristic of a circular nozzle into a wall jet profile.

(4) The confining surfaces caused a more rapid increase in the turbulent intensities in the $x$-direction at the jet axis. Here, as well, the value of $\lambda_{j}$ was also found to be important. At the large value of $\lambda_{j}$ ' the turbulent intensities for the flow over the flap increased at a significantly less rapid pace than was the case for $\lambda_{j}$ equal to 5.1. An indication that the flap and the plate serve to break up the potential core more rapidly was also noted and discussed.

(5) Turbulent intensities in the lateral, $y$, direction for the three flow configurations were also measured. The turbulent intensity was found to be much greater in the shearing layer of the unconfined jet than in either of the other two configurations. Some evidence thus exists which.indicate a damping of the turbulence in the $y$ direction by the presence of the confining surfaces. 
(6) Autocorrelations of the longitudinal velocity fluctuations were taken at the jet axis and at the lateral position corresponding to the lip of the nozzle at various $x / 2 x_{0}$ locations. The autocorrelations taken in the potential. core regions were characterized by a damped sinusoidal nature. The dominant frequency in the autocorrelation curve is theorized to be due to the shedding of vortices (i.e. large scale structures) at the exit plane of the nozzle. As the vortices pass downstream, the associated fluctuating pressure field causes velocity fluctuations which are monitored. The effects of the plate and especially the flap on the autocorrelation were apparent, causing a shift and attenuation of the frequency at $z / 2 x_{0}=4$. The changes in the autocorrelation curves as the flow progresses downstream are also noted. In the mixing layers of the flow fields, the dominant frequency shape was absent due to the large increase in the turbulent level and it was very difficult to determine any effects caused by presence of the confining surfaces.

(7) Longitudinal integral length scales and Taylor microscales were determined. The integral scales for the flap were seen to have a rapid increase and then decrease in magnitude as the flow approached the start of the curved portion of the surface. For the unconfined turbulent jet, the growth of the integral scale at $|y| / 2 x_{0}$ was linear, as 
predicted. At best, the integral scale development for the three different configurations was a weak function of $\lambda_{j}$. The value of $\lambda_{j}$ did strongly influence the magnitude of the Taylor microscale, $\lambda_{T^{\prime}}$ at the lateral position corresponding to the lip of the nozzle.

(8) Power spectra for the different confining surfaces reflected the change observed in the autocorrelation curves. At $x / 2 r_{0}=4$, the flows of the unconfined jet and jet/plate were still peaked in nature at the jet axis but for the flow over the flap, this "bump" was absent. The "bump" or peak in the power spectra corresponds to the passing downstream of the large scale vortex rings. As the flow becomes more fully developed due either to the amplification of the turbulence by the confining walls or simply the flow progressing downstream, the relatively small velocity fluctuations due to the shedaing vortices become less apparent and essentially become "lost" in the increased turbulent levels. It was interesting to note that with regard to the flap configuration, the absence of the peak was true for $\lambda_{j}=$ 5.1 and $\lambda_{j}=10.88$. This is inspite of the fact that for $\lambda_{j}=10.88$, the flow had not decelerated as much nor had there been as large an increase in the turbulent intensities in the central part of the velocity field. The downstream development of the power spectral densities at- the jet axis, $|y| / 2 r_{0}=0$, and out at the Iip of the nozzle, $|y| / 2 r_{0}=0.5$, 
were also presented. At the lateral position downstream from the lip of the nozzle, the power spectra consistently increased the low frequency content of the spectra as the flow progressed downstiream.

(9) Auto and cross correlations were determined for the concentration field at various downstream locations. At $x / 2 x_{0}=4$, the autocorrelation coefficient curve was found to possess a damped sinusoidal nature for the three different flow configurations. The concentration fluctuations at $y= \pm 0.5$ were found to be related both at $x / 2 r_{0}=4$ and $x / 2 r_{0}=8$. The maximum absolute values of the cross correlation coefficient did decrease sizably from four to eight diameters downstream of the exit plane, however. The power spectra for the jet, jet/plate, and jet flap all exhibited a peak at $x / 2 r_{0}=4$ at approximately the same-frequency as that found in the core velocity spectra. Here again, evidence exists in reinforcement of the vortex model of the jet. The plate and the flap did seem to diminish the peak and also seemed to cause a shift of energy to lower frequencies in the spectra.

(10) Space-time correlations for the turbulent velocity fields were obtained, and the Iines of maximum maximorum were constructed for both the $x-z$ plane and the $y-z$ plane. For the unconfined jet, the iso-correlation levels were symmetric with respect to the $\xi_{1}$ axis though very elongated. 
The line of maximum maximorum was straight and parallel to the $\xi_{1}$ axis. In the $y^{-z}$ plane, the isocorrelation levels were circular in shape about the $\xi_{1}$ axis. For the flow over the plate, the isocorrelation curves in the $x-z$ plane were very complicated and elongated in the $\xi_{1}$ direction and the line of maximum maximorum was curved convex towards the wall both upstream and downstream of the fixed reference "probe". For the flow over the flap the isocorrelation curves were elongated and rotated down towards the surface in the $x-z$ plane, evidence also existed as to confirm the effectiveness of the flap in turning the flow. The isocorrelation contours for the turbulent flow fields demonstrate the existence of large scale structures. Consider the fact that the shape of the contours is significantly different for each of the three flow configurations in both the $x-z$ and the $y-z$ planes. The flow "knows" whether or not a confining surface is present and also "senses" if the wall is curved or flat. This "knowledge" is then seemingly transmitted throughout the flow field.

(II) Convection velocities were determined for the three flow configurations. The convection velocity determined for the unconfined jei was found to be approximately equal to the local mean velocity and also to be independent of the separation distance between the "probes". For the cases of the flows over the plate and flap, the convection 
velocity was greater than the local mean velocity in several instances when the local velocity was considerably less than the maximum velocity at that particular downstream location.

(12) Pressure velocity correlations using both the static pressure probe and the surface ports yielded strong evidence that as the flow progresses, downstream, and the flow becomes a fully developed turbulent flow, the relationship between the pressure and velocity fields diminishes. For the first several diameters downstream from the exit plane when the pressure and velocity spectra peak at approximately $300 \mathrm{~Hz}$, the coherence between the two fluctuating fields is the strongest.

This experimental investigation has shown that large scale structures do exist in the near field of the turbulent jet. The two point velocity and concentration measurements document this observation. The plate and the flap were found to strongly influence the large scale structure of the jet.

Several conclusions can be reached. First, the flap serves to diminish the extent of influence of the large scale structures in the flow field. This should help in reducing the structural loads the flap or wing must endure. Instead of one dominant frequency in the velocity field, the turbulent fluctuations in the entire power spectrum would be amplified. The implication would then be to mount the 
nozzle as close as possible to the curved portion of the Flap. The second conclusion is in reference to comparing the velocity flow field with the two different velocity ratios, $\lambda_{j}$. There exists considerable doubt as to whether or not the jet flow will even remain attached as the velocity ratio between the inner jet flow and the tunnel flow tends towards infinity. The significance of measurements made in a static testing configuration $\left(\lambda_{j} \rightarrow \infty\right)$ is quite questionable. 


\section{APPENDIX A}

IDV Parameters and Velocity Field Properties

The IDV parameters include such items as the

characteristics of the data processing unit, the control volume, size and the signal-to-noise ratio of the Doppler bursts. Initially, consider the effects of the probe volume size. For the optical arrangement used in this investigation, the control volume was estimated to be the order of $0.05 \mathrm{~mm}$ (diameter) $\times 1 \mathrm{~mm}$ (Iength). (50)

Mean Velocity Analysis for Turbulent Flow

The mean velocity, J̈am, determined by a finite sized probe includes a space as well as a time averaging. Consider the following expression:

$$
\overline{\mathrm{J}} a \mathrm{~m}=\frac{1}{\mathrm{~A}} \iint\left[(1 / \mathrm{T}) \int_{0}^{\mathrm{T}} U(\mathrm{~A}, t) d t\right] d A
$$

where $U(A, t)$ is the instantaneous streamwise velocity, $t$ is time and $T$ is time interval for measurement.

For simplicity, assume a rectangular probe volume and a two dimensional flow field then

$$
\overrightarrow{\mathrm{U} a m}=(1 / \mathrm{w}) \int_{\mathrm{W} / 2}^{\mathrm{w} / 2}\left[(1 / \mathrm{T}) \int_{0}^{T} U(y, t) d t\right] d y
$$

where $w$ is probe volume dimension in the direction of the mean. velocity gradient, $y$, and $y$ equals $y-y_{0}$. 
Next, let $U(A, t)$ vary linearly with $y$ across the probe volume or

$$
\begin{aligned}
\quad U(A, t) & =U_{C}(t)+S y \\
\text { Then } \quad \bar{U}(A, t) & =\bar{U}(A)=\bar{U}_{C}+S y
\end{aligned}
$$

Substituting back into (2)

$$
\bar{U} a m=(1 / w) \int_{W / 2}^{W / 2}\left(\bar{U}_{0}+S y\right) d y
$$

Thus

$$
\overline{\mathrm{U}} \mathrm{am}=\overline{\mathrm{U}}_{\mathrm{C}}
$$

The conclusion being that the summation over a finite volume and time yields a good estimate of the mean velocity at the center of the probe when the average fluid velocity is linear.

\section{Root Mean Square Velocity Measurement}

The spatial and temporally averaged mean-square fluctuation, $\left(u_{m}\right)^{2}$ of the streamwise fluid velocity flowing through the probe volume is given by

$$
\left.\left(u_{m}\right)^{2}=(I / A) \iint\left[(I / T) \int\right]_{0}^{T}\left(U(A, t)-\bar{U}_{C}\right)^{2} d t\right] d A
$$

when the velocity fluctuations are referenced to the. time average velocity at the center of the probe volume. If we again assume that $U(A, t)=U(y, t)$, a function of $y$ alone and also that the volume is rectangular then

$$
\left(u_{m}\right)^{2}=(1 / w) \int_{-w / 2}^{W / 2}\left[(1 / T) \int_{0}^{T}\left(U(y, t)-\bar{U}_{C}\right)^{2} d t\right] d y
$$


Let us next assume that the velocity fluctuations are inversely proportional to the mean velocity. This makes sense as an approximation in the mixing region of the jet.

$$
\text { Then } \quad \dot{u}^{\prime}(y)^{\prime}=R / \bar{U}(y)=k /\left(\bar{U}_{C}+S y\right)
$$

Therefore

$$
\begin{aligned}
& u^{2}(\dot{y})^{2}=(t / T) s_{0}^{T}[U(y, t)-\bar{U}(y)]^{2} d t \\
& u^{\prime}(y)^{2}=k^{2} /\left(\bar{U}_{C}+S y\right)^{2}
\end{aligned}
$$

Rearranging (6)

$$
\begin{aligned}
u_{\mathrm{m}}{ }^{2}= & (1 / \mathrm{w}) \int_{\mathrm{w} / 2}^{\mathrm{W} / 2}\left[(1 / T) \int_{0}^{T}(U(y, t)-\bar{U}(y))^{2} d t\right. \\
& \left.+\left\{\bar{U}(y)-\bar{U}_{C}\right\}^{2}\right] d y
\end{aligned}
$$

Using (7) and (9), (10) may be integrated to yield

$$
u_{m}^{2}=\frac{S^{2} w^{2}}{I 2}+\frac{4 K^{2}}{4 \bar{U}_{C}^{2}-S^{2} w^{2}} .
$$

But $\mathrm{K}^{2} / \mathrm{U}_{\mathrm{C}}^{2}$ is the mean square fluctuation at $\mathrm{y}=0$ and therefore

$$
\left(u_{m}\right)^{2}=\frac{S^{2} w^{2}}{12}+\frac{4 K^{2} \cdot\left(u_{0}^{\prime}\right)^{2}}{4 K^{2}-S^{2} w^{2}\left(u_{0}^{\prime} \cdot\right)^{2}}
$$

The first term on the right hand side of (12) represents the "false turbulence" seen by the IDV due to a mean velocity.gradient across the control volume. The 
second term is a combination of the actual turbulent velocity variance and "false turbulence" due to change in mean square fluctuations across the probe volume.

For the experimental investigation which has been reported here, let us make an estimate of the magnitude of the "false turbulence" measured by the IDV, under the given assumptions.

Typical values of the parameters would be the following:

$$
\begin{aligned}
& \frac{u_{O^{\prime}}}{U_{0}}=0.1 \quad \mathrm{w}=1 \mathrm{~mm} \quad \mathrm{~K}=1 \times 10^{2} \mathrm{~m}^{2} / \mathrm{sec}^{2} \\
& U_{0}=10 \mathrm{~m} / \mathrm{sec} \quad \mathrm{S}=1 \times 10^{3} \mathrm{~m} / \mathrm{sec} / \mathrm{m} .
\end{aligned}
$$

Then, the actual variance of the flow field is:

$$
\left(u_{0}\right)^{2}=1 \mathrm{~m}^{2} / \sec ^{2}
$$

The measured velocity variance is:

$$
\begin{aligned}
\left(u_{\mathrm{m}}{ }^{\prime \prime}\right)^{2}= & {\left[\frac{\left(\frac{\left.1 \times 10^{3}\right)^{2}\left(1 \times 10^{-3}\right)^{2}}{12}+\right.}{}\right.} \\
& \frac{4\left(10^{4}\right)(1)}{\left.4\left(10^{4}\right)-\left(3 \times 10^{3}\right)\left(1 \times 10^{-3}\right)\right]} \mathrm{m}^{2} / \mathrm{sec}^{2} \\
\left(u_{\mathrm{m}}\right)^{2}= & .083+1.00 \mathrm{~m}^{2} / \mathrm{sec}^{2} \\
\left(u_{\mathrm{m}}\right)^{2}= & 1.083 \mathrm{~m}^{2} / \mathrm{sec}^{2}
\end{aligned}
$$


From the very simplified analysis, there are several conclusions that can be made. First, both the size of the control volume and the magnitude of the mean velocity gradient can have a non-negligible effect on the LDV turbulent intensity measurements. If at all possible, the smaller dimension of the control volume (length or height) should be aligned parallel to the direction of the largest mean velocity gradient.

Secondly, for the assumed form of u'(y), the velocity variance caused by the change in the mean square fluctuations across the probe volume is negligible. If a linear relationship between the mean velocity and fluctuating velocity were proposed (i.e. $u^{\prime}(y)=K \bar{U}(y)$ ) taken the contribution to the total variance would take the following form:

$$
\frac{s^{2} w^{2} A^{2}}{12}
$$

where $S$, w would take on the same value as before but $\mathrm{K}=1 \times 10^{-2}$.

Different functional forms for $u^{\prime}(y)$ where assumed and the results are shown in Table 3 .

Next, the characteristics of the signal processing unit (in this case a phase-locked loop) are also of paramount performance in the determining the accuracy of 


\section{TABLE 3}

Evaluation of Effect of Control Volume Size on Mean Square Fluctuations

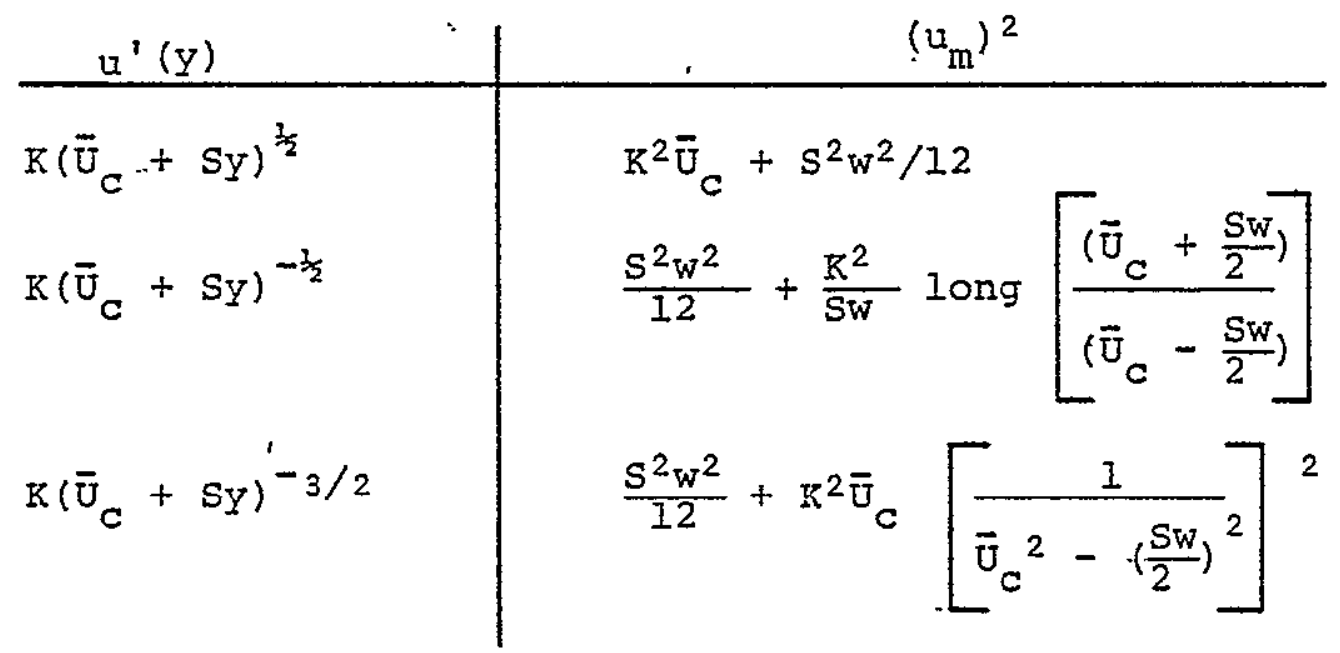


the IDV measurements. The phase locked-loop used is able to respond at a $14 \mathrm{kHz}$ rate to deviations from the centered or mean frequency (velocity) up to $\pm 28 \%$.

The unprocessed Doppler signal was fed into a Biomation Transient Recorder Model 8100. The Transient Recorder was used as it allows different segments of the Dopplex signal to be captured and displayed on an . oscilloscope. From this visual display, the frequency of the individual Doppler bursts can be determined, and, hence, if enough samples are displayed, a histogram can be constructed. Two histograms were obtained, one in the potentional core region of the freely expanding jet flow and the other determined in the shearing region of the velocity field: For the flow in the potential core region, the $\pm 28 \%$ range was more than adequate to handle the small turbulent fluctuations. In the shearing regions, though the turbulence was quite high (i.e. $\left.\left(\bar{u}^{2}\right)^{\frac{1}{2}} / U_{0}>0.1\right)$, the phase locked loop's range still. proved to be quite adequate. Out of the approximately 500 instantaneous frequencies measured, only about 5\% exceeded the limit of $\pm 28 \%$ of centered frequency. The signal-to-noise ratios for the Doppler signal were determined in a variety of ways. The first signal to noise ratios determined were for the signal before it was fed into the phase locked-loop. The filtered 
and amplified Doppler signal was analyzed by use of a Nelson Ross Spectrum Analyzer. The analyzer displayed the frequency content of the incoming signal. Here, two approaches were taken. First, with the Doppler signal present, an estimate of the signal to noise ratio could be determined from the visual display of the analyzer an oscilloscope. This ratio varied from about 100 to 1 , for the photodiode in a dual beam forward scattering mode, 75 to $I$ for the reference beam mode (diode), to approximately 20 to 1 for the photomultiplier tube in a back scattering location. Another estimate was made with the level of the Doppler signal compared to the noise present when one of the crossing laser beams is blocked. For the photomultiplier tube, the d.c. current was increased so as to be comparable to that present when the beams intersect in the test section. The signal to noise ratios determined from this procedure varied from about 100 to 1 for the dual beam mode (diode), 75 to 1 for the reference beam mode (diode), to about 15 to 1 for the photomultiplier tube in a back scattering mode. Since only the zero crossings are of interest, it would seem that the presence of the Doppler signal seems to override the noise in the case of the photomultiplier tube. The noise is thus superimposed on the Doppler signal. 
The third approach taken to determine the signal to noise ratios for the IDV setup involved using the output voltage, E, of the phase locked-1oop. Here the Erms of the Doppler signal was compared to the Erms output of the phaselocked-loop when, again, one of the crossing laser beams was blocked. The ratios determined in this manner ranged from 50 to 1 for the forward scattering dual beam mode (diode), 20 to I for reference beam mode. (diode), to about $15^{\circ}$ to 1 for the back scattering mode using the photomultiplier tube.

With the wide variance of signal-to-noise ratios for the forward scattering modes as compared to the back scattering mode, a logical step would be to compare the data obtained from the two setups. A sample of the results of this comparison is shown in rable 4.

In the sample data presented in Table 4 as well as for the remaining comparisons made the mean frequencies measured by all three modes of operation are extremely close. The rms voltages (i.e. turbulent intensity, $\left(\bar{u}^{2}\right) \frac{1}{2} /$ Uo) are nearly identical for the two forward scattering modes. Erms obtained from the photomultiplier tube used in a back scattering configuration is slightly less, though still reasonably close. This trend was consistent for all the comparisons that were made, both in the relatively quiet potential core part of the flow 


\section{TABIE 4}

$\begin{array}{ll}\text { Case 1 } & \frac{\text { Mode }}{} \\ \begin{array}{cl}\text { Dotential } \\ \text { Core) }\end{array} & \text { Reference } \\ & \text { Back Scatter }\end{array}$

Mean Frequency (MHz) Erms (Volts)

2.246

.460

$2.243 \quad .465$

$2.247 \quad .435$

$\begin{array}{clcc}\text { Case 2 } & \text { Mode } & \text { Mean Frequency (MHz) } & \text { Erms (Volts) } \\ \begin{array}{c}\text { Dual } \\ \begin{array}{c}\text { (Shearing } \\ \text { Region) }\end{array}\end{array} & 1.356 & 1.08 . \\ & \text { Reference } & 1.358 & 1.05 \\ & \text { Back Scatter } & 1.343 & 0.99\end{array}$


as well as in the highly turbulent shearing region. Presumably, this slight reduction in the turbulence perceived by the LDV in the back scattering mode is due to the fact that light scattered in this direction is several orders of magnitude less than is the case for a forward scattering mode. Even with the higher potential gain possible, the photomultiplier tube is, with the added constraint of a low noise level, not capable of detecting all the particles that are passing through the control volume. In essence, the contribution of these particles is "lost" in the noise.

Along with this discussion of signal-to-noise ratios it may prove to be instructive to compare in a qualitative manner, as well, the Doppler signals observed by the three different modes of IDV operation. (Figure 64). These drawings were made by use of the Biomation Transient Recorder. The control volume was located in the fully developed turbulent region of the freely expanding coflowing jet. There are two distinctions that can be made. One, the Doppler "bursts" in the forward scattering modes are fairly uniform envelopes. With the back scattering mode, however, there is quite a bit more ampltitude modulation of the signal. The second distinction deals with a comparison of the "dead" time (i.e. no particles in the scattering volume). For the 


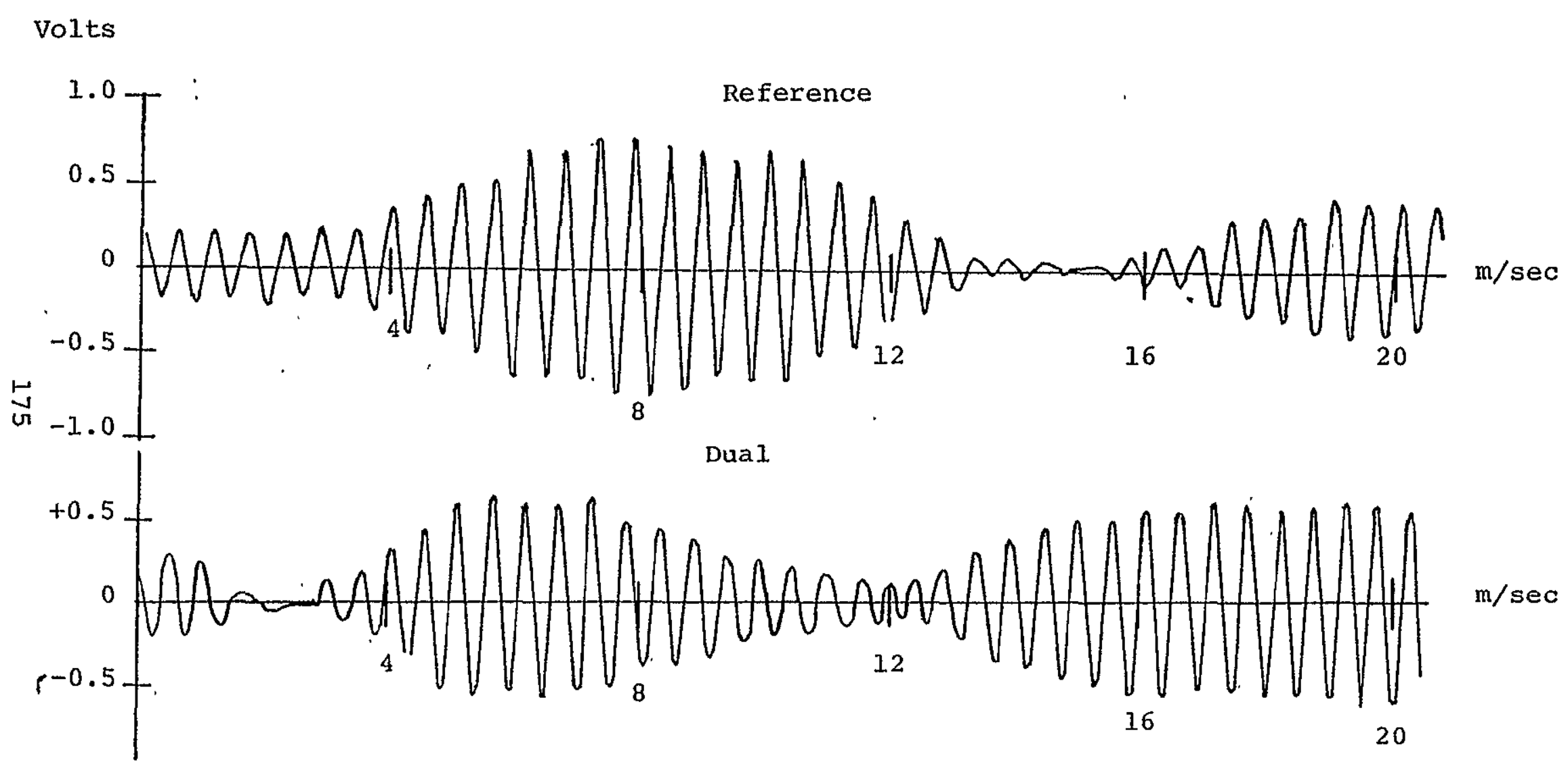

Figure 65(a) Doppler Signals in Forward Scattering Modes 


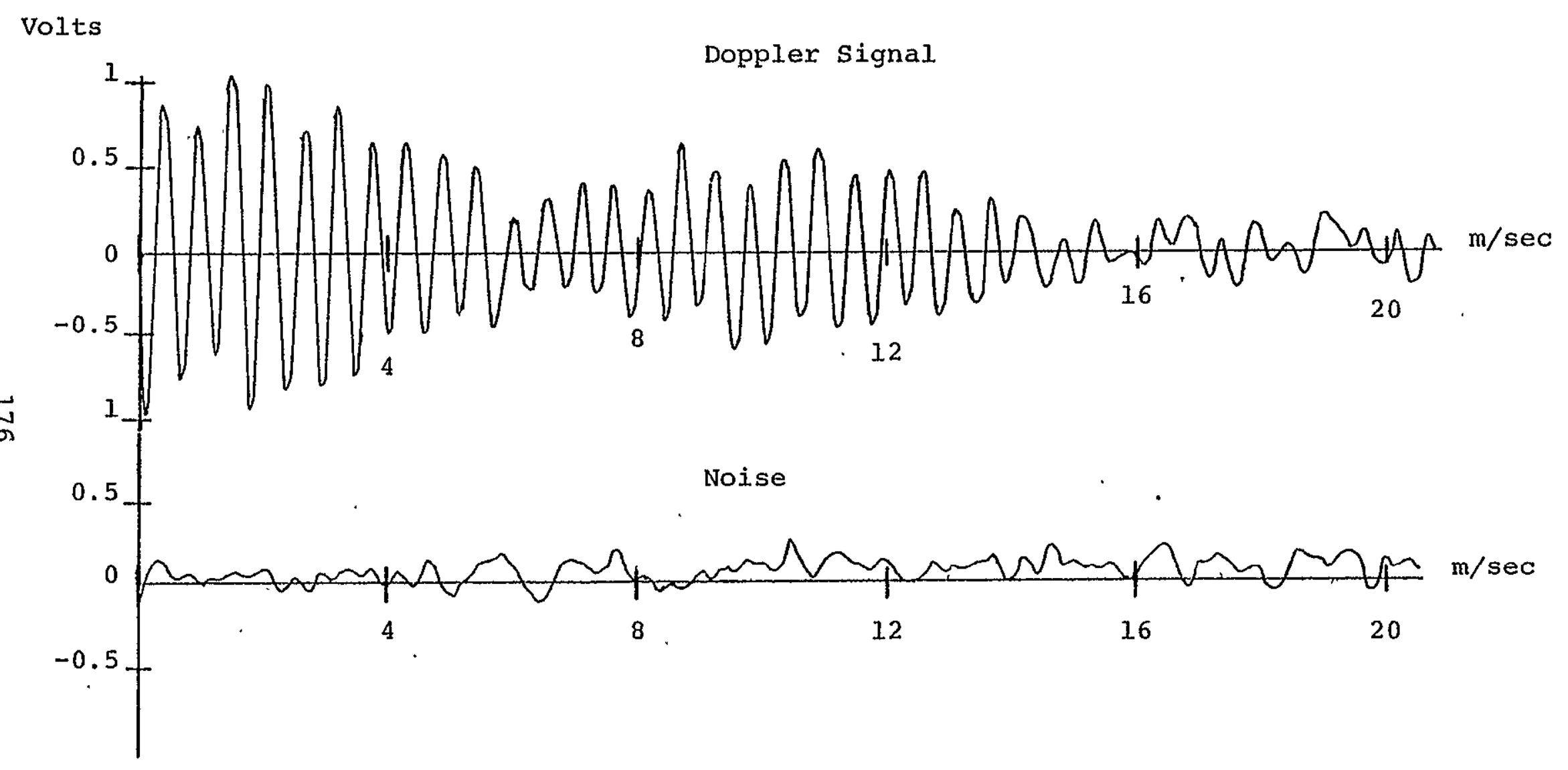

Figure 65(b) Dopplex Signal Versus Noise in Back Scattering Mode 
forward scattering modes, the "dead" time is distinct and easily distinguishable for the Doppler bursts. Such is not the case for the back scattering mode. It appears that some frequency component is virtually present the entire interval shown. The sharp distinction between signal and dead time is not longer present. 


\section{APPENDIX B}

\section{Particle Dynamics}

The IDV measures the particle velocities and the flow velocity is then inferred from this information. The question of how closely the particles follow the actual flow field is an extremely important one when considering the accuracy and reliability of the IDV.

Consider the equation of motion of a particle in a non-uniform flow under the assumption that the only effective force acting on the particle is the aerodynamic drag, and that the gas is much less dense than the particles (pg/pp $<$ I) then:

$$
\frac{d U p}{d t}=3 / 4 \cdot \frac{\mu}{\rho p D^{2}} R e_{p}: C_{D} \cdot\left(U_{g}-U_{p}\right)
$$

where $\mathrm{Ug}=$ velocity of gas

$$
\begin{aligned}
& U p=\text { velocity of particle } \\
& \operatorname{Re}_{p}=\frac{\rho g \cdot U_{g}-U_{p} \cdot D}{\mu}
\end{aligned}
$$

and $\quad C_{D}=f\left(R_{p}\right.$, Mach no., Knudsen no.)

Using stokes Law:

$$
C_{D}=\frac{24}{\operatorname{Re}_{p}}
$$


Inserting (2) in (1)

$$
\begin{aligned}
& \frac{d U p}{d t}=3 / 4 \frac{24}{\operatorname{Re}_{p}} \frac{\mu}{\rho_{p} D^{2}} \operatorname{Rep}\left(U_{g}-U_{p}\right) \\
& \frac{d U p}{d t}=\bar{k}(U g-U p) .
\end{aligned}
$$

where $\vec{K}$, the stokes coefficient is given by $\bar{k}=\frac{18 M}{\rho_{p} D^{2}}$ For this investigation,

$$
\begin{aligned}
& D=.3 \mu \mathrm{m}=.3 \times 10^{-6} \mathrm{~m} \\
& \text { uair }=1.796 \times 10^{-5} \mathrm{~kg} / \mathrm{m} \mathrm{sec} \\
& \sigma g=1.4 \text { or } \rho p=1.4 \times 10^{3} \mathrm{~kg} / \mathrm{m} 3 \\
& \text { Then: } \quad \overline{\mathrm{K}}=\frac{18\left(1.796 \times 10^{-5} \mathrm{~kg} / \mathrm{m} \mathrm{sec}\right)}{1.4 \times 10^{3} \mathrm{~kg} / \mathrm{m}^{3}\left(.3 \times 10^{-6} \mathrm{~m}\right)^{2}}=2.57 \times 10^{6}
\end{aligned}
$$

For an estimate of the particle lag, uㅡ, where $\underline{u}=$ Ug-Up, consider the flow in the nozzle of the jet. Assuming the flow velocity increases from about 0 to 15 $\mathrm{m} / \mathrm{s}$ within $.15 \mathrm{~m}$ then $\mathrm{Ug}$ may be approximated as a linear function along the nozzle, or.

$$
U g=\propto x \quad \text { where } \quad \propto=100
$$

Returning to (4) and making the approximations of a steady one dimensional flow with $\underline{u} \ll U \mathrm{Ug}$ then 


$$
U g \frac{d U g}{d x}=U g \frac{\dot{d} \underline{\underline{u}}}{d x}+\bar{k} \underline{\underline{u}}
$$

Substituting (5) into (6)

$$
\alpha^{2} x=\dot{c} x \frac{d \underline{u}}{d x}+\bar{K} \underline{\underline{u}}
$$

This can be integrated, and assuming no initial lag at. $\mathrm{x}=0$, then

$$
\underline{\underline{u}}=\frac{\alpha^{2}}{\alpha+\overline{\bar{k}}} x=\frac{\alpha}{\alpha+\overline{\mathrm{k}}} \mathrm{Ug}
$$

Thus at the exit plane of the nozzle, the lag can be estimated as:

$$
\underline{u}=\frac{1 \times 10^{2} \mathrm{Ug}}{\left(1 \times 10^{2}\right)+\left(2.57 \times 10^{6}\right)} \tilde{=} 4 \times 10^{-6} \mathrm{Ug}
$$

The particle lag is thus shown to depend on the velocity and viscosity of the flow, and the density and diameter of the particles. In this investigation, the lag is quite negligible.

Next consider a simple turbulent flow whose correlation function is assumed to be

$$
R(t)=\frac{\overline{\underline{u(t): u(t+t)}}}{\overline{\bar{u}^{2}}}=\exp \left(-2 \pi f_{t} \cdot t\right)
$$


where $f_{t}$ is a characteristic frequency of turbulence. The ratio of the rms particle velocity fluctuation, $\left(i_{p}{ }^{2}\right)^{\frac{1}{2}}$ to the rms fluid velocity fluctuation $\left(\bar{u}^{2}\right)^{\frac{1}{2}}$ is

$$
\frac{\left(\bar{u} p^{2}\right)^{\frac{1}{2}}}{\left(\bar{u}^{2}\right)^{\frac{3}{2}}}=\frac{1}{1+f t / f p} \quad \text { with } \quad f p=\frac{\bar{k}}{2 \pi}
$$

Recalling

$$
\text { fp }=\frac{2.57 \times 10^{6}}{2 \pi}=4 \times 10^{5} \mathrm{~Hz}
$$

Hence, the ratio $\frac{\left(\vec{u}^{2}\right)^{\frac{1}{2}}}{\left(\vec{u}^{2}\right)^{\frac{1}{2}}}$ is a function of the characteristic

frequency of the turbulence but for large $\overrightarrow{\mathrm{K}}$, the quantity tends toward 1.00 .

Essentially, the response of the particles act similar to a low pass filter. The $3 d B$ point of the rms response occurs at approximately $4 \times 10^{5} \mathrm{~Hz}$. 


\section{APPENDIX C}

\section{Phase locked loop processor}

\section{Description}

The basic function of the phase locked loop is to detect and track small differences in the frequency and phase between an input signal and a secondary reference signal. A simple diagram for the phase locked loop is shown in Figure $66(a)$. The PLI is a negative feedback loop consisting of a phase detector (PD), a low pass filter (IPF) and a controlled oscillator whose frequency is either a linear function of a dc voltage or current. The PII in this investigation used a current controlled oscillator (CCO).

As the loop tracks the incoming signal, the phase detector compares the frequency of the input to the output of the CCO. A change in the phase of the input is detected by the PD which produces an output proportional to the sum and difference in frequency and/or phase between the input signal and the cco output. The low pass filter then eliminates the sum component of the PD's output. An error signal is produced by the IPF whose magnitude is directly proportional to the phase difference determined at the phase detector. The error signal is then used to adjust the Erequency and phase of the cco in 


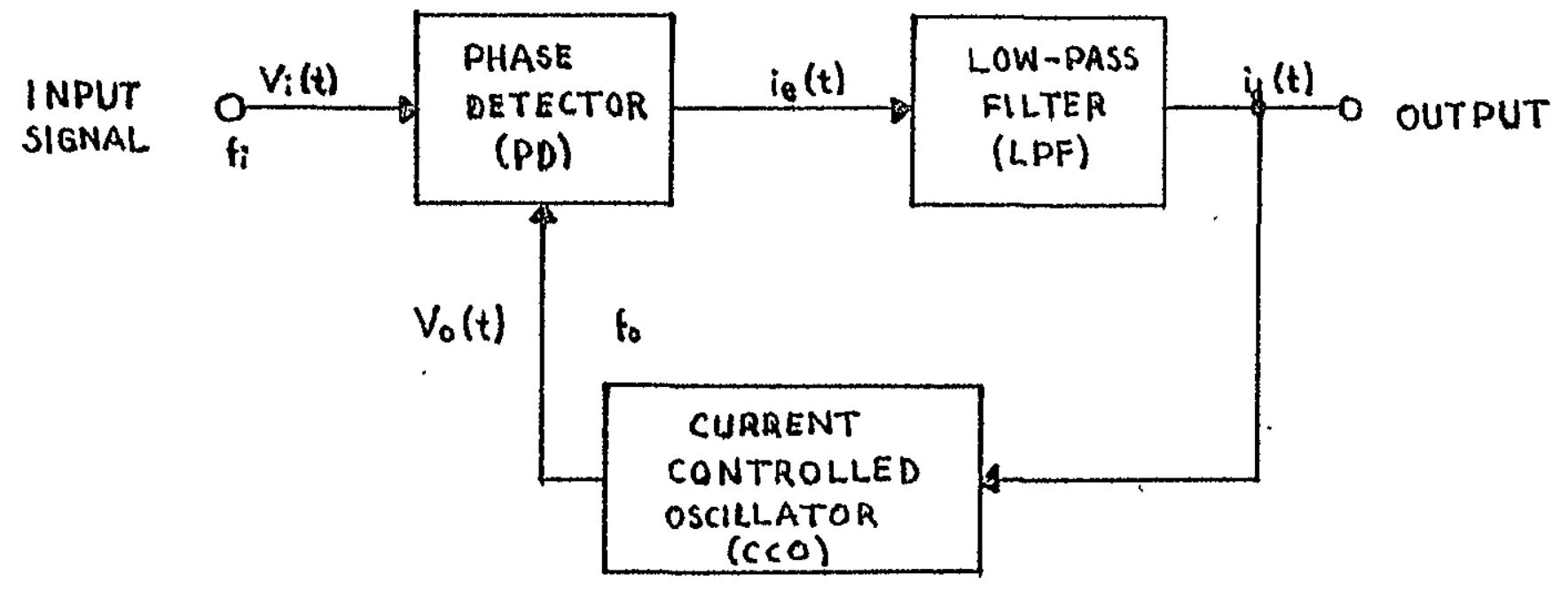

Figure 66(a) Feedback Loop Model

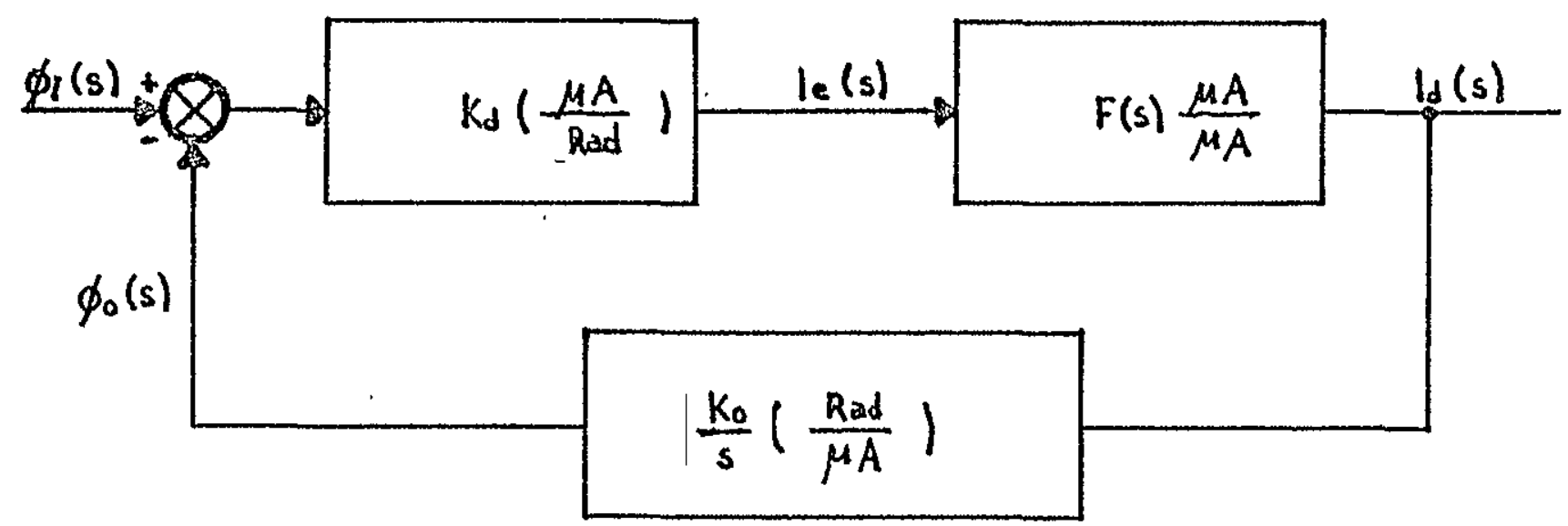

Figure 66(b) Linear Model of Phase Locked-Loop 
such a direction as to establish phase synchronization between the two signals at the phase detector.

Consider the following linear model for a PLI system, as shown in Figure $66(\mathrm{~b})$.

$\phi i$ and $\phi o$ represent phase angles associated with the input and CCO signals. $F(S)$ represents current transfer function of the IPF and $k_{d}$ and $k_{0}$ are conversior gains of the PD and CCO. Consider the input signal, Vi ( $t$ ) and the CCO output, Vo $(t)$ at the PD:

$$
\begin{aligned}
& v_{i}(t)=v_{i} \sin w_{i} t \\
& v_{0}(t)=v_{0} \sin \left(w_{0} t+\phi_{.}\right)
\end{aligned}
$$

where $w_{i}, w_{0}$ and $\phi$ are the frequency and phase characteristics of interest.

The PD's output current signal is:

$$
i_{e}(t)=k_{1} v_{i} v_{0}\left(\sin w_{i} t\right)\left(\sin \left(w_{0} t+\phi\right)\right)
$$

where $R_{1}$ is a constant. Case I:

$$
\begin{aligned}
& w_{i}=w_{0}=w \\
& i_{e}(t)=k_{1} v_{i} v_{0}(\sin w t)(\sin w t+\phi)
\end{aligned}
$$

Recalling $\sin a \sin b=\frac{1}{2} \cos (a-b)-\frac{1}{2} \cos (a+b)$ 
Then:

$$
i_{e}(t)=\frac{K_{1} V_{i} V_{o}}{2}(\cos \phi-\cos (2 \underline{w} t+\phi))
$$

The low pass filter removes the high frequency part and so:

$$
i_{e}(t)=i d=\frac{k_{2} V_{i} V_{0}}{2} \cos \phi
$$

where $\mathrm{K}_{2}$ is another constant.

If $w_{i}$ and $w_{0}$ are perfectly synchronized to the free running frequency $w_{i}$ then $i_{d}$ will equal zero which implies $Q$ must be $90^{\circ}$. If $w_{j}$ and $w_{0}$ are slightly out of phase then $i_{d}$ is proportional to the phase difference centered about the reference angle of $90^{\circ}$. If $w_{i}$ now changes from $w_{0}$ then $i_{d}$ will, adjust to a non-zero value in order to correct $w_{0}$ so that frequency lock can be maintained with $w_{i}=w_{0}{ }^{*} \phi$ will be shifted some amount $\triangle \phi$ from $90^{\circ}:$

Then $i_{\mathrm{d}}$ becomes

$$
i_{\mathrm{d}}=\mathrm{k}_{-2} \mathrm{ViVO} \cos (\phi \circ \pm \Delta \phi)= \pm \mathrm{K}_{2} \mathrm{~V}_{i} \mathrm{~V}_{0} \sin \Delta_{\phi}
$$

In addition to being an exror signal, $i_{d}$ represents the demodulated output of an FM input applied as $V_{i}(t)$. Case II: $w_{i} \frac{1}{T} w_{0}$

This case corresponds to an unlocked conditon for 
the loop. Hence the phase angle is meaningless and can be eliminated by appropriately choosing the time origin. The PD'S output is then:

$$
\left.i_{e}(t)=\frac{v_{i} v_{0} K \bar{j}}{2} \cdot\left(\cos \left(w_{i}-w_{0}\right) t-\cos \cdot w_{i}+\dot{w}_{0}\right) \cdot t\right) \cdot
$$

After filtering:

$$
i_{e}(t)=i_{d}(t)=k_{3} v_{i} v_{0} \cos \left(w_{i}-w_{0}\right) t
$$

where $\mathrm{K}_{3}$ is a constant.

Notice that $i_{d}(t)$ represents the signal current which is supplied to the CCO. If the amptitude of $V_{1}$ is sufficiently large and $w_{i}$ does not exceed the dynamic range of the cco then lock-in can be established. That is, the CCO's output frequency will be shifted from the free running frequency, $w_{0}^{\prime}$ by some $\Delta \dot{w}$. If $w_{i}$ is beyond the dynamic range, then $w_{0}$ should be shifted closer to the expected $w_{i}$ frequency.

\section{Specifications}

The PLI used in this investigation had the capability of determining the mean frequency (velocity) in the flow from $600 \mathrm{kHz}(3 \mathrm{~m} / \mathrm{sec})$ to $4 \mathrm{MHz}(20 \mathrm{~m} / \mathrm{sec})$. The useful frequency range over which the PII can track the input signal (i.e. Lock Range) is $\pm 36 \%$ of the centered frequency. The frequency range over which the PLI can 
acquire an input signal after lock has been lost (i.e. Capture Range) is set by the low pass filter's characteristics and has a value of $\pm 28 \%$ of the mean or centered Erequency.

For the calibration curves of the PLI and the techniques used for the actual acquisition of information, please see Reference 53. 


\section{APPENDIX D}

Determination of Transverse Velocity Data

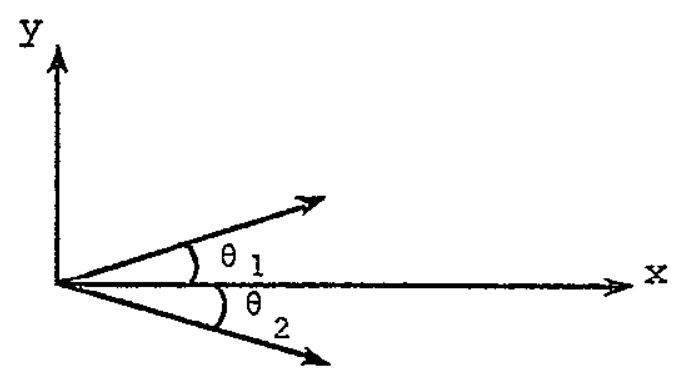

Since it is extremely difficult to directly measure the velocity in a airection perpendicular to the direction of the mean flow, the following scheme was devised.

Step 1: Measure velocity at a positive (+) and negative (-) angle with respect to the velocity $U$ at a point in the flow field. For simplicity, $\theta_{1}=-\theta_{2}=45^{\circ}$

Step 2:- For mean velocity:

$$
\begin{aligned}
& U+=U \cos \theta_{1}+V \sin _{1}=U \frac{\sqrt{2}}{2}+V \frac{\sqrt{2}}{2} \\
& U-=U \cos \theta_{2}+V \sin _{2}=U \frac{\sqrt{2}}{2}-V \frac{\sqrt{2}}{2}
\end{aligned}
$$

Then

$$
U=\frac{(U+)+(U-)}{\sqrt{2}}
$$


and

$$
\mathrm{V}=\frac{(\mathrm{U}+)-(\mathrm{U}-)}{\sqrt{2}}
$$

Step 3: For turbulent instensity:

$$
\begin{aligned}
& \overline{u_{+} 2}=\overline{\left(u \frac{\sqrt{2}}{2}+v \frac{\sqrt{2}}{2}\right)^{2}} \\
& u_{-2}=\overline{\left(u \frac{\sqrt{2}}{2}-v \frac{\sqrt{2}}{2}\right)^{2}}
\end{aligned}
$$

then

$$
\begin{aligned}
& \overline{u+2}=\frac{1}{2}\left(\bar{u}^{2}+2 \overline{u v}+\bar{v}^{2}\right) \\
& \overline{u-2}=\frac{1}{2}\left(\overline{u^{2}}-2 \overline{u v}+\overline{v^{2}}\right)
\end{aligned}
$$

and

$$
\left.\overline{v^{2}}=\overline{(u+2}+\overline{u-2}\right)-\overline{u^{2}}
$$




\section{REFERENCES}

1. Monin, A. S. and Yaglom, A. M. Statistical Fluid Mechanics Vol. 1, MIT Press, $197 \overline{1}$.

2. Roshko, A., "Structure of Turbulent Shear Flows: A New Look", AIAA Journal 'Vol. 14, No. 10, October 1976 .

3. Laufer, J., "Trends in Experimerital Turbulence Research", Annual Review of Fluid Mechanics, Vol. $7,1975$.

4. Townsend, A. A., The Structure of Turbulent Shear Flows, Cambridge University Press, 1956.

5. Grant, H. I., "The Iarge Eddies of Turbulent Motion" Journal of Fluid Mechanics, Vol. 4, 1958.

6. Kline, S. J., and Runstalder, P. W., Journal of Applied Mechanics, 26E: 166.

7. Brown, G. and Roshko, A., AGARD CPP-93 23:1.

8. Michalke, A., "On Spatially Growing Disturbances in an Inviscid Shear Iayer", Journal of Fluid Mechanics, Vol. 23, 1965.

9. Stuart, J. T. " Journal of Fluid Mechanics, Vol. 9

10. Wyganski, $I$. and Fiedler, H. "Some Measurements in the Self Preserving Jet", Journal of Fluid Mechanics, VOI. 38 $196 \overline{9}$.

II. Brown, G. I. and Roshko, A., "The Effect of Density Difference on the Turbulent Mixing Layer", Turbulent Shear Flows, AGARD-CP-93, 1971.

12. Brown, G. I., and Roshko, A., "On Density Effects and Iarge Structure in Turbulence Mixing Iayers", Journal of Fluid Mechanics, Vol. $64,1974$.

13. Winant, C., and Browand, F. K., "Vortex Pairing: The Mechanixm of Turbulent Mixing Layer Growth at Moderate Reynolds Number", Journal of Fluid Mechanics, Vol. 63, Pt. I, March, 1974. 
14. Moore, D. W., "A Numerical Study of the Rool-up of a Finite Vortex Sheet", Journal of Fluid Mechanics, Vol. 63, Pt. 2, 1974.

15. Davies, P.O.A.I., "Turbulence Structure in Free Shear Iayers", AIAA Journal, Vol. 4, Nov. 1966.

16. Crow, S. C., and Champagne, F. H., "Orderly Structure in Jet Turbulence", Journal of Fluid Mechanics, Vol. 48, Pt. 3, 1971 .

17. Yule, A. J., Bruun, H. H., and Baxter, D. R. J. and Davies P.O.A.I., Structure of Turbulent Jets, University of Southampton, ISVR Memo no. 506.

18. Rambe, T. and Takeo, T., "Mortion of Distorted Vortex Rings", Journal of Physical Society of Japan, Vo1. 31.

19. Oshima, Y., "Motion of Vortex Rings in Water", Journal of Physical Society of Japan, Vol. 32 .

20. Girard, J. P. and Curtet, R. M., "Time Evolution of Coherent structures in a Pulsating Jet", Colloquim on Coherent Structures in Turbulent, Southampton, 26-29, March 1974.

21. Binder, G. and Favre-Marinet, M., Unsteady Jets, I. F. R. F. Aerodynamic Panel, France, 1972.

22. Catalano, G. D., Morton, J. B., and Humphris, R. R., "Experimental Investigation of an Axisymmetric Jet in a Coflowing Airstream", AIAA Journal, Vol. 14, No. 9, September 1976 .

23. Glauert, M. B., "The Wall Jet", Journal of Fluid Mechanics, Vol. 1, 1956.

24. Forthman, E., "Turbulent Jet Expansion", NACA TM 789, March, 1936.

25. Schwarz, W. H., and Cosart, W. P., "The Two Dimensional Turbulent Wall Jet", Journal of Fluid Mechanics, Vol. 10, Pt. 4, 1961.

26. Sigalla, A., "Measurements of Skin Friction in a Plane Turbulent Jet", The Aeronautical Journal of the Royal Aeronautical Society, England, Vol. 62, 1958 . 
27. Myer, G. E. Schauer, J. J., and Eustis, R. H., "The Plane Turbulent Wall Jet, Pt. 1, Jet Development and Friction Factor", Tech. Report 1, Mech. Engr. Dept., Stanford University, Stanford, Ca., June, 1961.

28. Hari, V., "A Method to Analyze the Wall Jet Flow", Aeronautical Journal, October, 1973.

29. Dvorak, F. A., "Calculation of Turbulent Boundary Iayers and Wall Jets over Curved Surfaces", AIAA Journal, Vol. 11, No. 4, April 1973.

30. Blackwelder, R. F., and Kovasznay, L., "Time Scales and Correlations in a Turbulent Boundary Layer", The Physics of Fluids, Vol. 15, No. 9, Sept. 1972.

31. Rajaratnam, N., and Paris, B. S., "Three Dimensional Turbulent Wall Jets", Journal of Hydraulics Division, IASME Transactions., January 1974.

32. Narain, J. P., "Three Dimensional Turbulent Wall Jets", The Canadian Journal of Chemical Engineering, Vol. 53, June 1975 .

33. Hill, P. G., "Turbulent Jets in Ducted Streams", Journal of Eluid Mechanics, Vol. 22, Pt. 1, 1965.

34. Catalano, G. D., "Experimental Investigation of a Coflowing Jet", M. S. Thesis, University of Virginia, May 1975 .

35. Iawrence, J. C., NACA Report 1292, 1955.

36. Sarni, S., Carmody, T., and Rouse, H., "Jet Diffífion in the Region of Flow Establishment", Journal of Fluid Mechanics, Vol. 29, July, 1967.

37. Ko, N. W. M. and Davies, P. O. A. I., "The Near Field Within the Potential Core of Subsonic Cold Jets", Journal of Fluid Mechanics, Vol. 50, November 1971.

38. Bradshaw, P., Fevis, D. H. and Johnson, R. J., "Turbulence in Noise Producing Region of Circular Jets", Journal of Fluid Mechanics, Vo. 19, August 1964 .

39. Korbacher, G. K., "Aerodynamics of Powered HighIift Systems", Annual Review of Fluid Mechanics, Vol. $6,1974$. 
40. Berricht-Rapport R117/76 Proceeding of the ISL/Agard Workshop on Iaser Anemonetry, May 5-7, 1976, SainteLouis, France.

41. Proceeding of the Second International Workshop on Laser Velocimentry, Thompson, H. D. and Stevenson, W. H. Editors, Purdue University, March 27-29, 1974.

42. Kried, D. R., "Iaser Doppler Velocimeter Measurements in Non-Uniform Flow: Error Estimates" Applied Optics, Vo1. 13, No. 8, August, 1974.

43. Karpuk, M. E., and Tiederman, W. G., "Effect of Finite Size Probe Volume upon Laser Doppler Anemometer Measurements", AIAA Journal, Vol. 14, No. 8, August 1976.

44. Baker, R. J., Hutchman, P., and Whitelaw, J. H., "Preliminary Measurements of Instantaneous Velocity in a Two-Meter Square Furnace Using a Laser Anemometer, Transactions of ASME, Journal of Heat Transfer, August 1974 .

45. Wisler, D. C., and Mossey, P. W., "Gas Velocity Measurements Within a Compressor Rotor Passage Using the Laser Doppler Velocimeter", Transactions of ASME, Journal of Engineering for Power,-April 1973 .

46. Munoz, R. M. Mocker, H. W., and Koehler, Lyle, "Airborne Iaser Doppler Velocimeter", Applied Optics, Vol. 13, No. 12, December 1974.

47. Hughes, À. J., and Pike, E. R., "Remote Measurement of Wind Speed by Laser Doppler Systems", Applied Optics, Vo1. 12, No. 3, March 1973.

48. Hallermeier, R. J., "Design Considerations for a 3-D IDV for Studying Gravity Waves in Shallow Water", Applied Optics, Vol. 12, No. 2, February 1972 .

49. Owen, F. K., "Laser Velocimeter Measurements in Free and Confined Coaxial Jets with Recirculation", A Paper No. 75-120, AIAA 13th Aerospace Sciences Meeting, Pasadena, Calif., Jan. 20-22, 1975.

50. Wang, T. Clifford, S. F., and Ochs, G. R., "Wind and Refractive-Turbulence Sensing Using Crossed Laser Beams", Applied Optics, Vol. 13, No. 11, November 1974. 
51. Pfeiffer, H., vom Steim, H. D., and Koch, B., "Investigation of a Supersonic Free Jet by Means of a Laser Anemometer", Applied Physics, Vol. 4, 1974.

52. Yanta, W. J., and Lee, R. E., "Determination of Turbulence Transport Properties with the LDV and Conventional Time-Averaged Mean Flow Measurement at Mach 3", AIAA 7th Fluid and Plasma Dynamics Conference, Paper No. 74-575, June 1974.

53. Rose, W. Co. and Johnson, D. A., "A Study of Shock Wave Turbulent Boundary Layer Interaction using Laser Velocimeter and Hot-Wire Anemometer Techniques", AIAA Paper No. 74-95, AIAA Aerospace Science Meeting, Washington, D. C., Jan. 1974.

54. Flack, R. D., and Thompson, H. D., "Preliminary Results with purdue's LDV System", Purdue II, VoI. 1.

55. Clark, W. H., "Measurements of Two Point Veloci.ty Correlations in a Pipe Flow Using Iaser Aneometers", Ph.D. Thesis, The University of Virginia, 1970.

56. Haertig, J., "Introductory Iecture on Particle Behavior", Proceedings of the ISI/AGARD Workshop on' Laser Anemometry, German-French Research Institute, May 5-7; 1976 .

57. Schroeder, J. C., "Development of Experimental Techniques for Investigation of Unsteady Pressures Behind a Cold Model Jet", Master's Thesis, University of Virginia, May 1976.

58. Herling, W. W., "The Development of an Experimental Facility for the Investigation of Scaling Effects in Propulsive Iift "Configurations", Master's Thesis, University of Virginia, May 1976.

59. Shaughnessy, E. J., Jr., "Measurement of Particle Diffusion in a Turbulent Jet -by laser Iight Scattering", Ph.D. Dissertation, The University of Virginia, December 1975 .

60. Pabst, O'., Die Ausbreitung Neisser Gasstrahlen in Bewegter Luft II, Teil, UM 8007, 1944.

61. Morton, J. B., "Experimental Measurements of Ambiguity Noise in a Iaser Anemometer", Journal of Physics, E., Vol. 6, No. 4, April 1973. 
62. Ko, N. M. U., and Davies, P. O. A. I., "The Near Field within the Potential Core of Subsonic cold Jets", Journal of Fluid Mechanics, Vol. 50, Pt. I, 1971.

63. Van Atta, C. W., "Sampling Techniques in Turbulence Measurements", Annual Review of Fluid Mechanics; Vol. 6, 1974 .

64. Sternberg, J., "On the Interpetation of Space Time Correlation Measurements in Shear Flow", The

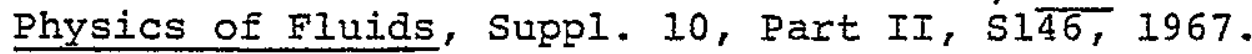

65. Favre, A., "Review on space Time Correlations in Turbulent Fluids", Journal of Applied Mechanics, Transactions of ASME, Vol. 32E, 241, 1965.

66. Favre, A., Gaviglio, J., and Dumas, R., "Structure of Velocity space Time Correlations in A Boundary Layer", The physics of Fluids, Suppl. 10, Part II, S138, 1967. 